\title{
Diagnostic modalities and outcome measures in upper gastrointestinal disorders
}

Citation for published version (APA):

Smeets, FGM. (2019). Diagnostic modalities and outcome measures in upper gastrointestinal disorders. [Doctoral Thesis, Maastricht University]. Maastricht University. https://doi.org/10.26481/dis.20190927fs

Document status and date:

Published: 01/01/2019

DOI:

$10.26481 /$ dis.20190927fs

Document Version:

Publisher's PDF, also known as Version of record

\section{Please check the document version of this publication:}

- A submitted manuscript is the version of the article upon submission and before peer-review. There can be important differences between the submitted version and the official published version of record.

People interested in the research are advised to contact the author for the final version of the publication, or visit the DOI to the publisher's website.

- The final author version and the galley proof are versions of the publication after peer review.

- The final published version features the final layout of the paper including the volume, issue and page numbers.

Link to publication

\footnotetext{
General rights rights.

- You may freely distribute the URL identifying the publication in the public portal. please follow below link for the End User Agreement:

www.umlib.nl/taverne-license

Take down policy

If you believe that this document breaches copyright please contact us at:

repository@maastrichtuniversity.nl

providing details and we will investigate your claim.
}

Copyright and moral rights for the publications made accessible in the public portal are retained by the authors and/or other copyright owners and it is a condition of accessing publications that users recognise and abide by the legal requirements associated with these

- Users may download and print one copy of any publication from the public portal for the purpose of private study or research.

- You may not further distribute the material or use it for any profit-making activity or commercial gain

If the publication is distributed under the terms of Article $25 \mathrm{fa}$ of the Dutch Copyright Act, indicated by the "Taverne" license above, 
Diagnostic modalities and outcome measures in upper gastrointestinal disorders 


\section{Colofon}

(C) Copyright Fabiënne Smeets, Maastricht, 2019.

All rights reserved. No part of this book may be reproduced or transmitted in any form or by any means, without prior permission in writing by the author, or when appropriate, by the publishers of the publications.

ISBN 978-94-92741-27-1

Cover/design: Daniëlle Smeets

Layout: Tiny Wouters

Production: Drukkerij Walters, Maastricht

The printing of this thesis was financially supported by Maastricht University, Maastricht Universitair Medisch Centrum ${ }^{+}\left(\mathrm{MUMC}^{+}\right)$, and 'Nederlandse Vereniging voor Gastroenterologie'. 


\title{
Diagnostic modalities and outcome measures in upper gastrointestinal disorders
}

\author{
PROEFSCHRIFT \\ ter verkrijging van de graad van doctor aan de Universiteit Maastricht, \\ op gezag van de Rector Magnificus, Prof. Dr. Rianne M. Letschert, \\ volgens het besluit van het College van Decanen, \\ in het openbaar te verdedigen \\ op vrijdag 27 september 2019 om 10.00 uur
}

door

Fabiënne Geraldine Maria Smeets 
Promotor

Prof. dr. A.A.M. Masclee

Co-promotoren

Dr. J.M. Conchillo

Dr. D. Keszthelyi

Beoordelingscommissie

Prof. dr. G.A. Van Koeveringe (voorzitter)

Dr. G.H. Koek

Prof. dr. B.P.F. Rutten

Prof. dr. A.J.P.M. Smout (Academisch Medisch Centrum Amsterdam)

Prof. dr. T. Vanuytsel (Katholieke Universiteit Leuven, België) 


\section{Contents}

$\begin{array}{lll}\text { Chapter } 1 \quad \text { General introduction } & 7\end{array}$

Part I Patient-reported outcome measures in functional dyspepsia $\quad 23$

Chapter 2 Systematic review: disease-specific instruments to assess 25

gastrointestinal symptoms in functional dyspepsia

Chapter 3 Development of a real-time patient-reported outcome measure

73 for symptom assessment in patients with functional dyspepsia using the experience sampling method

Part II Prediction of treatment outcome in upper gastrointestinal disorders

Chapter $4 \quad$ Esophagogastric junction distensibility in the management of achalasia patients: relation to treatment outcome

Chapter 5 Effect of transoral incisionless fundoplication on reflux mechanisms

Chapter 6 Does measurement of esophagogastric junction distensibility by EndoFLIP predict therapy-responsiveness to endoluminal fundoplication in patients with gastroesophageal reflux disease?

Chapter 7 Endoscopic botulinum toxin for gastroparesis:

results of a retrospective series

Chapter 8 General discussion

Addendum Summary

Samenvatting

Valorization

List of publications

Dankwoord

Curriculum vitae 

Chapter 1

General introduction 



\section{General introduction}

\section{Overview}

Upper gastrointestinal symptoms occur frequently among the general population, are disabling, and a reason for consultation in primary care. In 2016 the incidences of 'stomach pain' and 'heartburn' were 22/1000 and 7/1000 patients respectively. ${ }^{1,2}$ For first line treatment, it is not necessary to strictly distinguish between patients with these complaints as initial treatment is identical with acid suppressive medication. In case of persistent and/or recurrent symptoms, patients are often referred for an upper gastrointestinal endoscopy. Although it is assumed that signs of gastroesophageal reflux disease (GERD), ulcer disease and malignancy will be present in $20 \%, 5 \%$ and $<1 \%$ of patients respectively, no (visible) explanatory abnormalities will be found in the majority of patients (60-70\%). ${ }^{3}$ Patients in the latter group are often considered as patients with 'functional' instead of somatic complaints, though a subgroup might have a disturbed gastrointestinal motility (e.g. achalasia, gastroparesis).

Functional gastrointestinal disorders (FGIDs) are some of the most common digestive disorders affecting at least one-third of the general population. ${ }^{4}$ Almost half of the patients with gastrointestinal symptoms referred to gastroenterologists will ultimately be diagnosed with FGID and $19.2 \%$ will have more than one FGID, which comprises a variety of clinical entities including functional dyspepsia (FD), functional heartburn and irritable bowel syndrome (IBS) according to the Rome IV criteria. ${ }^{4-6}$

Despite the lack of an organic cause for functional gastrointestinal disorders (e.g. functional dyspepsia), they share several characteristics with somatic disorders (e.g. gastroesophageal reflux disease, achalasia, gastroparesis). First, pathophysiological mechanisms are still incompletely understood for both groups of disorders. Second, accurate evaluation of treatment outcome is an important methodological challenge especially in patients with functional gastrointestinal disorders as they are symptombased without available biological markers to monitor treatment efficacy. In the past years, increasing effort has been made to develop a valid set of biomarkers to predict treatment outcome in patients with IBS. Despite the availability of several faecal, blood, and radiographic parameters, currently no (set of) biomarker(s) is available as reliable diagnostic tool, therapeutic target or prognostic indicator. This is probably due to the multifactorial nature of the disorder. ${ }^{7}$ The underlying etiologic heterogeneity in functional dyspepsia explains that an 'one-size-fits all' approach to treatment yields lackluster results and warrants a shift from symptom-based strategies to mechanism-based approaches to identify biomarkers predictive of treatment response. ${ }^{8}$ Although identification of biomarkers is eagerly awaited, assessment of treatment efficacy is currently based on symptom registration by patients using patient-reported outcome measures (PROMs). 
Therefore, the first part of this thesis aims to describe the available PROMs for symptom assessment in patients with functional dyspepsia together with their psychometric validation properties. Moreover, the development of a novel PROM based on the Experience Sampling Method is described.

Besides the challenging evaluation of treatment efficacy, several treatment options have beneficial effects in selected subgroups of patients which might be due to the etiologic heterogeneity underlying upper gastrointestinal disorders. ${ }^{9-12}$ Adequate identification and selection of patients with potential beneficial treatment effect by the use of diagnostic modalities (e.g. manometry, endoluminal functional imaging probe (EndoFLIP)) seems warranted to optimize treatment, and reduce health care costs.

The second part of this thesis therefore aims to evaluate the use of diagnostic modalities for assessment of treatment efficacy and to predict treatment outcome in patients with upper gastrointestinal disorders treated with (endoscopic) minimally invasive procedures.

\section{Part I Patient-reported outcome measures in functional dyspepsia}

In the first part of this thesis, we focus on patient-reported outcome measures (PROMs) for symptom assessment in functional dyspepsia. This introduction will give a brief overview of functional dyspepsia, PROMs and the Experience Sampling Method.

\section{Functional dyspepsia}

Functional dyspepsia (FD) is one of the most common functional gastrointestinal disorders with an estimated prevalence of $11-29 \%$ in the general population. ${ }^{13}$ Functional dyspepsia is defined by the presence of bothersome upper gastrointestinal symptoms without evidence of an organic, structural or metabolic disease that is likely to explain these symptoms. ${ }^{5}$ According to their predominant symptoms, patients can be divided into (a) postprandial distress syndrome marked by the presence of bothersome postprandial fullness occurring after normal-sized meals and/or by early satiety which prevents finishing a regular-sized meal, or (b) epigastric pain syndrome which consists of epigastric pain and/or burning. ${ }^{5,14}$ Dyspeptic symptoms lead to impaired quality of life, reduced work productivity and increased health care costs. ${ }^{5,15,16}$ This economical and societal burden underlines the need for (development of) effective treatment options.

The latter is however hindered by the multifactorial and incompletely understood pathophysiology which makes treatment challenging. ${ }^{5,17,18}$ In the last decades, treatment changed from a 'diagnosis-based one-size-fits all' strategy to a 
'symptom-based' strategy by dividing patients into subtypes according to their predominant symptoms (i.e. postprandial distress syndrome and epigastric pain syndrome). ${ }^{8}$ This 'symptom-based' strategy was based on the hypothesis that different pathophysiological mechanisms would contribute to distinct predominant symptoms, and that targeting these more specific symptom clusters would enhance patient homogeneity and improve treatment outcome. However, varying results have been described with regard to differences in gastric emptying time, gastric accommodation, and visceral hypersensitivity between FD subtypes. ${ }^{19-21}$ In addition, contradictory results have been reported for symptom-based treatment, which might probably be due to the considerable subgroup of patients with overlapping EPS and PDS symptoms. ${ }^{22,23}$ Recently, a new management algorithm for functional dyspepsia has been published in the new American College of Gastroenterology (ACG) guideline which recommends the consecutive use of acid suppressive medication, antidepressants and prokinetic agents regardless of FD subtype. ${ }^{24}$

\section{Patient-Reported Outcome Measures (PROMs)}

Besides development of new treatment options, evaluation of treatment efficacy is a methodological challenge as functional dyspepsia is a symptom-based diagnosis and no objective outcome parameters or biological markers are currently available. ${ }^{5}$ Therefore, use of Patient-Reported Outcome Measures (PROMs) is recommended by regulatory authorities (e.g. the Food and Drug Administration (FDA)) to assess treatment outcome in patients with functional gastrointestinal disorders. A PROM is defined as 'any report of the status of a patient's health condition that comes directly from the patient, without interpretation of the patient's response by a clinician or anyone else'. ${ }^{25}$ Development of a PROM is a comprehensive process and consists of several steps: (a) development of a conceptual framework based on an extensive literature review, (b) modification of this conceptual framework by input from patient focus groups, (international) experts in the field, and cognitive interviews, and (c) evaluation of psychometric validation properties including reliability, validity and responsiveness. ${ }^{25}$

Despite the availability of multiple outcome measures, currently no PROM has been approved by regulatory authorities and use of this variety of retrospective questionnaires in clinical trials makes comparison between studies challenging. Moreover, several limitations of currently available outcome measures should be considered including recall bias, ecological bias and non-compliance which warrants development of a new reliable PROM. ${ }^{26,27}$

\section{Experience Sampling Method}

The lack of one universal accepted PROM together with several important limitations of retrospective questionnaires warrants the need for development and psychometric 
validation of a new PROM. Real-time assessment of dyspeptic symptoms to minimize recall and ecological bias, and use of an e-health approach to improve patient comfort and reduce non-compliance seems desirable for the future. ${ }^{27}$

The Experience Sampling Method (ESM), also referred to as Ecological Momentary Assessment (EMA), is an attractive digital method for real-time symptom assessment that may potentially meet with all of the above mentioned requirements. EMA aims 'to assess the ebb and flow of experience and behaviour over time, capturing life as it is lived, moment to moment, hour to hour, day to day, as a way of faithfully characterizing individuals and of capturing the dynamics of experience and behaviour over time and across settings' (sic). ${ }^{28,29}$

This definition comprises several key features of EMA approaches: (a) Random, repeated assessments are used for several consecutive days to assess current symptoms, which is considered the momentary aspect of EMA in order to reduce recall bias. ${ }^{28,30}$ (b) Data collection is performed in patients' real life which improves ecological validity. ${ }^{28,30}$ (c) Compliance is increased by use of digital data collection, as a high rate of fake compliance has been described by using paper end-of-day diaries. ${ }^{27}$ (d) Repeated symptom assessment provides information about symptom variability over time and across situations as it takes into account the influence of several contextual (e.g. company, location, activities, food or substance intake), and psychological factors (e.g. stress). (e) Acquisition of information about temporal sequences of symptoms provides insight into antecedents or consequences of events, behaviours or triggers. For functional gastrointestinal disorders this key feature of ESM is considered important, as multiple factors might contribute to symptom generation and/or exacerbation including meal intake (e.g. meal size, specific kinds of food), medication use (e.g. non-steroidal inflammatory drugs; NSAIDs) and psychological stress. ${ }^{30-34}$ Taking into account the high prevalence of psychiatric comorbidity in functional dyspepsia, the presence of psychological factors is likely to affect symptom reporting. ${ }^{35-37}$ Identification of these psychological and contextual factors with influence on gastrointestinal symptoms might lead to more effective and individualized treatment regimens. Despite these favorable features, use of the ESMprinciple in gastrointestinal disorders is currently limited to small trials in patients with IBS.

\section{Part II Prediction of treatment outcome in upper gastrointestinal disorders}

In the last decades, a shift has been observed from invasive surgical treatment options to minimally invasive (endoscopic) treatment modalities for upper gastrointestinal disorders to (a) reduce (per)operative risks (e.g. bleeding, perforation, mortality) and 
(b) prevent development of (long-term) complications (e.g. dysphagia after traditional antireflux surgery). ${ }^{9,10,38}$ However, these minimally invasive treatment options appear to be effective for a subgroup of patients only. ${ }^{9-12}$ Use of diagnostic modalities to adequately select patients for these minimally invasive interventions seems therefore warranted in order to improve treatment outcome and reduce health care costs.

In the second part of this thesis, we therefore focus on the use of diagnostic modalities to predict treatment outcome in patients with several upper gastrointestinal disorders treated with (endoscopic) minimally invasive interventions.

\section{Achalasia}

Achalasia is a primary esophageal motility disorder characterized manometrically by insufficient relaxation of the lower esophageal sphincter (LES) and loss of esophageal peristalsis. ${ }^{39}$ The classic presentation of achalasia consists of dysphagia to solids and liquids with associated regurgitation of bland, undigested food or saliva. Additional symptoms are weight loss, substernal chest pain and heartburn. ${ }^{40}$ Although the definite cause of achalasia remains unknown, three factors seem to be involved: (a) genetic predisposition, (b) environmental triggers (e.g. viruses) and (c) autoimmune response. ${ }^{41}$ Environmental factors might trigger an auto-immune response in genetic susceptible individuals leading to a spectrum of histopathological changes at different stages of achalasia. ${ }^{39,42}$ Early in the disease there is myenteric inflammation with ganglionitis but without ganglion cell loss or neural fibrosis. Progression of the disorder is associated with progressive destruction of inhibitory neurons and neural fibrosis, and might explain the existence of different manometric subtypes according to the Chicago classification. ${ }^{42}$ As inhibitory neurons are normally involved in LES and esophageal relaxations by the release of nitric oxide (NO) and vasoactive intestinal polypeptide (VIP), loss of these inhibitory neurons in achalasia patients leads to impaired LES relaxation and loss of esophageal peristalsis on manometric recording. ${ }^{42}$

Treatment options aim to reduce the hypertonicity of the LES by either pharmacologic, endoscopic or surgical modalities in order to relieve symptoms, improve esophageal emptying and prevent further esophageal dilation. ${ }^{39}$ Pharmacologic therapy with nitrates, calcium channel blockers and botulinum toxin injection have limited roles in treatment of achalasia and are often reserved for highrisk patients not eligible for more definitive therapies. ${ }^{43}$ Graded pneumatic balloon dilation or laparoscopic surgical myotomy with partial fundoplication are recommended as initial therapy. ${ }^{40} \mathrm{~A}$ large European multicenter randomized trial compared laparoscopic Heller myotomy (LHM) in combination with Dor's fundoplication with endoscopic pneumatic balloon dilation in newly diagnosed achalasia patients, and described comparable success rates between both treatment groups after 2 and 5 years follow-up (i.e. 2 years follow-up: 90\% versus 86\%; 5 years follow-up: $84 \%$ versus $82 \%$, LHM vs. pneumatic balloon dilation respectively). ${ }^{44,45}$ 
Moreover, basal LES pressure, esophageal emptying and quality of life were comparable although $25 \%$ of patients treated with pneumatic balloon dilation required redilation therapy. ${ }^{45}$ Peroral esophageal myotomy (POEM) is a more recently developed endoscopic technique for treatment of achalasia which appears to be safe though occurrence of gastroesophageal reflux is a major concern. Long-term efficacy of POEM is still unknown although a large single center series of 500 patients described a success rate of $91.3 \%$ after 2 months which decreased to $88.5 \%$ (in 105 patients) after 3 years follow-up. ${ }^{46}$ Deteriorating efficacy over time has also been described by Werner et al. with success rates of $93.7 \%$ at 3-6 months follow-up and 78.5\% after 24-49 months follow-up. ${ }^{47}$

Declining success rates over time after achalasia treatment underline the need for short-term follow-up to gauge treatment efficacy and the need for retreatment in order to reduce development of long-term complications (e.g. esophageal decompensation, esophageal dysplasia and neoplasia). ${ }^{40}$ Early recognition of patients in need for retreatment is however challenging due to underreporting of symptoms by esophageal decompensation or impaired sensitivity. Therefore, functional testing is preferred over symptom evaluation to assess treatment efficacy. ${ }^{40}$ Previous studies evaluated the role of postintervention timed barium esophagram and manometry to predict treatment outcome and described varying results. ${ }^{48-51}$ In a recent study, posttreatment LES pressure was not able to predict treatment outcome and therefore it was hypothesized that esophageal emptying and symptom generation might be dependent on other factors than LES pressure. ${ }^{51}$ One parameter of interest is the distensibility of the esophagogastric junction (EGJ) as flow across the EGJ is directly proportional to EGJ diameter to the fourth power. ${ }^{52}$ Previous studies used a combined manometric-barostat device to assess EGJ distensibility in patients with gastroesophageal reflux disease with and without hiatal hernia, and after fundoplication. ${ }^{52-54}$ This technique is however cumbersome and requires concurrent fluoroscopic imaging which led to the development of a new endoscopic functional luminal imaging probe (EndoFLIP). This probe consists of a $240-\mathrm{cm}$ catheter with a 14-cm bag mounted on its distal end. Inside the bag, 17 electrodes are spaced at $4 \mathrm{~mm}$ intervals. At the end of an upper gastrointestinal endoscopy, the EndoFLIP probe is introduced and placed across the EGJ after which the cylindrical bag is distended during real-time monitoring to ensure proper bag placement. The EndoFLIP technique uses impedance planimetry to determine multiple cross sectional areas (CSAs) within the cylindrical bag together with concurrent pressure measurements during volumecontrolled distensions. ${ }^{55,56}$ Assessment of intraluminal pressure along with the CSAs permits calculation of EGJ distensibility (i.e. narrowest CSA / intrabag pressure). Two studies used this novel EndoFLIP technique in achalasia patients and healthy controls, and demonstrated that newly diagnosed achalasia patients had reduced EGJ distensibility compared to healthy controls. In addition impaired EGJ distensibility after treatment was associated with poor esophageal emptying and treatment 
failure. ${ }^{57,58}$ These results together with the aim of improving EGJ distensibility in achalasia treatment led to the hypothesis that assessment of EGJ distensibility with the EndoFLIP technique might be useful to predict treatment outcome.

\section{Gastroesophageal reflux disease}

Gastroesophageal reflux disease (GERD) is defined as a disorder which develops when reflux of gastric contents causes troublesome symptoms and/or complications (e.g. esophagitis, Barrett's esophagus, and adenocarcinoma). ${ }^{59}$ Heartburn and regurgitation are considered cardinal symptoms of GERD, although patients may also present with extra-esophageal manifestations (e.g. cough, hoarseness). ${ }^{59}$

Gastroesophageal reflux disease is a multifactorial disorder. Delayed gastric emptying and impaired esophageal clearance through dysfunctional esophageal motility may contribute to GERD. ${ }^{60,61}$ However, dysfunction of the EGJ is considered the cardinal mechanism contributing to GERD. The EGJ forms the mechanical barrier between the esophagus and stomach and consists of the lower esophageal sphincter (LES) and crural diaphragm. Previous studies described an increased EGJ distensibility in GERD patients compared to healthy controls using a barostat device or EndoFLIP. ${ }^{52,56}$ Excessive EGJ distensibility is considered a primary pathophysiological abnormality in GERD patients as it is associated with increased volumes of gastroesophageal reflux and increased frequency of Transient Lower Esophageal Sphincter Relaxations (TLESRs). Transient Lower Esophageal Sphincter Relaxations are characterized by complete prolonged relaxations of the LES not caused by swallowing and mediated by a vagal reflex in response to gastric distension. ${ }^{62}$ Although the frequency of TLESRs is not different between GERD patients and healthy controls, TLESRs are more often associated with acid reflux in GERD patients. ${ }^{63}$ The thus resulting increased esophageal acid exposure induces mucosal damage, inflammation, and innervation of sensory nerve endings leading to symptom generation. ${ }^{64,65}$

Acid suppressive therapy is considered first-line treatment for patients with GERD. Although acid suppression is effective in the majority of patients, $30-40 \%$ of patients continue to experience GERD symptoms despite healing of esophagitis. For many years, surgical Nissen fundoplication has been the main alternative therapy for patients dissatisfied with acid suppressive medication. Although Nissen fundoplication is considered an effective treatment option in terms of reflux control, concerns have been raised with regard to its side-effect profile with negative impact on quality of life (e.g. gas-related symptoms, dysphagia). ${ }^{66-68}$ Consequently, a "therapy gap" exists for GERD patients dissatisfied with acid suppressive therapy and reluctant to undergo surgical fundoplication due to its potential side effects. ${ }^{38}$ In the past decades, several minimally invasive (endoscopic) treatment options have been developed to fill this "therapy gap" including radiofrequency energy delivery (Stretta, Mederi Therapeutics), sphincter augmentation of the LES by a magnetic implant (LINX, Torax 
Medical), and pacing of the LES by a surgically implanted pacemaker (EndoStim, EndoStim BV). ${ }^{69-73}$ Transoral Incisionless Fundoplication (TIF) with the Esophyx device is potentially the procedure most closely related to surgical fundoplication in order to improve EGJ distensibility. Although results of the first uncontrolled trials were promising, PPI- and sham-controlled randomized trials found beneficial effects in only subgroups of patients. ${ }^{11,74}$ A study by Witteman et al. described normalization of esophageal acid exposure time in only $29 \%$ of patients whereas $39 \%$ of patients were completely off of PPIs after 12 months follow-up. ${ }^{11,75}$ The beneficial short-term effect with less durable results compared to traditional surgical antireflux therapy seems applicable to almost all minimally invasive antireflux treatment modalities. As a result, selection of a subgroup of patients with potential beneficial effects of these treatment modalities seems warranted. ${ }^{38}$

As antireflux treatment is focused on improving EGJ barrier function by decreasing EGJ distensibility, assessment of this parameter with the novel EndoFLIP technique seems interesting. Previous studies described increased EGJ distensibility in GERD patients compared to healthy controls, and a decrease in distensibility after endoluminal, Nissen or Toupet fundoplication. ${ }^{56,76,77}$ Therefore, EGJ distensibility might potentially be a predictor for treatment outcome which might guide GERD patients to either minimally invasive (endoscopic) or surgical treatment modalities.

\section{Gastroparesis}

Gastroparesis is defined as a clinical syndrome of objectively delayed gastric emptying in the absence of a mechanical obstruction. Cardinal symptoms include postprandial fullness, early satiety, nausea, vomiting, bloating and upper abdominal pain. ${ }^{78}$ Postsurgical, diabetic and idiopathic gastroparesis are the most common associated conditions. The underlying pathophysiological mechanisms are still incompletely understood although impaired gastric accommodation, antral hypomotility, disordered antroduodenal coordination, pyloric dysfunction and visceral hypersensitivity might contribute to gastroparesis and symptom generation. ${ }^{79-82}$

Treatment of gastroparesis remains challenging and is focused on improving symptoms and nutritional status. ${ }^{83}$ Initial therapy consists of dietary recommendations with intake of small and frequent meals, and reduction of fat and fiber intake. Moreover, glycemic control should be improved in diabetic patients. ${ }^{84,85}$ Dietary advices are often combined with use of prokinetic agents such as dopaminergic-2-antagonists (e.g. domperidone and metoclopramide), or motilinanalogues. Erythromycin, a macrolide antibiotic, can be used as well for repeated short periods of approximately four weeks due to development of tachyphylaxis. ${ }^{78}$ However, lack of effectiveness and potential serious neurological or cardiac side effects limit the long-term use of prokinetic agents. ${ }^{86}$ 
In case symptoms are refractory to dietary adjustments and use of prokinetic agents, nutritional interventions are often necessary to maintain an adequate nutritional status (e.g. gastric rest with duodenal feeding, percutaneous endoscopic gastrostomy with jejunal extension) and more invasive (endoscopic) treatment options are considered including endoscopic pyloric botulinum toxin injection, transpyloric stent placement, endoscopic pyloromyotomy (G-POEM) and surgical pyloroplasty. ${ }^{78}$ These anatomically focused endoscopic and surgical interventions attempt to increase pyloric distensibility based on the pathophysiological mechanism of 'pylorospasm'. ${ }^{80}$ Wellington et al. recently described a subgroup of gastroparesis patients with a 'functional obstructive phenotype' with normal gastric myoelectrical activity which might be due to pyloric stenosis or pylorospasm. ${ }^{87}$ As pyloric therapies appear to be especially effective in this subgroup of gastroparesis patients, reliable assessment of pyloric function seems warranted in order to improve individualized therapy. Reliable assessment of pyloric function is however challenging because of the dynamic neuromuscular complexity and limitations of available diagnostic modalities. Although the novel EndoFLIP technique might be a promising tool for assessment of pyloric distensibility in future research, (high-resolution) antroduodenal manometry remains a more commonly available diagnostic modality for characterization of antral and pyloric activity.

\section{Aims and outline of this thesis}

\section{Part I Patient-reported outcome measures in functional dyspepsia}

The first part of this thesis (chapters 2 and 3) focuses on the use of PROMs for symptom assessment in patients with functional dyspepsia.

In chapter $\mathbf{2}$ we performed an extensive systematic literature search to identify available disease-specific PROMs for symptom assessment in patients with functional dyspepsia. Psychometric validation properties, strengths and limitations of all available PROMs are described, together with recommendations for use of PROMs in current clinical practice.

In chapter 3 we describe the development of a novel PROM based on the Experience Sampling Method as method for real-time symptom assessment. Patient focus groups and an international expert meeting were arranged to select a relevant set of items, and cognitive interviews were performed to evaluate patients' understanding. 


\section{Part II Prediction of treatment outcome in upper gastrointestinal disorders}

The second part of this thesis (chapters 4-7) focuses on the use of diagnostic modalities to predict treatment outcome in patients with several upper gastrointestinal disorders treated with (endoscopic) minimally invasive interventions.

In chapter $\mathbf{4}$ we evaluate the role of a novel diagnostic modality, an endoscopic functional luminal imaging probe (EndoFLIP), in the treatment of newly diagnosed achalasia patients. We compared distensibility of the esophagogastric junction (EGJ) between newly diagnosed achalasia patients and healthy controls, and evaluated the effect of pneumatic dilation on EGJ distensibility. Moreover, we explored the role of the EndoFLIP as potential predictor of treatment outcome.

In chapter $\mathbf{5}$ we report on an investigation of antireflux mechanisms in patients with gastroesophageal reflux disease (GERD) treated by Transoral Incisionless Fundoplication (TIF), an endoscopic alternative for conventional antireflux surgery. We focused on the effect of TIF on transient lower esophageal sphincter relaxations (TLESRs) and EGJ distensibility.

In chapter 6 we explored the role of the novel EndoFLIP technique in the preoperative diagnostic work-up for endoluminal antireflux surgery in patients with GERD.

In chapter 7 we evaluated the clinical effect of pyloric botulinum toxin treatment in patients with gastroparesis. Furthermore, we assessed whether high-resolution antroduodenal manometric and/or scintigraphic variables could predict treatment outcome.

In chapter 8, the general discussion, the findings of the various chapters are summarized and placed into perspective, integrating newly acquired knowledge and allowing an overview with future perspectives and new leads. 


\section{References}

1. Numens ME DWN, Dirven JAM et al. NHG-standaard Maagklachten. 2013.

2. NIVEL: incidents and prevalences of disorder in general practitioner practices. 2016.

3. Adang RP, Vismans JF, Talmon JL, Hasman A, Ambergen AW, Stockbrugger RW. Appropriateness of indications for diagnostic upper gastrointestinal endoscopy: association with relevant endoscopic disease. Gastrointest Endosc. 1995;42(5):390-7.

4. Koloski NA, Talley NJ, Boyce PM. Epidemiology and health care seeking in the functional GI disorders: a population-based study. Am J Gastroenterol. 2002;97(9):2290-9.

5. Stanghellini V, Chan FK, Hasler WL, Malagelada JR, Suzuki H, Tack J, et al. Gastroduodenal disorders. Gastroenterology. 2016;150(6):1380-92.

6. Ford AC, Marwaha A, Lim A, Moayyedi P. Systematic review and meta-analysis of the prevalence of irritable bowel syndrome in individuals with dyspepsia. Clin Gastroenterol Hepatol. 2010;8(5):401-9.

7. Camilleri $\mathrm{M}$, Halawi $\mathrm{H}$, Oduyebo I. Biomarkers as a diagnostic tool for irritable bowel syndrome: where are we? Expert Rev Gastroenterol Hepatol. 2017;11(4):303-16.

8. Sayuk GS. Editorial: functional dyspepsia treatment: trials and tribulations of targeted strategies. Am J Gastroenterol. 2017;112(6):910-2.

9. Arts J, Holvoet L, Caenepeel P, Bisschops R, Sifrim D, Verbeke K, et al. Clinical trial: a randomizedcontrolled crossover study of intrapyloric injection of botulinum toxin in gastroparesis. Aliment Pharmacol Ther. 2007;26(9):1251-8.

10. Friedenberg FK, Palit A, Parkman HP, Hanlon A, Nelson DB. Botulinum toxin A for the treatment of delayed gastric emptying. Am J Gastroenterol. 2008;103(2):416-23.

11. Witteman BP, Conchillo JM, Rinsma NF, Betzel B, Peeters A, Koek GH, et al. Randomized controlled trial of transoral incisionless fundoplication vs. proton pump inhibitors for treatment of gastroesophageal reflux disease. Am J Gastroenterol. 2015;110(4):531-42.

12. Moonen A, Boeckxstaens G. Finding the right treatment for achalasia treatment: risks, efficacy, complications. Curr Treat Options Gastroenterol. 2016;14(4):420-8.

13. Mahadeva S, Goh KL. Epidemiology of functional dyspepsia: a global perspective. World J Gastroenterol. 2006;12(17):2661-6.

14. Talley NJ, Ford AC. Functional Dyspepsia. N Engl J Med. 2015;373(19):1853-63.

15. Sander GB, Mazzoleni LE, Francesconi CF, Balbinotto G, Mazzoleni F, Wortmann AC, et al. Influence of organic and functional dyspepsia on work productivity: the HEROES-DIP study. Value Health. 2011;14(5 Suppl 1):S126-9.

16. Ford AC, Forman D, Bailey AG, Cook MB, Axon AT, Moayyedi P. Who consults with dyspepsia? Results from a longitudinal 10-yr follow-up study. Am J Gastroenterol. 2007;102(5):957-65.

17. Vanheel H, Vicario M, Vanuytsel T, Van Oudenhove L, Martinez C, Keita AV, et al. Impaired duodenal mucosal integrity and low-grade inflammation in functional dyspepsia. Gut. 2014;63(2):262-71.

18. Keely S, Walker MM, Marks E, Talley NJ. Immune dysregulation in the functional gastrointestinal disorders. Eur J Clin Invest. 2015;45(12):1350-9.

19. Di Stefano M, Miceli E, Tana P, Mengoli C, Bergonzi M, Pagani E, et al. Fasting and postprandial gastric sensorimotor activity in functional dyspepsia: postprandial distress vs. epigastric pain syndrome. Am J Gastroenterol. 2014;109(10):1631-9.

20. Karamanolis G, Caenepeel P, Arts J, Tack J. Association of the predominant symptom with clinical characteristics and pathophysiological mechanisms in functional dyspepsia. Gastroenterology. 2006;130(2):296-303.

21. Vanheel H, Carbone F, Valvekens L, Simren M, Tornblom H, Vanuytsel $T$, et al. Pathophysiological abnormalities in functional dyspepsia subgroups according to the Rome III Criteria. Am J Gastroenterol. 2017;112(1):132-40.

22. Vakil N, Halling K, Ohlsson L, Wernersson B. Symptom overlap between postprandial distress and epigastric pain syndromes of the Rome III dyspepsia classification. Am J Gastroenterol. 2013;108(5):767-74. 
23. Carbone F, Holvoet L, Tack J. Rome III functional dyspepsia subdivision in PDS and EPS: recognizing postprandial symptoms reduces overlap. Neurogastroenterol Motil. 2015;27(8):1069-74.

24. Moayyedi PM, Lacy BE, Andrews CN, Enns RA, Howden CW, Vakil N. ACG and CAG clinical guideline: management of dyspepsia. Am J Gastroenterol. 2017;112(7):988-1013.

25. (FDA) FaDA. Guidance for industry. Patient-reported outcome measures: use in medical product development to support labeling claims. 2009.

26. Shiffman S, Stone AA, Hufford MR. Ecological momentary assessment. Annu Rev Clin Psychol. 2008;4:1-32.

27. Stone AA, Shiffman S, Schwartz JE, Broderick JE, Hufford MR. Patient compliance with paper and electronic diaries. Control Clin Trials. 2003;24(2):182-99.

28. Shiffman S, Balabanis MH, Gwaltney CJ, Paty JA, Gnys M, Kassel JD, et al. Prediction of lapse from associations between smoking and situational antecedents assessed by ecological momentary assessment. Drug Alcohol Depend. 2007;91(2-3):159-68.

29. Myin-Germeys I, Oorschot M, Collip D, Lataster J, Delespaul P, van Os J. Experience sampling research in psychopathology: opening the black box of daily life. Psychol Med. 2009;39(9):1533-47.

30. van Os J, Verhagen S, Marsman A, Peeters F, Bak M, Marcelis $M$, et al. The experience sampling method as an mealth tool to support self-monitoring, self-insight, and personalized health care in clinical practice. Depress Anxiety. 2017;34(6):481-93.

31. Carbone F, Holvoet L, Vandenberghe A, Tack J. Functional dyspepsia: outcome of focus groups for the development of a questionnaire for symptom assessment in patients suffering from postprandial distress syndrome (PDS). Neurogastroenterol Motil. 2014;26(9):1266-74.

32. Talley NJ. Functional dyspepsia: new insights into pathogenesis and therapy. Korean J Intern Med. 2016;31(3):444-56.

33. Fang YJ, Liou JM, Chen CC, Lee JY, Hsu YC, Chen MJ, et al. Distinct aetiopathogenesis in subgroups of functional dyspepsia according to the Rome III criteria. Gut. 2015;64(10):1517-28.

34. Bisschops R, Karamanolis G, Arts J, Caenepeel P, Verbeke K, Janssens J, et al. Relationship between symptoms and ingestion of a meal in functional dyspepsia. Gut. 2008;57(11):1495-503.

35. Aro P, Talley NJ, Johansson SE, Agreus L, Ronkainen J. Anxiety is linked to new-onset dyspepsia in the Swedish population: a 10-year follow-up study. Gastroenterology. 2015;148(5):928-37.

36. Mak AD, Wu JC, Chan Y, Chan FK, Sung JJ, Lee S. Dyspepsia is strongly associated with major depression and generalised anxiety disorder - a community study. Aliment Pharmacol Ther. 2012;36(8):800-10.

37. Pinto-Sanchez MI, Ford AC, Avila CA, Verdu EF, Collins SM, Morgan D, et al. Anxiety and depression increase in a stepwise manner in parallel with multiple FGIDs and symptom severity and frequency. Am J Gastroenterol. 2015;110(7):1038-48.

38. Ganz RA. A Review of New Surgical and Endoscopic Therapies for gastroesophageal reflux disease. Gastroenterology Hepatology 2016;12(7):424-31.

39. Ates F, Vaezi MF. The pathogenesis and management of achalasia: current status and future directions. Gut Liver. 2015;9(4):449-63.

40. Vaezi MF, Pandolfino JE, Vela MF. ACG clinical guideline: diagnosis and management of achalasia. Am J Gastroenterol. 2013;108(8):1238-49; quiz 50.

41. Tuason J, Inoue H. Current status of achalasia management: a review on diagnosis and treatment. J Gastroenterol. 2017;52(4):401-6.

42. Kahrilas PJ, Boeckxstaens G. The spectrum of achalasia: lessons from studies of pathophysiology and high-resolution manometry. Gastroenterology. 2013;145(5):954-65.

43. Vaezi MF, Felix VN, Penagini R, Mauro A, de Moura EG, Pu LZ, et al. Achalasia: from diagnosis to management. Ann N Y Acad Sci. 2016;1381(1):34-44.

44. Boeckxstaens GE, Annese V, des Varannes SB, Chaussade S, Costantini M, Cuttitta A, et al. Pneumatic dilation versus laparoscopic Heller's myotomy for idiopathic achalasia. N Engl J Med. 2011;364(19):1807-16.

45. Moonen A, Annese V, Belmans A, Bredenoord AJ, Bruley des Varannes S, Costantini M, et al. Longterm results of the European achalasia trial: a multicentre randomised controlled trial comparing pneumatic dilation versus laparoscopic Heller myotomy. Gut. 2016;65(5):732-9. 
46. Inoue $\mathrm{H}$, Sato $\mathrm{H}$, Ikeda $\mathrm{H}$, Onimaru $\mathrm{M}$, Sato $\mathrm{C}$, Minami $\mathrm{H}$, et al. Per-oral endoscopic myotomy: a series of 500 patients. J Am Coll Surg. 2015;221(2):256-64.

47. Werner YB, Costamagna G, Swanstrom LL, von Renteln D, Familiari P, Sharata AM, et al. Clinical response to peroral endoscopic myotomy in patients with idiopathic achalasia at a minimum followup of 2 years. Gut. 2016;65(6):899-906.

48. Vaezi MF, Baker ME, Richter JE. Assessment of esophageal emptying post-pneumatic dilation: use of the timed barium esophagram. Am J Gastroenterol. 1999;94(7):1802-7.

49. Vaezi MF, Baker ME, Achkar E, Richter JE. Timed barium oesophagram: better predictor of long term success after pneumatic dilation in achalasia than symptom assessment. Gut. 2002;50(6):765-70.

50. Eckardt VF, Aignherr C, Bernhard G. Predictors of outcome in patients with achalasia treated by pneumatic dilation. Gastroenterology. 1992;103(6):1732-8.

51. Rohof WO, Lei A, Boeckxstaens GE. Esophageal stasis on a timed barium esophagogram predicts recurrent symptoms in patients with long-standing achalasia. Am J Gastroenterol. 2013;108(1):49-55.

52. Pandolfino JE, Shi G, Curry J, Joehl RJ, Brasseur JG, Kahrilas PJ. Esophagogastric junction distensibility: a factor contributing to sphincter incompetence. Am J Physiol Gastrointest Liver Physiol. 2002;282(6):G1052-8.

53. Pandolfino JE, Shi G, Trueworthy B, Kahrilas PJ. Esophagogastric junction opening during relaxation distinguishes nonhernia reflux patients, hernia patients, and normal subjects. Gastroenterology. 2003;125(4):1018-24.

54. Pandolfino JE, Curry J, Shi G, Joehl RJ, Brasseur JG, Kahrilas PJ. Restoration of normal distensive characteristics of the esophagogastric junction after fundoplication. Ann Surg. 2005;242(1):43-8.

55. McMahon BP, Drewes AM, Gregersen H. Functional oesophago-gastric junction imaging. World J Gastroenterol. 2006;12(18):2818-24.

56. Kwiatek MA, Pandolfino JE, Hirano I, Kahrilas PJ. Esophagogastric junction distensibility assessed with an endoscopic functional luminal imaging probe (EndoFLIP). Gastrointest Endosc. 2010;72(2):272-8.

57. Rohof WO, Hirsch DP, Kessing BF, Boeckxstaens GE. Efficacy of treatment for patients with achalasia depends on the distensibility of the esophagogastric junction. Gastroenterology. 2012;143(2):328-35.

58. Pandolfino JE, de Ruigh A, Nicodeme F, Xiao Y, Boris L, Kahrilas PJ. Distensibility of the esophagogastric junction assessed with the functional lumen imaging probe (FLIP) in achalasia patients. Neurogastroenterol Motil. 2013;25(6):496-501.

59. Vakil N, van Zanten SV, Kahrilas P, Dent J, Jones R, Global Consensus G. The Montreal definition and classification of gastroesophageal reflux disease: a global evidence-based consensus. Am J Gastroenterol. 2006;101(8):1900-20; quiz 43.

60. Stacher G, Lenglinger J, Bergmann H, Schneider C, Hoffmann M, Wolfl G, et al. Gastric emptying: a contributory factor in gastro-oesophageal reflux activity? Gut. 2000;47(5):661-6.

61. Simren M, Silny J, Holloway R, Tack J, Janssens J, Sifrim D. Relevance of ineffective oesophageal motility during oesophageal acid clearance. Gut. 2003;52(6):784-90.

62. Roman S, Holloway R, Keller J, Herbella F, Zerbib F, Xiao Y, et al. Validation of criteria for the definition of transient lower esophageal sphincter relaxations using high-resolution manometry. Neurogastroenterol Motil. 2017;29(2).

63. Trudgill NJ, Riley SA. Transient lower esophageal sphincter relaxations are no more frequent in patients with gastroesophageal reflux disease than in asymptomatic volunteers. Am J Gastroenterol. 2001;96(9):2569-74.

64. Farre R, Fornari F, Blondeau K, Vieth M, De Vos R, Bisschops R, et al. Acid and weakly acidic solutions impair mucosal integrity of distal exposed and proximal non-exposed human oesophagus. Gut. 2010;59(2):164-9.

65. Bredenoord AJ. Mechanisms of reflux perception in gastroesophageal reflux disease: a review. Am J Gastroenterol. 2012;107(1):8-15.

66. Oor JE, Roks DJ, Broeders JA, Hazebroek EJ, Gooszen HG. Seventeen-year outcome of a randomized clinical trial comparing laparoscopic and conventional Nissen fundoplication: a plea for patient counseling and clarification. Ann Surg. 2017;266(1):23-8.

67. Kessing BF, Broeders JA, Vinke N, Schijven MP, Hazebroek EJ, Broeders IA, et al. Gas-related symptoms after antireflux surgery. Surg Endosc. 2013;27(10):3739-47. 
68. Broeders JA, Roks DJ, Ahmed Ali U, Watson DI, Baigrie RJ, Cao Z, et al. Laparoscopic anterior 180degree versus nissen fundoplication for gastroesophageal reflux disease: systematic review and metaanalysis of randomized clinical trials. Ann Surg. 2013;257(5):850-9.

69. Lipka S, Kumar A, Richter JE. No evidence for efficacy of radiofrequency ablation for treatment of gastroesophageal reflux disease: a systematic review and meta-analysis. Clin Gastroenterol Hepatol. 2015;13(6):1058-67 e1.

70. Skubleny D, Switzer NJ, Dang J, Gill RS, Shi X, de Gara C, et al. LINX(R) magnetic esophageal sphincter augmentation versus Nissen fundoplication for gastroesophageal reflux disease: a systematic review and meta-analysis. Surg Endosc. 2017;31(8):3078-84.

71. Ganz RA, Edmundowicz SA, Taiganides PA, Lipham JC, Smith CD, DeVault KR, et al. Long-term outcomes of patients receiving a magnetic sphincter augmentation device for gastroesophageal reflux. Clin Gastroenterol Hepatol. 2016;14(5):671-7.

72. Kappelle WF, Bredenoord AJ, Conchillo JM, Ruurda JP, Bouvy ND, van Berge Henegouwen MI, et al. Electrical stimulation therapy of the lower oesophageal sphincter for refractory gastro-oesophageal reflux disease - interim results of an international multicentre trial. Aliment Pharmacol Ther. 2015;42(5):614-25.

73. Rodriguez L, Rodriguez P, Gomez B, Ayala JC, Oxenberg D, Perez-Castilla A, et al. Two-year results of intermittent electrical stimulation of the lower esophageal sphincter treatment of gastroesophageal reflux disease. Surgery. 2015;157(3):556-67.

74. Hakansson B, Montgomery M, Cadiere GB, Rajan A, Bruley des Varannes S, Lerhun M, et al. Randomised clinical trial: transoral incisionless fundoplication vs. sham intervention to control chronic GERD. Aliment Pharmacol Ther. 2015;42(11-12):1261-70.

75. Hunter JG, Kahrilas PJ, Bell RC, Wilson EB, Trad KS, Dolan JP, et al. Efficacy of transoral fundoplication vs omeprazole for treatment of regurgitation in a randomized controlled trial. Gastroenterology. 2015;148(2):324-33 e5.

76. Hoppo T, McMahon BP, Witteman BP, Kraemer SJ, O'Rourke RW, Gravesen F, et al. Functional lumen imaging probe to assess geometric changes in the esophagogastric junction following endolumenal fundoplication. J Gastrointest Surg. 2011;15(7):1112-20.

77. Kessing BF, Bredenoord AJ, Schijven MP, van der Peet DL, van Berge Henegouwen MI, Smout AJ. Longterm effects of anti-reflux surgery on the physiology of the esophagogastric junction. Surg Endosc. 2015;29(12):3726-32.

78. Camilleri M, Parkman HP, Shafi MA, Abell TL, Gerson L, American College of Gastroenterology. Clinical guideline: management of gastroparesis. Am J Gastroenterol. 2013;108(1):18-37; quiz 8.

79. Camilleri M, Brown ML, Malagelada JR. Relationship between impaired gastric emptying and abnormal gastrointestinal motility. Gastroenterology. 1986;91(1):94-9.

80. Mearin F, Camilleri M, Malagelada JR. Pyloric dysfunction in diabetics with recurrent nausea and vomiting. Gastroenterology. 1986;90(6):1919-25.

81. Gourcerol G, Tissier F, Melchior C, Touchais JY, Huet E, Prevost G, et al. Impaired fasting pyloric compliance in gastroparesis and the therapeutic response to pyloric dilatation. Aliment Pharmacol Ther. 2015;41(4):360-7.

82. Langworthy J, Parkman HP, Schey R. Emerging strategies for the treatment of gastroparesis. Expert Rev Gastroenterol Hepatol. 2016;10(7):817-25.

83. Liu N, Abell T. Gastroparesis updates on pathogenesis and management. Gut Liver. 2017.

84. Tack J, Vanormelingen C. Management of gastroparesis: beyond basics. Curr Treat Options Gastroenterol. 2014;12(4):468-77.

85. Olausson EA, Storsrud S, Grundin H, Isaksson M, Attvall S, Simren M. A small particle size diet reduces upper gastrointestinal symptoms in patients with diabetic gastroparesis: a randomized controlled trial. Am J Gastroenterol. 2014;109(3):375-85.

86. Ukleja A, Tandon K, Shah K, Alvarez A. Endoscopic botox injections in therapy of refractory gastroparesis. World J Gastrointest Endosc. 2015;7(8):790-8.

87. Wellington J, Scott B, Kundu S, Stuart P, Koch KL. Effect of endoscopic pyloric therapies for patients with nausea and vomiting and functional obstructive gastroparesis. Auton Neurosci. 2017;202:56-61. 
Part 1

Patient-reported outcome measures in functional dyspepsia 



\section{Chapter 2}

\section{Systematic review: disease-specific instruments to assess gastrointestinal symptoms in functional dyspepsia}

F.G.M. Smeets, A.A.M. Masclee, J.M. Conchillo, D. Keszthelyi Neurogastroenterol Motil. 2018 [Epub ahead of print] 


\section{Abstract}

\section{Background}

Functional dyspepsia (FD) is a common functional gastrointestinal disorder with incompletely understood pathophysiology and heterogeneous symptom presentation. Assessment of treatment efficacy in FD is a methodological challenge as response to treatment must be assessed primarily by measuring subjective symptoms. Therefore, use of patient-reported outcome measures (PROMs) is recommended by regulatory authorities to assess gastrointestinal symptoms in clinical trials for functional dyspepsia. In the last decades a multitude of outcome measures has been developed. However, currently no PROM has been approved by the regulatory authorities and no consensus has been reached with regard to the most relevant outcome measure in FD.

\section{Purpose}

This systematic review discusses the available disease-specific outcome measures for assessment of FD symptoms with psychometric validation properties, strengths and limitations. Moreover, recommendations for use of current available outcome measures are provided and potential areas of future research are discussed. 


\section{Introduction}

Functional dyspepsia (FD) is a common functional gastrointestinal disorder defined by the presence of symptoms thought to originate from the gastroduodenal region in the absence of any organic, systemic or metabolic disease that is likely to explain the symptoms. ${ }^{1}$ Although the exact pathophysiology of functional dyspepsia is still incompletely understood, it is thought to be multifactorial in origin with involvement of gastroduodenal motor and sensory dysfunction, low-grade inflammation of the duodenal mucosa, and impaired mucosal integrity. ${ }^{1-4}$ Dyspeptic patients can present with a variety of symptoms and four FD core symptoms have been defined according to the Rome IV criteria including postprandial fullness, early satiation, epigastric burning and epigastric pain. ${ }^{5}$ However, excessive belching, postprandial nausea and upper abdominal bloating are considered important additional symptoms. ${ }^{1,5}$ Due to the heterogeneity of symptoms in patients with functional dyspepsia, it was hypothesized that different pathophysiological mechanisms could underlie different dyspeptic symptoms. Therefore, subdivision of patients into two diagnostic categories was proposed by the Rome IV criteria: (1) postprandial distress syndrome (PDS; presence of postprandial fullness and/or early satiety) and (2) epigastric pain syndrome (EPS; presence of epigastric pain and/or epigastric burning). ${ }^{1,6}$ The rationale for dividing patients into PDS and EPS subgroups was that classification might help to guide therapy i.e. prokinetics for patients with PDS and acid suppressive medication for patients with EPS. ${ }^{6,7}$ However, treatment of FD remains unsatisfactory in many patients for several reasons. First, development of novel therapies is hindered by the incompletely understood multifactorial pathophysiology. Moreover, assessment of treatment efficacy in FD is a methodological challenge as response to treatment must be assessed primarily by measuring subjective symptoms. The Food and Drug Administration (FDA) Guidance recommends the use of well-defined patient-reported outcome measures (PROMs) to assess treatment outcome. ${ }^{8}$ Patient-reported outcome measures should be reliable, valid, and responsive with an acceptable recall period. ${ }^{8}$ Although a multitude of questionnaires has been developed for assessment of FD symptoms, currently no instrument has undergone all development steps as recommended by the FDA. Therefore, no consensus has been reached with regard to the most relevant outcome measure in FD patients. As a consequence, recent studies used different questionnaires to evaluate treatment efficacy in FD patients which makes comparison of results difficult and prevents formulation of firm treatment recommendations. ${ }^{9,10}$

The aim of this systematic analysis is to review the published literature on methodology for assessment of gastrointestinal symptoms in FD patients. We sought to answer the following questions:

1. Which disease-specific questionnaires for assessment of gastrointestinal symptoms in patients with functional dyspepsia are currently available? 
2. What are the psychometric properties of these assessment tools, are they eligible for monitoring efficacy of treatment in patients with functional dyspepsia, and which questionnaire can be recommended?

\section{Methods}

\section{Search strategy}

To identify relevant articles, a structured search in the PubMed, Cochrane and Embase databases was performed with the following search strategy: (dyspepsia) AND (questionnaire OR outcome measure OR symptom assessment). English was used as filter and articles were selected between June 1970 and September 2017. The reference list of selected articles was examined to identify additional relevant citations.

Title and abstract of all articles were screened by two independent researchers (F.S. and D.K.) to assess eligibility according to pre-defined criteria. Full-text review was performed for studies reporting on psychometric validation properties (e.g. reproducibility, validity and responsiveness) of disease-specific patient-reported outcome measures (PROMs) for patients with (functional) dyspepsia. Although we intended to include only disease-specific outcome measures, frequently used but nonspecific questionnaires were included to provide a complete overview and thereby give accurate recommendations for clinical practice. Studies were excluded if they were reviews, comments or replies on original articles, or abstracts without available full text (presented at conferences). Moreover, studies with another aim (e.g. questionnaires used to diagnose dyspepsia) or studies evaluating only quality of life were excluded. Both reviewers agreed on inclusion of the articles. In case of disagreement, a third reviewer decided with regard to the inclusion (A.M.).

\section{Outcome measures}

The outcomes of interest with regard to this systematic review were (1) the available disease-specific patient-reported outcome measures used to assess gastrointestinal symptoms in FD patients, as well as (2) the psychometric properties, strengths and limitations of these PROMs.

In clinical trials with FD patients, several types of disease-specific outcome measures have been used. Disease-specific instruments can be divided into unidimensional (i.e. evaluating gastrointestinal symptoms) or multidimensional (i.e. evaluating gastrointestinal symptoms as well as other domains such as quality of life or impact of symptoms on emotional functioning) instruments. ${ }^{11}$ 
Psychometric validation of a patient-reported outcome measure incorporates assessment of reliability, validity and responsiveness to change. ${ }^{8}$ Reliability refers to the ability of a PRO instrument to yield consistent, reproducible results and is assessed by the test-retest reliability (i.e. indicates the stability of scores over time when no change in health status is expected) and internal consistency (i.e. indicates the correlation between different items comprising a scale of the instrument). Testretest reliability is often expressed by the intra class correlation coefficient (ICC) and an ICC above 0.75 is considered adequate. ${ }^{12-14}$ Cronbach's $\alpha$ is frequently used to describe internal consistency and a value between 0.7-0.9 is considered acceptable. ${ }^{8,13,14}$ Validity can be divided into different subtypes. Content validity describes the extent to which the instrument measures all symptoms indicative of FD, whereas construct validity provides evidence for the degree of correlation between related instruments measuring the same symptoms. Construct validity is expressed by the Pearson or Spearmann correlation coefficient referred to as " $r$ " and a correlation coefficient between 0.4-0.8 is considered appropriate. ${ }^{8}$ Known groups validity reflects the ability of a PROM to differentiate between groups which would theoretically be expected to differ in symptom scores. Responsiveness of the instrument is the ability to detect clinically meaningful changes in symptoms over time. Several methods are used for assessment of responsiveness. First, the (significant) change in symptom scores after treatment can be assessed. Moreover, effect sizes (ES) and standardized response means (SRMs) can be measured. The ES estimates the change in symptom score $(\delta)$ relative to the variability of the symptom score at baseline (i.e. ES $=\delta$ symptom score / standard deviation symptom score at baseline). Outcome measures with ES $>0.8$ are considered responsive to change. ${ }^{15}$ The standardized response mean (SRM) is calculated by dividing the change in symptom score $(\delta)$ between the standard deviation of the change (i.e. SRM $=\delta$ symptom score / standard deviation of the change). Outcome measures with SRM $>0.8$ are considered responsive to change. ${ }^{16}$

Besides psychometric validation properties, we evaluated the following characteristics of PROMs for assessment of strengths and limitations: (1) whether validation was performed in the target population (i.e. patients with FD according to Rome criteria), (2) recall period, (3) assessment of FD core symptoms (Rome IV; i.e. postprandial fullness, early satiation, epigastric pain and epigastric burning) and additional dyspeptic symptoms (i.e. nausea, bloating and belching), and (4) available minimum clinically important difference (MCID).

Data extraction was performed by two independent researchers (F.S. and D.K.). In case of disagreement, differences were discussed and resolved. 


\section{Results}

The systemic literature search and screening of reference lists of selected articles identified 4304 citations after removal of duplicates. After abstract and full-text review, 40 articles were included in this systematic review for analysis of diseasespecific outcome measures. (Figure 2.1) The disease-specific outcome measures included in this review are divided into two categories: (1) unidimensional measures $(n=14)$ and $(2)$ multidimensional measures $(n=6)$. Table 2.1 provides a short overview of the available outcome measures.

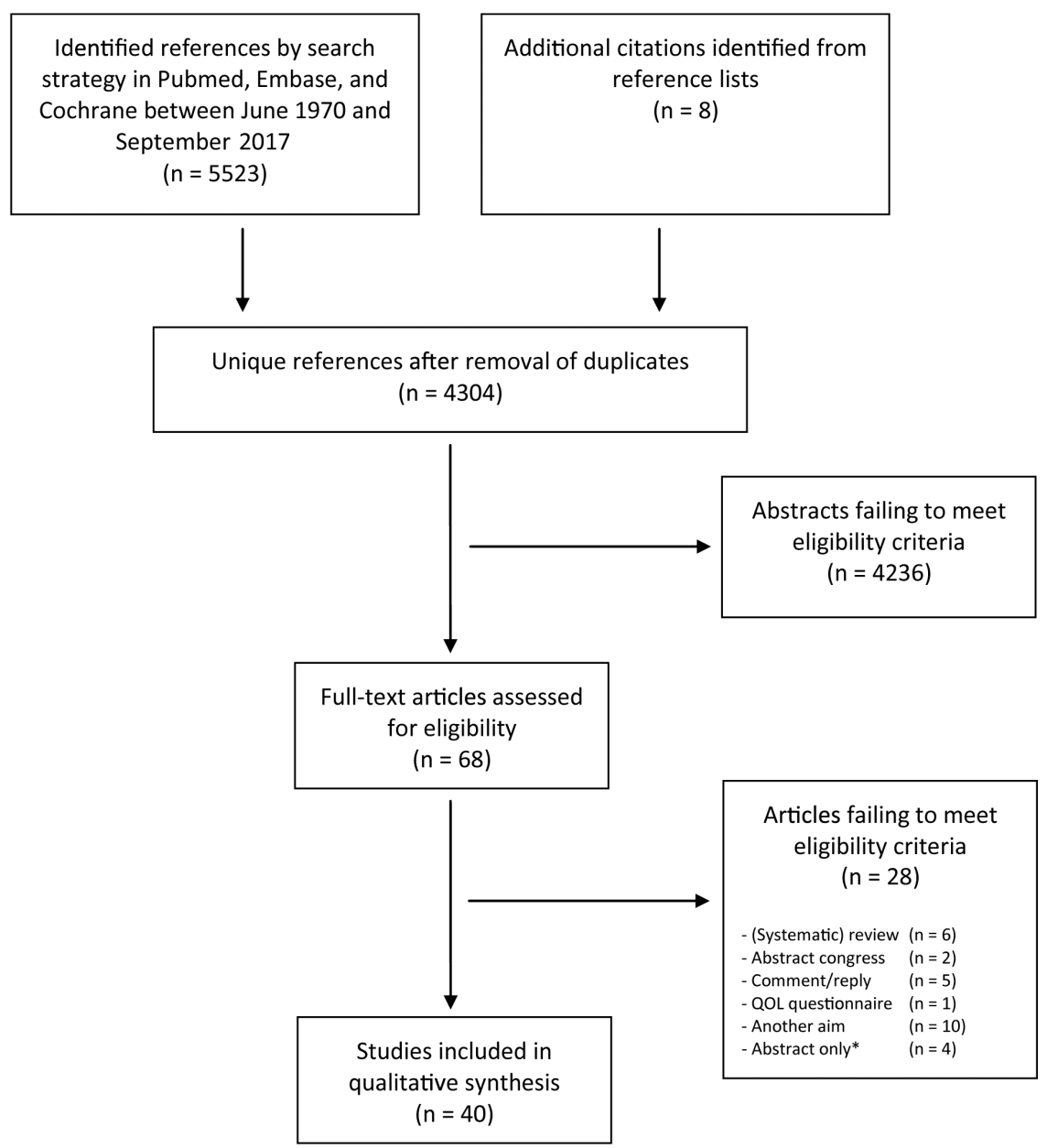

Figure 2.1 Flow diagram showing the studies included in the systematic literature review.

$\mathrm{QOL}=$ quality of life questionnaire. ${ }^{*}$ Abstract only: full text not available after contacting corresponding author and/or journal. 


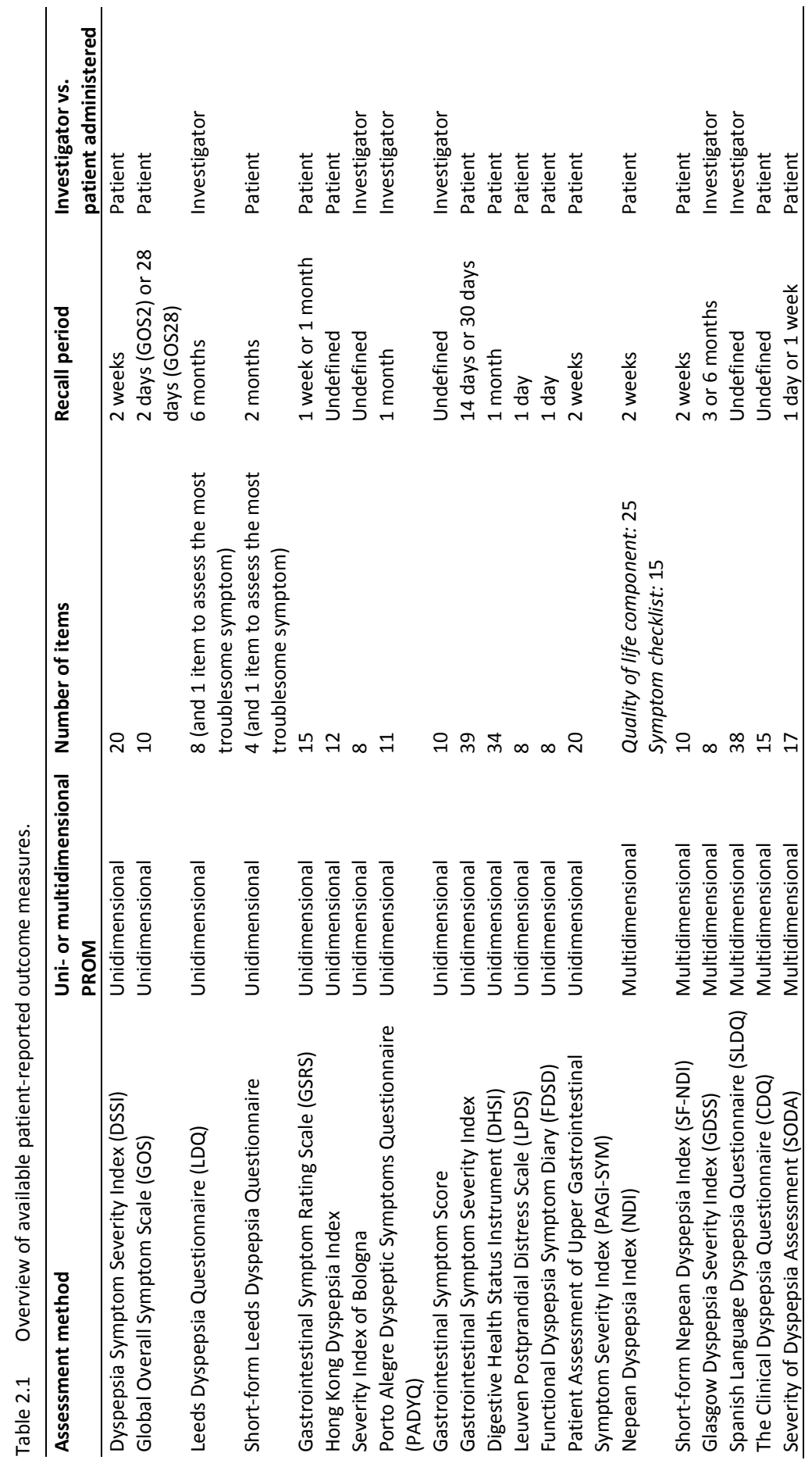


Extensive descriptions of all individual questionnaires are available as supplementary data. In short, we summarize here the following aspects examined:

\section{- Reliability}

Internal consistency and/or test-retest reliability were assessed in the majority of questionnaires with moderate to acceptable results (Cronbach's $\alpha$ ranging from 0.43 till 0.96; Intra class correlation coefficient (ICC) ranging from 0.42 till 0.96). Test-retest reliability was considered too low for the Global Overall Symptom Scale, several domains of the Gastrointestinal Symptom Rating Scale (GSRS), and Severity Index of Bologna. Four outcome measures assessed only internal consistency without testretest reliability, including the Digestive Health Status Instrument, Short-Form Nepean Dyspepsia Index, Functional Dyspepsia Symptom Diary, and Severity of Dyspepsia Assessment (SODA). ${ }^{17-21}$ As test-retest reliability is considered the most important type of reliability for PRO instruments used in clinical trials, there is insufficient evidence for adequate reliability of these four outcome measures. ${ }^{8}$ For several investigatoradministered questionnaires including the Leeds Dyspepsia Questionnaire (LDQ) and Glasgow Dyspepsia Severity Index (GDSS) both intra-rater and interrater reliability were assessed and showed good to excellent results. ${ }^{22,23}$

\section{- $\quad$ Validity}

Content validity was assessed by ten of the described questionnaires and was considered good by either experts in the field or by patients during focus groups or cognitive interviews. Moreover, good known groups validity was described in twelve of the questionnaires including the recently developed Functional Dyspepsia Symptom Diary. Widely varying results with regard to construct validity have been described for individual questionnaires, although the majority was correlated with subscales of either the short-form 36 health survey (SF-36) or the Patient Assessment of Upper Gastrointestinal Symptom Severity Index (PAGI-SYM).

One commonly used questionnaire is the Nepean Dyspepsia Index (NDI) which was developed as disease-specific quality of life (QOL) measure but also contains a symptom checklist for assessment of gastrointestinal symptoms. ${ }^{24}$ Although known groups validity has been assessed for both components of the Nepean Dyspepsia Index, construct validity has not been assessed for the symptom checklist. Moreover, construct validity of the short-form Nepean Dyspepsia Index was only assessed by correlating scores with the Nepean Dyspepsia Index but not with independent gastrointestinal questionnaires. ${ }^{20}$

\section{- Responsiveness}

Assessment of responsiveness is considered essential for use of outcome measures in clinical trials. Although responsiveness was adequate for the majority of questionnaires, it was not yet assessed for five outcome measures (e.g. Functional 
Dyspepsia Symptom Diary, Dyspepsia Symptom Severity Index, Gastrointestinal Symptom Severity Index, Spanish Language Dyspepsia Questionnaire, and The Clinical Dyspepsia Questionnaire) which limits the use of these outcome measures in clinical trials. ${ }^{17-19,25-28}$

The Leuven Postprandial Distress Scale (LPDS) is a recently developed outcome measure for FD patients primarily with the PDS subtype. Assessment of responsiveness was performed in 60 patients according to the Rome III criteria (PDS: 48\% and PDS-EPS overlap: 52\%). Whereas responsiveness was demonstrated for patients with PDS symptoms, no correlation was found between the overall treatment evaluation and change of symptom scores for patients with EPS symptoms. This might be due to the more prevalent and more severe PDS symptoms of included patients.

\section{Additional characteristics}

\section{Validation in target population}

Of the available PROMs, only the Leuven Postprandial Distress Scale and Functional Dyspepsia Symptom Diary have been validated in FD patients according to Rome III criteria.

Investigator versus patient administration

Six outcome measures are investigator- instead of patient-administered (i.e. Leeds Dyspepsia Questionnaire, Severity Index of Bologna, Porto Alegre Dyspeptic Symptoms Questionnaire, Gastrointestinal Symptom Score, Glasgow Dyspepsia Severity Index, and Spanish Language Dyspepsia Questionnaire).

\section{Recall period}

The recall period ranges between one day and six months, whereas the recall period has been undefined for five PROMs (i.e. Hong Kong Dyspepsia Index, Severity Index of Bologna, Gastrointestinal Symptom Score, Spanish Language Dyspepsia Questionnaire, and The Clinical Dyspepsia Questionnaire). Although no optimal recall period has been defined for functional dyspepsia, the FDA recommends daily assessment of symptoms in functional gastrointestinal disorders due to symptom variability. ${ }^{5,8,29}$ Only the Leuven Postprandial Distress Scale, Functional Dyspepsia Symptom Diary and modified Severity of Dyspepsia Assessment ( $m S O D A$ ) have a recall period of 24 hours.

Core symptoms of functional dyspepsia

Assessment of all four FD core symptoms according to the Rome IV criteria is limited to four PROMs (i.e. Dyspepsia Symptom Severity Index, Leuven Postprandial Distress 
Scale, symptom checklist of the Nepean Dyspepsia Index, and Functional Dyspepsia Symptom Diary).

Minimum clinically important difference (MCID)

A minimum clinically important difference (MCID) is available for five outcome measures (i.e. Gastrointestinal Symptom Rating Scale, Leuven Postprandial Distress Scale, Patient Assessment of Upper Gastrointestinal Symptom Severity Index, Nepean Dyspepsia Index, and modified Severity of Dyspepsia Assessment).

\section{Discussion}

In this systematic review, the currently available disease-specific instruments for assessment of symptoms in patients with functional dyspepsia have been evaluated. Although development of a patient-reported outcome measure is necessary to uniformly assess FD symptoms in clinical trials for evaluation of treatment efficacy, no universally accepted PROM is currently available.

\section{Development of PROMs}

Development of a PROM is a comprehensive process and consists of several steps. Regulatory authorities (FDA) suggest performing a literature review and obtaining expert opinion, followed by patient focus groups and cognitive debriefing. Input from the target population is essential for item generation, and patient focus group interviews were performed in the development of several PROMs (e.g. Leuven Postprandial Distress Scale, Functional Dyspepsia Symptom Diary, Dyspepsia Symptom Severity Index, and Nepean Dyspepsia Index).

\section{Psychometric validation of PROMS}

Psychometric validation has to be performed evaluating reliability, validity and responsiveness. ${ }^{8}$ The majority of the described questionnaires proved to be reliable and valid. The lower test-retest reliability for the abdominal pain domain of the Gastrointestinal Symptom Rating Scale might be due to the fact that this domain includes only two items. One also has to take into account the complex assessment of pain due to symptom variability. For instance, for the Global Overall Symptom Scale 28 , test-retest reliability was assessed with an interval of 56 days which might be too long for accurate assessment of 'stable' gastrointestinal symptoms.

In order to evaluate treatment efficacy in clinical trials, a PROM should be responsive to change. Responsiveness of the Leuven Postprandial Distress Scale has only been demonstrated for PDS symptoms, not for EPS. ${ }^{30}$ Moreover, psychometric 
validation has to be performed in a population of functional dyspepsia patients and currently only the Leuven Postprandial Distress Scale and Functional Dyspepsia Symptom Diary have been evaluated in FD patients according to the Rome III criteria. ${ }^{8,19,29,30}$ Therefore, the majority of the instruments we evaluated do not fulfill all psychometric validation criteria as defined by the FDA. ${ }^{31}$

\section{Patient vs. investigator administered PROMs}

The FDA defines a PROM as any report of the status of a patient's health condition that comes directly from the patient without interpretation of the patient's response by a clinician. ${ }^{8}$ Patient self-completion of a PROM is recommended to reduce social desirability bias. ${ }^{11}$ In addition, recent studies described poor to moderate overall agreement between patients' and expert clinicians' rating of symptom severity in FD patients, and underestimation of several symptoms (e.g. early satiety and abdominal pain) by clinicians which favours the use of a patient-administered outcome measure. ${ }^{19,32,33}$ This might limit the use of the Leeds Dyspepsia Questionnaire, Porto Alegre Dyspeptic Symptoms Questionnaire, Gastrointestinal Symptom Score and Glasgow Dyspepsia Severity Index as these are investigator-administered instruments. ${ }^{22,23,34,35}$

\section{Functional dyspepsia: core and additional symptoms}

Another challenge for development of a PROM is the multifactorial nature of functional dyspepsia and the diverse symptom presentation of patients. As the FDA states that in functional disorders the effect of treatment should be measured on each symptom in order to ensure that treatment does not negatively affect symptoms, we suggest that a PROM should evaluate at least the four FD core symptoms (early satiation, postprandial fullness, epigastric burning and epigastric pain) as well as the three additional symptoms (upper abdominal bloating, postprandial nausea and excessive belching). ${ }^{5,8,36}$ The latter three were reported by a substantial subgroup of FD patients during patient focus group interviews, cognitive interviews and validation studies (i.e. bloating: 33-93\%, nausea: 31-73\%, belching: 27-69\%). 1,5,30,37 Interestingly, during development of the Functional Dyspepsia Symptom Diary, dyspeptic patients ranked 'bloating' - which is not a core symptom according to Rome IV - as the most important symptom for improvement with effective therapy. ${ }^{37}$ Consequently, the total symptom score of the Functional Dyspepsia Symptom Diary consists of the scores for the four Rome IV core FD symptoms as well as for bloating, with the other two additional symptoms of belching and nausea being evaluated separately as individual items. Currently, no quantitative data is available for direct comparison of the validity, reliability and responsiveness with assessment of four vs. five or seven FD symptoms by PROMs. Therefore, until 
such data becomes available, we favor assessment of the four core symptoms and the three additional symptoms.

\section{Limitations of retrospective outcome measures}

For accurate assessment of FD core symptoms, an accurate recall period should be used to prevent recall bias as the available retrospective questionnaires are based on autobiographical memory. ${ }^{38}$ Only the Leuven Postprandial Distress Scale, Functional Dyspepsia Symptom Diary and modified Severity of Dyspepsia Assessment have a recall period of 24 hours, in accordance with the FDA recommendation.

Besides recall bias, two additional limitations of retrospective questionnaires should be considered. First, symptom variability in functional disorders might be due to the influence of triggers and circumstances. For example, FD symptoms might be influenced by intake of food (e.g. fat) and psychological factors (e.g. anxiety)..$^{39-41}$ Therefore, ecological bias can occur when questionnaires are completed in another environment or situation compared to situations in which symptoms were triggered. Furthermore, lack of patient compliance is another major limitation of paper questionnaires. This limitation can potentially be eliminated by the use of electronic instruments. $^{42}$

\section{Recommendation for use of current available instruments}

In 2011, Ang et al. published an extensive review about PROMs for functional dyspepsia. None of the available PROMs was considered appropriate for use in clinical trials which warranted the urgent need for development and validation of novel outcome measures. Since 2011, new PROMs have become available (i.e. Leuven Postprandial Distress Scale, Functional Dyspepsia Symptom Diary (FDSD), Gastrointestinal Symptom Severity Index) which have been included in the present systematic review. However, neither a universally validated and accepted patientreported-outcome measure for assessment of treatment efficacy in clinical trials of FD patients, nor a FD-specific recommendation of the FDA are currently available. Therefore, recent clinical trials used different questionnaires and methods to assess treatment efficacy including the Nepean Dyspepsia Index, daily symptom diaries, a binary outcome measure (relief or no relief of symptoms), PAGI-SYM, and assessment of overall treatment effect. ${ }^{9,10,43-45}$ Therefore, selection of one or a limited number of current available outcome measures is necessary to uniformly assess FD symptoms in clinical trials. The question arises which questionnaires should be recommended based on the results of the current systematic review, pending the proper validation of novel PROMs.

The Leuven Postprandial Distress Scale and Functional Dyspepsia Symptom Diary appear to be promising outcome measures in FD patients as item selection was 
performed by patient focus groups and/or cognitive debriefing, the questionnaires are patient-completed, contain all FD core and additional dyspeptic symptoms, and have a short recall period of 24 hours. For the recently developed Functional Dyspepsia Symptom Diary only preliminary data are available with regard to psychometric validation. Although good internal consistency and 'known-groups' validity were described, additional research is necessary to evaluate test-retest reliability, construct validity and responsiveness before use of this PROM in clinical trials. The Leuven Postprandial Distress Scale demonstrated good reliability, validity and responsiveness for the PDS domain. Additional studies are necessary to validate use of the Leuven Postprandial Distress Scale in patients with EPS predominant symptoms. In the case that psychometric validation for EPS symptoms is confirmed for (a modified version of) the Leuven Postprandial Distress Scale and additional psychometric validation is performed for the Functional Dyspepsia Symptom Diary, these PROMs might prove to be excellent outcome measures to evaluate the full range of FD symptoms, as recommended by the FDA.

The use of a single universal questionnaire for assessment of the full range of FD symptoms seems favourable for several reasons. First, previous studies demonstrated a high percentage of patients fulfilling both criteria for EPS and PDS ranging from 34.4 till $66 \% .^{30,46-49}$ In addition, the subdivision of patients into PDS and EPS subtypes was proposed to serve as guide for diagnostic and therapeutic approaches as it was hypothesized that distinct pathophysiological processes were present between the two subtypes. Current evidence for the presence of distinct etiopathogenesis is limited and inconclusive. Previous studies found no difference in gastric accommodation between the two FD subtypes and contradictory results were obtained with regard to gastric hypersensitivity and gastric emptying. ${ }^{50,51}$ Division of FD patients into subtypes does currently not reliably distinguish subgroups with a different underlying pathophysiology. The use of this classification to guide therapy is therefore limited and favors use of a single universal PROM for assessment of all FD core symptoms. ${ }^{2,52-55}$

Apart from the Leuven Postprandial Distress Scale and Functional Dyspepsia Symptom Diary, two questionnaires assess all FD core symptoms: (1) the symptom checklist of the Nepean Dyspepsia Index and (2) the Dyspepsia Symptom Severity Index. ${ }^{5}$ Use of the Dyspepsia Symptom Severity Index in clinical trials for the evaluation of treatment efficacy is limited by the fact that responsiveness has never been evaluated. $^{25}$ The Nepean Dyspepsia Index has widely been used and is considered a valid, reliable and responsive questionnaire for assessment of FD symptoms with a recall period of two weeks. However, it is important to consider that the Nepean Dyspepsia Index measures quality of life, whereas the symptom checklist assesses the severity and frequency of dyspeptic symptoms. Although psychometric validation has been performed for the Nepean Dyspepsia Index, test-retest reliability and convergent validity have never been assessed for the separate symptom checklist. ${ }^{20,24,56,57}$ 


\section{Future perspectives}

\section{Patient-reported outcome measurement system (PROMIS)}

In 2004, the National Institute of Health (NIH) has launched the Patient-Reported Outcome Measurement System (PROMIS) to develop a set of short, computer-based and reliable PROMs to assess physical, mental and social health for research purposes and clinical care. Short outcome measures for eight gastrointestinal symptom complexes were developed with good reliability and validity. ${ }^{58,59}$ Although use of these domain-specific PROMs was hypothesized to improve patient satisfaction, a recent performed trial compared use of PROMIS with regular care and found no difference in patient satisfaction and shared decision making. ${ }^{60}$ However, several limitations should be considered including the poor response rate, and lack of information about influence on clinic visit efficiency and frequency, cost-effectiveness and long-term effects which demonstrates the need for additional research. ${ }^{60,61}$

\section{Experience Sampling Method (ESM)}

Real-time assessment of symptoms is recommended to minimize recall bias, and use of an e-health approach seems favourable to improve patient comfort and reduce non-compliance. The Experience Sampling Method (ESM) is a digital method used for 'real-time symptom assessment' which has been validated in psychological studies. ${ }^{62}$ Repeated measures are used to assess variability in symptoms, and might provide information about potential triggers as it takes into account situations and circumstances (e.g. meal intake, social environment, psychological factors) that have occurred prior to symptom generation which might reduce ecological bias. Previous studies described associations between functional dyspepsia, anxiety and depression. Assessment of psychological factors, besides gastrointestinal symptoms, might therefore provide insight into psychological triggers with potential treatment consequences. Inclusion of pictograms, besides verbal descriptors, might be another advantage of the digital ESM. Recently, Tack et al. developed a set of pictograms to accompany verbal descriptors for dyspeptic symptoms. ${ }^{19}$ Use of pictograms enhanced concordance of FD symptom rating by patients compared to physicians' symptom evaluation. ${ }^{19}$ Addition of pictograms might therefore be a useful adjunctive for development of an ESM-based PROM. ${ }^{29}$ One potential relevant limitation of the ESM might be a higher patient burden due to the repeated measures at random moments during daily life. Use of a restricted number of questions might reduce this limitation. $^{63}$

Use of the ESM in patients with gastrointestinal diseases is currently limited. Two studies evaluated the use of ESM in IBS patients and demonstrated that patients reported higher scores for abdominal pain in retrospective questionnaires compared 
to mean scores derived with ESM. ${ }^{64,65}$ To our knowledge, use of the ESM in patients with FD has not been described previously. Therefore, extensive item selection and psychometric validation of the ESM in FD patients has to be performed.

\section{Conclusion}

In conclusion, multiple different questionnaires are currently used in clinical trials to assess gastrointestinal symptoms in patients with functional dyspepsia. Due to the lack of a validated and widely accepted PROM, results of clinical trials are difficult to compare demonstrating the need for a PROM. Although current available questionnaires do not fulfill all criteria for psychometric validation, the Leuven Postprandial Distress Scale and Functional Dyspepsia Symptom Diary seem most promising. However, retrospective questionnaires have several limitations which might potentially be reduced by the use of novel digital approaches such as the Experience Sampling Method which seems a promising method for use in patients with functional gastrointestinal disorders. 


\section{References}

1. Stanghellini V, Chan FK, Hasler WL, Malagelada JR, Suzuki H, Tack J, et al. Gastroduodenal Disorders. Gastroenterology. 2016;150(6):1380-92.

2. Vanheel H, Vicario M, Vanuytsel T, Van Oudenhove L, Martinez C, Keita AV, et al. Impaired duodenal mucosal integrity and low-grade inflammation in functional dyspepsia. Gut. 2014;63(2):262-71.

3. Keely S, Walker MM, Marks E, Talley NJ. Immune dysregulation in the functional gastrointestinal disorders. Eur J Clin Invest. 2015;45(12):1350-9.

4. Carbone F, Tack J. Gastroduodenal mechanisms underlying functional gastric disorders. Dig Dis. 2014;32(3):222-9.

5. Taylor F, Reasner DS, Carson RT, Deal LS, Foley C, lovin R, et al. Development of a symptom-based patient-reported outcome instrument for functional dyspepsia: a preliminary conceptual model and an evaluation of the adequacy of existing instruments. Patient. 2016;9(5):409-18.

6. Talley NJ, Ford AC. Functional Dyspepsia. N Engl J Med. 2015;373(19):1853-63.

7. Vanheel H, Tack J. Therapeutic options for functional dyspepsia. Dig Dis. 2014;32(3):230-4.

8. (FDA) FaDA. Guidance for industry; patient-reported outcome measures: use in medical product development to support labeling vlaims 2009.

9. Talley NJ, Locke GR, Saito YA, Almazar AE, Bouras EP, Howden CW, et al. Effect of amitriptyline and escitalopram on functional dyspepsia: a multicenter, randomized controlled study. Gastroenterology. 2015;149(2):340-9 e2.

10. Braak B, Klooker TK, Wouters MM, Lei A, van den Wijngaard RM, Boeckxstaens GE. Randomised clinical trial: the effects of amitriptyline on drinking capacity and symptoms in patients with functional dyspepsia, a double-blind placebo-controlled study. Aliment Pharmacol Ther. 2011;34(6):638-48.

11. Fraser A, Delaney B, Moayyedi P. Symptom-based outcome measures for dyspepsia and GERD trials: a systematic review. Am J Gastroenterol. 2005;100(2):442-52.

12. Streiner DL. Health measurement scales: a practical guide to their development and use. Oxford University Press. 2003.

13. Alrubaiy L, Hutchings HA, Williams JG. Assessing patient reported outcome measures: a practical guide for gastroenterologists. United European Gastroenterol J. 2014;2(6):463-70.

14. Terwee CB, Bot SD, de Boer MR, van der Windt DA, Knol DL, Dekker J, et al. Quality criteria were proposed for measurement properties of health status questionnaires. J Clin Epidemiol. 2007; 60(1):34-42.

15. Talley NJ, Fullerton S, Junghard O, Wiklund I. Quality of life in patients with endoscopy-negative heartburn: reliability and sensitivity of disease-specific instruments. Am J Gastroenterol. 2001; 96(7):1998-2004.

16. Berglund M, Thelander S, Salaspuro M, Franck J, Andreasson S, Ojehagen A. Treatment of alcohol abuse: an evidence-based review. Alcoholism Clin Exp Res. 2003;27(10):1645-56.

17. Shaw M, Talley NJ, Adlis S, Beebe T, Tomshine P, Healey M. Development of a digestive health status instrument: tests of scaling assumptions, structure and reliability in a primary care population. Aliment Pharmacol Ther. 1998;12(11):1067-78.

18. Shaw MJ, Beebe TJ, Adlis SA, Talley NJ. Reliability and validity of the digestive health status instrument in samples of community, primary care, and gastroenterology patients. Aliment Pharmacol Ther. 2001;15(7):981-7.

19. Tack J, Carbone F, Holvoet L, Vanheel H, Vanuytsel T, Vandenberghe A. The use of pictograms improves symptom evaluation by patients with functional dyspepsia. Aliment Pharmacol Ther. 2014;40(5):523-30.

20. Talley NJ, Verlinden M, Jones M. Quality of life in functional dyspepsia: responsiveness of the Nepean Dyspepsia Index and development of a new 10-item short form. Aliment Pharmacol Ther. 2001; 15(2):207-16.

21. Rabeneck L, Cook KF, Wristers K, Souchek J, Menke T, Wray NP. SODA (severity of dyspepsia assessment): a new effective outcome measure for dyspepsia-related health. J Clin Epidemiol. 2001; 54(8):755-65. 
22. Moayyedi P, Duffett S, Braunholtz D, Mason S, Richards ID, Dowell AC, et al. The Leeds Dyspepsia Questionnaire: a valid tool for measuring the presence and severity of dyspepsia. Aliment Pharmacol Ther. 1998;12(12):1257-62.

23. el-Omar EM, Banerjee S, Wirz A, McColl KE. The Glasgow Dyspepsia Severity Score--a tool for the global measurement of dyspepsia. Eur J Gastroenterol Hepatol. 1996;8(10):967-71.

24. Talley NJ, Haque M, Wyeth JW, Stace NH, Tytgat GN, Stanghellini V, et al. Development of a new dyspepsia impact scale: the Nepean Dyspepsia Index. Aliment Pharmacol Ther. 1999;13(2):225-35.

25. Leidy NK, Farup C, Rentz AM, Ganoczy D, Koch KL. Patient-based assessment in dyspepsia: development and validation of Dyspepsia Symptom Severity Index (DSSI). Dig Dis Sci. 2000; 45(6):1172-9.

26. Crowell MD, Umar SB, Lacy BE, Jones MP, DiBaise JK, Talley NJ. Multi-dimensional gastrointestinal symptom severity index: validation of a brief GI symptom assessment tool. Dig Dis Scie. 2015;60(8): 2270-9.

27. Goldman J, Conrad DF, Ley C, Halperin D, de la Luz Sanchez M, Villacorta R, et al. Validation of Spanish language dyspepsia questionnaire. Dig Dis Sci. 2002;47(3):624-40.

28. Garratt AM, Ruta DA, Russell I, Macleod K, Brunt P, McKinlay A, et al. Developing a condition-specific measure of health for patients with dyspepsia and ulcer-related symptoms. J Clin Epidemiol. 1996;49(5):565-71.

29. Irvine EJ, Tack J, Crowell MD, Gwee KA, Ke M, Schmulson MJ, et al. Design of treatment trials for functional gastrointestinal disorders. Gastroenterology. 2016;150(6):1469-80 e1.

30. Carbone F, Vandenberghe A, Holvoet L, Vanuytsel T, Van Oudenhove L, Jones M, et al. Validation of the Leuven Postprandial Distress Scale, a questionnaire for symptom assessment in the functional dyspepsia/postprandial distress syndrome. Aliment Pharmacol Ther. 2016;44(9):989-1001.

31. Ang D, Talley NJ, Simren M, Janssen P, Boeckxstaens G, Tack J. Review article: endpoints used in functional dyspepsia drug therapy trials. Aliment Pharmacol Ther. 2011;33(6):634-49.

32. Fransen GA, Janssen MJ, Muris JW, Mesters I, Knottnerus JA. Measuring the severity of upper gastrointestinal complaints: does GP assessment correspond with patients' self-assessment? Fam Pract. 2007;24(3):252-8.

33. Madsen LG, Hansen JM, Gronvold M, Bytzer P. The validity of a symptom diary in ratings of dyspepsia measured against a detailed interview: do patients and clinicians agree in their assessment of symptoms? Aliment Pharmacol Ther. 2007;26(6):905-12.

34. Sander GB, Mazzoleni LE, Francesconi CF, Wortmann AC, Ott EA, Theil A, et al. Development and validation of a cross-cultural questionnaire to evaluate nonulcer dyspepsia: the Porto Alegre Dyspeptic Symptoms Questionnaire (PADYQ). Dig Dis Sci. 2004;49(11-12):1822-9.

35. Adam B, Liebregts T, Saadat-Gilani K, Vinson B, Holtmann G. Validation of the gastrointestinal symptom score for the assessment of symptoms in patients with functional dyspepsia. Aliment Pharmacol Ther. 2005;22(4):357-63.

36. Administration FaD. Gastroparesis: clinical evaluation of drugs for treatment; Guidance for Industry. 2015.

37. Taylor F, Higgins S, Carson RT, Eremenco S, Foley C, Lacy BE, et al. Development of a symptomfocused patient-reported outcome measure for functional dyspepsia: The Functional Dyspepsia Symptom Diary (FDSD). Am J Gastroenterol. 2018;113(1):39-48.

38. Bradburn NM, Rips $\amalg$, Shevell SK. Answering autobiographical questions: the impact of memory and inference on surveys. Science. 1987;236(4798):157-61.

39. Feinle-Bisset C, Horowitz M. Dietary factors in functional dyspepsia. Neurogastroenterol Motil. 2006;18(8):608-18.

40. Aro P, Talley NJ, Johansson SE, Agreus L, Ronkainen J. Anxiety is linked to new-onset dyspepsia in the Swedish population: A 10-year follow-up study. Gastroenterology. 2015;148(5):928-37.

41. Mak AD, Wu JC, Chan Y, Chan FK, Sung JJ, Lee S. Dyspepsia is strongly associated with major depression and generalised anxiety disorder - a community study. Aliment Pharmacol Ther. 2012; 36(8):800-10.

42. Stone AA, Shiffman S, Schwartz JE, Broderick JE, Hufford MR. Patient compliance with paper and electronic diaries. Control Clin Trials. 2003;24(2):182-99. 
43. Tack J, Ly HG, Carbone F, Vanheel H, Vanuytsel T, Holvoet L, et al. Efficacy of mirtazapine in patients with functional dyspepsia and weight loss. Clin Gastroenterol Hepatol. 2016;14(3):385-92 e4.

44. Van Kerkhoven LL, RJ. Aparicio, N. Effect of the antidepressant venlafaxine in functional dyspepsia: a randomized, double-blind, placebo-controlled trial. Clin Gastroenterol Hepatol. 2008;6(7):746-52.

45. Matsueda K, Hongo M, Tack J, Saito Y, Kato H. A placebo-controlled trial of acotiamide for mealrelated symptoms of functional dyspepsia. Gut. 2012;61(6):821-8.

46. Carbone F, Holvoet L, Tack J. Rome III functional dyspepsia subdivision in PDS and EPS: recognizing postprandial symptoms reduces overlap. Neurogastroenterol Motil. 2015;27(8):1069-74.

47. Fang YJ, Liou JM, Chen CC, Lee JY, Hsu YC, Chen MJ, et al. Distinct aetiopathogenesis in subgroups of functional dyspepsia according to the Rome III criteria. Gut. 2015;64(10):1517-28.

48. Vakil N, Halling K, Ohlsson L, Wernersson B. Symptom overlap between postprandial distress and epigastric pain syndromes of the Rome III dyspepsia classification. Am J Gastroenterol. 2013; 108(5):767-74.

49. Carbone F, Holvoet L, Vanuytsel T, Tack J. Rome III functional dyspepsia symptoms classification: Severity vs frequency. Neurogastroenterol Motil. 2017.

50. Vanheel H, Carbone F, Valvekens L, Simren M, Tornblom H, Vanuytsel T, et al. Pathophysiological abnormalities in functional dyspepsia subgroups according to the Rome III Criteria. Am J Gastroenterol. 2017;112(1):132-40.

51. Di Stefano M, Miceli E, Tana P, Mengoli C, Bergonzi M, Pagani E, et al. Fasting and postprandial gastric sensorimotor activity in functional dyspepsia: postprandial distress vs. epigastric pain syndrome. Am J Gastroenterol. 2014;109(10):1631-9.

52. Talley NJ. Editorial: Moving away from focussing on gastric pathophysiology in functional dyspepsia: new insights and therapeutic implications. Am J Gastroenterol. 2017;112(1):141-4.

53. Lee KJ, Tack J. Duodenal implications in the pathophysiology of functional dyspepsia. J Neurogastroenterol Motil. 2010;16(3):251-7.

54. Walker MM, Aggarwal KR, Shim LS, Bassan M, Kalantar JS, Weltman MD, et al. Duodenal eosinophilia and early satiety in functional dyspepsia: confirmation of a positive association in an Australian cohort. J Gastroenterol Hepatol. 2014;29(3):474-9.

55. Liebregts T, Adam B, Bredack C, Gururatsakul M, Pilkington KR, Brierley SM, et al. Small bowel homing $\mathrm{T}$ cells are associated with symptoms and delayed gastric emptying in functional dyspepsia. Am J Gastroenterol. 2011;106(6):1089-98.

56. Talley NJ, Verlinden $M$, Jones $M$. Validity of a new quality of life scale for functional dyspepsia: a United States multicenter trial of the Nepean Dyspepsia Index. Am J Gastroenterol. 1999;94(9): 2390-7.

57. Jones M, Talley NJ. Minimum clinically important difference for the Nepean Dyspepsia Index, a validated quality of life scale for functional dyspepsia. Am J Gastroenterol. 2009;104(6):1483-8.

58. Khanna P, Agarwal N, Khanna D, Hays RD, Chang L, Bolus R, et al. Development of an online library of patient-reported outcome measures in gastroenterology: the GI-PRO database. Am J Gastroenterol. 2014;109(2):234-48.

59. Spiegel BM, Hays RD, Bolus R, Melmed GY, Chang L, Whitman C, et al. Development of the NIH Patient-Reported Outcomes Measurement Information System (PROMIS) gastrointestinal symptom scales. Am J Gastroenterol. 2014;109(11):1804-14.

60. Almario CV, Chey WD, Khanna D, Mosadeghi S, Ahmed S, Afghani E, et al. Impact of national institutes of health gastrointestinal PROMIS measures in clinical practice: results of a multicenter controlled trial. Am J Gastroenterol. 2016;111(11):1546-56.

61. Gracie DJ, Ford AC. Editorial: Using patient-reported outcome measures in gastroenterology: PROMISed land or road to nowhere? Am J Gastroenterol. 2016;111(11):1557-8.

62. Myin-Germeys I, Oorschot M, Collip D, Lataster J, Delespaul P, van Os J. Experience sampling research in psychopathology: opening the black box of daily life. Psychol Med. 2009;39(9):1533-47.

63. Stone AA, Broderick JE, Schwartz JE, Shiffman S, Litcher-Kelly L, Calvanese P. Intensive momentary reporting of pain with an electronic diary: reactivity, compliance, and patient satisfaction. Pain. 2003;104(1-2):343-51. 
64. Mujagic Z, Leue C, Vork L, Lousberg R, Jonkers DM, Keszthelyi D, et al. The Experience Sampling Method--a new digital tool for momentary symptom assessment in IBS: an exploratory study. Neurogastroenterol Motil. 2015;27(9):1295-302.

65. Weinland SR, Morris CB, Hu Y, Leserman J, Bangdiwala SI, Drossman DA. Characterization of episodes of irritable bowel syndrome using ecological momentary assessment. Am J Gastroenterol. 2011;106(10):1813-20. 


\section{Supporting information}

\section{Unidimensional disease-specific outcome measures}

\section{- Dyspepsia Symptom Severity Index}

The Dyspepsia Symptom Severity Index (DSSI) consists of 20 questions quantifying the severity of dyspeptic symptoms on a 5-point Likert scale with a recall period of two weeks. ${ }^{1}$ Subscale scores can be assessed for reflux-, ulcer- and dysmotility like symptoms. Psychometric assessment of the DSSI was performed in 48 dyspeptic patients and 24 healthy controls and demonstrated good reliability (i.e. test-retest reliability: ICC $0.90-0.93$ for total scores and subscales; internal consistency: Cronbach's $\alpha$ 0.84-0.89 for subscales and 0.76-0.80 for the total score). In addition, content validity was confirmed by experts (content validity index of $76 \%$ ), moderate to good correlations were found between DSSI and patients' symptom diary (i.e. construct validity: $\mathrm{R}=0.36-0.74, \mathrm{p}<0.05$ ), and DSSI scores could differentiate between FD patients and matched healthy controls $(p<0.01) .{ }^{1}$ Although the DSSI contains all FD core and additional symptoms, lack of information about responsiveness limits its use in clinical trials.

\section{- Global Overall Symptom Scale}

The Global Overall Symptom Scale (GOS) is a self-administered questionnaire reporting the severity of ten upper gastrointestinal symptoms using a 7-point Likert scale with a recall period of either two days (GOS2) or 28 days (GOS28). ${ }^{2}$ Psychometric validation of the GOS was tested in two large randomized controlled trials evaluating treatment efficacy of acid suppressive medication in dyspeptic patients. These trials demonstrated good test-retest reliability (ICC 0.42 and 0.62 , GOS28 and GOS2 respectively). Construct validity was assessed by correlating GOS to other symptom scores (e.g. GSRS) demonstrating good correlations ( $R=0.41-0.80)$. Moreover, strong negative correlations where found with the QolRAD which is a quality of life questionnaire (i.e. higher symptom scores are associated with lower quality of life; $\mathrm{R}=$ -0.62 till -0.69 ). In addition, responsiveness was demonstrated by correlating changes in GOS scores with changes in symptom severity in which responders reported larger symptom changes when compared to non-responders. ${ }^{2}$ Overall effect sizes were 3.0 (GOS2) and 1.1 (GOS28), whereas effect sizes for responders were higher compared to non-responders (GOS2: 3.8 vs. 1.2; GOS28: 3.3 vs. 0.7). Lack of assessment of epigastric burning is a limitation as this is considered a core symptom of FD. ${ }^{3}$ 


\section{- Leeds Dyspepsia Questionnaire}

The Leeds Dyspepsia Questionnaire (LDQ) evaluates the frequency and severity of eight upper gastrointestinal symptoms with a six months recall period. ${ }^{4}$ Psychometric validation of the LDQ was performed in a primary and secondary care population with dyspeptic symptoms. ${ }^{4}$ Test-retest reliability (weighted kappa $(K w)$ of 0.83 ), interrater reliability $(K w=0.90)$ and internal consistency (Cronbach's $\alpha 0.69)$ were excellent. Comparison of LDQ scores with severity assessment by clinicians was used to assess validity, and demonstrated good agreement $(K w=0.49-0.58)$. Although responsiveness was assessed in only a small subgroup of 12 patients with esophagitis or peptic ulcer disease, a significant reduction of symptoms was observed after treatment with either proton pump inhibitors and/or Helicobacter Pylori eradication therapy (i.e. score reduction from 22.5 to 4.5 , Wilcoxon signed rank test: $\mathrm{p}<0.01$ ). Psychometric validation has also been performed for Mandarin, Malay and Malaysian English versions of the LDQ. ${ }^{5,6}$ Disadvantages of the LDQ are use of a complex scoring system, investigator-administration, and long recall period.

To overcome these two last shortcomings of the LDQ, the patient-administered short-form LDQ (SF-LDQ) was developed which contained four questions measuring frequency and severity of indigestion, heartburn, regurgitation and nausea in the preceding two months. ${ }^{7}$ Psychometric assessment was performed in 592 primary and secondary care patients without specific selection of patients with dyspeptic symptoms. High internal consistency (Cronbach's $\alpha$ 0.90) and test-retest reliability (Pearson's correlation coefficient of 0.93) were found. Concurrent validity was assessed by comparing SF-LDQ with general practitioners' opinion of whether the patient had dyspepsia using logistic regression analysis and demonstrated strong associations $(p<0.01)$. In addition, the SF-LDQ could discriminate between primary and secondary care populations with different prevalence of dyspepsia (i.e. known groups validity; $p<0.01$ ) and was responsive to change in 37 patients with esophagitis or peptic ulcer treated with acid suppressive medication and/or $\mathrm{H}$. Pylori eradication therapy (standardized response mean: 1.1). Psychometric validation has also been performed for the Italian and Kinyarwanda version of the SF-LDQ. ${ }^{8,9}$ Major limitation of the SF-LDQ is lack of assessment of FD core symptoms such as postprandial fullness and early satiation. ${ }^{3}$

\section{- Gastrointestinal Symptom Rating Scale}

The Gastrointestinal Symptom Rating Scale (GSRS) was originally developed as an interview-based outcome measure for patients with irritable bowel syndrome and peptic ulcer disease but was modified to a self-administered scale. ${ }^{10-13}$ The instrument consists of 15 items addressing five symptom clusters (i.e. abdominal pain, gastroesophageal reflux, indigestion, diarrhea and constipation) using a 7-point Likert scale. Different recall periods of one week and one month have been used. ${ }^{10,11}$ 
Psychometric characteristics of the GSRS were evaluated in patients with endoscopynegative heartburn and patients with dyspeptic symptoms. ${ }^{11,12}$ Although good interrater reliability $(K w=0.86-1.00)$ and test-retest reliability were observed in endoscopy-negative heartburn patients (ICC 0.53-0.69), test-retest reliability and internal consistency differed between GSRS domains in patients with dyspeptic symptoms in several languages (reliability: ICC 0.36-0.75; internal consistency: Cronbach' $\alpha$ 0.43-0.87). ${ }^{11-13}$ Especially the abdominal pain domain showed only moderate reliability and consistency values, which probably reflects the fluctuating nature of this symptom and fact that this domain only contains two items. ${ }^{12}$ Construct validity of the GSRS was demonstrated by comparison with additional outcome measures (e.g. SF-36; majority of GSRS domains were negatively correlated with categories of SF-36). In addition, all domains of the GSRS were able to differentiate between groups of patients whose health status differed according to expert opinion (i.e. GSRS scores increased in patients with increasing symptom frequency and/or severity) although results are not shown (i.e. known groups validity). The instrument was considered responsive to change in patients with endoscopy-negative heartburn treated with proton pump inhibitors (i.e. effect size: -0.16 till -1.74 for different GSRS domains; standardized response mean: -0.15 till -1.43 for different GSRS domains), but it has never been assessed in dyspeptic patients. ${ }^{11-13}$ Moreover, the GSRS is not specific for dyspepsia and does not evaluate all FD core symptoms.

\section{- Hong Kong Dyspepsia Index}

The Hong Kong Dyspepsia Index uses 12 items to assess severity of gastrointestinal symptoms using a 5-point Likert scale. ${ }^{14}$ Psychometric evaluation of the Hong Kong Dyspepsia Index was performed in 65 Chinese patients and demonstrated good reliability (i.e. test-retest reliability: ICC 0.89 ; internal consistency: Cronbach's $\alpha 0.82$ ). Significant correlations were found between the Hong Kong Dyspepsia Index and several subscales of the SF-36 supporting construct validity $(r=-0.27$ till -0.34$)$, although scores were not correlated with the physical functioning and vitality subscales of the SF-36 ( $r=0.09$ and -0.12 respectively). Moreover, a significant correlation was found between the symptom score three weeks after treatment with cisapride and whether the patients reported symptomatic improvement (Kendall's tau $0.21, p=0.02$ ). Disadvantages of the Hong Kong Dyspepsia Index are however the unknown recall period and lack of several FD core symptoms (e.g. early satiety).

\section{- Bologna severity index}

The Bologna severity index was developed as an investigator-administered 8-item instrument for assessment of symptom severity and frequency on a 5-point Likert scale. ${ }^{15}$ Seventy-three patients with dyspeptic symptoms according to Rome criteria and 75 healthy controls fulfilled this questionnaire. Reproducibility was assessed using 
intra-observer and inter-observer variability with moderate correlation coefficients of 0.62 and 0.60 respectively. Validity was demonstrated by significant differences in scores between healthy controls and dyspeptic patients (i.e. 1.4 for healthy controls and 4.8 in dyspeptic patients, $\mathrm{p}<0.01$ ). Moreover, symptom scores were reduced after treatment (i.e. from 39.2 to $15.2, p<0.01$ ). Limitations of the questionnaire are the investigator interview approach, undefined recall period, small population of patients with functional dyspepsia $(n=15)$ and lack of several FD core symptoms.

\section{- Porto Alegre Dyspeptic Symptoms Questionnaire}

The Porto Alegre Dyspeptic Symptoms Questionnaire (PADYQ) was developed as an investigator administered 11-item instrument which evaluates the intensity, frequency and duration of five symptoms (i.e. pain in the upper abdomen, nausea, vomiting, upper abdominal bloating and early satiety). ${ }^{16}$ Psychometric validation was performed in 31 FD patients according to the Rome I criteria and 31 healthy controls. Factorial analysis identified three domains: (1) Bloating/Satiety domain, (2) Vomiting/Nausea domain, and (3) Pain domain. Test-retest reliability was good both by the same and different interviewers (ICC 0.86 and 0.87 , respectively). Internal consistency was adequate (i.e. Cronbach's $\alpha$ 0.82). Content validity was confirmed by two experienced gastroenterologists and the PADYQ could discriminate between FD patients and healthy controls (mean score 22.2 for dyspeptic patients and 0.9 for healthy controls, $p<0.01$ ). Criterion validity was confirmed by correlating outcomes from the PADYQ with a quality-of-life questionnaire demonstrating a significant although moderate correlation $(\mathrm{R}=-0.29, \mathrm{p}=0.02)$. In addition, responsiveness was demonstrated in dyspeptic patients treated with either acid suppressive medication or H. Pylori eradication therapy (i.e. decrease in score from 23.2 to $16.0, p<0.01$ ). Lack of inclusion of several FD core symptoms including postprandial fullness and epigastric burning remains the main disadvantage of the PADYQ.

\section{- Gastrointestinal Symptom Score}

The Gastrointestinal Symptom Score, an investigator-administered 10-item questionnaire, was developed by patient focus groups to assess the severity of upper gastrointestinal symptoms on a 5-point Likert scale. ${ }^{17}$ Psychometric assessment of this outcome measure was performed in 95 functional dyspepsia patients according to Rome II criteria and showed good test-retest reliability ( $r=0.84-0.90)$, convergent validity ( $R=0.67-0.76$, by comparing subscales with the Nepean Dyspepsia Index), and responsiveness (i.e. significant larger reduction in symptom scores in responders compared to non-responders after treatment with STW5). ${ }^{17}$ Moreover, symptom scores were higher for dyspeptic patients compared to healthy controls, and significant correlations were found between symptom scores and external assessment of whether participants were patients or healthy controls $(r=0.44-0.61)$. Limitations of 
the Gastrointestinal Symptom Score are the unknown recall period and lack of assessment of FD core symptoms.

\section{- Gastrointestinal Symptoms Severity Index}

The Gastrointestinal Symptoms Severity Index (GISSI) was recently described by the group of Talley. ${ }^{18}$ The GISSI is a multi-dimensional, self-report outcome measure to assess severity, frequency and botheration of 32 upper and lower gastrointestinal symptoms and seven items related to pelvic floor and urogynaecologic symptoms. Nine hundred thirty-four patients referred for gastrointestinal motility testing and 200 healthy controls were included. Factorial analysis revealed six symptom clusters based on 23 items (and four additional items specific for females): constipation/difficult defecation, abdominal pain/discomfort, dyspepsia, diarrhea/anal incontinence, GERD/chest symptoms, and nausea/vomiting. Internal consistency (Cronbach's $\alpha 0.60$ 0.89 ), test-retest reliability (ICC $0.70-0.93$ ) and content validity were good. Although known groups validity was considered adequate, the GISSI did not differ between patients with and without the primary diagnosis of dyspepsia at intake which might be due to the fact that dyspepsia was rarely the primary indication for study. Instead, patients were more often referred for early satiety, bloating and fullness. The GISSI seems a promising tool to provide information about overlapping symptom clusters, whereas the use in specific FD trials is currently limited due to lack of validation in this target population, no available information about responsiveness, and inclusion of not all FD core symptoms.

\section{- Digestive Health Status Instrument}

The Digestive Health Status Instrument (DHSI) was originally developed as a 54-item instrument but was reduced to a 34-item questionnaire after extensive analyses. ${ }^{19}$ Content validity was considered good and a recall period of four weeks was used. Factorial analysis revealed five subscales: reflux, dysmotility, pain, and a two-domain bowel dysfunction complex (i.e. diarrhea and constipation). Psychometric validation has been performed in 690 primary care patients presenting with abdominal pain, discomfort or heartburn. Internal consistency was good (i.e. Cronbach's $\alpha$ 0.63-0.90) and criterion validity demonstrated the strongest correlations between pain score of the DHSI and pain score of the SF-36 $(R=-0.50)$. Overall, correlations ranged between $r=-0.02$ till -0.50 for DHSI domains and subcategories of SF-36. Additional psychometric validation was performed in a randomized, double-blind, placebocontrolled trial of 45 GERD patients treated with either placebo or omeprazole which demonstrated good test-retest reliability and responsiveness. ${ }^{20}$ However, test-retest reliability and responsiveness have not been evaluated in dyspeptic patients. Therefore, use of the DHSI is limited due to lack of information about responsiveness in dyspeptic patients, and relatively long recall period. 


\section{- Leuven Postprandial Distress Scale}

Recently, the Leuven Postprandial Distress Scale (LPDS) was developed as PROM for patients with functional dyspepsia/postprandial distress syndrome. ${ }^{21,22}$ Focus groups and cognitive interviews were performed to identify relevant symptoms in PDS patients. ${ }^{21,23}$ All PDS patients reported postprandial fullness and early satiety, thereby confirming these key items for development of a PROM for PDS patients. ${ }^{21}$ Based on these results, a provisional diary instrument was developed which contains eight items assessing severity of upper abdominal symptoms using verbal descriptors combined with "smiley faces". ${ }^{22}$ Items of the LPDS can be divided into two domains according to the Rome III criteria and performed factor analysis: (1) postprandial distress syndrome items including early satiation, postprandial fullness and abdominal bloating, and (2) epigastric pain syndrome items including epigastric pain and epigastric burning. The LPDS was developed for daily use which has the advantage of reducing recall bias.

Psychometric validity of the LPDS was performed in a double-blind randomized, multicenter, placebo-controlled study with 60 patients receiving either itopride or placebo. Twenty-nine patients (48\%) had PDS, whereas $52 \%$ had overlapping PDS and EPS. Internal consistency (i.e. Cronbach's $\alpha$ 0.72-0.86) and test-retest reliability $(r=0.85-0.86)$ were good for both FD subtypes. To assess convergent validity, results of the LPDS domains were compared with the Nepean Dyspepsia Index (NDI), PAGI-SYM and overall symptom severity. The overall symptom severity correlated with both the PDS ( $R=0.70)$ and EPS domains ( $R=0.48)$ of the LPDS. In addition, strong correlations were found between the PDS domain of the LPDS and both the early satiation/postprandial fullness subscale of the PAGI-SYM $(R=0.66)$ and eat/drink subscale of the NDI ( $R=0.58)$ providing evidence for construct validity. Moreover, patients with more severe symptoms reported higher symptom scores compared to patients with lower symptom scores (i.e. known groups validity; PDS: Cohen's $d=2.16$; EPS: Cohen's $d=1.24)$. For use of PROMs in therapeutic trials, sensitivity to change (e.g. responsiveness) is necessary. Carbone et al. demonstrated a strong correlation between the overall treatment evaluation with longitudinal changes in the PDS domain of the LPDS $(R=-0.52, p<0.0001)$. Moreover, a minimum clinically important difference has been defined of 0.5 points on the 0-4 range of the LPDS. Therefore, it was concluded that the LPDS is a valid, reliable and responsive patient-reported outcome measure for patients with PDS-FD. ${ }^{22}$ Although supportive results were also found for the validity and reliability of the LPDS for assessment of the EPS syndrome, responsiveness was not demonstrated probably due to the more prevalent and more severe PDS symptoms. ${ }^{22}$ Therefore, additional research is necessary before use of the LPDS for assessment of EPS symptoms in clinical trials. 


\section{- Functional Dyspepsia Symptom Diary}

The Functional Dyspepsia Symptom Diary (FDSD) is the most recently developed outcome measure for assessment of dyspeptic symptoms. ${ }^{24}$ Forty-five face-to-face interviews and an expert panel meeting were organized to identify dyspeptic symptoms in FD patients and develop a preliminary construction of the FDSD. This preliminary FDSD outcome measure consisted of eight items: severity of stomach pain, upper abdominal burning, nausea, bloating, postprandial fullness, and early satiety. Moreover, the severity and bothersome influence of belching were evaluated. Responses were scored on a 11-point numeric rating scale with a recall period of 24 hours. The Total Symptom Score comprised stomach pain, upper abdominal burning, bloating, postprandial fullness and early satiety. Nausea and belching were considered supplementary items as they were not considered cardinal FD symptoms by the expert panel.

This preliminary FDSD was evaluated by 57 patients using face-to-face interviews. This PROM was considered comprehensible and all included items were relevant. Moreover, use of the FDSD on an electronic device was considered appropriate. Internal consistency was good with a Cronbach's $\alpha$ of 0.87 . In addition, known groups validity was demonstrated as the Total Symptom Score increased across known severity groups (i.e. patients with severe FD had higher symptom scores compared to patients with mild/moderate FD). This newly developed PROM is therefore considered content-valid and seems promising for the future. However, additional research is necessary to evaluate the test-retest reliability, construct validity and responsiveness before use in clinical trials.

\section{- Patient Assessment of Upper Gastrointestinal Symptom Severity Index (PAGI-SYM)}

The Patient Assessment of Upper Gastrointestinal Symptom Severity Index (PAGISYM) has been developed to measure symptom severity in patients with gastroesophageal reflux disease, dyspepsia and gastroparesis. ${ }^{25}$ The PAGI-SYM includes 20 items divided into six subscales scored on a 6-point Likert scale: (1) heartburn/regurgitation, (2) nausea/vomiting, (3) postprandial fullness/early satiety, (4) bloating, (5) upper abdominal pain, and (6) lower abdominal pain. ${ }^{25}$ Psychometric validation was performed in patients with GERD, dyspepsia and gastroparesis and demonstrated good reliability (i.e. internal consistency: Cronbach's $\alpha$ 0.74-0.90; test-retest reliability: ICC $0.61-0.81$ ). Construct validity was confirmed by moderate to good statistically significant correlations between the PAGI-SYM and subscales of the SF-36 (i.e. correlations ranged between -0.23 till -0.47 ). In addition, correlations between PAGI-SYM subscales and patient-rated global symptom severity ranged between 0.32 till 0.51 . Dyspeptic patients with symptom relief after undefined therapy reported significant better symptom scores when compared to patients without symptom relief which demonstrates responsiveness of this outcome 
measure. ${ }^{26}$ Effect sizes in dyspeptic patients ranged between 0.42-1.22 for PAGI-SYM subscales and were considered good $(>0.80)$ for fullness/early satiety, bloating and upper abdominal pain domains. Moreover, a MCID is available. One limitation of the PAGI-SYM is the inclusion of several non-FD symptoms. However, Revicki et al. suggested that selected subscales within the PAGI-SYM might be useful as endpoint in clinical trials evaluating treatment efficacy. Although it is uncertain which subscales might be most useful to evaluate treatment efficacy in dyspeptic patients, the fullness/early satiety, bloating, and upper abdominal pain subscales seemed most responsive. $^{26}$

\section{Multidimensional disease-specific outcome measures}

\section{- Nepean Dyspepsia Index}

The Nepean Dyspepsia Index (NDI) was developed as a disease-specific quality of life measure. $^{27-30}$ It contains 25-items concerning five subscales: (1) interference with activities of daily living, (2) impact of dyspepsia on work/study, (3) disturbance of eating or drinking, (4) knowledge/control of dyspepsia, and (5) effect on tension/sleep..$^{27-29}$ In addition, a separate symptom checklist measures the frequency, intensity and botheration of 15 upper gastrointestinal symptoms with a recall period of two weeks. ${ }^{27}$

\section{- Quality of life component}

Initial psychometric assessment was performed in 113 patients with gastrointestinal complaints of which $81 \%$ had dyspepsia, and in a randomly chosen population-based sample of 347 persons. ${ }^{27}$ Face and content validity were considered adequate by an international expert panel. Factor analysis identified five subscales with good internal consistency (Cronbach's $\alpha$ 0.81-0.96) and excellent test-retest reliability (ICC 0.85-0.94). ${ }^{27,29}$ Moreover, the NDI quality of life measurement could discriminate between dyspeptic patients and healthy controls (i.e. significant worse scores in dyspeptic patients compared to healthy controls) and weak to moderate correlations were found with several scales of the SF-36 (ranging between $r=-0.25$ till $r=0.39$ ). ${ }^{27,29}$ In addition, NDI subscales correlated moderately with patient global rating of quality of life ( $r=0.25-0.35)$, and symptom checklist of NDI correlated with patient global rating of symptom severity ( $r=0.39)$. A strong negative correlation was found between the symptom severity assessed by the symptom checklist and quality of life measurement $(r=-0.74){ }^{27}$ Responsiveness has been demonstrated in 589 dyspeptic patients randomized to either motilin or placebo for four weeks with moderate correlations between change in quality of life and patient self-reporting. ${ }^{28}$ Standardized response means were good for both the placebo and motilin group and ranged between 1.18 and 1.28 . In addition, a change of at least 10 points on the NDI 
total scale is considered as a clinically meaningful change (i.e. minimally clinically important difference). ${ }^{30}$ Assessment of psychometric properties has also been performed for a Chinese version. ${ }^{31}$

\section{- Symptom checklist}

Known groups validity and responsiveness have been established for the symptom checklist. ${ }^{27,28}$ A strong correlation was found between the change in NDI symptom score and patients' self-reported symptoms $(r=0.54)$. Standardized response means were good for both the placebo and motilin group (i.e. 0.98 and 1.14, respectively). However, test-retest reliability and convergent validity have not been assessed.

\section{- Short-form NDI}

In order to develop a valid and short quality of life measure for patients with functional dyspepsia, the short-form NDI was developed. ${ }^{28}$ Two items with the highest factor loadings on each of the five subscales were included, yielding a 10-item quality of life scale. The short-form NDI had good internal consistency (Cronbach's $\alpha$ 0.700.76 for subscales) and demonstrated responsiveness. ${ }^{28}$ Although strong correlations were found between results of the full NDI and short-form NDI (i.e. $r=0.71-0.85$ at baseline), comparisons were not performed between the short-form NDI and independent gastrointestinal questionnaires. Moderate correlations were found between changes in SF-NDI quality of life scores and patient's reported symptoms ( $r=0.26-0.40$; responsiveness). Psychometric validation has also been performed for a Malay and Kinyarwanda version of the SF-NDI. ${ }^{9,32}$

In conclusion, assessment of both quality of life and symptoms is a strength of the NDI as it is assumed that a change in symptoms may parallel a change in quality of life. In addition, the symptom checklist does assess all core symptoms of FD and a minimally clinically important difference is available. However, test-retest reliability and validity have not been assessed for the symptom checklist, and the short-form NDI does only measure quality of life without determining symptom severity or frequency.

\section{- Glasgow Dyspepsia Severity Score}

The Glasgow Dyspepsia Severity Score (GDSS) is a short outcome measure used for the global evaluation of symptom severity but it does not provide information about the type of symptoms. ${ }^{33}$ The questionnaire contains eight items measuring frequency of dyspeptic symptoms, effect on normal activities, time off work due to symptoms, frequency of medical consultations, number of home visits by a general practitioner, number of diagnostic tests performed for the dyspeptic symptoms, and use of both prescribed and over-the-counter medication. The GDSS has a long recall period of three or six months. ${ }^{33,34}$ Originally, the questionnaire was investigator-administered 
but Calvet et al. evaluated the use of this questionnaire in a phone interview. ${ }^{34}$ The GDSS demonstrated high reproducibility when performed by different methods (i.e. phone and clinical interview) as well as by different observers. Psychometric validation was performed in healthy controls, functional dyspepsia patients and patients with ulcer disease and demonstrated good inter- and intra-rater reliability (i.e. coefficient of variance of $8 \%$ and $2 \%$ respectively). Moreover, symptom scores could discriminate between (non)-ulcer patients and healthy controls. ${ }^{33,34}$ Moreover, responsiveness was demonstrated as symptom scores decreased after successful eradication of Helicobacter Pylori in patients with duodenal ulcer disease with reduction of symptom score from 11.4 to 1.3 . Limitations of the GDSS are administration by an investigator, the global evaluation of symptom frequency instead of assessment of individual dyspeptic symptoms, lack of measurement of FD core symptoms and the long recall period. ${ }^{35}$

\section{- Spanish Language Dyspepsia Questionnaire}

The Spanish Language Dyspepsia Questionnaire (SLDQ) addresses seven aspects of dyspepsia including evaluation of symptoms and influence on quality of life. ${ }^{36}$ The SLDQ is investigator-administered and has been evaluated in 63 participants (healthy controls and patients with self-reported dyspeptic symptoms) in Chiapas (Mexico). Reliability was considered adequate (internal consistency: Cronbach's $\alpha$ 0.93, interrater reliability: ICC 0.96), and correlations between subscales of the SLDQ and domains of the SF-36 ranged between $r=-0.13$ till -0.60 (mean: $r=-0.34$ ). Moreover, significant higher scores were found in self-reported dyspeptic patients compared to healthy controls. Responsiveness has not been assessed. Limitations of the SLDQ are the lack of validation in patients with diagnosed dyspepsia, unavailable information about responsiveness, undefined recall period and psychometric validation in only the Spanish language.

\section{- The Clinical Dyspepsia Questionnaire}

The Clinical Dyspepsia Questionnaire was developed as a self-administered outcome measure to assess dyspeptic symptoms and quality of life. ${ }^{37}$ Although no official recall period has been described, questions refer to two weeks, one month and three months. Psychometric validation has been performed in 199 patients with dyspepsia or ulcer-related problems either referred to the outpatient clinic or under treatment of general practitioners. Internal consistency (Cronbach's $\alpha$ 0.72) and test-retest reliability were acceptable (ICC 0.69). Content validity was considered adequate and significant moderate to good negative correlations were found with subscales of the SF-36 ( $R=-0.20$ till -0.66). Moreover, symptom scores were higher in referred patients compared to non-referred patients, and general practitioners' opinion of symptom 
severity was related to scores of the questionnaire. Use of this questionnaire is limited by lack of information about responsiveness and use of different recall periods.

- Severity of Dyspepsia Assessment (SODA)

The Severity of Dyspepsia Assessment (SODA) is a multidimensional outcome measure to evaluate dyspepsia-related health which was developed after extensive research. ${ }^{38-}$ ${ }^{40}$ Factor analyses revealed three dimensions of dyspepsia-related health: (1) pain intensity, (2) non-pain symptoms, and (3) satisfaction with dyspepsia-related health. ${ }^{40}$ The SODA is self-administered and uses a recall period of one week. Psychometric validation was performed in a small randomized controlled trial with 98 dyspeptic patients receiving either omeprazole or placebo. ${ }^{40}$ Internal consistency of the three dimensions was excellent with Cronbach's $\alpha$ ranging from 0.90 till 0.97 . Test-retest reliability was not assessed in dyspeptic patients, only in patients with arthritis receiving cox-2-inhibitors (ICC $0.45-0.61$ for the subscales).$^{41}$ Criterion validity was demonstrated by comparing mean change scores between dyspeptic patients with symptom improvement and patients with unchanged symptoms and demonstrated statistically significant higher mean change scores for patients with symptom improvement. $^{40}$ In addition, responsiveness was assessed in dyspeptic patients treated with either acid suppressive medication or placebo. Responsiveness for the 'pain intensity' and 'dyspepsia-related health' dimensions was high (effect sizes: 0.98 and 0.87 , respectively), whereas the effect size was small for non-pain symptom scores (i.e. 0.24). One limitation of this psychometric validation was that it was performed in mainly dyspeptic male patients questioning the generalizability of the results. Moreover, this outcome measure does not contain all FD core symptoms and uses a difficult scoring system with different answer categories. Welle et al. performed several modifications of the pain intensity scale of the SODA for assessment of dyspeptic symptoms in patients treated with NSAIDs for osteoarthritis; the recall period was reduced to 24 hours and electronic data collection was performed. $^{42}$ 


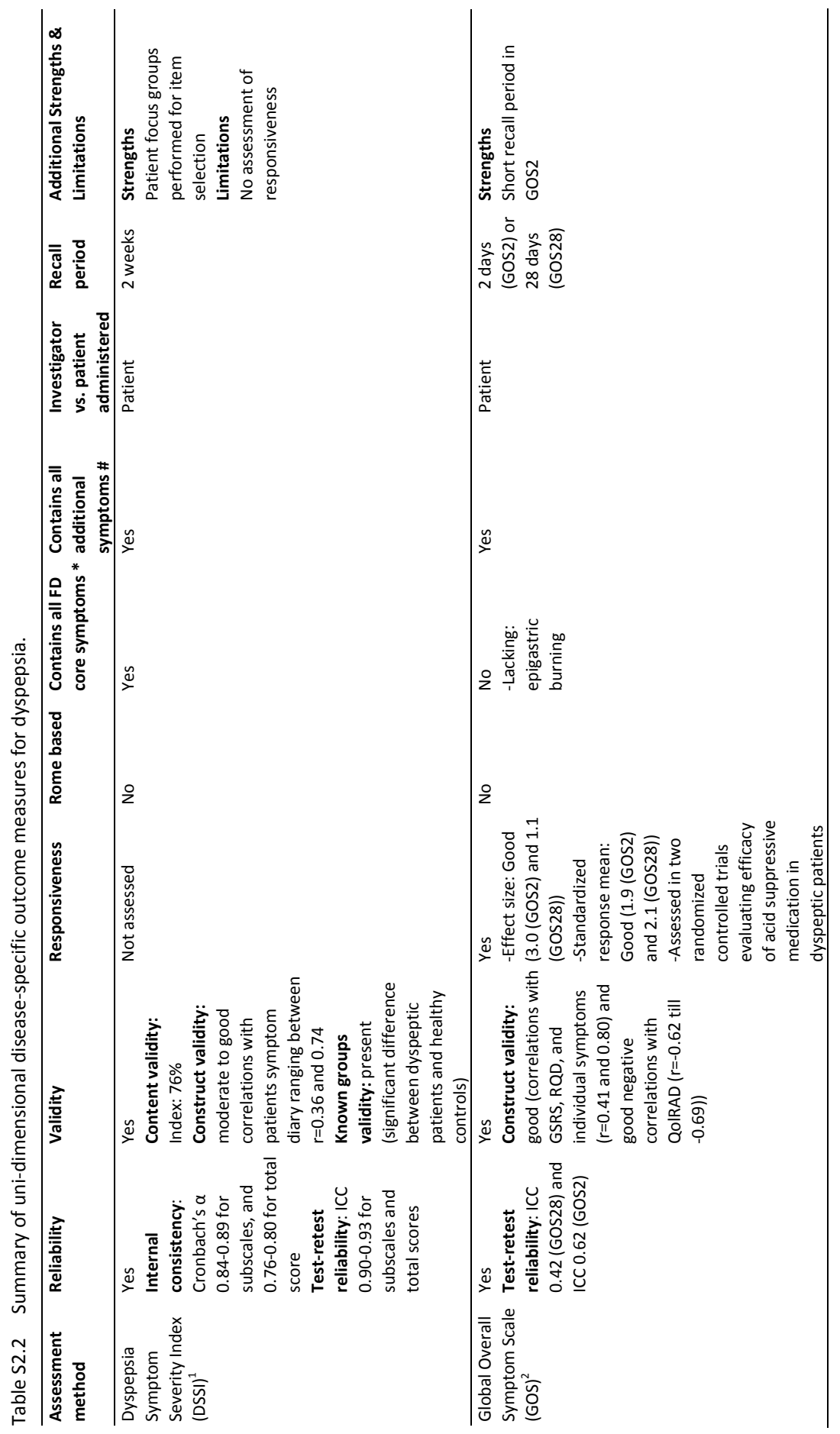




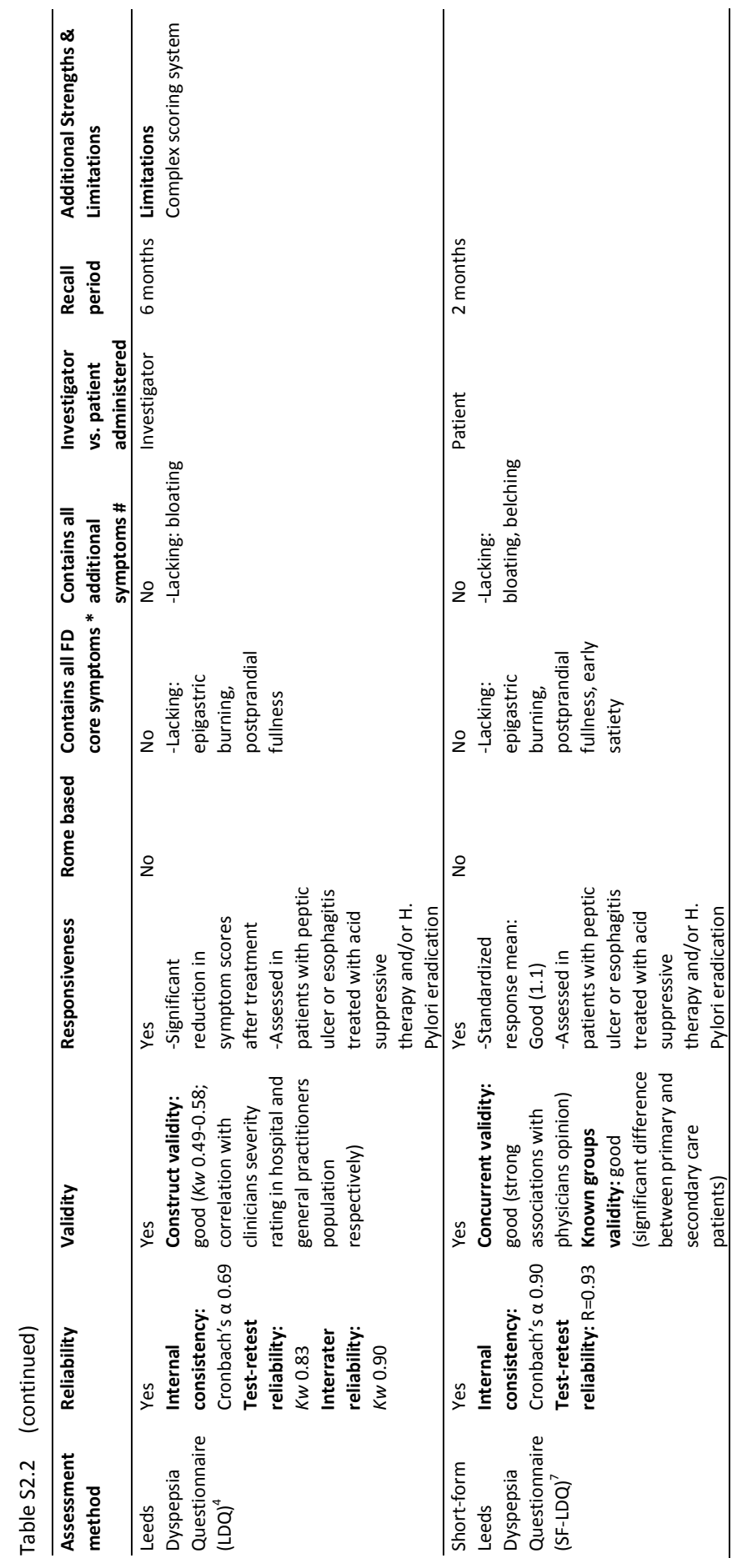




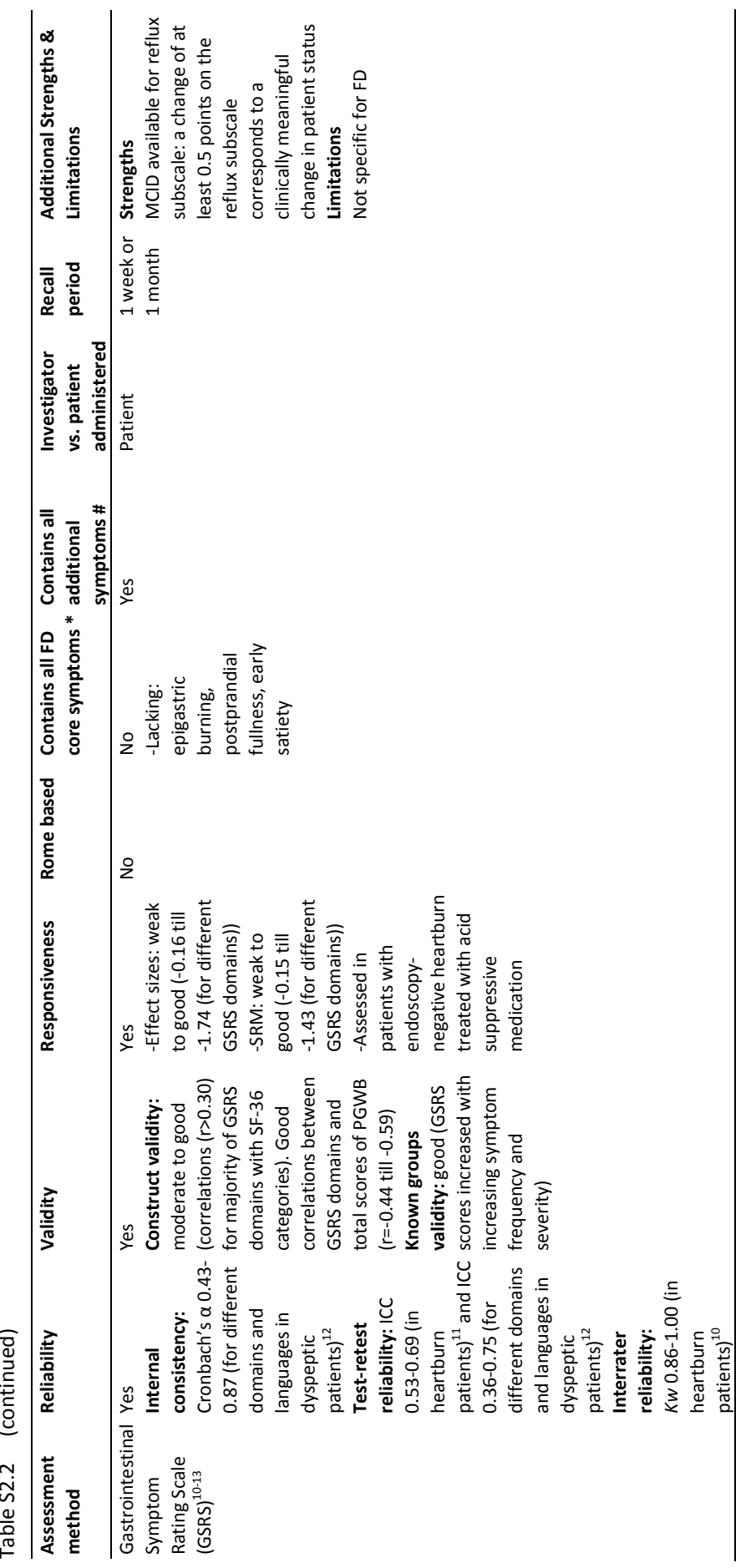




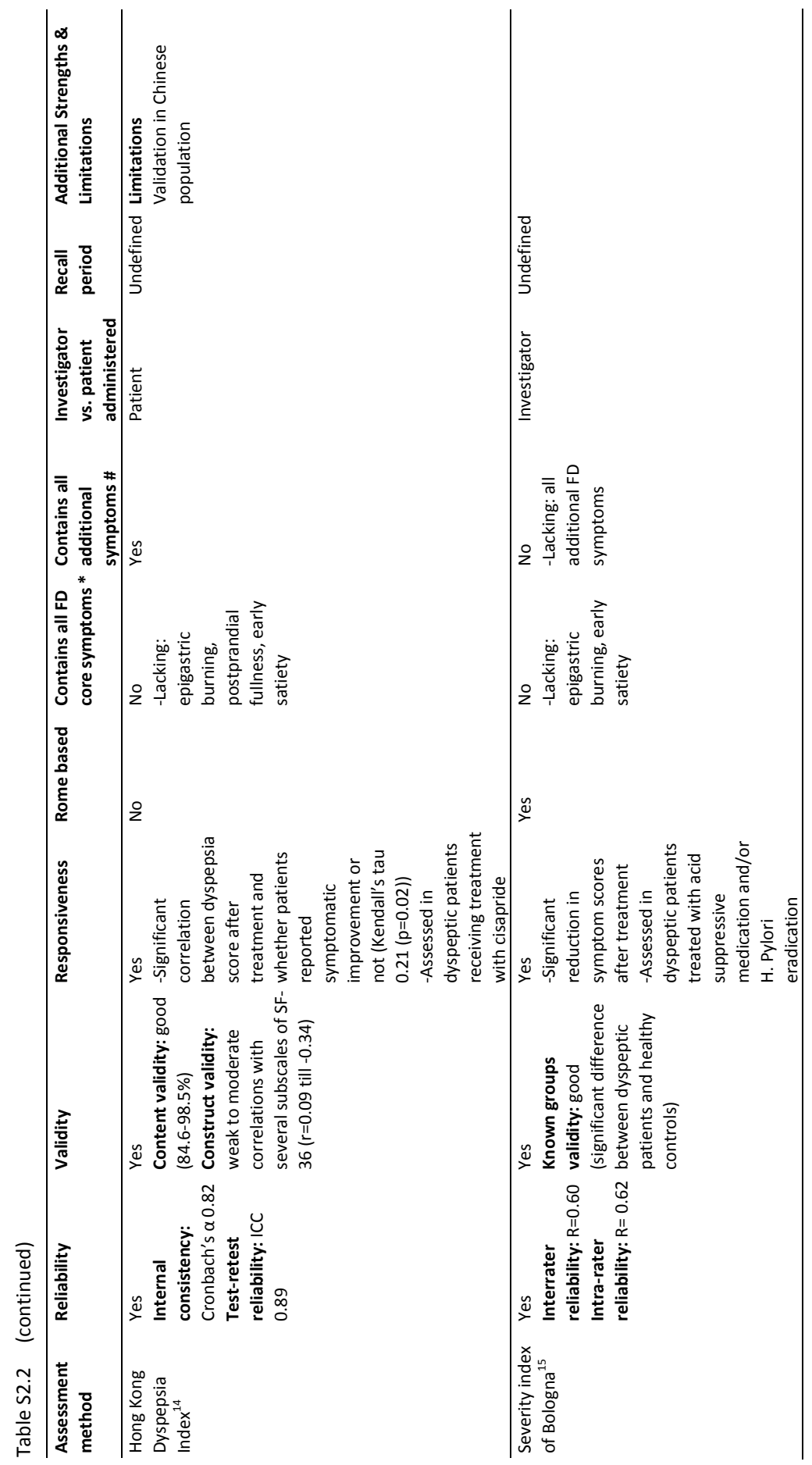




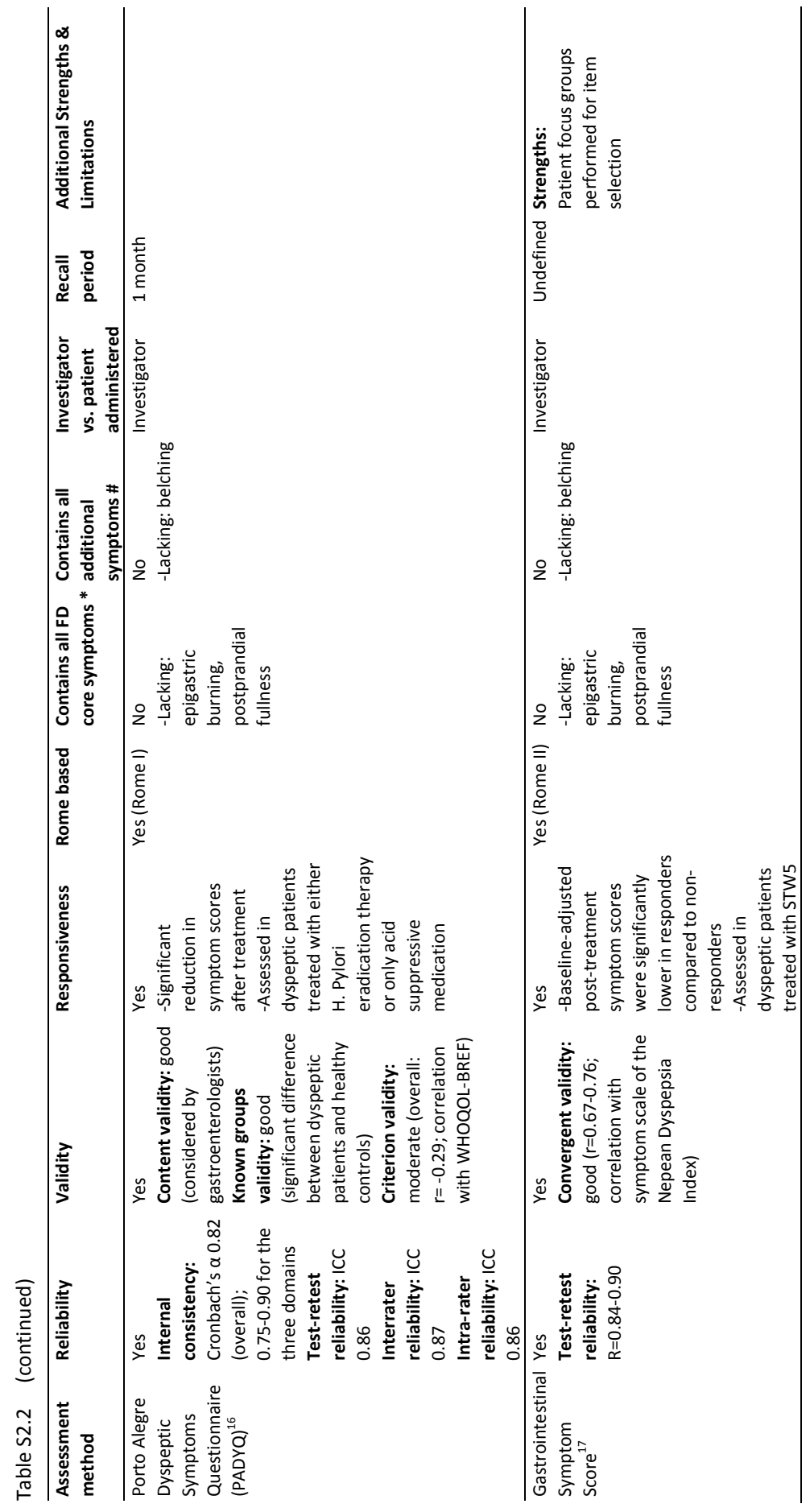




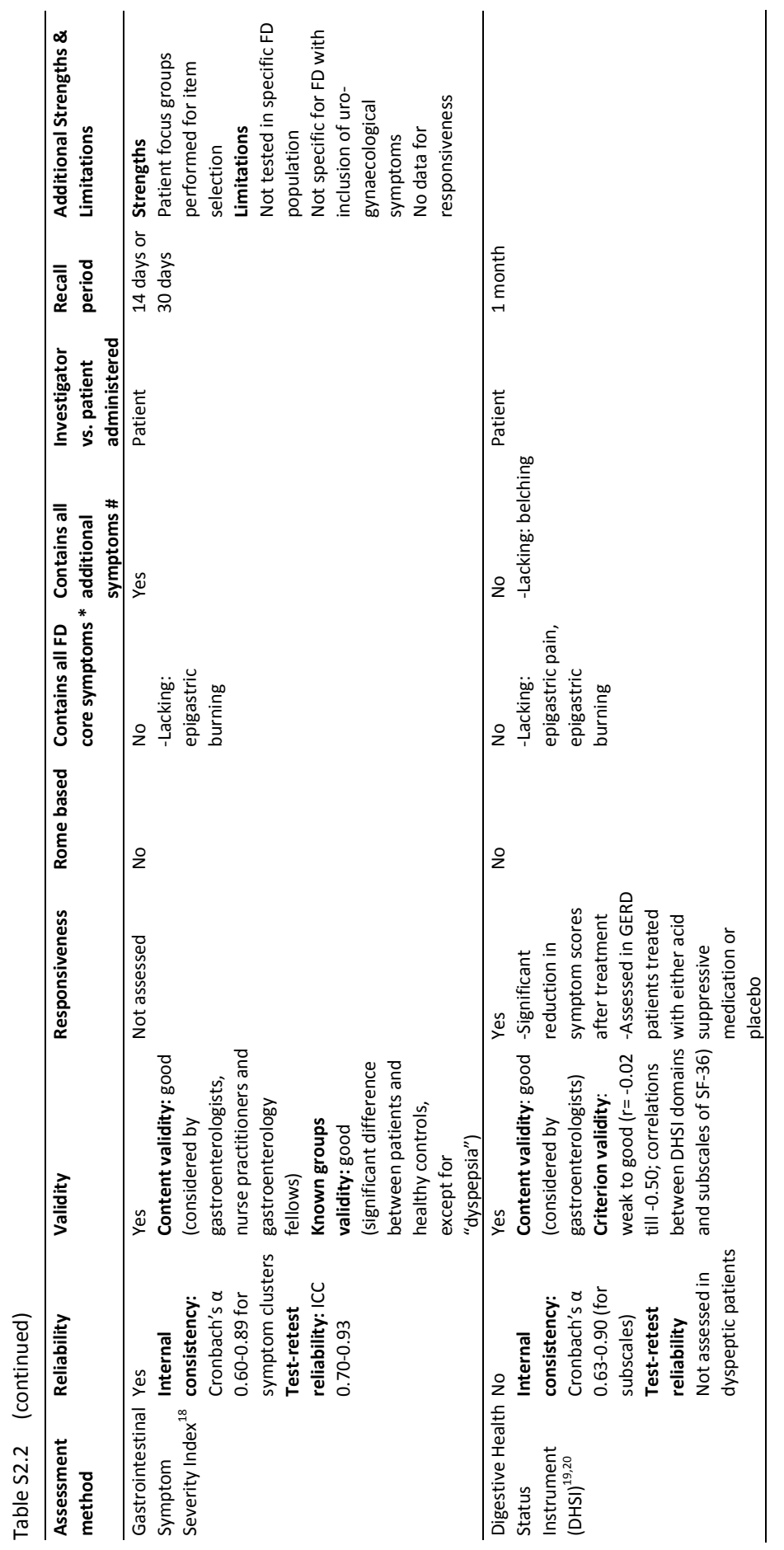




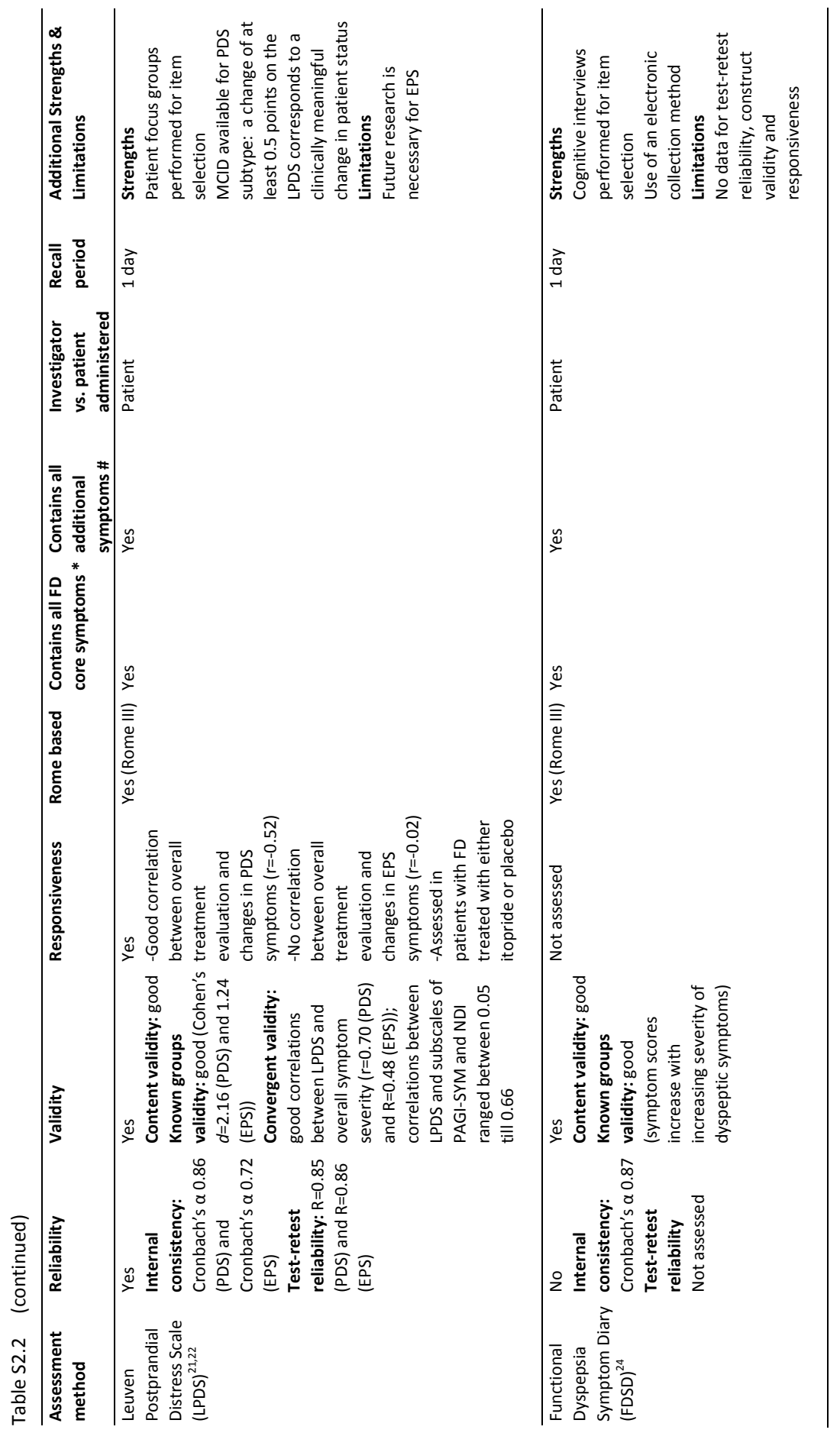




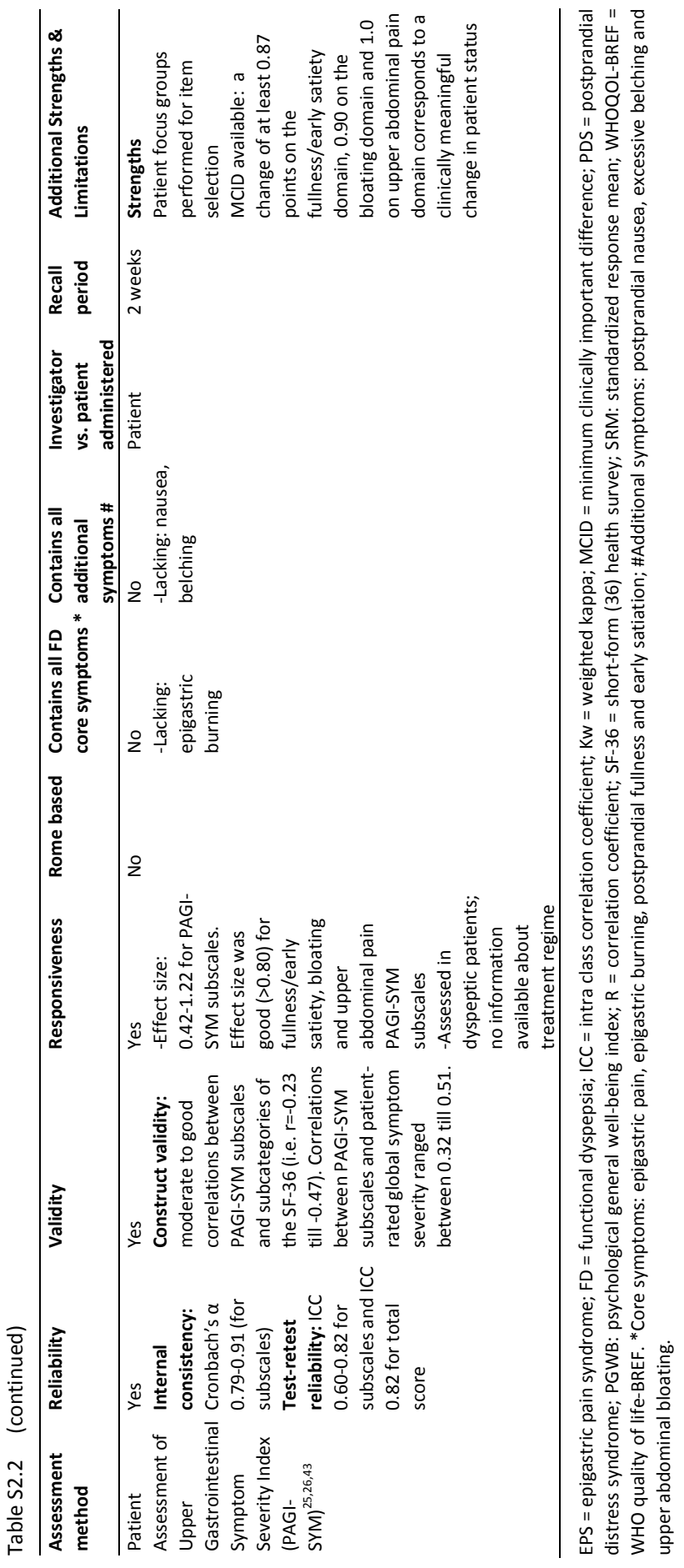




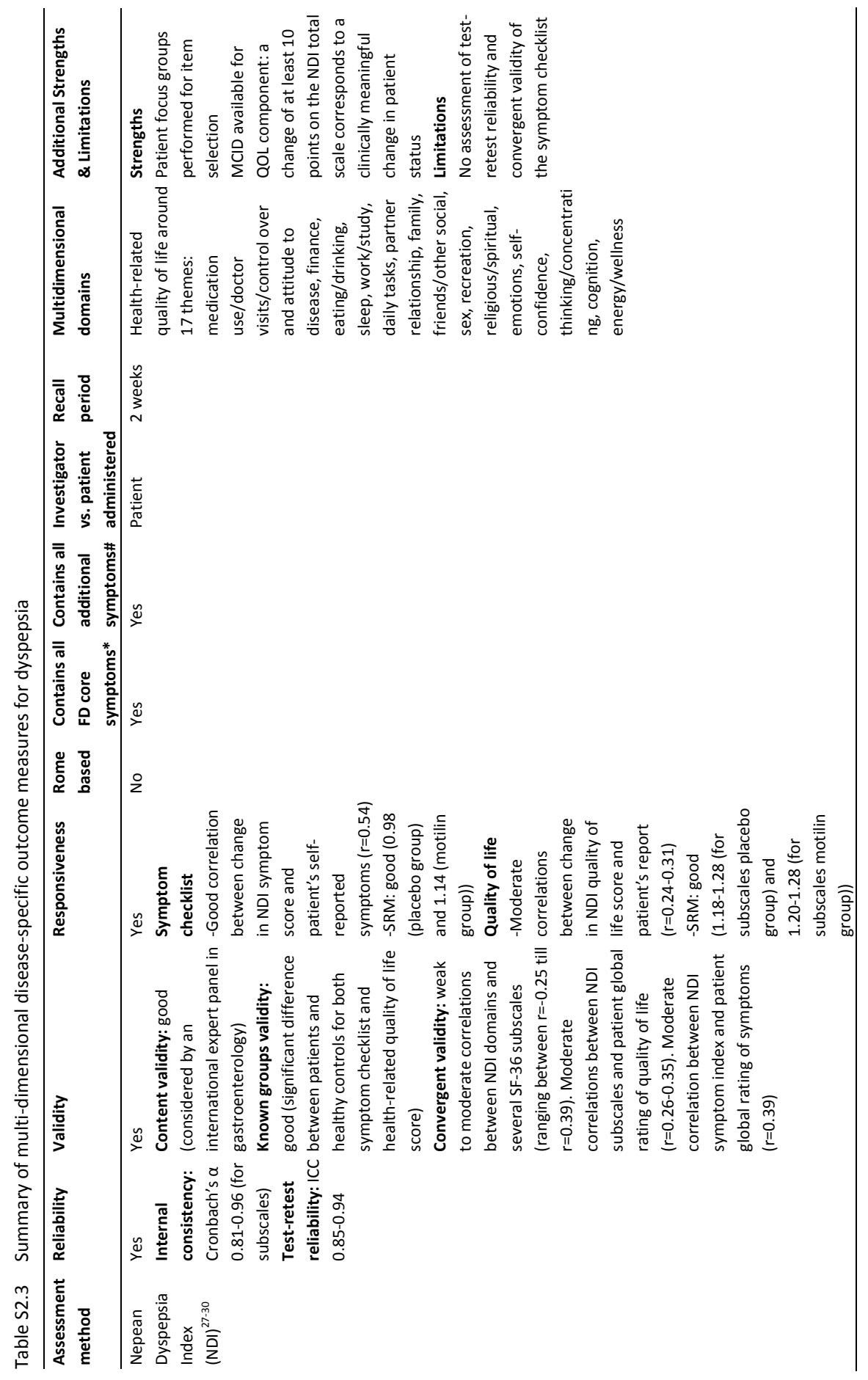




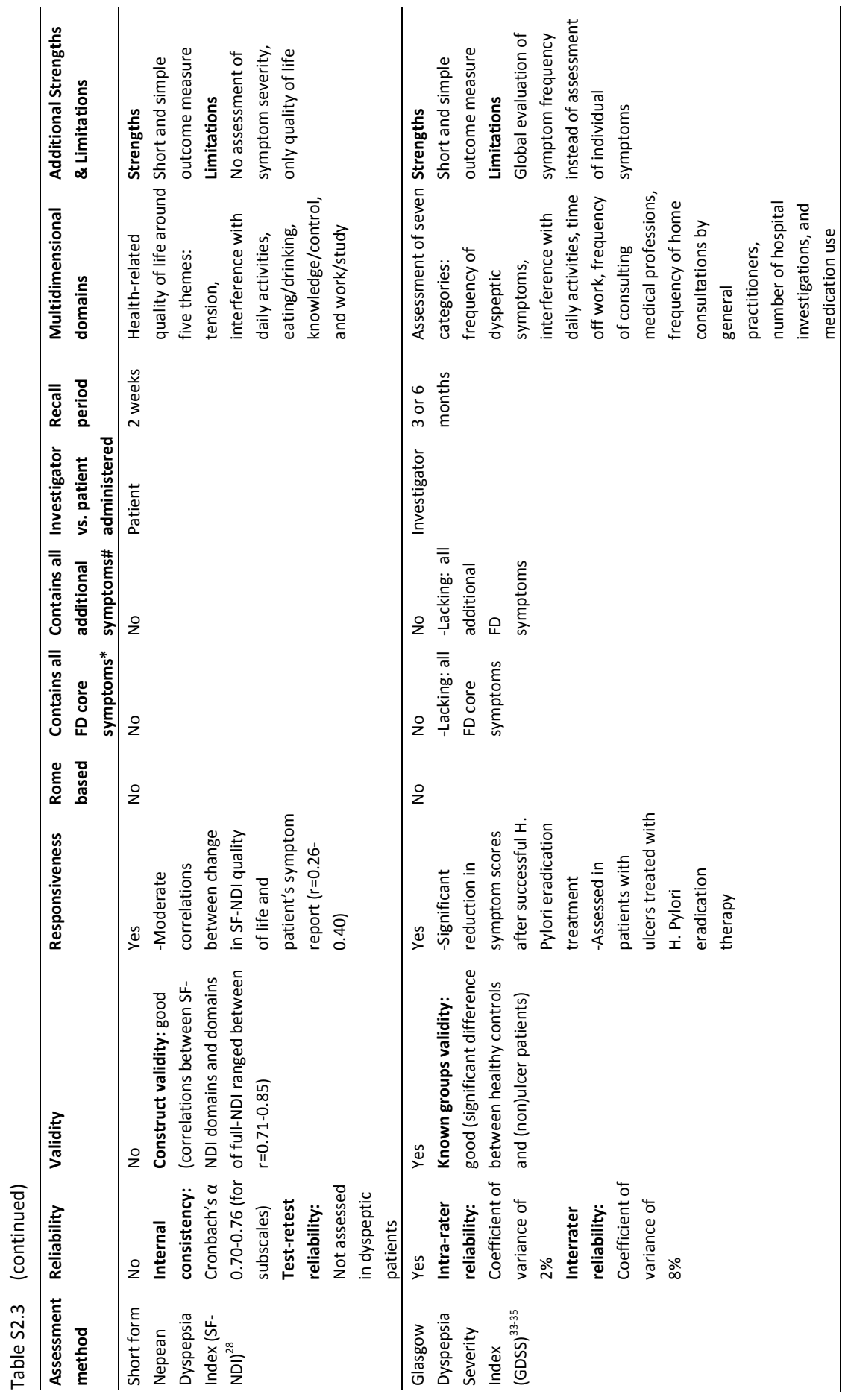




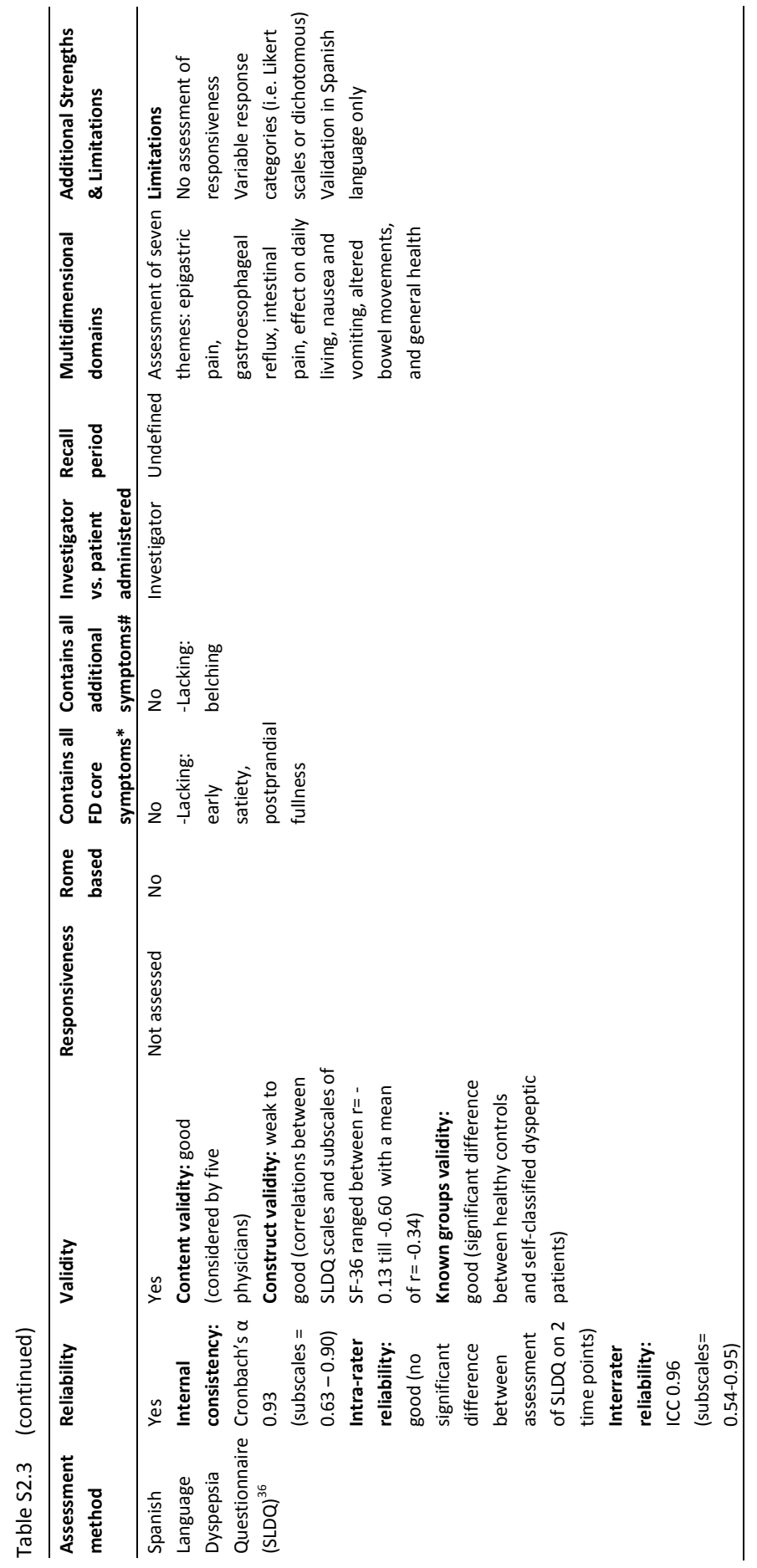




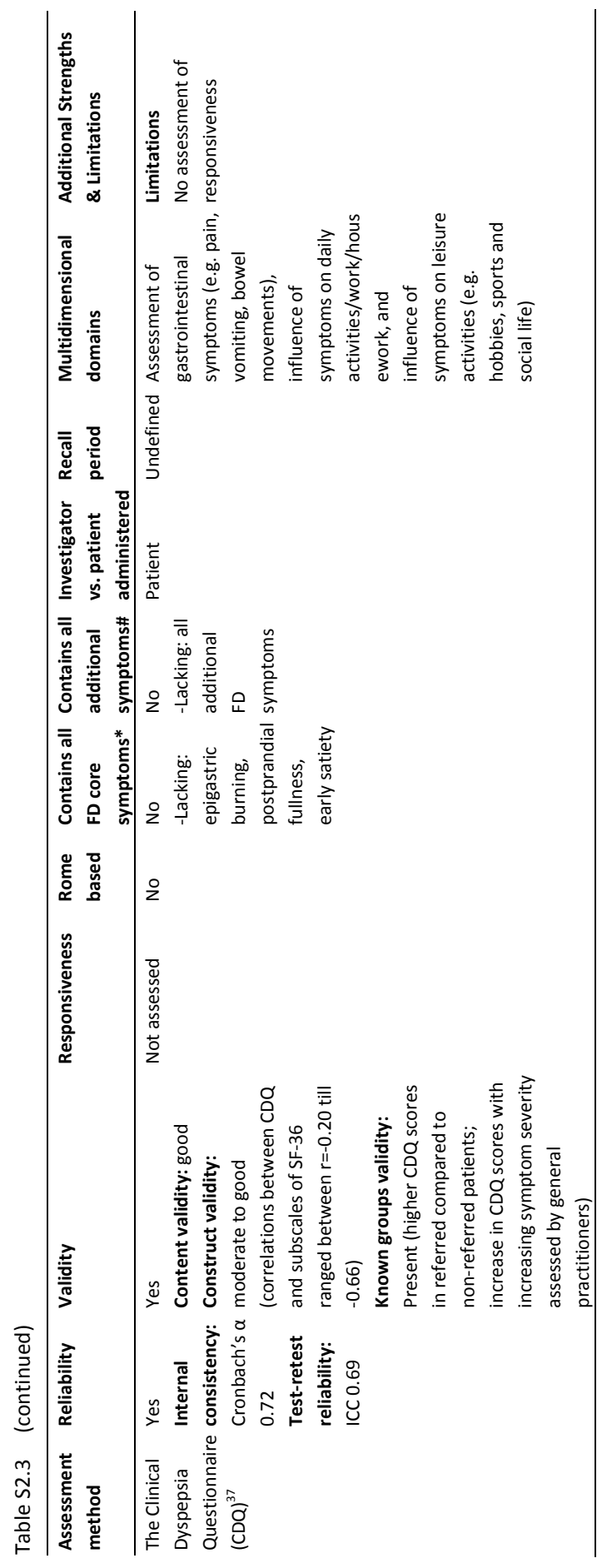




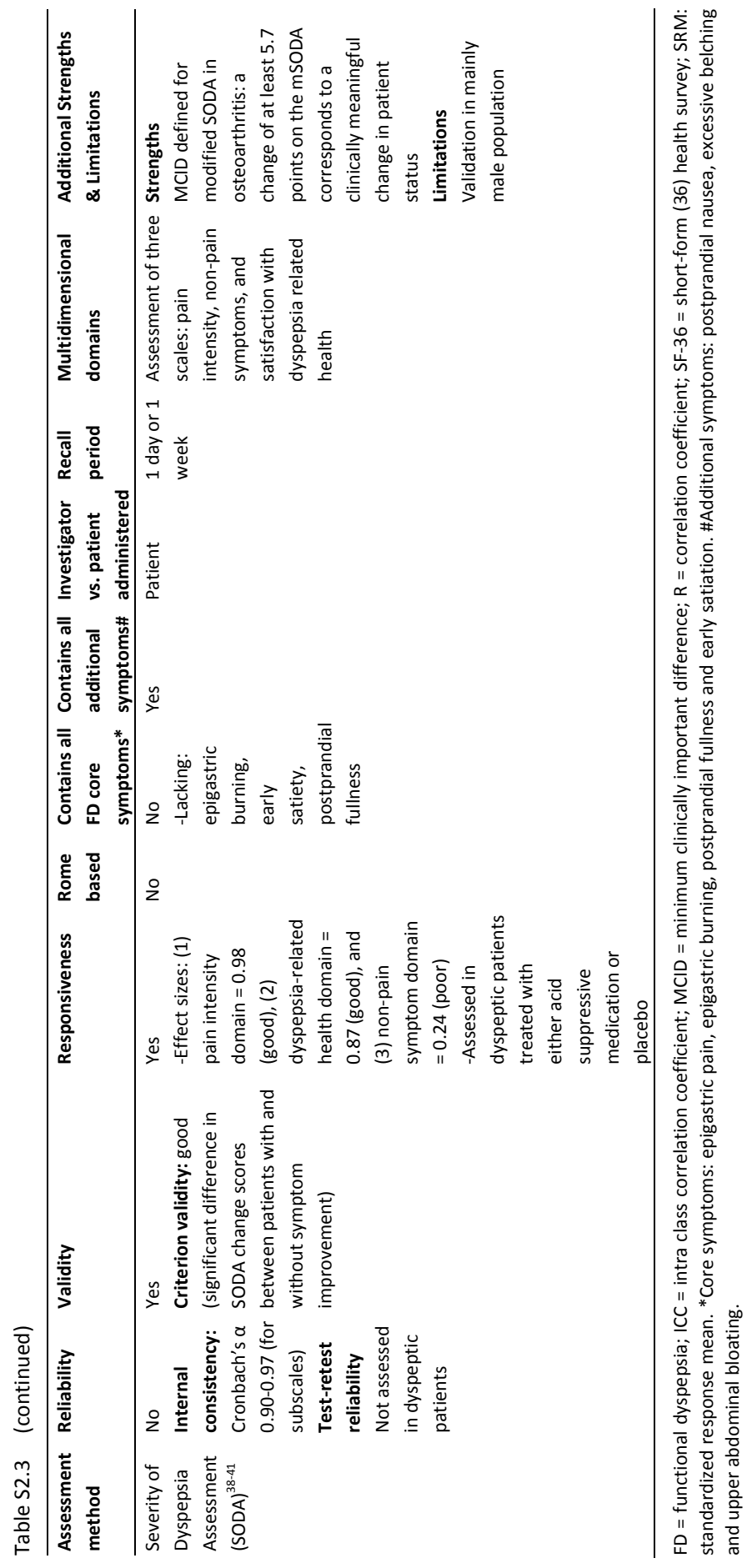




\section{References}

1. Leidy NK, Farup C, Rentz AM, Ganoczy D, Koch KL. Patient-based assessment in dyspepsia: development and validation of Dyspepsia Symptom Severity Index (DSSI). Dig Dis Sci. 2000;45(6): 1172-9.

2. Veldhuyzen van Zanten SCN, Armstrong D. Validation of a 7-point Global Overall Symptom scale to measure the severity of dyspepsia symptoms in clinical trials. Aliment Pharmacol Ther. 2006;23(4): 521-9.

3. Taylor F, Reasner DS, Carson RT, Deal LS, Foley C, lovin R, et al. Development of a symptom-based patient-reported outcome instrument for functional dyspepsia: a preliminary conceptual model and an evaluation of the Adequacy of Existing Instruments. Patient. 2016;9(5):409-18.

4. Moayyedi P, Duffett S, Braunholtz D, Mason S, Richards ID, Dowell AC, et al. The Leeds Dyspepsia Questionnaire: a valid tool for measuring the presence and severity of dyspepsia. Aliment Pharmacol Ther. 1998;12(12):1257-62.

5. Leow HR, Ching SM, Sujarita R, Yap CF, Chia YC, Ho SH, et al. Mandarin version of the Leeds Dyspepsia Questionnaire: A valid instrument for assessing symptoms in Asians. J Dig Dis. 2014;15(11):591-6.

6. Mahadeva S, Chan WK, Mohazmi M, Sujarita R, Goh KL. Validation study of the Leeds Dyspepsia Questionnaire in a multi-ethnic Asian population. J Gastroenterol Hepatol. 2011;26(11):1669-76.

7. Fraser A, Delaney BC, Ford AC, Qume M, Moayyedi P. The Short-Form Leeds Dyspepsia Questionnaire validation study. Aliment Pharmacol Ther. 2007;25(4):477-86.

8. Gatta L, Moayyedi P, Tosetti C, Vakil N, Ubaldi E, Barsanti P, et al. A validation study of the Italian Short-Form Leeds Dyspepsia Questionnaire. Intern Emerg Med. 2010;5(6):501-6.

9. Nkurunziza A, Dusabejambo V, Everhart K, Bensen S, Walker T. Validation of the Kinyarwanda-version Short-Form Leeds Dyspepsia Questionnaire and Short-Form Nepean Dyspepsia Index to assess dyspepsia prevalence and quality-of-life impact in Rwanda. BMJ Open. 2016;6(6):e011018.

10. Svedlund J, Sjodin I, Dotevall G. GSRS--a clinical rating scale for gastrointestinal symptoms in patients with irritable bowel syndrome and peptic ulcer disease. Dig Dis Sci. 1988;33(2):129-34.

11. Talley NJ, Fullerton S, Junghard O, Wiklund I. Quality of life in patients with endoscopy-negative heartburn: reliability and sensitivity of disease-specific instruments. Am J Gastroenterol. 2001; 96(7):1998-2004.

12. Kulich KR, Madisch A, Pacini F, Pique JM, Regula J, Van Rensburg CJ, et al. Reliability and validity of the Gastrointestinal Symptom Rating Scale (GSRS) and Quality of Life in Reflux and Dyspepsia (QOLRAD) questionnaire in dyspepsia: a six-country study. Health Qual Life Outcomes. 2008;6:12.

13. Dimenas E, Glise H, Hallerback B, Hernqvist H, Svedlund J, Wiklund I. Quality of life in patients with upper gastrointestinal symptoms. An improved evaluation of treatment regimens? Scand J Gastroenterol. 1993;28(8):681-7.

14. Hu WH, Lam KF, Wong YH, Lam CL, WM HU, Lai KC, et al. The Hong Kong index of dyspepsia: a validated symptom severity questionnaire for patients with dyspepsia. J Gastroenterol Hepatol. 2002; 17(5):545-51.

15. De Luca L, Zagari RM, Pozzato P, Fiorini T, Ricciardiello L, Martuzzi C, et al. Measuring dyspepsia: a new severity index validated in Bologna. Dig Liver Dis. 2004;36(12):806-10.

16. Sander GB, Mazzoleni LE, Francesconi CF, Wortmann AC, Ott EA, Theil A, et al. Development and validation of a cross-cultural questionnaire to evaluate nonulcer dyspepsia: the Porto Alegre Dyspeptic Symptoms Questionnaire (PADYQ). Dig Dis Sci. 2004;49(11-12):1822-9.

17. Adam B, Liebregts T, Saadat-Gilani K, Vinson B, Holtmann G. Validation of the gastrointestinal symptom score for the assessment of symptoms in patients with functional dyspepsia. Aliment Pharmacol Ther. 2005;22(4):357-63.

18. Crowell MD, Umar SB, Lacy BE, Jones MP, DiBaise JK, Talley NJ. Multi-Dimensional Gastrointestinal Symptom Severity Index: Validation of a Brief GI Symptom Assessment Tool. Dig Dis Sci. 2015; 60(8):2270-9. 
19. Shaw M, Talley NJ, Adlis S, Beebe T, Tomshine P, Healey M. Development of a digestive health status instrument: tests of scaling assumptions, structure and reliability in a primary care population. Aliment Pharmacol Ther. 1998;12(11):1067-78.

20. Shaw MJ, Beebe TJ, Adlis SA, Talley NJ. Reliability and validity of the digestive health status instrument in samples of community, primary care, and gastroenterology patients. Aliment Pharmacol Ther. 2001;15(7):981-7.

21. Carbone F, Holvoet L, Vandenberghe A, Tack J. Functional dyspepsia: outcome of focus groups for the development of a questionnaire for symptom assessment in patients suffering from postprandial distress syndrome (PDS). Neurogastroenterol Motil. 2014;26(9):1266-74.

22. Carbone $F$, Vandenberghe A, Holvoet L, Vanuytsel T, Van Oudenhove L, Jones $M$, et al. Validation of the Leuven Postprandial Distress Scale, a questionnaire for symptom assessment in the functional dyspepsia/postprandial distress syndrome. Aliment Pharmacol Ther. 2016;44(9):989-1001.

23. (FDA) FaDA. Guidance for Industry; Patient-Reported Outcome Measures: use in Medical Product Development to Support Labeling Claims 2009.

24. Taylor F, Higgins S, Carson RT, Eremenco S, Foley C, Lacy BE, et al. Development of a symptomfocused patient-reported outcome measure for functional dyspepsia: The Functional Dyspepsia Symptom Diary (FDSD). Am J Gastroenterol. 2018;113(1):39-48.

25. Rentz AM, Kahrilas P, Stanghellini V, Tack J, Talley NJ, de la Loge C, et al. Development and psychometric evaluation of the patient assessment of upper gastrointestinal symptom severity index (PAGI-SYM) in patients with upper gastrointestinal disorders. Qual Life Res. 2004;13(10):1737-49.

26. Revicki DA, Rentz AM, Tack J, Stanghellini V, Talley NJ, Kahrilas P, et al. Responsiveness and interpretation of a symptom severity index specific to upper gastrointestinal disorders. Clin Gastroenterol Hepatol. 2004;2(9):769-77.

27. Talley NJ, Haque M, Wyeth JW, Stace NH, Tytgat GN, Stanghellini V, et al. Development of a new dyspepsia impact scale: the Nepean Dyspepsia Index. Aliment Pharmacol Ther. 1999;13(2):225-35.

28. Talley NJ, Verlinden M, Jones M. Quality of life in functional dyspepsia: responsiveness of the Nepean Dyspepsia Index and development of a new 10-item short form. Aliment Pharmacol Ther. 2001; 15(2):207-16.

29. Talley NJ, Verlinden M, Jones M. Validity of a new quality of life scale for functional dyspepsia: a United States multicenter trial of the Nepean Dyspepsia Index. Am J Gastroenterol. 1999;94(9): 2390-7.

30. Jones M, Talley NJ. Minimum clinically important difference for the Nepean Dyspepsia Index, a validated quality of life scale for functional dyspepsia. Am J Gastroenterol. 2009;104(6):1483-8.

31. Tian XP, Li Y, Liang FR, Sun GJ, Yan J, Chang XR, et al. Translation and validation of the Nepean Dyspepsia Index for functional dyspepsia in China. World J Gastroenterol. 2009;15(25):3173-7.

32. Mahadeva S, Wee HL, Goh KL, Thumboo J. Quality of life in South East Asian patients who consult for dyspepsia: validation of the short form Nepean Dyspepsia Index. Health Qual Life Outcomes. 2009;7:45.

33. el-Omar EM, Banerjee S, Wirz A, McColl KE. The Glasgow Dyspepsia Severity Score--a tool for the global measurement of dyspepsia. Eur J Gastroenterol Hepatol. 1996;8(10):967-71.

34. Calvet X, Bustamante E, Montserrat A, Roque M, Campo R, Gene E, et al. Validation of phone interview for follow-up in clinical trials on dyspepsia: evaluation of the Glasgow Dyspepsia Severity Score and a Likert-scale symptoms test. Eur J Gastroenterol Hepatol. 2000;12(8):949-53.

35. Talley NJ, Agreus I, Veldhuyzen van Zanten S. Is the Glasgow Dyspepsia Severity Score a tool for the global measurement of dyspepsia? Eur J Gastroenterol Hepatol. 1997;9(4):413-4.

36. Goldman J, Conrad DF, Ley C, Halperin D, de la Luz Sanchez M, Villacorta R, et al. Validation of Spanish language dyspepsia questionnaire. Dig Dis Sci. 2002;47(3):624-40.

37. Garratt AM, Ruta DA, Russell I, Macleod K, Brunt P, McKinlay A, et al. Developing a condition-specific measure of health for patients with dyspepsia and ulcer-related symptoms. J Clin Epidemiol. 1996;49(5):565-71.

38. Kuykendall DH, Rabeneck L, Campbell CJ, Wray NP. Dyspepsia: how should we measure it? J Clin Epidemiol. 1998;51(2):99-106. 
39. Cook KF, Rabeneck L, Campbell CJ, Wray NP. Evaluation of a multidimensional measure of dyspepsiarelated health for use in a randomized clinical trial. J Clin Epidemiol. 1999;52(5): 381-92.

40. Rabeneck L, Cook KF, Wristers K, Souchek J, Menke T, Wray NP. SODA (severity of dyspepsia assessment): a new effective outcome measure for dyspepsia-related health. J Clin Epidemiol. 2001;54(8):755-65.

41. Rabeneck L, Wristers K, Goldstein JL, Eisen G, Dedhiya SD, Burke TA. Reliability, validity, and responsiveness of severity of dyspepsia assessment (SODA) in a randomized clinical trial of a COX-2specific inhibitor and traditional NSAID therapy. Am J Gastroenterol. 2002;97(1):32-9.

42. Welle J, Fort J, Crawley J, Cryer B, Dickerhoof R, Turner MP, et al. Modified Severity of Dyspepsia Assessment pain scale: a new tool for measuring upper abdominal pain in osteoarthritis patients taking NSAIDs. Patient Relat Outcome Meas. 2011;2:135-43.

43. Kindt S, Dubois D, Van Oudenhove L, Caenepeel P, Arts J, Bisschops R, et al. Relationship between symptom pattern, assessed by the PAGI-SYM questionnaire, and gastric sensorimotor dysfunction in functional dyspepsia. Neurogastroenterol Motil. 2009;21(11):1183-e105. 


\section{Chapter 3}

Development of a real-time patient-reported outcome measure for symptom assessment in patients with functional dyspepsia using the experience sampling method

F.G.M. Smeets, D. Keszthelyi, L. Vork, J. Tack, N.J. Talley, M. Simren, Q. Aziz, A.C. Ford, J.M. Conchillo, J.W. Kruimel, J. Van Os, A.A.M. Masclee Neurogastroenterol Motil. 2019;31(2):e13496 


\section{Abstract}

\section{Background}

Patient-reported outcome measures (PROMs) are used to assess symptoms in patients with functional dyspepsia. Current end-of-day questionnaires have several limitations including sensitivity to recall and ecological bias. The Experience Sampling Method (ESM) is characterized by random and repeated assessments across momentary states in daily life and therefore less sensitive to these limitations. This study describes the development of a novel PROM based on ESM technology.

\section{Methods}

An initial draft of the PROM was developed based on literature. Focus group interviews with FD patients according to Rome IV criteria, and an expert meeting with international opinion leaders in the field of functional gastrointestinal disorders were conducted in order to select items for the PROM. Cognitive interviews were performed to evaluate patients' understanding of the selected items and to create the definitive PROM.

\section{Key results}

A systematic literature search revealed 59 items across four domains (i.e. physical status, mood and psychological factors, context and environment, and nutrition, medication and substance use). After patient focus group interviews and an international expert meeting, the number of items was reduced to 33. Cognitive interviews resulted in some minor linguistic changes in order to improve patients' understanding.

\section{Conclusions \& Inferences}

A novel digital ESM-based PROM for real-time symptom assessment in patients with functional dyspepsia was developed. This novel PROM has the potential to identify individual symptom patterns and specific triggers for dyspeptic symptoms, and optimize treatment strategies. 


\section{Introduction}

Functional dyspepsia (FD) is a common functional gastrointestinal disorder with an estimated prevalence of $8-12 \%$ in the general population. ${ }^{1-3}$ Symptom presentation of dyspeptic patients is heterogeneous although four core symptoms have been defined according to the Rome IV criteria: postprandial fullness, early satiation, epigastric burning and epigastric pain. ${ }^{4,5}$ These symptoms lead to impaired quality of life, reduced work productivity and increased health care costs, which underlines the need for (development of) effective treatment options. ${ }^{5-7}$

Functional dyspepsia is a symptom-based diagnosis and patient-reported outcome measures (PROMs) are used to assess treatment efficacy. ${ }^{8}$ In a recently published systematic review, twenty available retrospective outcome measures were described for the evaluation of dyspeptic symptoms. ${ }^{9}$ However, these outcome measures do not fulfill all criteria for adequate psychometric validation, as defined by regulatory authorities.

Moreover, several limitations of retrospective end-of-day questionnaires are apparent. First, retrospective outcome measures are prone to recall bias as retrieval of information is based on autobiographical memory. ${ }^{10}$ Second, dyspeptic symptoms vary over time due to the influence of certain circumstances and triggers (e.g. food intake and psychosocial factors). ${ }^{11,12}$ Lack of ecological validity may occur when questionnaires are completed in another environment or situation, compared with situations in which symptoms were triggered. ${ }^{13}$ Third, non-compliance is a major limitation of retrospective paper questionnaires, and this could potentially be eliminated by use of an electronic sampling method. ${ }^{14}$ These limitations of available retrospective questionnaires together with the lack of a single universally accepted PROM, underline the need for development of a novel PROM.

The Experience Sampling Method (ESM) is an attractive electronic method for real-time assessment of symptoms, which may overcome some of the limitations of retrospective questionnaires. ${ }^{13}$ Random, repeated assessments are used for several consecutive days to capture symptom variability over time, and this takes into account contextual, social and psychological factors, which might influence dyspeptic symptoms. ${ }^{13}$ Although ESM has been applied in several disorders (e.g. mental disorders), use in patients with functional gastrointestinal diseases is currently limited to two studies in patients with irritable bowel syndrome (IBS). ${ }^{15-17}$ Both studies reported higher symptom scores in end-of-day diaries when compared to day-average scores of momentary assessments with ESM. ${ }^{16,17}$

However, the ESM has not been previously applied in patients with functional dyspepsia. In this report we describe the development of a novel PROM based on ESM technology for real-time assessment of symptoms in patients with functional dyspepsia. 


\section{Methods}

Development of a novel ESM-based PROM for symptom assessment in patients with functional dyspepsia was undertaken in several stages according to the FDA guidelines for the development of PROMs. ${ }^{8}$

\section{Phase I: Item selection}

A conceptual framework of theoretical constructs was developed for assessment of symptoms in patients with functional dyspepsia. For development of this framework, an extensive systematic literature search was performed to obtain available retrospective questionnaires for selection of FD-specific items. ${ }^{9}$ Moreover, ESMspecific constructs (i.e. psychological status, social, and contextual factors) were derived from previously used ESM questionnaires at the departments of Gastroenterology and Hepatology, and Psychiatry and Psychology of Maastricht University Medical Center (MUMC+). ${ }^{17,18}$

\section{Phase II: Focus group interviews}

\section{Selection of participants for focus groups}

Consecutive ambulatory patients between the age of 18 and 75 years with a diagnosis of functional dyspepsia were recruited at the outpatient department of Gastroenterology and Hepatology at Maastricht University Medical Center (MUMC+). Patients were diagnosed with functional dyspepsia according to Rome IV criteria and an upper gastrointestinal endoscopy was performed up to 12 months prior to the focus group interview in order to exclude organic abnormalities. ${ }^{5}$ Patients with FD and a comorbid diagnosis of irritable bowel syndrome (IBS) were not excluded due to the considerable overlap of both disorders, and to acquire a representative sample of the general population. Participants spoke Dutch as their mother tongue as focus groups were conducted in Dutch. All participants gave written informed consent and the study protocol was approved by the medical ethical committee of Maastricht University Medical Center (METC 17-4-056).

\section{Conducting and moderating focus group interviews}

Patient focus group sessions were organized in order to identify a relevant set of items for symptom assessment in functional dyspepsia and discuss practical issues of the ESM-procedure.

Eligible participants were invited to participate in a focus group meeting which took approximately 90 minutes. Focus group interviews were planned and continued 
until saturation of input was achieved. ${ }^{19}$ Preferably, five to ten participants were included per focus group, in order to obtain the full spectrum of perspectives. ${ }^{19,20}$

After an introduction about the aim of the study, one moderator (F.S.) and one assistant-moderator (L.V.) guided the focus groups with use of a power point presentation to ensure adequate consistency in interview content. In order to minimize the probability of bias induced by the moderator, the first part of the focus group consisted of an open discussion in which participants were questioned about which items they considered to be essential in an outcome measure for dyspeptic patients. This section of the focus group aimed to (1) acquire information about experiences of gastrointestinal symptoms, (2) obtain information about the influence of symptoms on daily life, and (3) identify which factors or triggers influence dyspeptic symptoms.

During the second part of the focus group, 59 items selected from previously used outcome measures and ESM questionnaires were presented on the power point presentation, and participants were asked to criticize these items. They were asked to consider which items were relevant in a real-time symptom assessment method for functional dyspepsia, and which items could be excluded. Moreover, patients were asked to identify incomprehensible items and provide alternative terms or descriptions. Care was taken to obtain feedback from all participants on each item, and the number of participants that considered each item relevant was assessed. At the end of each domain open-ended questions were asked to evaluate whether additional symptoms required mentioning.

Fatigue and sleeping problems are frequently mentioned by patients with functional gastrointestinal complaints. Assessment of sleep and fatigue is however not suitable for repeated assessment over the day. In line with the recently developed ESM-based IBS PROM, we intended to include a 'morning' questionnaire for assessment of sleep once a day.

Besides evaluation of items to be included in an outcome measure for assessment of dyspeptic symptoms, several practical components of the ESM procedure were discussed. The proposed 11-point (0-10) Numeric Rating Scale (NRS), which is currently recommended for use as endpoint in clinical trials of patients with IBS, was discussed. ${ }^{21}$ Second, the number of assessments during the day and the time spent for completing each assessment (i.e. 3-5 minutes) were discussed to assess the feasibility and burden of the ESM-based outcome measure. Random and repeated symptom assessment is the key feature of ESM. Ten times a day a beep signal is produced by the application on the smartphone between 07.30 and 22.30 hour. Auditory cues are submitted in 90-minute blocks, and the minimum interval between two beeps is 15 minutes. Beeps occur completely random without relation to meal intake in order to prevent anticipation of symptom assessment. 


\section{Phase III: Expert meeting}

After conducting the focus groups, a teleconference meeting was organized with international experts in the fields to discuss the relevance of the individual items that were selected from the systematic literature search and patient focus groups. Experts criticized the items regarding their relevance and suitability for a real-time symptom assessment method. Based on this discussion, a definitive draft with questions for the ESM-based outcome measure was developed.

\section{Phase IV: Cognitive interviews}

Interviews focused on cognitive understanding were performed to evaluate patients' grasp of the items included in the definitive draft. Again, native Dutch-speaking patients with functional dyspepsia according to the Rome IV criteria were included, but who had not previously taken part in the focus group interviews. The definitive ESM-draft was presented to the participants on paper. Participants were asked to read the items, speak them out loud, and consider whether the meaning of the items was clear (i.e. verbal probing). ${ }^{22}$ Moreover, participants were asked for recommendations to improve the outcome measure. Cognitive interviews were performed on a one-to-one basis, and were continued until additional cognitive interviews did not lead to substantial suggestions or recommendations from the participants.

\section{Data analysis}

\section{Analysis of focus group data}

Focus groups were recorded by a voice-recorder and notes were taken by both the moderator and assistant-moderator. Immediately after the focus group sessions, a debriefing was performed between the moderator and assistant-moderator to review and summarize the acquired information and observations. ${ }^{19,20}$ In addition, voice recordings were transcribed verbatim and summarized in a database together with the moderators' notes. This database was used to identify saturation of input, which is achieved when the core symptoms discussed in the sessions are stable, and subsequent focus groups only produce repetitive information. ${ }^{19,23}$ Finally, the database was used to decide which items should be included in the definitive draft instrument.

\section{Analysis of cognitive grasp interviews}

For the analysis of interviews focusing on cognitive grasp, a database was constructed to transcribe and summarize participants' statements, in order to facilitate 
comparison of interpretations for all individual items. This information was used to decide whether any modification of items was necessary. ${ }^{22}$

\section{Results}

\section{Phase I: Item selection}

Based on a systematic literature search and collection of previously used ESM questionnaires, 59 items were included in the initial ESM instrument. These items were divided into four domains: (1) physical status, (2) mood, (3) context and environment, and (4) nutrition, medication and substance use (Figure 3.1). The "physical status" domain could be divided into three categories: (1) upper gastrointestinal symptoms, including symptoms of dyspepsia, gastroesophageal reflux disease, and other disorders (e.g. nausea, belching, vomiting), (2) lower gastrointestinal symptoms, and (3) general physical complaints.

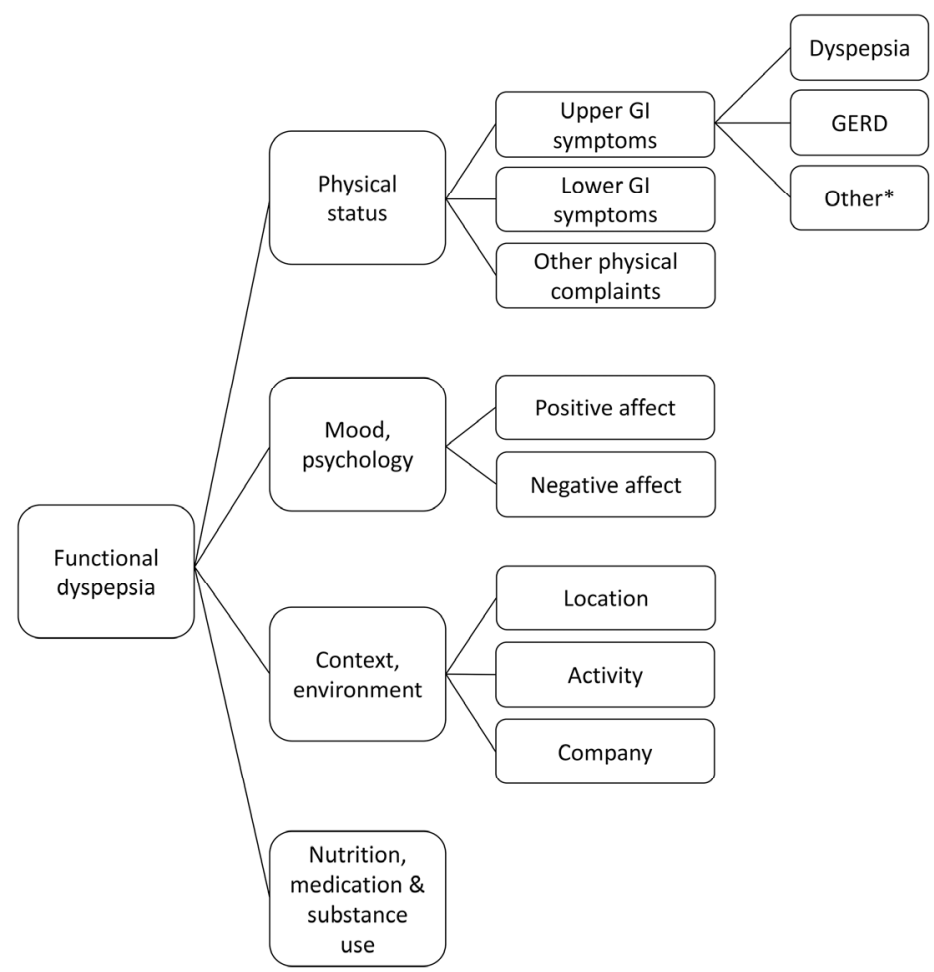

Figure 3.1 Conceptual framework for development of a novel patient-reported outcome measure using the Experience Sampling Technology.

* Nausea, vomiting, belching. GI = gastrointestinal, GERD = gastroesophageal reflux disease. 


\section{Phase II: Focus group interviews}

\section{Study population}

Forty-four patients with functional dyspepsia according to Rome IV criteria were invited for the focus group meetings. Eighteen patients confirmed their participation, and fourteen patients were present during three different focus group meetings (group 1: $n=6$, group 2: $n=3$, group $3: n=5)$. Family circumstances $(n=2)$ and lack of time $(n=2)$ were reasons for cancelation. Patient characteristics are described in Table 3.1.

Table 3.1 Baseline characteristics of patients which participated in focus group interviews and cognitive interviews.

\begin{tabular}{|c|c|c|}
\hline & $\begin{array}{l}\text { Participants focus group } \\
\text { interviews }(n=14)\end{array}$ & $\begin{array}{l}\text { Participants cognitive } \\
\text { interviews }(n=6)\end{array}$ \\
\hline Age in years, median (range) & $64(39-73)$ & $47(27-58)$ \\
\hline Female, $\mathrm{n}(\%)$ & $10(71 \%)$ & $5(83 \%)$ \\
\hline \multicolumn{3}{|c|}{ FD-subtype based on Rome IV criteria, $\mathrm{n}(\%)$} \\
\hline FD-PDS* & $1(7 \%)$ & $3(50 \%)$ \\
\hline FD-EPS & $6(43 \%)$ & $1(17 \%)$ \\
\hline Mixed subtype & $7(50 \%)$ & $2(33 \%)$ \\
\hline Comorbid diagnosis of IBS, $n$ (\%) & $3(21 \%)$ & $1(17 \%)$ \\
\hline
\end{tabular}

* FD-PDS: functional dyspepsia of the postprandial distress subtype; $¥$ FD-EPS: functional dyspepsia of the epigastric pain subtype.

\section{Focus group interviews}

\section{Physical status}

- $\quad$ Upper abdominal symptoms

Postprandial fullness, early satiation, epigastric pain, and epigastric burning were considered important symptoms, and were described by the majority of patients (86\%, 64\%, 64\% and $86 \%$ respectively). Participants considered two aspects essential for real-time symptom assessment of pain: (1) pain severity and (2) location of pain. Assessment of pain severity using an 11-point NRS was considered adequate. To localize pain, participants suggested to add a schematic picture of the abdomen in which study subjects could select separate abdominal regions. In addition, several participants suggested description of the character of the pain (e.g. cramp-like, dull) using multiple-choice options.

In addition to the four core FD symptoms, participants agreed on the importance of scoring additional frequently occurring upper gastrointestinal symptoms including bloating (86\%), nausea (100\%), and belching (93\%). The open discussion revealed that bloating was sometimes considered equivalent to postprandial 
fullness. After clarification of the meaning of bloating (i.e. an unpleasant sensation of gaseous distension), patients considered this an essential item for real-time symptom assessment separate from postprandial fullness. Not only bloating, but also nausea required some further explanation as several participants correlated nausea with general malaise, instead of a desire to vomit. Heartburn was described by 12 patients (86\%) and was considered distinct from epigastric pain.

- Lower abdominal symptoms

In addition to upper gastrointestinal symptoms, four participants reported lower abdominal pain and three patients had a comorbid diagnosis of IBS according to the Rome IV criteria. As a consequence, assessment of lower abdominal pain, defecation frequency, and consistency were considered important by four and three patients respectively.

- General complaints

Several non-abdominal complaints were considered relevant by the majority of participants including palpitations, sweating, shortness of breath, and dizziness. Moreover, fatigue was reported by all participants and was considered an essential question. However, due to the often long lasting nature of fatigue, this item was considered not eligible for repeated assessment. However, assessment of sleep with a 'morning' questionnaire once a day was considered important by participants. Complaints reported by individual subjects were headache, weight loss, and fainting.

\section{Mood and psychological factors}

Assessment of psychological factors was considered important by all subjects, as they often experienced an association between psychological factors and gastrointestinal symptoms. 'I feel anxious' (79\%), 'I feel irritated' (79\%), and 'I feel stressed' (100\%) were considered important negative emotions; whereas 'I feel relaxed' (100\%) was considered a relevant positive affect. Participants discussed the item 'I feel excited' as several patients found this item too positive and preferred 'I feel good right now'.

\section{Context and environment}

Contextual and environmental factors were considered relevant as these might influence the presence and/or severity of gastrointestinal symptoms. Questions with regard to location ('Where am I?'), activity ('What am I doing?'), and company ('Who is with me?'), with multiple choice answers, were considered important by all participants. In addition, the majority of patients (86\%) found it important to ask whether participants were restricted in their daily activities by current complaints. 
Nutrition, medication and substance use

Participants stressed that dietary factors and medication use should be taken into account when assessing dyspeptic symptoms. Two components of nutrition were considered essential, namely (1) type of food products, and (2) amount of food intake. All subjects agreed on the question 'Since the last beep I used ....' with categorical answers (i.e. breakfast, lunch, dinner, or a snack). As several types of food and substance use can trigger symptoms, the question 'What did you use since the last beep?' with categorical answer options (i.e. coffee, tea, nicotine, drugs, medication, carbonated beverages) was considered appropriate to all participants. In patients who took medication, a categorical answer option was preferable (i.e. painkillers for abdominal pain, painkillers for another cause, acid suppressive medication, antiemetics, or something else). Although early satiety is a core symptom of FD, assessment of the amount of food intake is difficult. We suggested 'I was able to finish a normal sized meal', which was appropriate for participants.

\section{Practical issues}

Several practical issues relating to the ESM procedure were discussed. An 11-point NRS was considered adequate and appropriate to grade the presence and severity of individual symptoms. Divergent opinions were present with regard to the proposed number of 10 measurements per day. A subgroup of participants indicated that 10 assessments per day would be quite burdensome and suggested that five to six measurements per day would be more acceptable. However, other participants considered it an investment in order to get additional information about their disorder and potentially optimize treatment.

Moreover, participants indicated that a 3 to 5-minute period was acceptable for completing each questionnaire. In order to reduce the time needed to complete the questionnaires, several participants suggested the use of an algorithm, in order to skip questions if possible. For instance, if participants answer ' 0 times' on the question 'How many times did you defecate since the previous assessment?' then the question with regard to stool consistency could be omitted.

\section{Phase III: International expert meeting}

All items resulting from the previous phases of the project were discussed by international experts in the field to assess the relevance for inclusion of individual items in an ESM-based FD-PROM.

With regard to upper gastrointestinal symptoms, several items were excluded as they were considered not relevant for inclusion in an FD-specific questionnaire (e.g. dysphagia) or were not eligible for repeated assessments (e.g. loss of appetite). In addition, some nuances were added for several items. For instance, patients in focus 
group interviews could not agree whether 'I am having a full feeling' and 'I am having a heavy feeling' were considered as identical or separate symptoms. Therefore, experts suggested to include both items and evaluate them in a future validation study. Moreover, experts decided to merge 'nausea' and 'the feeling that I have to vomit', and recommended that a schematic pictogram of the abdomen should be given to patients before the start of the ESM-questionnaire, in order to define the 'upper' and 'lower' abdomen.

Inclusion of items related to the lower gastrointestinal tract was extensively debated, as opinions of the international experts were heterogeneous. Although the goal was to develop an FD-specific PROM, considerable overlap is present between FD and IBS, which was also demonstrated in the patient focus groups, as $21 \%$ had a comorbid diagnosis of IBS. As it was supposed that it would be beneficial to evaluate the (potential) relation between upper and lower gastrointestinal symptoms with this ESM-principle, experts agreed with inclusion of the most important lower gastrointestinal items (i.e. lower abdominal pain, number and consistency of bowel movements, and incomplete evacuation).

The international experts agreed on inclusion of general physical complaints, as previous studies found associations between dyspeptic symptoms and somatic complaints.

Selection and inclusion of psychological items was extensively discussed as these ESM-psychological items have not been validated before in patients with gastrointestinal disorders. Item selection was therefore performed based on a recent publication evaluating psychometric validation properties of the ESM in patients with mental disorders, the previously developed ESM-IBS questionnaire, together with patients' opinion in focus group interviews. ${ }^{18,24}$

Contextual items were discussed as well, but no changes were made. Moreover, experts agreed that nutritional items were of special interest with regard to dyspeptic symptoms. Although patients in the focus groups mentioned specific kinds of food as triggers for dyspeptic symptoms (e.g. onions, spicy food), evaluation of associations between dyspeptic symptoms and specific kinds of food was considered beyond the scope of this project.

In conclusion, focus group interviews and an international expert meeting resulted in a reduction of the number of included items from 59 to 33, categorized in the before-mentioned four domains.

\section{Phase IV: Cognitive grasp interviews}

Demographic characteristics of the participants are shown in Table 3.1. One participant had a comorbid diagnosis of IBS according to Rome IV criteria. Only a few minor modifications were suggested to improve patients' understanding with the 
items. For instance, the wording of items regarding dyspnea and chest pain were modified.

The final ESM-based instrument for repeated symptom assessment is shown in Table 3.2. In addition, the morning questionnaire for assessment once a day is shown in Table 3.3. Content validity was assessed according to the following items: (1) development of a conceptual framework, (2) extensive item generation by a systematic literature search and organisation of patient focus group interviews in the target population of FD patients according to Rome IV criteria with use of open-ended questions, continuation until saturation of input was achieved and verbatim transcription, (3) discussion of practical issues of the novel ESM-based PROM during focus group interviews (e.g. digital mode of data collection, number of assessments per day, duration of data collection per day, recall period and response scale), (4) teleconference with international experts in the field of functional dyspepsia and ESM, and (5) cognitive interviews in order to assess the participants' understanding of the PROM and evaluate the comprehensiveness of content.

Table 3.2 Definite set of items for the ESM-PROM.

\begin{tabular}{|c|c|c|}
\hline & Physical status - Upper abdomen & Answer scale \\
\hline 1 & I am having a full feeling in my upper abdomen & 0 (none) - 10 (very much) \\
\hline 2 & I am having a heavy feeling in my upper abdomen & 0 (none) - 10 (very much) \\
\hline 3 & My abdomen feels bloated & 0 (none) - 10 (very much) \\
\hline 4 & I am having pain in my upper abdomen & 0 (none) - 10 (very much) \\
\hline 5 & I am having a burning feeling in my upper abdomen & 0 (none) - 10 (very much) \\
\hline 6 & I am having pain in my lower abdomen & 0 (none) - 10 (very much) \\
\hline 7 & I feel nauseous & 0 (none) - 10 (very much) \\
\hline $7 a$ & Since the last beep, I have actually vomited .. time(s) & $0,1,2,3,4,5$, more than 5 times \\
\hline 8 & I am suffering from bothersome burping & 0 (none) - 10 (very much) \\
\hline 9 & I am having a burning feeling behind the breastbone & 0 (none) - 10 (very much) \\
\hline \multirow[t]{2}{*}{10} & I am bringing up stomach contents into the mouth and/or nose & 0 (none) - 10 (very much) \\
\hline & Nutrition, medication and substance use & \\
\hline 11 & I have eaten ... since the last beep & $\begin{array}{l}\text { Breakfast, lunch, dinner, a snack, } \\
\text { none of these }\end{array}$ \\
\hline $11 \mathrm{a}$ & I ate ... ago & $\begin{array}{l}\text { Less than } 15 \text { min., } 15-30 \text { min., } 30 \\
\text { min.-1 hour, more than } 1 \text { hour }\end{array}$ \\
\hline $11 b$ & I am able to finish a normal sized meal & 0 (none) - 10 (very much) \\
\hline 12 & Since the last beep I have used ... & $\begin{array}{l}\text { Caffeine (coffee) / theine (tea), } \\
\text { nicotine (smoking), alcohol, drugs, } \\
\text { medication, carbonated beverages, } \\
\text { milk products, fruit juices, none of } \\
\text { these }\end{array}$ \\
\hline $12 a$ & This was medication for: & $\begin{array}{l}\text { abdominal pain, other pain relief, } \\
\text { stomach acidity, nausea, diarrhea, } \\
\text { constipation, something else }\end{array}$ \\
\hline
\end{tabular}


Table 3.2 (continued)

\begin{tabular}{|c|c|c|}
\hline & Physical status - General complaints & \\
\hline 13 & I am having palpitations & 0 (none) - 10 (very much) \\
\hline 14 & I am sweating & 0 (none) - 10 (very much) \\
\hline 15 & I am short of breath & 0 (none) - 10 (very much) \\
\hline 16 & I feel dizzy & 0 (none) - 10 (very much) \\
\hline 17 & I have pain on my chest & 0 (none) - 10 (very much) \\
\hline 18 & I feel tired & 0 (none) - 10 (very much) \\
\hline 19 & $\begin{array}{l}\text { Since the last beep, I have had the feeling that I had to open } \\
\text { my bowels ... time(s) }\end{array}$ & $0,1,2,3,4,5$, more than 5 times \\
\hline 20 & $\begin{array}{l}\text { Since the last beep, I have actually opened my bowels ... } \\
\text { time(s) }\end{array}$ & $0,1,2,3,4,5$, more than 5 times \\
\hline $20 a$ & It looked like this: & Bristol Stool Form Scale \\
\hline $20 \mathrm{~b}$ & I had to strain & 0 (none) - 10 (very much) \\
\hline \multirow[t]{2}{*}{ 20c } & It feels like my bowels are not completely empty & 0 (none) - 10 (very much) \\
\hline & Psychological aspects & \\
\hline 21 & I am feeling good & 0 (none) - 10 (very much) \\
\hline 22 & I am feeling relaxed & 0 (none) - 10 (very much) \\
\hline 23 & I am feeling low & 0 (none) - 10 (very much) \\
\hline 24 & I am feeling anxious & 0 (none) - 10 (very much) \\
\hline 25 & I am feeling irritated & 0 (none) - 10 (very much) \\
\hline 26 & I am feeling stressed & 0 (none) - 10 (very much) \\
\hline \multirow[t]{2}{*}{27} & I am worried & 0 (none) - 10 (very much) \\
\hline & Context and environment & \\
\hline 28 & Where am I? & $\begin{array}{l}\text { At home, at someone else's home, } \\
\text { work/school, public place, on my } \\
\text { way, somewhere else }\end{array}$ \\
\hline 29 & What am I doing (just before the beep)? & $\begin{array}{l}\text { Resting, work/school, household } \\
\text { work/shopping, hygiene/self-care, } \\
\text { eating/drinking, relaxing/recreation, } \\
\text { sports, travelling, something else }\end{array}$ \\
\hline 30 & I feel (un)comfortable doing this & $\begin{array}{l}-5 \text { (extremely uncomfortable) - } \\
+5 \text { (extremely comfortable) }\end{array}$ \\
\hline 31 & My symptoms are limiting my current activities & 0 (none) - 10 (very much) \\
\hline 32 & Who is with me? & $\begin{array}{l}\text { Partner/children, friends, } \\
\text { housemates, colleagues, family } \\
\text { (other than those who live in your } \\
\text { house), acquaintances, } \\
\text { strangers/others, no one }\end{array}$ \\
\hline 33 & I find this company (un)pleasant & $\begin{array}{l}-5 \text { (extremely unpleasant) }-+5 \\
\text { (extremely pleasant) }\end{array}$ \\
\hline
\end{tabular}


Table 3.3 Definitive set of items for the morning questionnaire for assessment once a day.

\begin{tabular}{|c|c|c|}
\hline & Morning questionnaire & Answer scale \\
\hline 1 & How long did it take you to fall asleep last night? & $\begin{array}{l}0-5 \text { min.; } 5-15 \text { min.; } 15-30 \text { min.; 30- } \\
45 \text { min.; } 45 \text { min.-1 hour; } 1-2 \text { hours; } 2- \\
4 \text { hours; more than } 4 \text { hours }\end{array}$ \\
\hline 2 & Last night, how many times did you wake up during the night? & $0,1,2,3,4,5$, more than 5 times \\
\hline 3 & I slept well & 0 (none) - 10 (very much) \\
\hline 4 & I feel rested & 0 (none) - 10 (very much) \\
\hline 5 & I had abdominal problems during the night & 0 (none) - 10 (very much) \\
\hline $5 a$ & During the night I suffered from: & $\begin{array}{l}\text { a full feeling, a heavy feeling, } \\
\text { bloating, pain in my upper abdomen, } \\
\text { a burning feeling in my upper } \\
\text { abdomen, pain in my lower } \\
\text { abdomen, nausea, vomiting, a } \\
\text { burning feeling behind the } \\
\text { breastbone, nocturnal defecation }\end{array}$ \\
\hline
\end{tabular}

\section{Discussion}

The current study was conducted in order to develop a novel PROM for assessment of symptoms in functional dyspepsia based on the Experience Sampling Method.

Development of the novel ESM-based PROM was executed according to FDA guidelines over four executive phases. A systematic literature search, patient focus group interviews, and international expert meeting led to a novel ESM-based PROM, containing 33 items. Interviews were performed to confirm patients' understanding, although adequate briefing before use of the novel PROM remains necessary as variable interpretations of the meaning of the terms nausea and bloating were reported during focus group interviews. In the future, addition of pictograms in the digital ESM-based PROM may be useful to improve comprehension of verbal symptom descriptors, as previously demonstrated by Tack et al. ${ }^{25}$ Moreover, forward-andbackward translation with additional cognitive interviews are necessary to verify patient understanding with the developed items in each language.

In line with the Rome IV criteria, the four core FD symptoms were described by the majority of patients. Additional frequently reported symptoms were bloating, nausea, and belching. These symptoms were also reported by a substantial subgroup of FD patients during previously performed focus group interviews, cognitive interviews, and validation studies (e.g. bloating $86-93 \%$, nausea $40-73 \%$, belching 27-69\%). ${ }^{26,27}$ Moreover, bloating was considered the most important symptom for improvement with effective therapy during development of the 'Functional Dyspepsia Symptom Diary'. ${ }^{27}$ As the FDA states that the effect of treatment should be measured at the level of each symptom in order to ensure that treatment does not negatively affect symptoms, we suggest that a PROM should, as a minimum, evaluate the four FD 
core symptoms as well as the three frequently occurring additional symptoms. ${ }^{8}$ Food intake, medication use, and psychosocial factors were considered important triggers for generation and/or severity of gastrointestinal symptoms, which is in line with previous studies. ${ }^{26,28-30}$ Repeated assessment of dyspeptic symptoms, together with symptom triggers, offers the potential to optimize and individualize treatment strategies, and this is a potential advantage of the novel ESM-based PROM, compared with the recently developed 'Leuven Postprandial Distress Scale' (LPDS) and 'Functional Dyspepsia Symptom Diary'. ${ }^{27,31}$

Besides dyspeptic symptoms, lower gastrointestinal symptoms were studied, as evidence has been found for an increased prevalence of IBS in patients with FD. ${ }^{3,32}$ In our patient focus groups, $21 \%$ of patients had a comorbid diagnosis of IBS according to the Rome IV criteria, and this subgroup recommended inclusion of questions with regard to lower abdominal pain and defecation pattern. Although we intended to develop a disease-specific PROM for functional dyspepsia, the substantial overlap with IBS and potential implications for individualized therapy lead to incorporation of IBS core symptoms (i.e. lower abdominal pain and defecation pattern) in the newly developed PROM.

Psychological factors such as anxiety and depression are associated with functional gastrointestinal disorders, and may precede or exacerbate symptoms. ${ }^{33-35}$ One of the main advantages of ESM compared with retrospective questionnaires is the fact that ESM offers the opportunity to improve ecological validity by taking into account these psychological factors. ${ }^{24}$ However, one point of discussion during the international expert meeting was the fact that no validated psychological ESM-items are currently available. In clinical trials, the Hospital Anxiety and Depression Scale (HADS) is often used to screen for the presence of anxiety or depressive disorders. However, HADS items are not eligible for momentary symptom assessment due to the retrospective nature. Verhagen et al. recently described psychometric validation properties of ESM in patients with mental disorders. Thirteen mood items were included in their ESM-questionnaire which could be divided into positive affect ( 4 items) and negative affect ( 9 items) based on factorial analysis. The authors demonstrated excellent reliability, and significant correlations were found between mean scores for positive and negative affect, and the HADS scores, demonstrating concurrent validity. ${ }^{24}$ However, inclusion of thirteen psychological items in an outcome measure for FD was considered too much of a burden, and we therefore reduced the psychological ESM-items from 13 to seven, in line with the previously developed ESM-IBS PROM. ${ }^{18}$ Additional research is necessary to validate these psychological ESM-items in patients with functional gastrointestinal disorders.

Somatization is also considered a factor contributing to symptom generation in functional gastrointestinal disorders. ${ }^{36-38}$ The Patient Health Questionnaire 12 (PHQ-12) is frequently used in clinical trials to assess somatic complaints. ${ }^{39}$ In the final ESM-FD PROM, five of the 12 items of the PHQ-12 were included because participants 
mentioned these complaints in focus group interviews (i.e. chest pain, dizziness, palpitations, dyspnea, and fatigue). The remaining seven items were not included, because they were not mentioned by focus group participants (back pain, pain in extremities or joints), they were mentioned by only one participant (headache, fainting), or the items were not eligible for assessment in a momentary questionnaire (sleeping problems).

Currently available outcome measures use several types of response options including Visual Analogue Scales (VAS), Likert scales, pictorial scales, and binary endpoints (adequate relief of symptoms: yes/no). ${ }^{25}$ Recently, our research group performed focus groups in IBS patients for the development of an ESM-based PROM, and discussed several endpoints including a 7-point NRS (formerly used in ESM) and 11-point NRS. ${ }^{18}$ Finally, the 11-point NRS scale was incorporated, as the use of an endof-day 11-point NRS is recommended by the FDA as primary endpoint in clinical trials of IBS patients. ${ }^{21}$ In contrast to IBS, a recommended response scale for FD is currently lacking. The 11-point NRS, which has been incorporated into the ESM-based IBSPROM, was discussed during the focus group meetings and was considered appropriate by FD patients. Therefore, the 11-point NRS was chosen, in order to have a uniform response option for ESM-based PROMs in functional gastrointestinal disorders.

Strengths of this study include the fact that the ESM-based PROM was developed according to steps recommended by the FDA, with development of a conceptual framework after an extensive literature search, organization of patient focus group interviews and an international expert meeting, and arrangement of cognitive interviews. ${ }^{8}$ Due to the extensive literature search, a broad spectrum of items was identified and only a few additional symptoms (e.g. headache, fainting) were mentioned by individual patients during the focus group interviews.

Several limitations should be noted. First, we intended to invite five to 10 participants per focus group in order to ensure active participation of patients and gain a variety of perspectives. Although the final sample of 14 participants could be considered relatively small, focus group interviews were continued until saturation of input was achieved. Moreover, comparable sample sizes have been described in previous studies. ${ }^{25,26}$ Second, there may be a risk of limited representativeness, as only $32 \%$ of the initially invited patients eventually participated, and all patients were secondary and tertiary care patients. One could hypothesize that more confident patients and/or patients with more severe symptoms may be more willing to participate in focus group meetings. However, we assume that the risk of altered representativeness is limited, due to the inclusion of patients with a variety of symptoms matching different FD subtypes, the extensive item selection, and the limited additional input of symptoms by patients during focus group interviews. Another limitation may reside in the distribution of FD subtypes among participants since only one patient with the FD-PDS subtype participated in patient focus group 
sessions, whereas during cognitive grasp interviews only one patient with the EPS subtype was included. Nevertheless, in total four patients with PDS (20\%), seven patients with EPS (35\%), and nine patients with PDS-EPS (45\%) were included. Therefore, we assume that the entire spectrum of dyspeptic symptoms was captured. $^{40-42}$ Participants did have to have sufficient command of the Dutch language, which means minority groups were underrepresented. Due to the inclusion of dyspeptic patients according to the Rome IV criteria, it is not possible to extrapolate this PROM to patients with a selected number of dyspeptic symptoms not fulfilling Rome criteria. Another potential limitation was the fact that during the cognitive interviews the draft of the PROM was presented on paper, as the digital application was not yet available.

Next step in the development of this novel ESM-based PROM is a validation study in patients with FD according to Rome IV criteria. In this study, psychometric validation properties (e.g. reliability, validity and responsiveness) will be assessed, and the final goal is to define a minimum clinically important difference for use in clinical trials. Another point of interest during this validation study is the compliance rate, as a subgroup of patients during the focus group interviews thought that ten assessments per day would be quite burdensome. However, an adequate compliance rate of $76.8 \%$ was found in a small pilot study in IBS patients using a 60-item ESM questionnaire. ${ }^{17}$

In conclusion, we report on the development of a digital disease-specific PROM for real-time assessment of symptoms in patients with FD using the ESM. Although future studies with this novel PROM are necessary to assess construct validity, reliability, and responsiveness, we suggest that the newly developed ESM-based PROM might be able to assess FD, identify specific triggers for symptoms, optimize (individualized) treatment strategies, and could potentially be used as an instrument to quantify therapeutic efficacy. 


\section{References}

1. Tack J, Talley NJ, Camilleri M, Holtmann G, Hu P, Malagelada JR, et al. Functional gastroduodenal disorders. Gastroenterology. 2006;130(5):1466-79.

2. Mahadeva S, Goh KL. Epidemiology of functional dyspepsia: a global perspective. World J Gastroenterol. 2006;12(17):2661-6.

3. Aziz I, Palsson OS, Tornblom H, Sperber AD, Whitehead WE, Simren M. Epidemiology, clinical characteristics, and associations for symptom-based Rome IV functional dyspepsia in adults in the USA, Canada, and the UK: a cross-sectional population-based study. Lancet Gastroenterol Hepatol. 2018;3(4):252-62.

4. Taylor F, Reasner DS, Carson RT, Deal LS, Foley C, lovin R, et al. Development of a Symptom-Based Patient-Reported Outcome Instrument for Functional Dyspepsia: A Preliminary Conceptual Model and an Evaluation of the Adequacy of Existing Instruments. Patient. 2016;9(5):409-18.

5. Stanghellini V, Chan FK, Hasler WL, Malagelada JR, Suzuki H, Tack J, et al. Gastroduodenal Disorders. Gastroenterology. 2016;150(6):1380-92.

6. Sander GB, Mazzoleni LE, Francesconi CF, Balbinotto G, Mazzoleni F, Wortmann AC, et al. Influence of organic and functional dyspepsia on work productivity: the HEROES-DIP study. Value Health. 2011;14(5 Suppl 1):S126-9.

7. Ford AC, Forman D, Bailey AG, Cook MB, Axon AT, Moayyedi P. Who consults with dyspepsia? Results from a longitudinal 10-yr follow-up study. Am J Gastroenterol. 2007;102(5):957-65.

8. Administration FaD. Guidance for Industry; Patient-Reported Outcome Measures: use in Medical Product Development to support Labeling Claims 2009.

9. Smeets FGM, Masclee AAM, Conchillo JM, Keszthelyi D. Systematic review: Disease-specific instruments to assess gastrointestinal symptoms in functional dyspepsia. Neurogastroenterol Motil. 2018.

10. van Os J, Verhagen S, Marsman A, Peeters F, Bak M, Marcelis M, et al. The experience sampling method as an mHealth tool to support self-monitoring, self-insight, and personalized health care in clinical practice. Depress Anxiety. 2017;34(6):481-93.

11. Feinle-Bisset C, Horowitz M. Dietary factors in functional dyspepsia. Neurogastroenterol Motil. 2006;18(8):608-18.

12. Mak AD, Wu JC, Chan Y, Chan FK, Sung JJ, Lee S. Dyspepsia is strongly associated with major depression and generalised anxiety disorder - a community study. Aliment Pharmacol Ther. 2012;36(8):800-10.

13. Shiffman S, Stone AA, Hufford MR. Ecological momentary assessment. Annu Rev Clin Psychol. 2008;4:1-32.

14. Stone AA, Shiffman S, Schwartz JE, Broderick JE, Hufford MR. Patient compliance with paper and electronic diaries. Control Clin Trials. 2003;24(2):182-99.

15. Myin-Germeys I, Oorschot M, Collip D, Lataster J, Delespaul P, van Os J. Experience sampling research in psychopathology: opening the black box of daily life. Psychol Med. 2009;39(9):1533-47.

16. Weinland SR, Morris CB, Hu Y, Leserman J, Bangdiwala SI, Drossman DA. Characterization of episodes of irritable bowel syndrome using ecological momentary assessment. Am J Gastroenterol. 2011;106(10):1813-20.

17. Mujagic Z, Leue C, Vork L, Lousberg R, Jonkers DM, Keszthelyi D, et al. The Experience Sampling Method--a new digital tool for momentary symptom assessment in IBS: an exploratory study. Neurogastroenterol Motil. 2015;27(9):1295-302.

18. Vork L, Keszthelyi D, Mujagic Z, Kruimel JW, Leue C, Ponten I, et al. Development, content validity, and cross-cultural adaptation of a patient-reported outcome measure for real-time symptom assessment in irritable bowel syndrome. Neurogastroenterol Motil. 2018;30(3).

19. Rabiee F. Focus-group interview and data analysis. Proc Nutr Soc. 2004;63(4):655-60.

20. Krueger RA. Designing and conducting focus group interviews. 2002.

21. (FDA) FaDA. Guidance for industry, Irritable bowel syndrome - Clinical evaluation of drugs for treatment 2012. 
22. Knafl K, Deatrick J, Gallo A, Holcombe G, Bakitas M, Dixon J, et al. The analysis and interpretation of cognitive interviews for instrument development. Res Nurs Health. 2007;30(2):224-34.

23. Krueger RA. Developing questions for focus groups. SAGA Publications. 1998.

24. Verhagen SJW, Berben JA, Leue C, Marsman A, Delespaul P, van Os J, et al. Demonstrating the reliability of transdiagnostic mHealth Routine Outcome Monitoring in mental health services using experience sampling technology. PLoS One. 2017;12(10):e0186294.

25. Tack J, Carbone F, Holvoet L, Vanheel H, Vanuytsel T, Vandenberghe A. The use of pictograms improves symptom evaluation by patients with functional dyspepsia. Aliment Pharmacol Ther. 2014;40(5):523-30.

26. Carbone F, Holvoet L, Vandenberghe A, Tack J. Functional dyspepsia: outcome of focus groups for the development of a questionnaire for symptom assessment in patients suffering from postprandial distress syndrome (PDS). Neurogastroenterol Motil. 2014;26(9):1266-74.

27. Taylor F, Higgins S, Carson RT, Eremenco S, Foley C, Lacy BE, et al. Development of a SymptomFocused Patient-Reported Outcome Measure for Functional Dyspepsia: The Functional Dyspepsia Symptom Diary (FDSD). Am J Gastroenterol. 2018;113(1):39-48.

28. Talley NJ. Functional dyspepsia: new insights into pathogenesis and therapy. Korean J Intern Med. 2016;31(3):444-56.

29. Fang YJ, Liou JM, Chen CC, Lee JY, Hsu YC, Chen MJ, et al. Distinct aetiopathogenesis in subgroups of functional dyspepsia according to the Rome III criteria. Gut. 2015;64(10):1517-28.

30. Bisschops R, Karamanolis G, Arts J, Caenepeel P, Verbeke K, Janssens J, et al. Relationship between symptoms and ingestion of a meal in functional dyspepsia. Gut. 2008;57(11):1495-503.

31. Carbone F, Vandenberghe A, Holvoet L, Vanuytsel T, Van Oudenhove L, Jones $M$, et al. Validation of the Leuven Postprandial Distress Scale, a questionnaire for symptom assessment in the functional dyspepsia/postprandial distress syndrome. Aliment Pharmacol Ther. 2016;44(9):989-1001.

32. Ford AC, Marwaha A, Lim A, Moayyedi P. Systematic review and meta-analysis of the prevalence of irritable bowel syndrome in individuals with dyspepsia. Clin Gastroenterol Hepatol. 2010;8(5):401-9.

33. Wouters MM, Boeckxstaens GE. Is there a causal link between psychological disorders and functional gastrointestinal disorders? Expert Rev Gastroenterol Hepatol. 2016;10(1):5-8.

34. Aro P, Talley NJ, Johansson SE, Agreus L, Ronkainen J. Anxiety Is Linked to New-Onset Dyspepsia in the Swedish Population: A 10-Year Follow-up Study. Gastroenterology. 2015;148(5):928-37.

35. Pinto-Sanchez MI, Ford AC, Avila CA, Verdu EF, Collins SM, Morgan D, et al. Anxiety and Depression Increase in a Stepwise Manner in Parallel With Multiple FGIDs and Symptom Severity and Frequency. Am J Gastroenterol. 2015;110(7):1038-48.

36. Van Oudenhove L, Vandenberghe J, Geeraerts B, Vos R, Persoons P, Fischler B, et al. Determinants of symptoms in functional dyspepsia: gastric sensorimotor function, psychosocial factors or somatisation? Gut. 2008;57(12):1666-73.

37. Simren M, Tornblom H, Palsson OS, van Tilburg MAL, Van Oudenhove L, Tack J, et al. Visceral hypersensitivity is associated with GI symptom severity in functional GI disorders: consistent findings from five different patient cohorts. Gut. 2018;67(2):255-62.

38. Gracie DJ, Bercik P, Morgan DG, Bolino C, Pintos-Sanchez MI, Moayyedi P, et al. No increase in prevalence of somatization in functional vs organic dyspepsia: a cross-sectional survey. Neurogastroenterol Motil. 2015;27(7):1024-31.

39. Spiller RC, Humes DJ, Campbell E, Hastings M, Neal KR, Dukes GE, et al. The Patient Health Questionnaire 12 Somatic Symptom scale as a predictor of symptom severity and consulting behaviour in patients with irritable bowel syndrome and symptomatic diverticular disease. Aliment Pharmacol Ther. 2010;32(6):811-20.

40. Vakil N, Halling K, Ohlsson L, Wernersson B. Symptom overlap between postprandial distress and epigastric pain syndromes of the Rome III dyspepsia classification. Am J Gastroenterol. 2013;108(5):767-74.

41. Carbone F, Holvoet L, Tack J. Rome III functional dyspepsia subdivision in PDS and EPS: recognizing postprandial symptoms reduces overlap. Neurogastroenterol Motil. 2015;27(8):1069-74.

42. Carbone F, Holvoet L, Vanuytsel T, Tack J. Rome III functional dyspepsia symptoms classification: Severity vs frequency. Neurogastroenterol Motil. 2017;29(6). 



\section{Part II}

Prediction of treatment outcome in upper gastrointestinal disorders 


\section{Chapter 4}

Esophagogastric junction distensibility in the management of achalasia patients: relation to treatment outcome

F.G.M. Smeets, A.A.M. Masclee, D. Keszthelyi, E.T.T.L. Tjwa, J.M. Conchillo Neurogastroenterol Motil. 2015;27(10):1495-1503 


\section{Abstract}

\section{Background}

Achalasia is characterized by a functional obstruction at the esophagogastric junction (EGJ). The functional luminal imaging probe (EndoFLIP) is a new method to assess EGJ distensibility. In a homogeneous group of newly diagnosed achalasia patients treated with pneumatic dilation (PD), we aimed (a) to determine whether assessment of EGJ distensibility has added value in the management of achalasia patients and (b) to evaluate whether EGJ distensibility differs between achalasia subtypes.

\section{Methods}

Twenty-six newly diagnosed achalasia patients were treated by graded PD (30-mm and $35-\mathrm{mm}$ ) separated by one week. EGJ distensibility was measured with the EndoFLIP technique before and after 30-mm PD. Good clinical treatment outcome was defined as an Eckardt score $<4$ at one-year follow-up. Fifteen healthy controls underwent an EndoFLIP measurement as control group.

\section{Key results}

Newly diagnosed achalasia patients had reduced EGJ distensibility compared to healthy controls $\left(0.9(0.7-1.5)\right.$ vs. $\left.3.4(2.7-4.2) \mathrm{mm}^{2} / \mathrm{mmHg}, \mathrm{p}<0.01\right)$, and EGJ distensibility was lower in type II compared to type I patients $(0.8$ (0.7-1.1) vs. $\left.1.5(0.9-1.9) \mathrm{mm}^{2} / \mathrm{mmHg}, \mathrm{p}=0.02\right)$. EGJ distensibility was increased after PD from $0.9(0.7-1.5)$ to $4.2(3.0-5.7) \mathrm{mm}^{2} / \mathrm{mmHg}(p<0.001)$. No difference was found in EGJ distensibility directly after PD between patients with good and poor clinical outcome at one-year follow-up.

\section{Conclusions \& Inferences}

Assessment of EGJ distensibility with the EndoFLIP technique is able to demonstrate the functional EGJ obstruction in newly diagnosed achalasia patients and EGJ distensibility differs between achalasia subtypes. Although PD improves EGJ distensibility, assessment of EGJ distensibility with a limited number of distension steps provides no additional information that is useful for clinical evaluation and management of achalasia patients. 


\section{Introduction}

Achalasia is a primary esophageal motility disorder associated with functional obstruction at the level of the esophagogastric junction (EGJ) due to defective relaxation of the lower esophageal sphincter (LES). ${ }^{1-3}$ Until recently, it was not possible to measure direct EGJ opening or EGJ distensibility. High-resolution manometry and timed barium esophagogram measure surrogate parameters of EGJ opening, i.e. deglutitive EGJ relaxation pressure and bolus retention. Recently, an Endoscopic Functional Luminal Imaging Probe (EndoFLIP) has been developed to assess EGJ distensibility during volumetric balloon distensions using impedance planimetry. ${ }^{4-7}$ Recent studies using the EndoFLIP technique have described a reduced EGJ distensibility in newly diagnosed achalasia patients compared to healthy controls. ${ }^{8,9}$ Achalasia patients can be categorized into three manometric subtypes according to the Chicago classification. ${ }^{10}$ Previous studies found differences in LES resting pressure and integrated relaxation pressure between achalasia subtypes, probably due to different patterns of neuronal function. ${ }^{10-12}$ Here, we hypothesize that EGJ distensibility might differ between achalasia subtypes and that assessment of EGJ distensibility with the EndoFLIP technique might contribute to a better understanding of achalasia subtypes.

Treatment of achalasia is focused on improving bolus transport across the EGJ through either (graded) pneumatic balloon dilation or surgical myotomy. ${ }^{13-15}$ In two cross-sectional studies, achalasia patients with a good clinical outcome after treatment had a higher EGJ distensibility compared to patients with poor clinical outcome. ${ }^{8,9}$ Moreover, recent studies described an increased EGJ distensibility either immediately post procedure or three months after treatment with pneumatic dilation, laparoscopic Heller myotomy (LHM) or peroral endoscopic myotomy (POEM)., ${ }^{9,16-18}$ In addition, intraoperative EGJ distensibility was predictive of postoperative symptomatic outcome after surgical myotomy. ${ }^{18}$ However, data are lacking about the relation between EGJ distensibility after treatment with pneumatic balloon dilation and mid or long-term clinical outcome. Here, we hypothesize that EGJ distensibility as measured by EndoFLIP is a good indicator or predictor of clinical treatment outcome after pneumatic balloon dilation and that EGJ distensibility may differ between achalasia subtypes. In a homogeneous group of newly diagnosed achalasia patients treated with pneumatic balloon dilation, we therefore aimed (a) to determine whether assessment of EGJ distensibility has added value in the management of achalasia patients, and (b) to evaluate whether EGJ distensibility differs between achalasia subtypes. 


\section{Materials and methods}

\section{Subjects}

Twenty-six newly diagnosed achalasia patients were recruited from our outpatient clinic to participate in the present study. All patients had a clinical presentation of achalasia with an Eckardt score $>3$ and the diagnosis was confirmed on esophageal manometry based on the absence of peristalsis and impaired EGJ relaxation (mean integrated relaxation pressure (IRP) $>15 \mathrm{mmHg}$ on high resolution manometry). ${ }^{19}$ Patients with previous achalasia treatment and either esophageal or gastric surgery were excluded. Fifteen healthy controls underwent EndoFLIP measurement and were utilized as a control group for EGJ distensibility. Achalasia patients underwent graded pneumatic balloon dilation and EndoFLIP measurement as regular patient care and gave consent for publication of the data. The protocol for the healthy controls was approved by the medical ethical committee of the Maastricht University Medical Center (METC 13-2-037, NL45176.068.13) and was registered (ClinicalTrials.gov, Number NCT01956877).

\section{Study design}

Healthy controls underwent upper gastrointestinal endoscopy to assess the EGJ and place the EndoFLIP probe across the EGJ. After correct placement of the EndoFLIP probe, the endoscope was removed and volumetric distension of the EndoFLIP balloon was started. Patients with a clinical presentation of achalasia underwent esophageal manometry to confirm the diagnosis and graded pneumatic balloon dilation was performed using Rigiflex $30-\mathrm{mm}$ and $35-\mathrm{mm}$ balloons separated by one week. Distensibility of the EGJ was determined with the EndoFLIP technique before and after the Rigiflex 30-mm pneumatic balloon dilation. Esophageal manometry was repeated one month after treatment, whereas clinical outcome was assessed at one month and one-year follow-up using the Eckardt questionnaire (Figure 4.1).

\section{Assessment of EGJ distensibility using EndoFLIP}

Esophagogastric junction distensibility was measured using a commercially available functional luminal imaging probe (EndoFLIP; Crospon Medical Devices, Galway, Ireland), as previously described. ${ }^{4,6}$ In brief, the EndoFLIP probe (EF-325N) consists of a catheter with a $14 \mathrm{~cm}$ noncompliant bag, which uses impedance planimetry to determine cross sectional areas (CSAs) for 16 balloon cross sections during volumetric distensions. Intra-bag pressure is determined by means of one pressure sensor inside the distal end of the EndoFLIP balloon allowing assessment of EGJ distensibility of the distended area. Prior to insertion of the catheter baseline pressure was zeroed. 
In healthy controls, upper endoscopy with EndoFLIP measurement was performed without sedation, whereas achalasia patients received moderate conscious sedation under cardiopulmonary monitoring since they were subsequently treated by pneumatic balloon dilation. At the end of the endoscopy, the deflated EndoFLIP balloon was inserted transorally and placed across the EGJ. After proper placement of the balloon, the endoscope was removed and the EndoFLIP balloon was inflated to 30 , 40 and $50 \mathrm{ml}$ distension volumes. At each distension volume, a 30 second measurement was made to determine CSAs, intra-bag pressure and EGJ distensibility. An hourglass shape of the balloon was observed in all measurements with the narrowest CSA at the level of the EGJ. Measurements interrupted by migration of the balloon were repeated. After completing the measurements, the EndoFLIP balloon was deflated and removed.

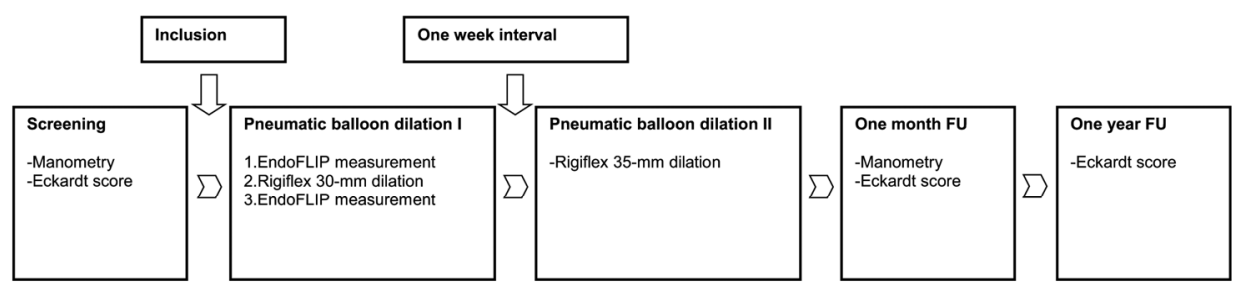

Figure 4.1 Protocol for newly diagnosed achalasia patients. FU = follow-up.

\section{Pneumatic balloon dilation}

Pneumatic balloon dilation was performed using the Rigiflex balloon (Boston Scientific, Marlborough, USA). After the first EndoFLIP measurement, the Rigiflex 30-mm balloon was positioned across the EGJ and dilated at a pressure of 5 PSI for 3 minutes. After the Rigiflex $30-\mathrm{mm}$ dilation, the EndoFLIP probe was reintroduced and the procedure as previously described was repeated. One week later, the pneumatic dilation procedure was repeated with the Rigiflex $35-\mathrm{mm}$ balloon in all patients.

\section{Esophageal manometry}

High resolution manometry (HRM) was performed prior to treatment and at one month follow-up using a solid-state HRM catheter (Unisensor AG, Attikon, Switzerland) containing 36 circumferential pressure sensors spaced at $1 \mathrm{~cm}$ intervals with a maximum outer diameter of $3.3 \mathrm{~mm}$. Catheter position was considered correct when pressure recordings from the hypopharynx to the stomach were present with at 
least two sensors located in the stomach. EGJ resting pressure was measured and ten wet swallows $(5 \mathrm{ml}$ ) were used to assess integrated relaxation pressure (IRP).

\section{Questionnaire}

Symptoms were assessed using the Eckardt questionnaire consisting of four questions measuring dysphagia, chest pain, weight loss and regurgitation with scores from 0 (no symptoms) to 3 (incapacitating symptoms). ${ }^{20}$ Total Eckardt scores were calculated by summing the individual items with a score ranging from 0 to 12 . Symptoms were evaluated prior to treatment, and clinical outcome was assessed at one month and one-year follow-up. Treatment was considered successful if the Eckardt score was below 4 .

\section{Data analysis}

Esophagogastric junction distensibility was assessed using the mean value over a 30 seconds dynamic measurement of the narrowest CSA and the corresponding intrabag pressure (narrowest CSA in $\mathrm{mm}^{2}$ /intra-bag pressure in $\mathrm{mmHg}$ ). ${ }^{4}$

High-resolution manometry studies were analysed using MMS analysis software to measure integrated relaxation pressure (IRP), which quantifies completeness and persistence of EGJ relaxation. The upper limit of normal for IRP was $<15 \mathrm{mmHg} .{ }^{19}$ Achalasia patients were divided into three subtypes according to the Chicago classification. Type I patients had no distal esophageal pressurization to $>30 \mathrm{mmHg}$ in at least 8 of the 10 test swallows. In type II patients, at least 2 test swallows were associated with panesophageal pressurization $>30 \mathrm{mmHg}$, whereas type III patients had $\geq 2$ spastic contractions with or without periods of compartmentalized pressurization. $^{12}$

\section{Statistical analysis}

All statistical analyses were performed using commercially available computer software (PASW Statistics for Windows, Release Version 20.0.0, C SPSS, Inc, Chicago, IL, USA). Continuous data were expressed as median (interquartile range, IQR). Paired data were tested using the Wilcoxon signed rank test and Mann Whitney $U$ test was used to compare unpaired data. Correlations between continuous variables were calculated using Spearman correlation. Values of $p<0.05$ were considered significant. 


\section{Results}

\section{Subjects}

Fifteen healthy controls (5 males, median age 23 years, range $19-50$ years) and 26 newly diagnosed achalasia patients (14 males, median age 52 years, range 19-75 years) participated in the study. Median age was higher in achalasia patients compared to healthy controls $(p<0.01)$, whereas gender distribution was similar between groups.

\section{Healthy controls versus newly diagnosed achalasia patients}

Healthy controls had a median EGJ distensibility of $3.0(2.2-4.2) \mathrm{mm}^{2} / \mathrm{mmHg}$ and 3.4 $(2.7-4.2) \mathrm{mm}^{2} / \mathrm{mmHg}$ at the $40 \mathrm{ml}$ and $50 \mathrm{ml}$ volumetric distension volumes, respectively. Newly diagnosed achalasia patients had a lower EGJ distensibility compared to healthy controls at all distension volumes (Table 4.1, Figure 4.2). The $10^{\text {th }}$ percentile of EGJ distensibility in healthy controls was assessed to determine a cutoff value for normality, which was $2.1 \mathrm{~mm}^{2} / \mathrm{mmHg}$ at the $50 \mathrm{ml}$ distension volume. Ninety-six percent of the newly diagnosed achalasia patients had an EGJ distensibility below this cut-off value prior to treatment.

Table 4.1 Comparison of esophagogastric junction (EGJ) distensibility between newly diagnosed achalasia patients and healthy controls during volumetric balloon distensions with the EndoFLIP technique.

\begin{tabular}{|c|c|c|c|}
\hline & $\begin{array}{l}\text { Newly diagnosed } \\
\text { achalasia patients } \\
(n=26)\end{array}$ & $\begin{array}{l}\text { Healthy controls } \\
\qquad(n=15)\end{array}$ & p-value \\
\hline EGJ distensibility $30 \mathrm{ml}\left(\mathrm{mm}^{2} / \mathrm{mmHg}\right)$ & $1.2(0.8-1.6)$ & $2.0(1.6-3.0)$ & 0.01 \\
\hline - Pressure $(\mathrm{mmHg})$ & $17.6(12.7-26.6)$ & $25.9(21.4-30.0)$ & 0.03 \\
\hline - CSA $\left(\mathrm{mm}^{2}\right)$ & $20.2(19.9-20.7)$ & $50.4(41.7-69.1)$ & $<0.01$ \\
\hline EGJ distensibility $40 \mathrm{ml}\left(\mathrm{mm}^{2} / \mathrm{mmHg}\right)$ & $0.8(0.7-1.4)$ & $3.0(2.2-4.2)$ & $<0.01$ \\
\hline - Pressure $(\mathrm{mmHg})$ & $26.5(21.1-36.1)$ & $34.5(29.0-40.2)$ & 0.02 \\
\hline - CSA $\left(\mathrm{mm}^{2}\right)$ & $24.1(20.1-31.6)$ & $99.5(79.5-138.0)$ & $<0.01$ \\
\hline EGJ distensibility $50 \mathrm{ml}\left(\mathrm{mm}^{2} / \mathrm{mmHg}\right)$ & $0.9(0.7-1.5)$ & $3.4(2.7-4.2)$ & $<0.01$ \\
\hline - Pressure $(\mathrm{mmHg})$ & $46.8(42.2-57.1)$ & $45.8(41.9-53.4)$ & 0.56 \\
\hline$-\operatorname{CSA}\left(\mathrm{mm}^{2}\right)$ & $47.6(40.4-65.1)$ & $169.4(131.7-187.9)$ & $<0.01$ \\
\hline
\end{tabular}

\section{Achalasia subtypes}

Patients were divided into achalasia subtypes according to the Chicago classification. Nine patients were diagnosed with type I (35\%) and 17 patients (65\%) had type II achalasia. Patients with type II achalasia had a lower EGJ distensibility before treatment compared to type I achalasia patients (Table 4.2). Resting pressure of EGJ was higher in type II compared to type I patients (49 (38-72) vs. 34 (23-46) mmHg, 
$p<0.05)$ with no difference in IRP. No differences were found in EGJ distensibility, EGJ resting pressure or IRP between achalasia subtypes after pneumatic dilation.

A Healthy control

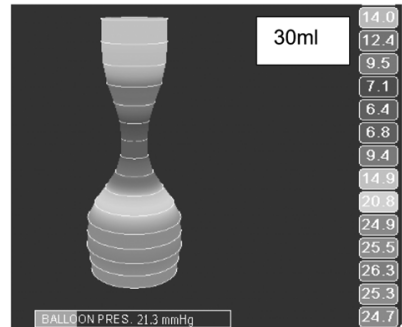

EGJ distensibility $=1.5 \mathrm{~mm}^{2} / \mathrm{mmHg}$ $\mathrm{CSA}=32 \mathrm{~mm}^{2}$

Pressure $=21.3 \mathrm{mmHg}$

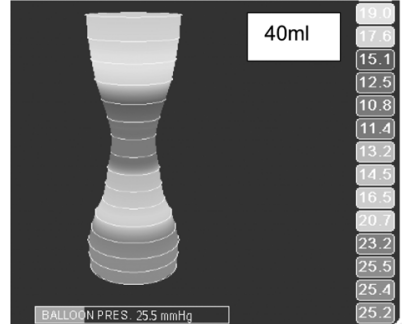

EGJ distensibility $=3.6 \mathrm{~mm}^{2} / \mathrm{mmHg}$ $\mathrm{CSA}=92 \mathrm{~mm}^{2}$

Pressure $=25.5 \mathrm{mmHg}$

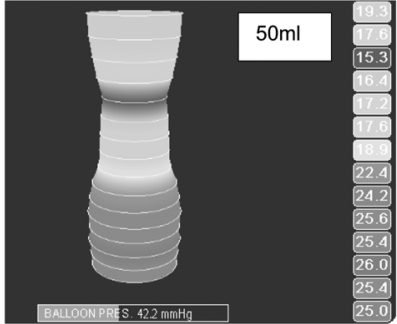

EGJ distensibility $=4.4 \mathrm{~mm}^{2} / \mathrm{mmHg}$ $\mathrm{CSA}=184 \mathrm{~mm}^{2}$

Pressure $=42.2 \mathrm{mmHg}$

B Newly diagnosed achalasia patient

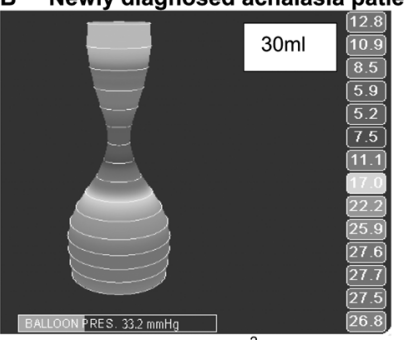

EGJ distensibility $=0.6 \mathrm{~mm}^{2} / \mathrm{mmHg}$ $\mathrm{CSA}=21 \mathrm{~mm}^{2}$

Pressure $=33.2 \mathrm{mmHg}$

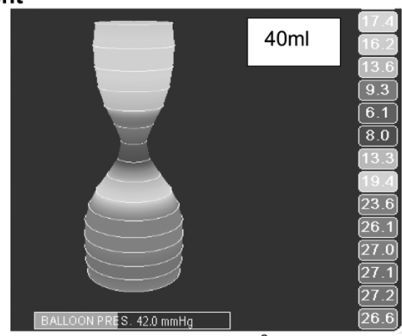

EGJ distensibility $=0.7 \mathrm{~mm}^{2} / \mathrm{mmHg}$ CSA $=29 \mathrm{~mm}^{2}$

Pressure $=42.0 \mathrm{mmHg}$

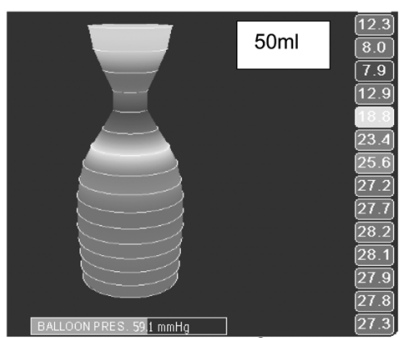

EGJ distensibility $=0.8 \mathrm{~mm}^{2} / \mathrm{mmHg}$ $\mathrm{CSA}=49 \mathrm{~mm}^{2}$

Pressure $=59.1 \mathrm{mmHg}$

Figure 4.2 Examples of EndoFLIP measurements during volumetric distensions in $(A)$ a healthy control and (B) a newly diagnosed achalasia patient demonstrating different EGJ distensibility patterns. Increasing volumetric distension of the EndoFLIP balloon results in an increase in both EGJ opening and EGJ distensibility in healthy controls, whereas EGJ opening and EGJ distensibility do not markedly increase in newly diagnosed achalasia patients.

Table 4.2 Comparison of esophagogastric junction (EGJ) distensibility between achalasia subtypes I and II during volumetric balloon distensions with the EndoFLIP technique.

\begin{tabular}{|c|c|c|c|}
\hline & $\begin{array}{c}\text { Achalasia subtype I } \\
\qquad(n=9)\end{array}$ & $\begin{array}{c}\text { Achalasia subtype II } \\
\qquad(n=17)\end{array}$ & p-value \\
\hline EGJ distensibility $30 \mathrm{ml}\left(\mathrm{mm}^{2} / \mathrm{mmHg}\right)$ & $1.3(1.2-1.9)$ & $1.0(0.7-1.3)$ & $<0.05$ \\
\hline - Pressure (mmHg) & $15.6(11.3-22.1)$ & $20.2(15.6-28.1)$ & 0.14 \\
\hline$-\mathrm{CSA}\left(\mathrm{mm}^{2}\right)$ & $20.5(20.0-21.3)$ & $20.1(19.8-20.5)$ & $<0.05$ \\
\hline EGJ distensibility $40 \mathrm{ml}\left(\mathrm{mm}^{2} / \mathrm{mmHg}\right)$ & $1.1(0.8-1.7)$ & $0.8(0.5-1.1)$ & 0.06 \\
\hline - Pressure $(\mathrm{mmHg})$ & $21.0(19.4-30.9)$ & $27.6(23.4-49.1)$ & $<0.05$ \\
\hline$-\operatorname{CSA}\left(\mathrm{mm}^{2}\right)$ & $26.9(21.1-34.5)$ & $22.7(19.9-29.9)$ & 0.25 \\
\hline EGJ distensibility $50 \mathrm{ml}\left(\mathrm{mm}^{2} / \mathrm{mmHg}\right)$ & $1.5(0.9-1.9)$ & $0.8(0.7-1.1)$ & $<0.05$ \\
\hline - Pressure (mmHg) & $40.5(38.5-48.4)$ & $49.8(45.8-66.6)$ & $<0.05$ \\
\hline$-\operatorname{CSA}\left(\mathrm{mm}^{2}\right)$ & $61.9(45.5-80.9)$ & $44.2(35.3-53.9)$ & 0.07 \\
\hline
\end{tabular}




\section{Effect of pneumatic balloon dilation on EGJ distensibility}

Treatment of newly diagnosed achalasia patients by single pneumatic balloon dilation (Rigiflex 30-mm) improved EGJ distensibility at all volumetric distension volumes (Table 4.3). One month after graded pneumatic balloon dilation, symptoms were improved with a decline in Eckardt score from $7(6-8)$ to $0(0-1)(p<0.001)$ and 22 patients (85\%) had a good clinical outcome (i.e. Eckardt score <4). Distensibility of the EGJ increased above the $2.1 \mathrm{~mm}^{2} / \mathrm{mmHg}$ cutoff value after single pneumatic dilation in $95 \%$ of patients with a good clinical outcome but also in $75 \%$ of patients with a poor clinical outcome. No correlation was found between the increase in EGJ distensibility after the $30-\mathrm{mm}$ dilation and the improvement in symptom scores at one month follow-up.

Table 4.3 Effect of single pneumatic balloon dilation (30-mm) on esophagogastric junction (EGJ) distensibility in newly diagnosed achalasia patients $(n=26)$.

\begin{tabular}{|c|c|c|c|}
\hline & $\begin{array}{c}\text { Before pneumatic } \\
\text { balloon dilation (30-mm) }\end{array}$ & $\begin{array}{l}\text { After pneumatic balloon } \\
\text { dilation }(30-\mathrm{mm})\end{array}$ & p-value \\
\hline EGJ distensibility $30 \mathrm{ml}\left(\mathrm{mm}^{2} / \mathrm{mmHg}\right)$ & $1.2(0.8-1.6)$ & $1.6(1.4-2.2)$ & $<0.01$ \\
\hline - Pressure $(\mathrm{mmHg})$ & $17.6(12.7-26.6)$ & $16.2(13.9-25.5)$ & 0.14 \\
\hline$-\mathrm{CSA}\left(\mathrm{mm}^{2}\right)$ & $20.2(19.9-20.7)$ & $28.7(21.1-42.7)$ & $<0.01$ \\
\hline EGJ distensibility $40 \mathrm{ml}\left(\mathrm{mm}^{2} / \mathrm{mmHg}\right)$ & $0.8(0.7-1.4)$ & $3.2(2.2-4.0)$ & $<0.01$ \\
\hline - Pressure $(\mathrm{mmHg})$ & $26.5(21.1-36.1)$ & $24.0(19.8-30.3)$ & 0.01 \\
\hline$-\mathrm{CSA}\left(\mathrm{mm}^{2}\right)$ & $24.1(20.1-31.6)$ & $77.6(55.0-104.3)$ & $<0.01$ \\
\hline EGJ distensibility $50 \mathrm{ml}\left(\mathrm{mm}^{2} / \mathrm{mmHg}\right)$ & $0.9(0.7-1.5)$ & $4.2(3.0-5.7)$ & $<0.01$ \\
\hline - Pressure (mmHg) & $46.8(42.2-57.1)$ & $39.1(31.4-45.0)$ & $<0.01$ \\
\hline$-\operatorname{CSA}\left(\mathrm{mm}^{2}\right)$ & $47.6(40.4-65.1)$ & $173.0(112.6-206.3)$ & $<0.01$ \\
\hline
\end{tabular}

\section{Esophageal manometry}

Treatment significantly decreased mean EGJ resting pressure (from 43 (27-53) to $26(16-41) \mathrm{mmHg}, \mathrm{p}=0.001$ ) and IRP (from $26(19-40)$ to $14(10-16) \mathrm{mmHg}, \mathrm{p}<0.001$ ) at one month follow-up. No correlation was found between EGJ distensibility after the 30-mm dilation and mean EGJ resting pressure or IRP at one month follow-up ( $R=-$ $0.27, p=0.26$ and $R=-0.36, p=0.13$, respectively).

\section{Clinical outcome at one-year follow-up}

Clinical outcome at one-year follow-up was available for 22 patients (85\%) since the remaining patients were treated less than one year ago. Sixteen patients (73\%) had a good clinical outcome whereas all patients with poor clinical outcome underwent additional pneumatic dilation procedures. Three patients with treatment failure had elevated IRP at one month follow up. No differences were found in EGJ distensibility both before and immediately after pneumatic balloon dilation, between patients with a good or poor clinical outcome at one-year follow-up. Moreover, no correlation was 
found between EGJ distensibility before or after treatment, and clinical outcome at both one month and one-year follow-up. Only one out of six patients with a poor clinical treatment outcome had an abnormal EGJ distensibility (below $10^{\text {th }}$ percentile) directly after the 30-mm pneumatic dilation (Figure 4.3). Thus, the other five patients with a poor clinical response all had post-procedure EGJ distensibility in the normal range.

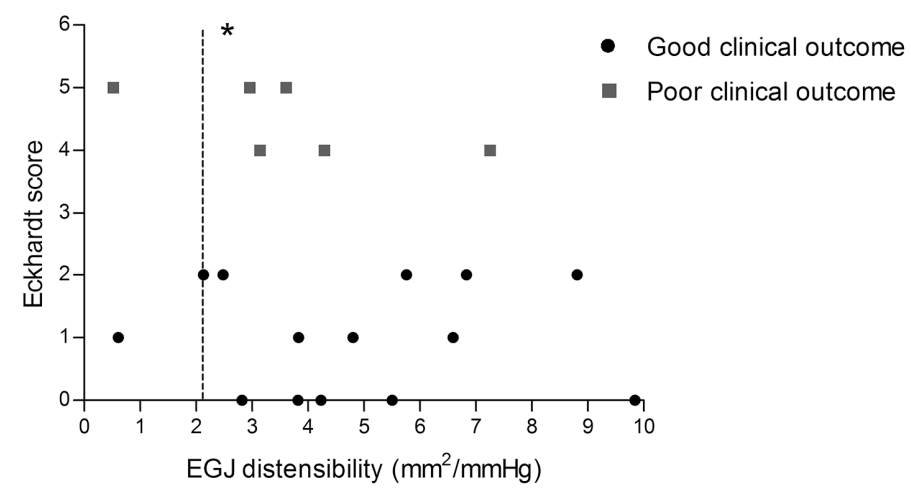

Figure 4.3 Distensibility of the esophagogastric junction directly after $30-\mathrm{mm}$ pneumatic dilation in patients with a good and poor clinical outcome at one-year follow-up. * Dotted line represents cutoff value for normality $\left(2.1 \mathrm{~mm}^{2} / \mathrm{mmHg}\right)$.

\section{Discussion}

In this study, we describe reduced distensibility of the esophagogastric junction (EGJ) in newly diagnosed achalasia patients compared to healthy controls, with type II achalasia patients showing lower EGJ distensibility compared to patients with type I achalasia. Although treatment by pneumatic balloon dilation immediately increased EGJ distensibility, post dilation EGJ distensibility was not related to clinical treatment outcome.

Achalasia is characterized by a functional obstruction at the level of the EGJ. The EndoFLIP technique was able to demonstrate this obstruction: $96 \%$ of the untreated achalasia patients had an EGJ distensibility below the cutoff value determined in healthy controls. Moreover, achalasia patients had a characteristic EGJ distensibility pattern, different from controls. No increase in distensibility was observed with increasing distension volumes of the EndoFLIP balloon, in contrast to the controls where an increase in distensibility during higher distension volumes was noted. ${ }^{4,9}$

In addition to the difference in EGJ distensibility patterns between newly diagnosed achalasia patients and healthy controls, a difference in EGJ distensibility 
was found between achalasia subtypes. Type II achalasia patients showed a lower EGJ distensibility and a higher EGJ resting pressure compared to type I patients, and this is also observed in a recent published study. ${ }^{18}$ We hypothesize that this difference in EGJ distensibility is related to the various stages of the disease. Achalasia is considered an autoimmune disease in which the type and intensity of the immune response probably contribute to the progression of neuronal loss. ${ }^{10}$ Chronic inflammation with progressive plexopathy may cause progression from type II to type I achalasia with differences in neuronal function at the level of the LES resulting in disturbed inhibitory and excitatory innervation of the LES and smooth muscles in the distal esophagus. Some preserved excitatory innervation to both LES and longitudinal muscles in the distal esophagus might explain the higher EGJ resting pressure and decreased EGJ distensibility in type II patients compared to type I patients, although conflicting results regarding manometry data have been described in achalasia subtypes. ${ }^{10-12}$

Current treatment options for achalasia are focused on relieving the functional obstruction at the level of the EGJ., $8,16,20$ Treatment of newly diagnosed achalasia patients by one single $(30-\mathrm{mm})$ pneumatic balloon dilation resulted in an increase in EGJ distensibility directly after the procedure. Our findings are in line with previous studies in which improved EGJ distensibility was observed direct post-procedure or three months after treatment with either pneumatic balloon dilation, laparoscopic Heller myotomy or POEM. ${ }^{9,16-18}$ Rohof et al. found a correlation between the increase in EGJ distensibility and the improvement of achalasia symptoms three months after treatment in 7 newly diagnosed achalasia patients. ${ }^{9}$ In contrast to this study, we did not find a correlation between the increase in EGJ distensibility directly after the 30-mm dilation and the improvement in symptom scores at both one month and oneyear follow-up. The reason for this discrepancy in results is not obvious, but several factors may be involved. First, direct assessment of EGJ distensibility after the $30-\mathrm{mm}$ dilation might be a potential reason for the lack of correlation. Edema might be present as a consequence of the dilation procedure resulting in an underestimation of EGJ distensibility immediately after the procedure. ${ }^{24}$ In addition, we hypothesized that assessment of EGJ distensibility directly after graded pneumatic dilation might demonstrate an additional increase in EGJ distensibility. However, no information is available about the influence of graded pneumatic dilation on EGJ distensibility and additional research is necessary. Second, there are differences in treatment protocols between studies with regard to pneumatic balloon dilation (e.g. duration of dilation and pressure used) which might influence the results. ${ }^{9}$ Moreover, treatment outcome is influenced by achalasia subtypes and we cannot exclude that a difference in the distribution of patient subtypes may have contributed to the discrepancy in results. ${ }^{21}$ In this study, the sample size was too small to determine the association between achalasia subtypes and clinical outcome. Previous studies described higher success rates of treatment for patients with type II achalasia compared to other achalasia subtypes. $^{12,21,23}$ In this study, post-dilation EGJ distensibility did not differ between 
achalasia subtypes. Therefore, the combination of both a reduction in EGJ obstruction after treatment and presence of panesophageal pressurization in type II patients may account for better clinical outcome in these patients compared to other achalasia subtypes. ${ }^{21,23,25}$

Clinical outcome at one-year follow-up was available for 22 patients and six patients were considered treatment failures. We hypothesized that EGJ distensibility assessed before or immediately after $30-\mathrm{mm}$ pneumatic dilation is able to predict clinical treatment outcome. However, no correlation was found between EGJ distensibility either before or after pneumatic dilation and clinical outcome at oneyear follow-up. Moreover, only one out of six patients with treatment failure had an EGJ distensibility below the cutoff value of normality after the $30-\mathrm{mm}$ pneumatic dilation, the other five patients who failed to respond had EGJ distensibility in the normal range. Although we have to consider the small number of patients and potential type II error, our data indicate that EGJ distensibility fails to predict clinical outcome in newly diagnosed achalasia patients treated by a standardized pneumatic dilation protocol.

Measurement of EGJ distensibility with the EndoFLIP technique is a new method and the exact role and relevance of this technique in the diagnosis and treatment of achalasia patients still has to be determined. In the present study, the EndoFLIP technique was used to provide additional information about EGJ distensibility in the management of achalasia patients. Esophageal manometry remains, however, the preferred test to diagnose achalasia and to divide patients into subtypes for several reasons. First, manometry provides information about both EGJ relaxation and esophageal contractility and is available in a large number of centers. ${ }^{8}$ Moreover, it is difficult to compare EndoFLIP data between centers due to the variety of results described in previous studies. ${ }^{4,18,26}$ Earlier studies suggested that improvement of EGJ distensibility was associated with treatment success, and a recent study described that the EndoFLIP technique might have a potential role for myotomy calibration during LHM and POEM. ${ }^{18}$ However, data on clinical follow-up after treatment with pneumatic balloon dilation were lacking and this study indicates that measurement of EGJ distensibility with the EndoFLIP technique prior to or directly after pneumatic balloon dilation has no additional role in the prediction of clinical treatment outcome.

Strengths of this study are the homogeneous population of only newly diagnosed achalasia patients, the large group of patients compared to previous studies, the standardized treatment protocol and assessment of clinical outcome at one-year follow-up. The small number of patients in different categories (i.e. achalasia subtypes and response rate) is a possible limitation. Moreover, EndoFLIP measurements were performed under moderate conscious sedation with midazolam in achalasia patients whereas healthy controls received no sedation. Previous studies found no effect of midazolam on LES resting pressure and effects of general anesthesia on EGJ distensibility are considered negligible. ${ }^{27,28}$ Therefore, we have insufficient evidence to 
assume that use of conscious sedation in achalasia patients has affected our data. In addition, we assume that the higher age in achalasia patients compared to healthy controls is not a limitation of the study as LES resting pressure deteriorates with advancing age; it is likely that the difference in EGJ distensibility would be even larger if we would compare achalasia patients with age-matched healthy controls. ${ }^{29}$

Interpretation of our data should be done with caution due to a number of limitations. First, consensus is lacking with regard to analysis of parameters obtained from EndoFLIP measurements of EGJ distensibility. Parameters used in our study were based on previous literature, and data currently available from the EndoFLIP output (i.e. EGJ distensibility was assessed based on the narrowest CSA and corresponding intra-bag pressure using a standardized volume-distension protocol). However, this does not exclude that alternative analytical techniques might have resulted in alternative or more accurate interpretations of our data. Second, a remarkable result in this study was the relatively high EGJ distensibility in newly diagnosed achalasia patients at the $30 \mathrm{ml}$ volumetric distension volume when compared to the 40 and $50 \mathrm{ml}$ distension volumes. Although the EndoFLIP technique has been validated down to $5 \mathrm{~mm}$ diameter, we cannot exclude that the narrow EGJ diameter in newly diagnosed achalasia patients prevents proper distension of the EndoFLIP balloon at low distension volumes. ${ }^{30}$ In addition, due to the narrow EGJ zone in achalasia, the peak pressure may not be exerted at the point where the pressure transducer is located. As a result, intra-bag pressure might be underestimated with consequently an inaccurate measurement of EGJ distensibility. Therefore, EndoFLIP results, particularly at lower distension volumes, in newly diagnosed achalasia patients may appear unreliable, and results at the $30 \mathrm{ml}$ volumetric distension volume in this study should be interpreted with caution. Additional validation experiments of EGJ distensibility in this lower volume range may be warranted in achalasia patients to address this issue. In the future, it would be interesting to acquire additional information about the slope of the EGJ distensibility curve using a stepwise or ramp distension protocol, thereby offering correction for mechanoelastic influences of a narrow EGJ. Due to the small number of volumetric distensions performed in this study, we were unable to construct a distensibility slope as previously described by Kwiatek et al. for assessment of esophageal body distensibility in patients with eosinophilic esophagitis. ${ }^{31}$ It has to be noted, however, that the clinical relevance of information obtained from such distensibility slope of the EGJ remains to be established.

In conclusion, assessment of the functional obstruction at the level of the esophagogastric junction (EGJ) in newly diagnosed achalasia patients with the EndoFLIP technique is feasible, and EGJ distensibility differs between achalasia subtypes. Treatment of newly diagnosed achalasia patients with pneumatic balloon dilation improves EGJ distensibility. However, EGJ distensibility prior to or directly after pneumatic balloon dilation was not different between patients with good and 
poor clinical treatment outcome at one-year follow-up. Therefore, assessment of EGJ distensibility with the EndoFLIP technique, as carried out in this study with a limited number of distension steps, provides no additional information that is useful for clinical evaluation and management of newly diagnosed achalasia patients. 


\section{References}

1. Richter JE. Oesophageal motility disorders. Lancet. 2001;358(9284):823-8.

2. Podas T, Eaden J, Mayberry M, Mayberry J. Achalasia: a critical review of epidemiological studies. Am J Gastroenterol. 1998;93(12):2345-7.

3. Gennaro N, Portale G, Gallo C, Rocchietto S, Caruso V, Costantini M, et al. Esophageal achalasia in the Veneto region: epidemiology and treatment. Epidemiology and treatment of achalasia. J Gastrointest Surg. 2011;15(3):423-8.

4. Kwiatek MA, Pandolfino JE, Hirano I, Kahrilas PJ. Esophagogastric junction distensibility assessed with an endoscopic functional luminal imaging probe (EndoFLIP). Gastrointest Endosc. 2010;72(2):272-8.

5. McMahon BP, Frokjaer JB, Liao D, Kunwald P, Drewes AM, Gregersen H. A new technique for evaluating sphincter function in visceral organs: application of the functional lumen imaging probe (FLIP) for the evaluation of the oesophago-gastric junction. Physiol Meas. 2005;26(5):823-36.

6. McMahon BP, Frokjaer JB, Kunwald P, Liao D, Funch-Jensen P, Drewes AM, et al. The functional lumen imaging probe (FLIP) for evaluation of the esophagogastric junction. Am J Physiol Gastrointest Liver Physiol. 2007;292(1):G377-84.

7. Gregersen H, Djurhuus JC. Impedance planimetry: a new approach to biomechanical intestinal wall properties. Dig Dis. 1991;9(6):332-40.

8. Pandolfino JE, de Ruigh A, Nicodeme F, Xiao Y, Boris L, Kahrilas PJ. Distensibility of the esophagogastric junction assessed with the functional lumen imaging probe (FLIP) in achalasia patients. Neurogastroenterol Motil. 2013;25(6):496-501.

9. Rohof WO, Hirsch DP, Kessing BF, Boeckxstaens GE. Efficacy of treatment for patients with achalasia depends on the distensibility of the esophagogastric junction. Gastroenterology. 2012;143(2):328-35.

10. Kahrilas PJ, Boeckxstaens G. The spectrum of achalasia: lessons from studies of pathophysiology and high-resolution manometry. Gastroenterology. 2013;145(5):954-65.

11. Roman S, Zerbib F, Queneherve L, Clermidy H, Varannes SB, Mion F. The Chicago classification for achalasia in a French multicentric cohort. Dig Liver Dis. 2012;44(12):976-80.

12. Pandolfino JE, Kwiatek MA, Nealis T, Bulsiewicz W, Post J, Kahrilas PJ. Achalasia: a new clinically relevant classification by high-resolution manometry. Gastroenterology. 2008;135(5):1526-33.

13. Richter JE. Achalasia - an update. J Neurogastroenterol Motil. 2010;16(3):232-42.

14. Richter JE, Boeckxstaens GE. Management of achalasia: surgery or pneumatic dilation. Gut. 2011;60(6):869-76.

15. Vaezi MF, Pandolfino JE, Vela MF. ACG clinical guideline: diagnosis and management of achalasia. Am J Gastroenterol. 2013;108(8):1238-49; quiz 50.

16. Verlaan T, Rohof WO, Bredenoord AJ, Eberl S, Rosch T, Fockens P. Effect of peroral endoscopic myotomy on esophagogastric junction physiology in patients with achalasia. Gastrointest Endosc. 2013;78(1):39-44.

17. Teitelbaum EN, Soper NJ, Pandolfino JE, Kahrilas PJ, Boris L, Nicodeme F, et al. An extended proximal esophageal myotomy is necessary to normalize EGJ distensibility during Heller myotomy for achalasia, but not POEM. Surg Endosc. 2014;28(10):2840-7.

18. Teitelbaum EN, Soper NJ, Pandolfino JE, Kahrilas PJ, Hirano I, Boris L, et al. Esophagogastric junction distensibility measurements during Heller myotomy and POEM for achalasia predict postoperative symptomatic outcomes. Surg Endosc. 2014.

19. Ghosh SK, Pandolfino JE, Rice J, Clarke JO, Kwiatek M, Kahrilas PJ. Impaired deglutitive EGJ relaxation in clinical esophageal manometry: a quantitative analysis of 400 patients and 75 controls. Am J Physiol Gastrointest Liver Physiol. 2007;293(4):G878-85.

20. Boeckxstaens GE, Annese V, des Varannes SB, Chaussade S, Costantini M, Cuttitta A, et al. Pneumatic dilation versus laparoscopic Heller's myotomy for idiopathic achalasia. N Engl J Med. 2011;364(19):1807-16.

21. Rohof WO, Salvador R, Annese V, Bruley des Varannes S, Chaussade S, Costantini M, et al. Outcomes of treatment for achalasia depend on manometric subtype. Gastroenterology. 2013;144(4):718-25; quiz e13-4. 
22. Lee JY, Kim N, Kim SE, Choi YJ, Kang KK, Oh DH, et al. Clinical characteristics and treatment outcomes of 3 subtypes of achalasia according to the chicago classification in a tertiary institute in Korea. J Neurogastroenterol Motil. 2013;19(4):485-94.

23. Pratap N, Kalapala R, Darisetty S, Joshi N, Ramchandani M, Banerjee R, et al. Achalasia cardia subtyping by high-resolution manometry predicts the therapeutic outcome of pneumatic balloon dilatation. J Neurogastroenterol. 2011;17(1):48-53.

24. Schiano TD, Fisher RS, Parkman HP, Cohen S, Dabezies M, Miller LS. Use of high-resolution endoscopic ultrasonography to assess esophageal wall damage after pneumatic dilation and botulinum toxin injection to treat achalasia. Gastrointestinal Endoscopy. 1996;44(2):151-7.

25. Hong SJ, Bhargava V, Jiang YF, Denboer D, Mittal RK. A Unique esophageal motor pattern that involves longitudinal muscles Is responsible for emptying in achalasia esophagus. Gastroenterology. 2010;139(1):102-11.

26. Tucker E, Sweis R, Anggiansah A, Wong T, Telakis E, Knowles K, et al. Measurement of esophagogastric junction cross-sectional area and distensibility by an endolumenal functional lumen imaging probe for the diagnosis of gastroesophageal reflux disease. Neurogastroent Motil. 2013;25(11): 904-10.

27. Marsh JK, Hoffman SM, Dmuchowski CF. Effect of intravenous midazolam on esophageal motility testing in normal human volunteers. Am J Gastroenterol. 1993;88(6):860-3.

28. Nathanson LK, Brunott N, Cavallucci D. Adult esophagogastric junction distensibility during general anesthesia assessed with an endoscopic functional luminal imaging probe (EndoFLIP(R)). Surg Endosc. 2012;26(4):1051-5.

29. Grande L, Lacima G, Ros E, Pera M, Ascaso C, Visa J, et al. Deterioration of esophageal motility with age: a manometric study of 79 healthy subjects. Am J Gastroenterol. 1999;94(7):1795-801.

30. Keating FOJ. Accuracy of the EndoFLIP ${ }^{\mathrm{tm}}$ Functional Lumen Imaging Probe. Surg Endosc. 2009;23:S287.

31. Kwiatek MA, Hirano I, Kahrilas PJ, Rothe J, Luger D, Pandolfino JE. Mechanical properties of the esophagus in eosinophilic esophagitis. Gastroenterology. 2011;140(1):82-90. 


\section{Chapter 5}

\section{Effect of Transoral Incisionless Fundoplication on reflux mechanisms}

N.F. Rinsma, F.G.M. Smeets, D.W. Bruls, B.F. Kessing, N.D. Bouvy, A.A.M. Masclee, J.M. Conchillo 


\section{Abstract}

\section{Objectives}

Transoral Incisionless Fundoplication (TIF) is a new endoscopic treatment option for gastroesophageal reflux disease (GERD). The mechanisms underlying the antireflux effect of this new procedure have not been studied. We therefore conducted this explorative study to evaluate the effect of TIF on reflux mechanisms, focussing on transient lower esophageal sphincter relaxations (TLESRs) and esophagogastric junction (EGJ) distensibility.

\section{Methods}

Fifteen GERD patients (11 males; mean age 41, range 23-66 yrs), dissatisfied with medical treatment were studied before and six months after TIF. A 90 minutes postprandial combined high-resolution manometry and impedance-pH monitoring and an ambulatory 24-h pH-impedance monitoring were performed. Esophagogastric junction distensibility was evaluated using an endoscopic functional luminal imaging probe before and direct after the procedure.

\section{Results}

TIF reduced the number of postprandial TLESRs $(16.8 \pm 1.5$ vs. $9.2 \pm 1.3, p<0.01)$ and the number of postprandial TLESRs associated with reflux ( $11.1 \pm 1.6$ vs. $5.6 \pm 0.6, p<0.01)$, but the proportion of TLESRs associated with reflux was unaltered $(67.6 \pm 6.9 \%$ vs. $69.9 \pm 6.3 \%$ ). TIF also led to a decrease in the number and proximal extent of reflux episodes and an improvement of acid exposure in the upright position; on the contrary TIF had no effect on the number of gas reflux episodes. EGJ distensibility was reduced after the procedure $\left(2.4 \pm 0.3\right.$ vs. $\left.1.6 \pm 0.2 \mathrm{~mm}^{2} / \mathrm{mmHg}, p<0.05\right)$.

\section{Conclusions}

Transoral incisionless fundoplication reduced the number of postprandial TLESRs, the number of TLESRs associated with reflux and EGJ distensibility. This resulted in a reduction of the number and proximal extent of reflux episodes and improvement of acid exposure in the upright position. The antireflux effect of TIF showed to be selective for liquid containing reflux only, thereby preserving the ability of venting gastric air. 


\section{Introduction}

In the past decade, new endoscopic approaches for the treatment of gastroesophageal reflux disease (GERD) have been introduced to fill the therapeutic gap between treatment with proton pump inhibitors (PPIs) and laparoscopic antireflux surgery (LARS). To date, most of these endoscopic techniques have failed, either because of a lack of long-term efficacy or due to complications of the procedure. ${ }^{1-4}$

Transoral incisionless fundoplication (TIF) using the EsophyX device is a recently developed endoscopic treatment option for GERD. In this procedure, a partial fundoplication is created under general anesthesia by sequential retractions of tissue, fixed by multiple transmurally placed polypropylene fasteners. ${ }^{5}$ Since its first introduction, several modifications have been made to the EsophyX device and to the TIF procedure. Using the TIF 1.0 technique, a neo-gastroesophageal valve was created by gastro-gastric plication, but modifications of fastener deployment led to a longer valve constructed by esophagogastric plication (TIF 2.0 technique) ${ }^{6,7}$ Uncontrolled clinical trials reported promising results, with respect to both symptom scores and PPI use, as well as in objective reflux parameters. ${ }^{8-11}$ The introduction of the TIF 2.0 technique and the extensive experience of the operating team have led to improved results in our medical center. A randomized controlled trial comparing TIF with PPI therapy was initiated, and long-term follow-up data from this trial are awaited; in the context of this trial, we performed the present study, to assess the effect of TIF 2.0 on reflux mechanisms.

Several studies investigating the effect of LARS on reflux mechanisms have shown a reduction in both the number of transient lower esophageal sphincter relaxations (TLESRs) as well as the proportion of TLESRs associated with reflux. ${ }^{12,13}$ TLESRs have been identified as the predominant mechanism for reflux to occur in both healthy subjects and GERD patients. ${ }^{14-17}$ A reduction in the number of TLESRs after LARS is considered to contribute to the anti-reflux effect of the procedure. The proportion of TLESRs associated with reflux is higher in GERD patients than in healthy subjects, while the total number of TLESRs is in the same range in both groups. ${ }^{18,19}$ This difference in TLESRs associated with reflux may be explained by an increased distensibility of the esophagogastric junction (EGJ): under the same distending pressure, the EGJ opens to a greater extent in GERD patients, allowing gastric contents to reflux into the esophagus more easily. ${ }^{20}$ This observation has recently been confirmed employing a new technique designed to measure intraluminal distensibility with the endoscopic functional luminal imaging probe (EndoFLIP). ${ }^{21}$ We aimed to investigate the mechanisms underlying the anti-reflux effect of TIF in this first explorative study, focusing on the effect of TIF on TLESRs, TLESRs associated with reflux, and EGJ distensibility. 


\section{Methods}

\section{Study population}

From 2008 to 2012, transoral incisionless fundoplication was performed in 60 GERD patients who were enrolled in a prospective trial evaluating the TIF procedure at the Maastricht University Medical Center. Throughout this period, 15 of these patients (11 males, mean age 41 years (range 23-66 yr), mean body mass index 26.2 21.1 $\mathrm{kg} / \mathrm{m}^{2}$ ) agreed to participate in the present study investigating the effect of TIF on reflux mechanisms.

All patients had chronic (>6 months) GERD symptoms, such as heartburn, regurgitation or retrosternal pain and were offered TIF as they were dissatisfied with PPI treatment or unwilling to undergo lifelong PPI therapy. GERD was well documented by upper $\mathrm{Gl}$ endoscopy and 24-h impedance-pH monitoring, showing esophagitis and/or pathologic acid exposure time ( $\mathrm{pH}$ below 4 during $>4.0 \%$ of time) with a high ( $\geq 95 \%)$ symptom association probability (SAP). ${ }^{22}$ Eight out of 15 (53\%) patients had documented reflux esophagitis on baseline endoscopy. Three patients had a grade $A$ esophagitis, 4 patients grade $B$ and 1 patient grade C (L.A. classification). Preoperative endoscopy showed a small $(\leq 2 \mathrm{~cm})$ hiatal hernia in 9 patients. Additionally, the gastroesophageal flap valve grade according to the Hill classification was assessed in all patients: grade 1 was found in 1 patient, grade 2 in 7 patients, grade 3 in 5 patients and grade 4 in 2 patients. ${ }^{23}$

Exclusion criteria were: patients $<18$ years of age, severe esophageal motility disorder on manometry, hiatal hernia $>2 \mathrm{~cm}$ in length, a history of previous antireflux surgery, reflux esophagitis grade D (L.A. classification), Barrett's epithelium, esophageal stricture or ulcer, current pregnancy or severe comorbidities (including cardiopulmonary disease, portal hypertension, coagulation disorders, immunosuppression or morbid obesity). The protocol was approved by the medical ethical committee of the Maastricht University Medical Center (approved on June $9^{\text {th }}$ 2008) and written informed consent was obtained from all patients.

\section{Study protocol}

Patients were studied before and 6 months after the TIF-procedure. A test day consisted of two parts as shown in the flow chart (Figure 5.1). During the first part of 90 minutes, postprandial measurements were performed using combined stationary high-resolution manometry (HRM) and impedance-pH monitoring. The second part consisted of a 24-h ambulatory impedance-pH monitoring. For at least 5 days before testing, the use of antisecretory drugs or drugs that influence gastroesophageal motility was ceased. After an overnight fast, both the manometric and impedance-pH catheters were introduced transnasally. End expiratory lower esophageal sphincter 
(LES) resting pressure was determined shortly before patients consumed a standardised high-caloric (960 kCal) liquid meal consisting of Nutridrink Juice Style (400 ml, containing $16 \mathrm{~g}$ protein, $134 \mathrm{~g}$ carbohydrates $(\mathrm{pH} \mathrm{2.9)})$ and Nutricia Calogen ( $80 \mathrm{ml}$, containing $80 \mathrm{~g}$ fat ( $\mathrm{pH} \mathrm{5.6))} \mathrm{(Nutricia,} \mathrm{Zoetermeer,} \mathrm{The} \mathrm{Netherlands)} \mathrm{within} 15$ minutes. Subsequently, impedance-pH and HRM recordings were started simultaneously and were continued for 90 minutes. During this period patients were in an upright position and were not allowed to doze. The 24-h impedance-pH monitoring was started after the HRM catheter was removed.

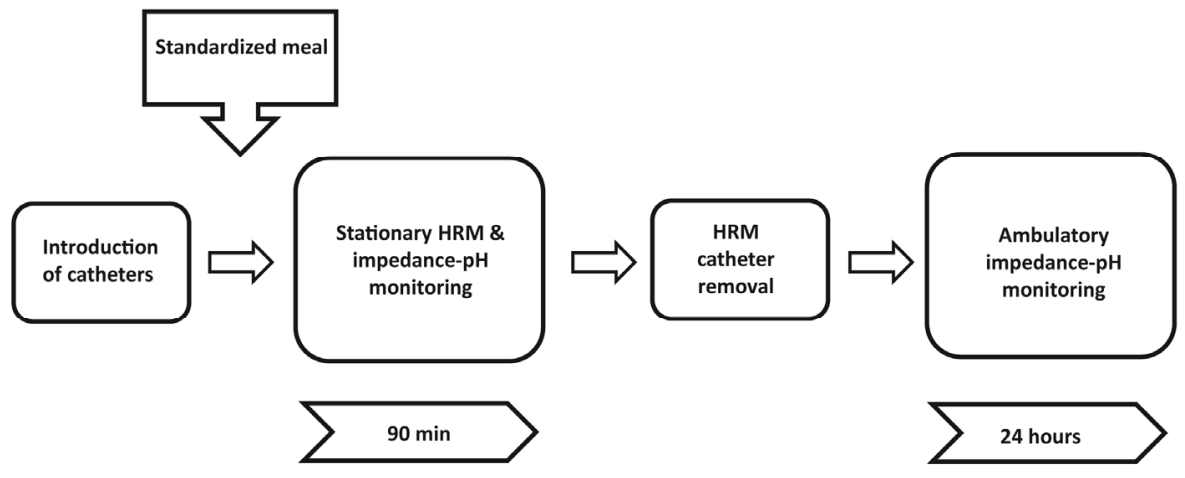

Figure 5.1 Flow chart of the study protocol. HRM, high-resolution manometry.

\section{Combined stationary high-resolution manometry and impedance-pH monitoring}

A solid-state HRM catheter (Unisensor AG, Attikon, Switzerland) was used, containing 36 pressure sensors spaced at a 1-cm interval and a maximum outer diameter of $5.3 \mathrm{~mm}$. Catheter position was considered correct when both the upper esophageal sphincter (UES) and LES were visible and at least 2 sensors were located in the stomach. Colour contour plots were displayed directly and saved at the end of the study. Alongside the HRM catheter, a combined impedance-pH catheter (Unisensor AG, Attikon, Switzerland) was positioned. The catheter contained six impedance segments and one ISFET $\mathrm{pH}$ electrode. The recording segments were located at 2-4, $4-6,6-8,8-10,14-16$ and $16-18 \mathrm{~cm}$ above the upper border of the LES. The $\mathrm{pH}$-electrode was positioned $5 \mathrm{~cm}$ above the upper border of the LES, according to the manometric colour plot. Impedance and $\mathrm{pH}$ signals were stored in a digital data logger (Ohmega, Medical Measurement Systems, Enschede, The Netherlands). After 90 minutes of recording, the HRM catheter was removed. 


\section{Ambulatory 24-h impedance-pH monitoring}

The impedance-pH catheter, left in situ, was used for the 24-h ambulatory monitoring. Patients were instructed to continue their regular diet, to report GERD symptoms and to keep a diary of their consumptions and body position (supine or upright) during the ambulatory measurement.

\section{Transoral Incisionless Fundoplication (TIF)}

Transoral incisionless fundoplication using the EsophyX-2 device (EndoGastric solutions, Inc., Redmond, WA, USA) was previously described in detail. ${ }^{24}$ In short, the procedure was performed under general endotracheal anesthesia by a team of a surgeon and a gastroenterologist. After transoral introduction of the device, the endoscope was positioned in retroflexion for optimal visualisation. A built-in vacuum invaginator was used to reposition the squamocolumnar junction below the diaphragm in case of a hiatal hernia. Subsequently, a tissue mold within the device was retroflexed and a helical retractor was inserted into the Z-line (Figure 5.2). With caudal retraction on the retractor, the tissue mold was used to rotate the fundus up and around the esophagus and circumferential deployment of multiple fasteners ensured a fixed neo-gastroesophageal valve. The procedures were carried out by a single surgeon (N.B.) and gastroenterologist (J.M.C.) at the Maastricht University Medical Center.

\section{Assessment of esophagogastric junction distensibility (EGJ) using EndoFLIP}

An endoscopic functional luminal imaging probe, EndoFLIP (Crospon Ltd, Galway, Ireland), was used to assess EGJ distensibility, according to previously described concepts. $^{21,25}$ In short, the EndoFLIP measurement system uses impedance planimetry to assess multiple cross-sectional areas (CSA) within a cylindrical bag placed over the EGJ. With simultaneous pressure measurements within the cylindrical bag, the distensibility (CSA/pressure response) over the EGJ can be assessed. During the procedure, shortly before introduction of the EsophyX device, the EndoFLIP probe was placed at the level of the esophagogastric junction according to previous endoscopic inspection. The bag was filled to $30 \mathrm{ml}$, as this volume appears to be the most relevant distension level. ${ }^{26,27}$ Corresponding cross-sectional area and distension pressure measurements were performed. EndoFLIP measurements were monitored in realtime to ensure the correct location of the bag. In case of migration of the bag, the measurements were repeated after repositioning of the bag. Directly after removal of the EsophyX device and the final endoscopic inspection, the EndoFLIP measurements were repeated while patients were still under general anaesthesia. 


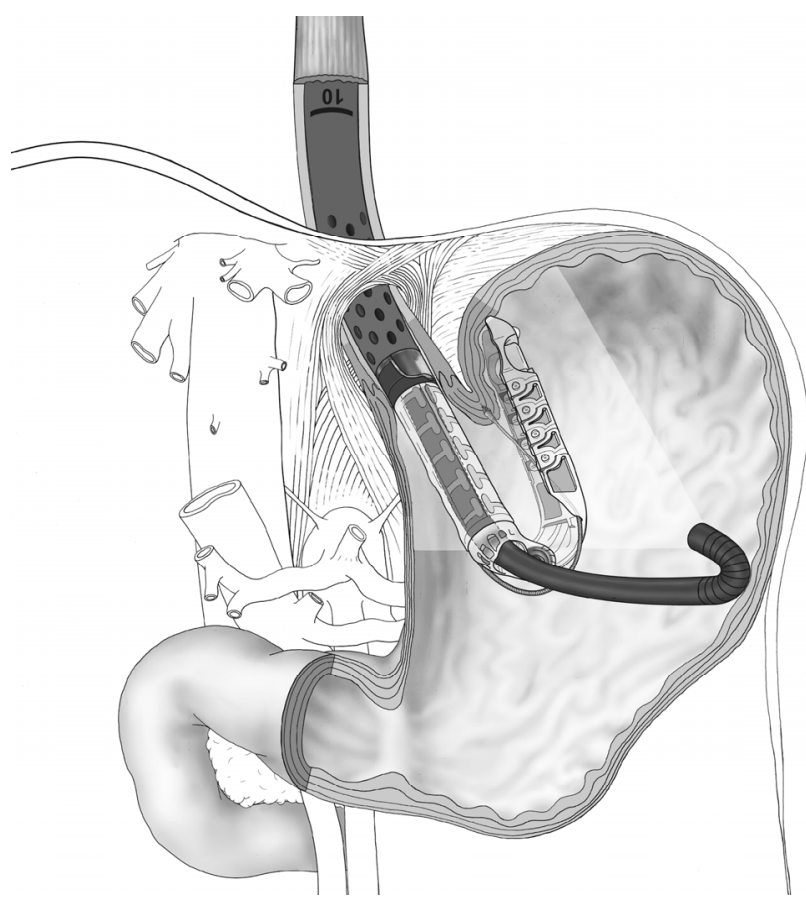

Figure 5.2 Illustration of transoral incisionless fundoplication with a depiction of the EsophyX device including the helical retractor inserted into the Z-line.

\section{Assessment of symptoms and medication use}

Pre- and postoperative symptoms were assessed using a disease-specific quality of life questionnaire (GERD-HRQL). ${ }^{28,29}$ The GERD-HRQL questionnaire assesses reflux related symptoms using a scale of 0 (no symptoms) to 5 (incapacitating). The maximum score for the GERD-HRQL is 50 points. Preoperative use of acid suppressive drugs was assessed at the outpatient clinic and again at six months follow-up.

\section{Data analysis}

Analysis of the postprandial high-resolution manometry measurements was done by a single investigator, who was blinded for the pre- or postoperative status of the patient. End expiratory LES pressure was determined before consumption of the liquid meal and referenced to end expiratory intragastric pressure. TLESRs were visually detected and scored with the colour contour plot, using the criteria developed by Holloway et al. and later modified for high-resolution manometry. ${ }^{30,31}$ Postprandial impedance-pH tracings were manually analyzed independent of the manometric findings by the same investigator. The analysis of the impedance-pH monitoring 
included the number of reflux episodes, composition (liquid, gas and mixed), proximal extent and acidity of the refluxate. According to previously described definitions, ${ }^{32}$ liquid reflux was defined as a fall $>50 \%$ in impedance compared to the baseline impedance level, beginning at the most distal recording site and reaching at least the next 2 proximal measuring segments. Gas reflux was defined as a sharp ( $>3000$ Ohms) rise in impedance that moved in a retrograde direction beginning at the most distal recording site and reaching at least the next 2 proximal measuring segments. Mixed reflux was defined as gas reflux occurring during or immediately preceding liquid reflux. Mixed and pure liquid reflux episodes were grouped as liquid containing reflux episodes for further analysis of the underlying mechanisms. Using the $\mathrm{pH}$-tracings, reflux episodes were classified as acid reflux when the nadir $\mathrm{pH}<4$, or non-acidic reflux when $\mathrm{pH} \geq 4$. Reflux episodes were classified as proximal (reaching $\geq 17 \mathrm{~cm}$ above the LES), mid-esophageal (7-17 cm above the LES) or distal $(\leq 7 \mathrm{~cm})$.

Data from postprandial HRM and impedance-pH monitoring were combined, analyzing the occurrence of liquid containing reflux and simultaneous TLESR or other mechanisms underlying the reflux episode. Mechanisms of liquid containing reflux were classified as TLESR-induced, swallow-induced, abdominal straining induced, or due to absent/low basal LES pressure. ${ }^{17,33}$ In case of liquid containing reflux episodes during movement-induced artefacts, the mechanism was defined as noninterpretable. The ambulatory 24-h impedance-pH measurements were manually analyzed in the same manner as the postprandial measurements, excluding the periods of meal consumption.

Analysis of the EndoFLIP measurements to assess the esophagogastric junction distensibility was based on the narrowest cross-sectional area (CSA) and the corresponding intrabag pressure. CSA and intrabag pressure were assessed by quantifying the $50^{\text {th }}$ percentile of each recording of 30 seconds. EGJ distensibility was calculated as the narrowest CSA in $\mathrm{mm}^{2}$ /intrabag pressure in $\mathrm{mmHg}$.

\section{Statistical analysis}

All data are shown as mean \pm SEM, unless stated otherwise. Data was checked for normality using the Kolmogorov-Smirnov test. Comparisons between pre-operative and postoperative measurements were performed using the Wilcoxon signed rank test for non-parametric data and paired T-test for parametric data. The significance level was set at $p<0.05$. All statistical analyses were performed using commercially available computer software (PASW Statistics 18 for Windows, (C) SPSS, Inc, 2009, Chicago, IL, USA). 


\section{Results}

Stationary esophageal manometry and impedance-pH monitoring (90 $\min )$

TIF resulted in a marked reduction of both the number of TLESRs (from $16.8 \pm 1.5$ to 9.2 $\pm 1.3 ; p<0.01)$ and the number of TLESRs associated with liquid-containing reflux after the procedure (from $11.1 \pm 1.6$ to $5.6 \pm 0.6$; $p<0.01$ ) (Figure 5.3). Although the number of TLESRs with liquid-containing reflux episodes decreased, the proportion of TLESRs associated with liquid-containing reflux was not influenced by the procedure (from $67.6 \pm 6.9$ to $69.9 \pm 6.3 \% ; p=0.80$ ) (Table 5.1).

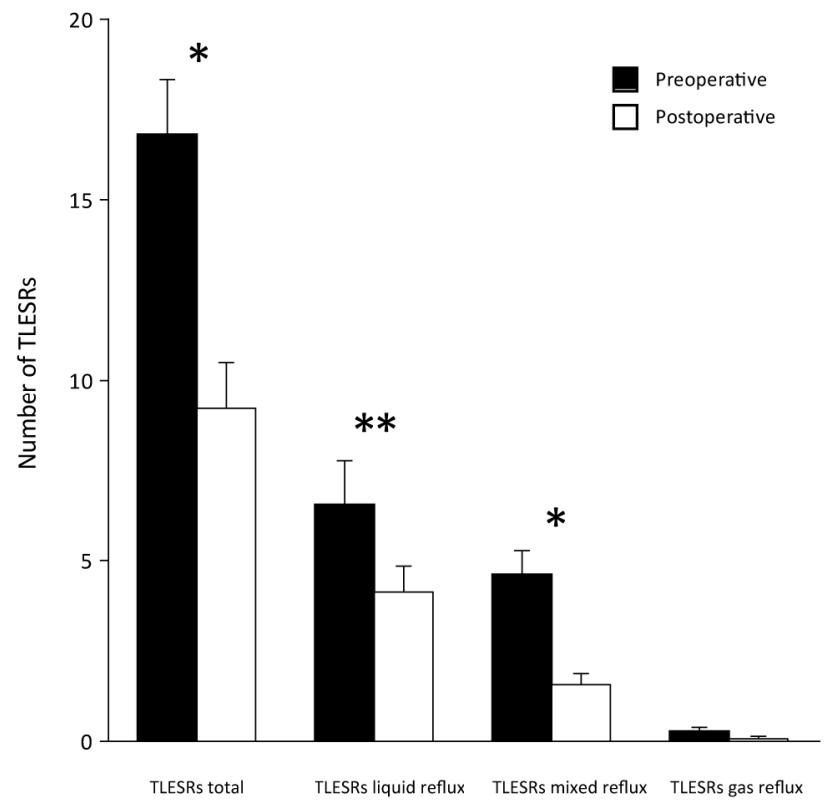

Figure 5.3 Number of transient lower esophageal sphincter relaxations (TLESRs) before and after transoral incisionless fundoplication during the $90 \mathrm{~min}$ postprandial period. $* \mathrm{p}<0.01, * *$ $p<0.05$.

Postprandial liquid containing reflux episodes were reduced after the procedure (from $18.8 \pm 2.1$ to $9.5 \pm 1.6 ; \mathrm{p}<0.01$ ), but TIF had no impact on the number of postprandial gas reflux episodes (Table 5.1). When determining the mechanism behind each liquid-containing reflux episode, TLESR was shown to be the dominant mechanism for reflux to occur, both before (63.5\%) and after TIF (68.1\%) (Table 5.2). The number of liquid-containing reflux episodes induced by TLESRs decreased after 
the procedure (from $10.1 \pm 1.6$ to $5.4 \pm 0.7 ; p<0.01$ ), but the proportion of liquidcontaining reflux episodes induced by a TLESR was unaltered. The number of liquidcontaining reflux episodes induced by a swallow or by other mechanisms was not significantly altered by the procedure (Table 5.2). Basal LES pressure in the fasted state was increased after the procedure (from $13.9 \pm 1.0$ to $20.5 \pm 1.8 \mathrm{mmHg} ; p<0.01$ ).

Table 5.1 Results of the combined high-resolution manometry (HRM) and impedance-pH measurements during the 90 min postprandial period before and after transoral incisionless fundoplication.

\begin{tabular}{lccc}
\hline & Preoperative & Postoperative & P-value \\
\hline Total number of TLESRs & $16.8 \pm 1.5$ & $9.2 \pm 1.3$ & $<0.01$ \\
TLESRs with liquid reflux & $6.5 \pm 1.2$ & $4.1 \pm 0.7$ & $<0.05$ \\
TLESRs with mixed reflux & $4.6 \pm 0.7$ & $1.6 \pm 0.3$ & $<0.01$ \\
TLESRs with gas reflux & $0.3 \pm 0.1$ & $0.1 \pm 0.1$ & $\mathrm{NS}$ \\
TLESRs with reflux (\%) & $67.6 \pm 6.9$ & $69.9 \pm 6.3$ & $\mathrm{NS}$ \\
& & & \\
Total reflux episodes & $18.8 \pm 2.1$ & $9.5 \pm 1.6$ & $<0.01$ \\
Liquid reflux episodes & $9.7 \pm 1.5$ & $6.0 \pm 1.2$ & $<0.05$ \\
Mixed reflux episodes & $7.6 \pm 0.8$ & $2.5 \pm 0.5$ & $<0.01$ \\
Gas reflux episodes & $1.5 \pm 0.4$ & $1.0 \pm 0.4$ & $\mathrm{NS}$ \\
\hline
\end{tabular}

NS: not significant. TLESR, transient lower esophageal sphincter relaxation

Table 5.2 Mechanisms underlying liquid containing reflux episodes of 15 GERD patients during 90 min postprandial period before and after transoral incisionless fundoplication.

\begin{tabular}{lccc}
\hline & Preoperative & Postoperative & p-value \\
\hline TLESR induced & $165(63.5 \%)$ & $81(68.1 \%)$ & $<0.01$ \\
Swallow-induced & $65(25.0 \%)$ & $29(24.4 \%)$ & N.S. \\
Abdominal straining & $11(4.2 \%)$ & $2(1.7 \%)$ & $<0.05$ \\
Low LES pressure & $13(5.0 \%)$ & $5(4.2 \%)$ & N.S. \\
Non-interpretable & $6(2.3 \%)$ & $2(1.7 \%)$ & N.S. \\
Total reflux episodes & & & $<0.001$ \\
\hline
\end{tabular}

NS: not significant. TLESR, transient lower esophageal sphincter relaxation; LES, lower esophageal sphincter.

\section{Ambulatory 24-h impedance-pH study}

Mean esophageal acid exposure time was not significantly reduced (from $10.9 \pm 1.9 \%$ to $7.3 \pm 1.6 \%, p=0.07) 6$ months after transoral incisionless fundoplication. However, in the upright position a significant reduction was observed (Table 5.3). TIF resulted in normalisation of the esophageal acid exposure time ( $\mathrm{pH}<4$ during $<4.0 \%$ of time) in $47 \%(7 / 15)$ of the patients. The esophageal acid exposure time of an additional $27 \%$ (4/15) of the patients improved after the procedure. No differences in reflux mechanisms or EGJ-characteristics were observed between patients with and without improved acid exposure time. 
Table 5.3 Results of the 24-h ambulatory impedance-pH measurements before and after transoral incisionless fundoplication.

\begin{tabular}{lccc}
\hline & Preoperative & Postoperative & p-value \\
\hline Total reflux episodes & $124.0 \pm 11.4$ & $104.4 \pm 13.7$ & 0.06 \\
Liquid reflux episodes & $30.4 \pm 3.5$ & $16.7 \pm 3.5$ & $<0.01$ \\
Mixed reflux episodes & $57.7 \pm 5.7$ & $45.1 \pm 6.1$ & $\mathrm{NS}$ \\
Gas reflux episodes & $35.9 \pm .7 .6$ & $42.6 \pm 10.2$ & $\mathrm{NS}$ \\
Proximal reflux episodes & $36.7 \pm 6.8$ & $15.3 \pm 2.9$ & $<0.05$ \\
Proximal reflux episodes (\%) & $39.6 \pm 4.9$ & $24.6 \pm 3.6$ & $<0.05$ \\
& & & \\
Total acid exposure time (pH<4) (\%) & $10.9 \pm 1.9$ & $7.3 \pm 1.6$ & 0.07 \\
Upright acid exposure time (\%) & $11.7 \pm 2.3$ & $6.6 \pm 1.0$ & $<0.05$ \\
Supine acid exposure time (\%) & $10.2 \pm 2.5$ & $8.4 \pm 3.0$ & $\mathrm{NS}$ \\
\hline
\end{tabular}

NS: not significant.

Analysis of 24-h impedance-pH tracings revealed a reduction in the number of liquid containing reflux episodes after fundoplication (from 88.1 \pm 6.1 to $61.8 \pm 7.0$, $\mathrm{p}<0.05$ ). The number of gas reflux episodes was not affected by the procedure (Table 5.3). The number and the percentage of proximal reflux episodes were significantly decreased $(p<0.05)$. Furthermore, the percentage of acid reflux episodes with duration longer than 5 minutes decreased significantly (from $5.0 \pm 1.2$ to $2.3 \pm 0.6$, $\mathrm{p}<0.05)$.

\section{Esophagogastric junction distensibility}

Correct placement of the EndoFLIP probe was achieved in all patients, as indicated by the hourglass shape of the probe during insufflation. Mean distending pressure within the EndoFLIP bag during $30 \mathrm{ml}$ distension was significantly higher after TIF (from $18.5 \pm 1.6$ to $21.8 \pm 1.8 \mathrm{mmHg}, \mathrm{p}<0.05$ ) whereas hiatal cross sectional area (CSA) was not reduced (from $40.6 \pm 5.4$ to $33.6 \pm 4.1 \mathrm{~mm}^{2}, p=0.27$ ). The mean EGJ distensibility (CSA/intrabag pressure) was significantly reduced after TIF (from $2.4 \pm 0.3$ to $1.6 \pm 0.2$ $\left.\mathrm{mm}^{2} / \mathrm{mmHg}, \mathrm{p}<0.05\right)$.

\section{Clinical assessment}

Transoral incisionless fundoplication reduced the GERD-HRQL score for heartburn (from $27.5 \pm 1.8$ to $13.2 \pm 2.4, \mathrm{p}<0.01$ ) (Figure 5.4). Symptomatic remission was achieved in 12 patients $(80 \%)$ after TIF, of which 8 patients also showed a reduction in acid exposure time. Three patients (20\%) reported persistent daily GERD symptoms after the procedure although acid exposure time was reduced below the normal range in 2 of these patients $(\mathrm{pH}<4<4.0 \%)$ and by $>50 \%$ in the third patient. All three patients still showed a positive symptom association probability on $\mathrm{pH}$-impedance monitoring after the procedure. No differences were observed in the number of TLESRs, the number of 
reflux episodes or EGJ-characteristics that could account for the persistent symptoms after TIF.

None of the patients reported new symptoms of gas-bloating or dysphagia after the procedure.

All patients were on a daily dosage of PPI's before the procedure. Ten out of $15(67 \%)$ patients reported complete cessation of antisecretory medication at six months after the procedure. One patient used antacids daily for mild symptoms and four patients $(27 \%)$ continued PPI use because of persistent symptoms after the procedure. However, 3 of those patients were on a lower dosage than before the procedure.

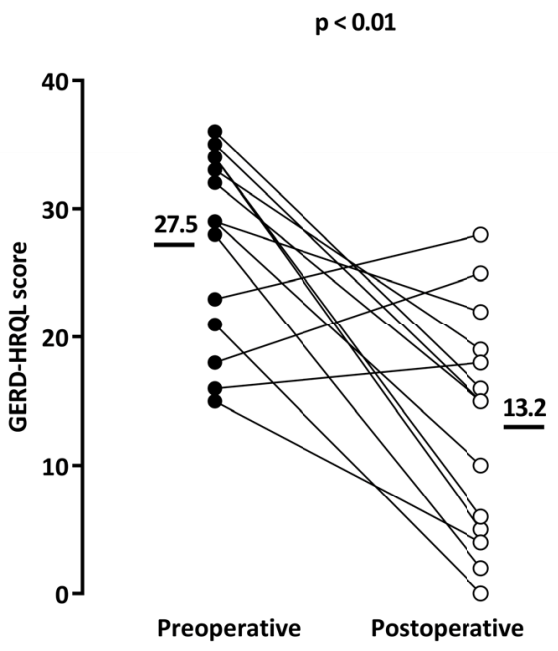

Figure 5.4 GERD-HRQL scores for each individual patient before (closed circles) and 6 months (open circles) after transoral incisionless fundoplication. Horizontal bars indicate mean values.

\section{Discussion}

In the context of an ongoing randomized trial by our research group we performed this first explorative study evaluating in detail the effect of transoral incisionless fundoplication (TIF) on reflux mechanisms and EGJ function. The major findings are that TIF reduced the number of postprandial TLESRs and the number and proximal extent of reflux episodes, which led to an improvement of acid exposure in the upright position after 6 months. Furthermore, EGJ distensibility was decreased as measured directly after the endoscopic procedure. 
Reduction of the number of TLESRs was previously described in studies evaluating the mechanisms of conventional antireflux surgery, and this mechanism is thought to contribute highly to the antireflux effect of conventional antireflux surgery. ${ }^{12,13,34}$ In addition to the reduction in the number of TLESRs, we also observed a marked effect of TIF on the number and proximal extent of reflux episodes, both during the postprandial and the ambulatory measurement. This led to a significant improvement of acid exposure time in the upright position, but not in the supine position. This finding could be due to a different profile of reflux mechanisms in the two positions; whereas in the upright position TLESRs are considered to be the dominant mechanism for reflux to occur, in the supine position, especially during sleep or in the presence of hiatal hernia, other mechanisms could be of more importance. ${ }^{15,35,36}$ TIF had a significant effect on the number of TLESRs, but may have a less distinct effect on the mechanisms causing reflux in supine position.

The neurological pathways involved in the vago-vagal reflex that leads to a TLESR are well described, however the mechanism by which fundoplication reduces the frequency of TLESRs remains to be determined. ${ }^{16,37-39}$ Several studies focussing on either the compliance of the proximal stomach ${ }^{40-42}$ or on postoperative vagal nerve damage ${ }^{41,43,44}$ have not been able to provide us with explanatory mechanisms. However, as shown in a recent study, localised distension of the EGJ itself increased the rate of TLESRs and was proposed as a potential mechanism for triggering of TLESRs. ${ }^{45}$ The authors suggest a role for stretch- and tension receptors in the EGJ region that trigger TLESRs as response to distension. The reduction of EGJ distensibility by fundoplication gives rise to the hypothesis that fundoplication increases the threshold for triggering of TLESRs by reducing EGJ distensibility. ${ }^{46,47}$ In the present study we observed a decrease in the number of TLESRs and a reduced EGJ distensibility after TIF, supporting the previously proposed hypothesis that the effect of TIF on the number of TLESRs may be due to the reduction of EGJ distensibility by the newly created fundic wrap. Further studies are needed to fully elucidate the mechanisms of fundoplication, especially the effect of the procedure on EGJ distensibility and the triggering of TLESRs, so that in the future surgeons are able to customize fundoplication in order to achieve not only a reduction in reflux events but also to prevent postoperative dysphagia and gas-bloating.

The frequency of postprandial TLESRs in the present study is comparable to that of healthy subjects as described in early reports. ${ }^{31,48,49}$ However, the proportion of TLESRs associated with liquid containing reflux was higher in GERD patients, a finding in accordance with previous studies. ${ }^{18,19}$ In healthy subjects, the occurrence of reflux during TLESRs is limited due to a small opening of the EGJ, whereas in GERD patients reflux of gastric juice can frequently occur during TLESRs due to loss of EGJ function..$^{20,21}$ The goal of fundoplication is to restore normal EGJ function, restricting the occurrence of liquid containing reflux during TLESRs, but allowing the occurrence of gas reflux. TIF was not able to reduce the proportion of TLESRs associated with 
liquid containing reflux in the present study, in contrast to LARS. ${ }^{12,13}$ We hypothesize that the opening dimension of the EGJ during TLESRs remained large enough to allow the occurrence of liquid containing reflux. Although we were not able to measure EGJ opening dimensions during TLESRs, we indeed observed no significant decrease in the cross-sectional area of the EGJ as measured with the EndoFLIP device after TIF.

During both postprandial and ambulatory measurements we observed a marked effect of TIF on the number of liquid containing reflux episodes, but the number of gas reflux episodes were unaffected by TIF. TLESRs are the predominant mechanism for reflux to occur, but are also considered to be an essential mechanism for venting intragastric air by belching. ${ }^{16}$ Conventional antireflux surgery can give rise to new gasrelated symptoms by reducing TLESRs. ${ }^{50,51}$ TIF had no effect on gas reflux, as seen during both ambulatory and postprandial measurements. None of the patients participating in the present study developed any gas-related symptoms after the procedure and earlier studies with more participants reported no gas-bloating in patients after TIF. ${ }^{52,53}$

Furthermore, the present study showed that TIF reduces both the number and percentage of proximal reflux episodes and this finding was also reported in GERD patients after Nissen fundoplication. ${ }^{13}$ The proximal extent of the refluxate is thought to be related to the volume of the refluxate and to contribute to symptom perception. ${ }^{54-57}$ We speculate that the observed reduction in EGJ distensibility after TIF may result in a reduction of the volume of the refluxate, thereby reducing symptom perception.

The present study was not primarily designed or powered to assess the clinical efficacy of TIF, but we observed an improvement in symptom scores as measured with GERD-HRQL, in PPI use and in acid exposure time in the upright position. Long term data from an ongoing randomized trial by our research group comparing TIF with PPI therapy in GERD patients are awaited to fully assess the antireflux effect of the procedure.

In summary, we aimed to explore the mechanisms underlying the antireflux effect of transoral incisionless fundoplication. The procedure significantly reduced the number of TLESRs and the number of TLESRs associated with liquid containing reflux. This effect may be due to the observed reduction of EGJ distensibility, raising the threshold for triggering TLESRs. This resulted in a reduction of the number of liquid containing reflux episodes and improvement in esophageal acid exposure in the upright position. However, the antireflux effect of TIF showed to be selective for liquid containing reflux, thereby maintaining ability of venting gastric air. Further randomized controlled trials are required to assess the antireflux effect and long term efficacy of transoral incisionless fundoplication. 


\section{References}

1. Schiefke I, Zabel-Langhennig A, Neumann S, Feisthammel J, Moessner J, Caca K. Long term failure of endoscopic gastroplication (EndoCinch). Gut. 2005;54:752-758.

2. Schwartz MP, Wellink H, Gooszen HG, Conchillo JM, Samsom M, Smout AJ. Endoscopic gastroplication for the treatment of gastro-oesophageal reflux disease: a randomised, sham-controlled trial. Gut. 2007;56:20-28.

3. Rothstein R, Filipi C, Caca K, Pruitt R, Mergener K, Torquati A, Haber G, Chen Y, Chang K, Wong D, Deviere J, Pleskow D, Lightdale C, Ades A, Kozarek R, Richards W, Lembo A. Endoscopic full-thickness plication for the treatment of gastroesophageal reflux disease: A randomized, sham-controlled trial. Gastroenterology. 2006;131:704-712.

4. Arts J, Bisschops R, Blondeau K, Farre R, Vos R, Holvoet L, Caenepeel P, Lerut A, Tack J. A double-blind sham-controlled study of the effect of radiofrequency energy on symptoms and distensibility of the gastro-esophageal junction in GERD. Am J Gastroenterol. 2012;107:222-230.

5. Cadiere GB, Rajan A, Germay O, Himpens J. Endoluminal fundoplication by a transoral device for the treatment of GERD: A feasibility study. Surg Endosc. 2008;22:333-342.

6. Jobe BA, O'Rourke RW, McMahon BP, Gravesen F, Lorenzo C, Hunter JG, Bronner M, Kraemer SJ. Transoral endoscopic fundoplication in the treatment of gastroesophageal reflux disease: the anatomic and physiologic basis for reconstruction of the esophagogastric junction using a novel device. Ann Surg. 2008;248:69-76.

7. Bell RC, Cadiere GB. Transoral rotational esophagogastric fundoplication: technical, anatomical, and safety considerations. Surg Endosc. 2011;25:2387-2399.

8. Cadiere GB, Van Sante N, Graves JE, Gawlicka AK, Rajan A. Two-year results of a feasibility study on antireflux transoral incisionless fundoplication using EsophyX. Surg Endosc. 2009;23:957-964.

9. Bell RC, Freeman KD. Clinical and pH-metric outcomes of transoral esophagogastric fundoplication for the treatment of gastroesophageal reflux disease. Surg Endosc. 2011;25:1975-1984.

10. Testoni PA, Vailati C, Testoni S, Corsetti M. Transoral incisionless fundoplication (TIF 2.0) with EsophyX for gastroesophageal reflux disease: long-term results and findings affecting outcome. Surg Endosc. 2012;26:1425-1435.

11. Trad KS, Turgeon DG, Deljkich E. Long-term outcomes after transoral incisionless fundoplication in patients with GERD and LPR symptoms. Surg Endosc. 2012;26:650-660.

12. Lindeboom MA, Ringers J, Straathof JW, van Rijn PJ, Neijenhuis P, Masclee AA. Effect of laparoscopic partial fundoplication on reflux mechanisms. Am J Gastroenterol. 2003;98:29-34.

13. Bredenoord AJ, Draaisma WA, Weusten BL, Gooszen HG, Smout AJ. Mechanisms of acid, weakly acidic and gas reflux after anti-reflux surgery. Gut. 2008;57:161-166.

14. Mittal RK, McCallum RW. Characteristics and frequency of transient relaxations of the lower esophageal sphincter in patients with reflux esophagitis. Gastroenterology. 1988;95:593-599.

15. Schoeman MN, Tippett MD, Akkermans LM, Dent J, Holloway RH. Mechanisms of gastroesophageal reflux in ambulant healthy human subjects. Gastroenterology. 1995;108:83-91.

16. Mittal RK, Holloway RH, Penagini R, Blackshaw LA, Dent J. Transient lower esophageal sphincter relaxation. Gastroenterology. 1995; 109:601-610.

17. Penagini R, Schoeman MN, Dent J, Tippett MD, Holloway RH. Motor events underlying gastrooesophageal reflux in ambulant patients with reflux oesophagitis. Neurogastroenterol Motil. 1996;8:131-141.

18. Trudgill NJ, Riley SA. Transient lower esophageal sphincter relaxations are no more frequent in patients with gastroesophageal reflux disease than in asymptomatic volunteers. Am J Gastroenterol. 2001;96:2569-2574.

19. Iwakiri K, Hayashi Y, Kotoyori M, Tanaka Y, Kawakami A, Sakamoto C, Holloway RH. Transient lower esophageal sphincter relaxations (TLESRs) are the major mechanism of gastroesophageal reflux but are not the cause of reflux disease. Dig Dis Sci. 2005;50:1072-1077. 
20. Pandolfino JE, Shi G, Trueworthy B, Kahrilas PJ. Esophagogastric junction opening during relaxation distinguishes nonhernia reflux patients, hernia patients, and normal subjects. Gastroenterology. 2003;125:1018-1024.

21. Kwiatek MA, Pandolfino JE, Hirano I, Kahrilas PJ. Esophagogastric junction distensibility assessed with an endoscopic functional luminal imaging probe (EndoFLIP). Gastrointest Endosc. 2010;72:272-278.

22. Masclee AA, de Best AC, de Graaf R, Cluysenaer OJ, Jansen JB. Ambulatory 24-hour pH-metry in the diagnosis of gastroesophageal reflux disease. Determination of criteria and relation to endoscopy. Scand J Gastroenterol. 1990;25:225-230.

23. Hill LD, Kozarek RA, Kraemer SJ, Aye RW, Mercer CD, Low DE, Pope CE, 2nd. The gastroesophageal flap valve: in vitro and in vivo observations. Gastrointest Endosc. 1996;44:541-547.

24. Cadiere GB, Buset M, Muls V, Rajan A, Rosch T, Eckardt AJ, Weerts J, Bastens B, Costamagna G, Marchese M, Louis H, Mana F, Sermon F, Gawlicka AK, Daniel MA, Deviere J. Antireflux transoral incisionless fundoplication using EsophyX: 12-month results of a prospective multicenter study. World J Surg. 2008;32:1676-1688.

25. McMahon BP, Frokjaer JB, Kunwald P, Liao D, Funch-Jensen $P$, Drewes AM, Gregersen $H$. The functional lumen imaging probe (FLIP) for evaluation of the esophagogastric junction. Am J Physiol Gastrointest Liver Physiol. 2007;292:G377-384.

26. Smeets F, Bouvy, ND, Koek, GH, Masclee, AM, Conchillo, JM. Esophagogastric junction (EGJ) distensibility in GERD patients as measured with an endoscopic functional luminal imaging probe: correlation with endoscopic and pH-impedance reflux parameters. Gastroenterology. 2012;142: S-424.

27. Nathanson LK, Brunott N, Cavallucci D. Adult esophagogastric junction distensibility during general anesthesia assessed with an endoscopic functional luminal imaging probe (EndoFLIP(R)). Surg Endosc. 2012;26:1051-1055.

28. Velanovich V. The development of the GERD-HRQL symptom severity instrument. Dis Esophagus. 2007;20:130-134.

29. Velanovich V, Vallance SR, Gusz JR, Tapia FV, Harkabus MA. Quality of life scale for gastroesophageal reflux disease. J Am Coll Surg. 1996;183:217-224.

30. Holloway RH, Penagini R, Ireland AC. Criteria for objective definition of transient lower esophageal sphincter relaxation. Am J Physiol. 1995;268:G128-133.

31. Bredenoord AJ, Weusten BL, Timmer R, Smout AJ. Sleeve sensor versus high-resolution manometry for the detection of transient lower esophageal sphincter relaxations. Am J Physiol Gastrointest Liver Physiol. 2005;288:G1190-1194.

32. Sifrim D, Holloway R, Silny J, Xin Z, Tack J, Lerut A, Janssens J. Acid, nonacid, and gas reflux in patients with gastroesophageal reflux disease during ambulatory 24-hour $\mathrm{pH}$-impedance recordings. Gastroenterology. 2001;120:1588-1598.

33. Dent J, Holloway RH, Toouli J, Dodds WJ. Mechanisms of lower oesophageal sphincter incompetence in patients with symptomatic gastrooesophageal reflux. Gut. 1988;29:1020-1028.

34. Ireland AC, Holloway $\mathrm{RH}$, Toouli J, Dent J. Mechanisms underlying the antireflux action of fundoplication. Gut. 1993;34:303-308.

35. Freidin N, Mittal RK, McCallum RW. Does body posture affect the incidence and mechanism of gastrooesophageal reflux? Gut. 1991;32:133-136.

36. van Herwaarden MA, Samsom M, Smout AJ. Excess gastroesophageal reflux in patients with hiatus hernia is caused by mechanisms other than transient LES relaxations. Gastroenterology. 2000;119:1439-1446.

37. Holloway RH, Hongo M, Berger K, McCallum RW. Gastric distention: a mechanism for postprandial gastroesophageal reflux. Gastroenterology 1985;89:779-784.

38. Franzi SJ, Martin CJ, Cox MR, Dent J. Response of canine lower esophageal sphincter to gastric distension. Am J Physiol. 1990;259:G380-385

39. Kessing BF, Conchillo JM, Bredenoord AJ, Smout AJ, Masclee AA. Review article: the clinical relevance of transient lower oesophageal sphincter relaxations in gastro-oesophageal reflux disease. Aliment Pharmacol Ther. 2011;33:650-661. 
40. Wijnhoven BP, Salet GA, Roelofs JM, Smout AJ, Akkermans LM, Gooszen HG. Function of the proximal stomach after Nissen fundoplication. Br J Surg. 1998;5:267-271.

41. Vu MK, Straathof JW, v d Schaar PJ, Arndt JW, Ringers J, Lamers CB, Masclee AA. Motor and sensory function of the proximal stomach in reflux disease and after laparoscopic Nissen fundoplication. Am J Gastroenterol. 1999;94:1481-1489.

42. Scheffer RC, Tatum RP, Shi G, Akkermans LM, Joehl RJ, Kahrilas PJ. Reduced tLESR elicitation in response to gastric distension in fundoplication patients. American journal of physiology Gastrointest Liver Physiol. 2003;284:G815-820.

43. Vu MK, Ringers J, Arndt JW, Lamers CB, Masclee AA. Prospective study of the effect of laparoscopic hemifundoplication on motor and sensory function of the proximal stomach. Br J Surg. 2000;87:338343.

44. Lindeboom MY, Ringers J, van Rijn PJ, Neijenhuis P, Stokkel MP, Masclee AA. Gastric emptying and vagus nerve function after laparoscopic partial fundoplication. Ann Surg. 2004;240:785-790.

45. van Wijk MP, Blackshaw LA, Dent J, Benninga MA, Davidson GP, Omari TI. Distension of the esophagogastric junction augments triggering of transient lower esophageal sphincter relaxation. Am J Physiol Gastrointest Liver Physiol. 2011;301:G713-718.

46. Pandolfino JE, Curry J, Shi G, Joehl RJ, Brasseur JG, Kahrilas PJ. Restoration of normal distensive characteristics of the esophago-gastric junction after fundoplication. Ann Surg. 2005;242:43-48.

47. Kwiatek MA, Kahrilas K, Soper NJ, Bulsiewicz WJ, McMahon BP, Gregersen H, Pandolfino JE. Esophagogastric junction distensibility after fundoplication assessed with a novel functional luminal imaging probe. J Gastrointest Surg. 2010;14:268-276.

48. Bredenoord AJ, Weusten BL, Timmer R, Smout AJ. Gastro-oesophageal reflux of liquids and gas during transient lower oesophageal sphincter relaxations. Neurogastroenterol Motil. 2006;18:888-893.

49. Pandolfino JE, Zhang QG, Ghosh SK, Han A, Boniquit C, Kahrilas PJ. Transient lower esophageal sphincter relaxations and reflux: mechanistic analysis using concurrent fluoroscopy and highresolution manometry. Gastroenterology. 2006;131:1725-1733.

50. Straathof JW, Ringers J, Lamers CB, Masclee AA. Provocation of transient lower esophageal sphincter relaxations by gastric distension with air. Am J Gastroenterol. 2001;96:2317-2323.

51. Broeders JA, Mauritz FA, Ahmed Ali U, Draaisma WA, Ruurda JP, Gooszen HG, Smout AJ, Broeders IA, Hazebroek EJ. Systematic review and meta-analysis of laparoscopic Nissen (posterior total) versus Toupet (posterior partial) fundoplication for gastro-oesophageal reflux disease. $\mathrm{Br} \mathrm{J}$ Surg. 2010;97:1318-1330.

52. Bell RC, Freeman KD. Clinical and pH-metric outcomes of transoral esophagogastric fundoplication for the treatment of gastroesophageal reflux disease. Surg Endosc. 2011;25:1975-1984

53. Bell RC, Mavrelis PG, Barnes WE, Dargis D, Carter BJ, Hoddinott KM, Sewell RW, Trad KS, Gill BD, Ihde GM. A prospective multicenter registry of patients with chronic gastroesophageal reflux disease receiving transoral incisionless fundoplication. J Am Coll Surg. 2012;215:794-809.

54. Weusten BL, Akkermans LM, vanBerge-Henegouwen GP, Smout AJ. Symptom perception in gastroesophageal reflux disease is dependent on spatiotemporal reflux characteristics. Gastroenterology. 1995;108: 1739-1744.

55. Cicala M, Emerenziani S, Caviglia R, Guarino MP, Vavassori P, Ribolsi M, Carotti S, Petitti T, Pallone F. Intra-oesophageal distribution and perception of acid reflux in patients with non-erosive gastrooesophageal reflux disease. Aliment Pharmacol Ther. 2003;18:605-613.

56. Bredenoord AJ, Weusten BL, Curvers WL, Timmer R, Smout AJ. Determinants of perception of heartburn and regurgitation. Gut. 2006;55:313-318.

57. Bredenoord AJ. Mechanisms of reflux perception in gastroesophageal reflux disease: a review. Am J Gastroenterol. 2012;107:8-15. 


\section{Chapter 6}

Does measurement of esophagogastric junction distensibility by EndoFLIP predict therapyresponsiveness to endoluminal fundoplication in patients with gastroesophageal reflux disease?

F.G.M. Smeets, D. Keszthelyi, N.D. Bouvy, A.A.M. Masclee, J.M. Conchillo J Neurogastroenterol Motil. 2015;21(2):255-264 


\section{Abstract}

\section{Background/Aims}

In patients with gastroesophageal reflux disease (GERD), an increased esophagogastric junction (EGJ) distensibility has been described. Assessment of EGJ distensibility with the EndoFLIP technique might identify patients responsive to Transoral Incisionless Fundoplication (TIF), whereas postoperative measurement of EGJ distensibility might provide insight into the antireflux mechanism of TIF. Therefore, we investigated the value of the EndoFLIP technique in GERD patients treated by TIF.

\section{Methods}

Forty-two GERD patients underwent EGJ distensibility measurement before TIF using the EndoFLIP technique. In a subgroup of 25 patients, EndoFLIP measurement was repeated both postoperative and at six months follow-up. Treatment outcome was assessed according to esophageal acid exposure time (AET; objective outcome) and symptom scores (clinical outcome) six months after TIF.

\section{Results}

Multiple logistic regression analysis showed that preoperative EGJ distensibility (OR $0.16,95 \% \mathrm{Cl} 0.03-0.78, \mathrm{p}=0.023$ ) and preoperative AET (OR 0.62, 95\% Cl 0.42-0.90, $\mathrm{p}=0.013$ ) were independent predictors for objective treatment outcome but not for clinical outcome after TIF. The best cut-off value for objective outcome was $2.3 \mathrm{~mm}^{2} / \mathrm{mmHg}$ for preoperative EGJ distensibility and $11 \%$ for preoperative AET. EGJ distensibility decreased direct postoperative from 2.0 (1.2-3.3) to 1.4 (1.0-2.2) $\mathrm{mm}^{2} / \mathrm{mmHg} \quad(p=0.014)$, but increased to $2.2(1.5-3.0)$ at six months follow-up ( $p=0.925$, compared to preoperative).

\section{Conclusions}

Preoperative EGJ distensibility and preoperative AET were independent predictors for objective treatment outcome but not for clinical outcome after TIF. According to our data, the EndoFLIP technique has no added value either in the preoperative diagnostic work-up or in the post-procedure evaluation of endoluminal antireflux therapy. 


\section{Introduction}

Pharmacological therapy with proton pump inhibitors (PPIs) is effective in controlling reflux symptoms and therefore considered the mainstay for GERD treatment. However, in case of unresponsiveness to PPIs or incomplete symptom relief, antireflux surgery should be considered. ${ }^{1}$ Antireflux surgery controls GERD by restoring the competence of the gastroesophageal junction. It should be taken into account that antireflux surgery is associated with short and long-term adverse effects including dysphagia and gas bloat. ${ }^{2}$ In the last decade, several endoscopic treatment options have been developed as less invasive alternatives to antireflux surgery. ${ }^{3}$ Most of the devices like Gatekeeper ${ }^{\mathrm{tm}}$ and Enteryx showed acceptable to good short-term outcomes but are no longer available due to poor long-term outcomes and the occurrence of complications. ${ }^{4}$ Transoral Incisionless Fundoplication (TIF) using the EsophyX device is a novel technique developed for treatment of GERD patients and previous studies demonstrated that TIF is a safe treatment option and effective in improving quality of life. ${ }^{5}$ However, in uncontrolled trials normalization of acid exposure time is only present in 37-50\% of patients with a lack of correlation between objective and subjective treatment outcomes assessed with $\mathrm{pH}$-metry and validated questionnaires. ${ }^{6-8}$ Previous studies have attempted to identify patient characteristics and objective parameters like LES resting pressure and the number of fasteners that may help to predict treatment outcome of the TIF procedure. ${ }^{9,10}$ However, up to now, no objective parameter is available to select patients for this endoluminal procedure.

Increased esophagogastric junction (EGJ) distensibility is considered a pathophysiologic factor in GERD as EGJ compliance determines the opening diameter of the EGJ and consequently the volume of reflux content into the esophagus. ${ }^{11,12}$ Surgical treatment options attempt to restore anatomic components of the antireflux barrier and previous studies using the barostat method have described a reduction in EGJ distensibility after laparoscopic Nissen fundoplication. ${ }^{13,14}$ Recently, a new endoscopic functional luminal imaging probe (EndoFLIP) has been developed to assess EGJ distensibility using impedance planimetry during volumetric distensions. Advantages of this technique compared to barostat are its use without need for additional fluoroscopy, real time dynamic imaging of the EGJ and fast assessment of EGJ distensibility patterns. ${ }^{15}$ Stratification of GERD patients according to EGJ distensibility might help to identify patients responsive to TIF and we hypothesize that patients with low preoperative EGJ distensibility will have better EGJ competence and consequently a better response to TIF compared to patients with high EGJ distensibility. Moreover, the use of the EndoFLIP in the postoperative period might provide more insight into the antireflux mechanism of this endoluminal fundoplication procedure. ${ }^{15}$ Therefore, we investigated the value of the EndoFLIP technique in GERD patients treated by an endoluminal fundoplication procedure. 


\section{Materials and methods}

\section{Patients}

Between 2009 and 2012, Transoral Incisionless Fundoplication was performed in 60 chronic GERD patients in a randomized controlled trial comparing TIF with acid suppressive medication at the Maastricht University Medical Center. Outcomes of this randomized controlled trial from our group are awaited with long-term data after endoluminal fundoplication (including safety, complications and need for additional treatment) and will be reported separately. In the present study, a post-hoc analysis was performed in 42 patients to investigate the value of the EndoFLIP technique in endoluminal antireflux surgery.

Patients were referred for antireflux surgery either because they were refractory, unsatisfied or unwilling to take lifelong acid suppressive medication. Patients underwent upper gastrointestinal endoscopy, $24 \mathrm{hr}$ pH-impedance monitoring and esophageal manometry as standard preoperative work-up for antireflux surgery.

Patients with chronic GERD symptoms (>6 months), esophagitis at upper gastrointestinal endoscopy and/or abnormal acid exposure time during $24 \mathrm{hr} \mathrm{pH}$ impedance monitoring ( $\mathrm{pH}<4$ during $>4 \%$ of time) were included. All patients were aged 18 to 75 years with a body mass index (BMI) below $35 \mathrm{~kg} / \mathrm{m}^{2}$ and normal lower esophageal sphincter (LES) resting pressure ( $<30 \mathrm{mmHg}$ ). Exclusion criteria were age $<18$ years, current pregnancy, severe reflux esophagitis grade D according to the Los Angeles classification, other esophageal diseases (including esophageal motility disorders, biopsy proven Barrett's esophagus, esophageal stricture or esophageal ulcer), previous antireflux surgery or severe comorbidities (including cardiopulmonary disease, portal hypertension, coagulation disorders, immunosuppression or morbid obesity). Patients with hiatal hernia $>2 \mathrm{~cm}$ were excluded, since previous studies demonstrated that complete reduction of hernias was more often achieved in patients with small hiatal hernia $(1-2 \mathrm{~cm})$ compared to patients with large hiatal hernia $(>3 \mathrm{~cm}) .^{10}$

The protocol was approved by the medical ethical committee of the Maastricht University Medical Center (METC 07-2-051, NL17303.068.07) and was registered NCT00857597 (clinicaltrials.gov). Written informed consent was obtained from all patients.

\section{Study protocol}

Symptom scores, upper gastrointestinal endoscopy and $24 \mathrm{hr} \mathrm{pH}$-impedance monitoring were performed preoperatively to assess whether patients were eligible for the TIF procedure and these measurements were repeated six months after the endoluminal fundoplication. Esophageal manometry was performed preoperatively to 

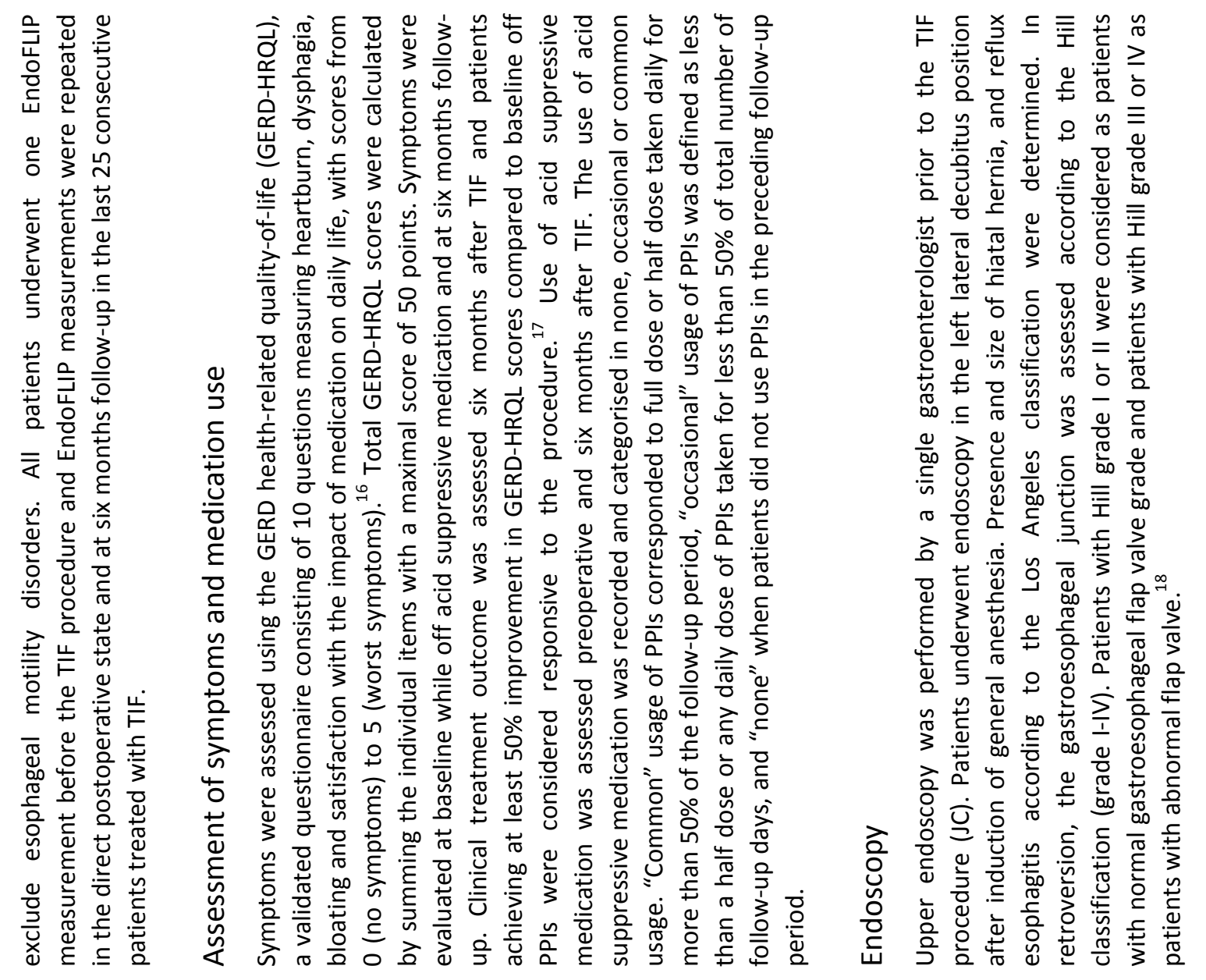

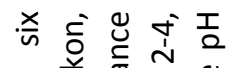

演美要

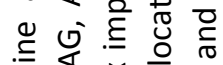

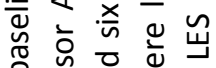

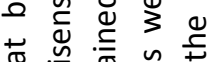

它政

है

这

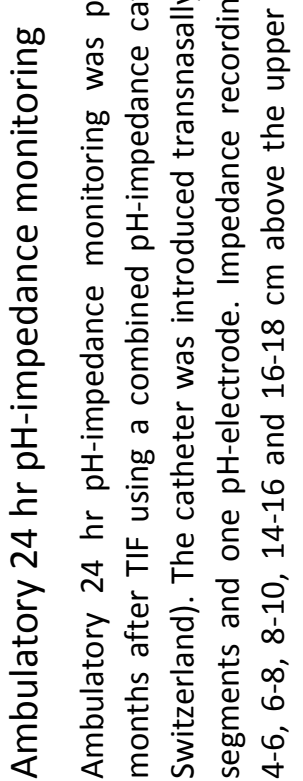


electrode was positioned $5 \mathrm{~cm}$ above the upper border of the LES, as determined during esophageal manometry. The $\mathrm{pH}$-impedance catheter was left in situ for 24 hours and data from the catheter were transmitted to and recorded on a portable recorder (Ohmega, Medical Measurement Systems, Enschede, The Netherlands). Patients received a personal diary to note GERD symptoms, meal periods and body position (upright or supine), meal periods were excluded from the analysis.

Analysis of $24 \mathrm{hr} \mathrm{pH}$-impedance measurements included acid exposure time (AET, $\%$ ) and number of liquid, proximal and acid reflux episodes. Reflux episodes with $\mathrm{pH}<4$ were classified as acidic reflux episodes and reflux episodes with $\mathrm{pH} \geq 4$ were classified as non-acidic reflux episodes. Reflux episodes reaching the two proximal recording segments were considered as proximal reflux episodes. Objective treatment outcome was assessed according to AET at six months follow-up. Patients with normalized AET ( $\mathrm{pH}<4$ for $\leq 4 \%$ of time) were considered as patients with a good objective treatment outcome whereas patients with persistent abnormal AET ( $\mathrm{pH}<4$ during $>4 \%$ of time) were considered as patients with a poor objective treatment outcome.

\section{Assessment of EGJ distensibility}

Esophagogastric junction distensibility was measured using a commercially available functional luminal imaging probe (EndoFLIP, Crospon Medical Devices, Galway, Ireland). ${ }^{15,19}$ The EndoFLIP probe consists of a catheter with a noncompliant bag on its distal end, which encloses 17 electrodes at $5 \mathrm{~mm}$ intervals. Cross sectional areas (CSAs) are determined for 16 balloon cross sections during volumetric distensions using impedance planimetry. One pressure sensor is located on the probe to determine intra-bag pressure allowing assessment of EGJ distensibility of the distended area. Baseline pressure was zeroed prior to insertion of the catheter. At the end of endoscopy the deflated catheter was inserted transorally and positioned at the EGJ. The endoscope was withdrawn and the balloon was inflated to 20 and $30 \mathrm{ml}$ distension volumes with a specially formulated conductive solution. To ensure proper bag placement, EndoFLIP measurements were monitored in real time by the display on the recording unit. An hourglass shape of the balloon was observed in all measurements with the narrowest CSA at the level of the esophagogastric junction. Measurements interrupted by balloon migration or esophageal peristalsis were repeated. After completing EGJ measurements, the EndoFLIP bag was deflated and removed. Immediately after the TIF procedure a second EndoFLIP measurement was performed in 25 patients. EGJ distensibility was assessed using the mean value over a 30 seconds dynamic measurement of the narrowest CSA and the corresponding intrabag pressure (narrowest CSA in $\mathrm{mm}^{2}$ /intra-bag pressure in $\mathrm{mmHg}$ ). 


\section{Transoral Incisionless Fundoplication (TIF procedure)}

TIF was performed using the EsophyX-2 device (EndoGastric Solutions, Inc., Redmond, WA, USA) according to the protocol as previously described. ${ }^{20}$ Patients were placed in the left lateral decubitus position after induction of general anesthesia. All procedures were performed by an experienced team of a gastroenterologist and a surgeon. The EsophyX device was inserted transorally over a flexible endoscope into the stomach. A retractor was anchored at the gastroesophageal junction and a hiatal hernia, if present, was reduced by returning the squamocolumnar junction to its natural position below the diaphragm using a built-in vacuum invaginator. A partial fundoplication was constructed through sequential retractions of tissue and tailored placement of multiple $\mathrm{H}$-shaped polypropylene fasteners circumferentially around the gastroesophageal junction to create a full thickness 240-330 degree circumference fundoplication (Figure 6.1).

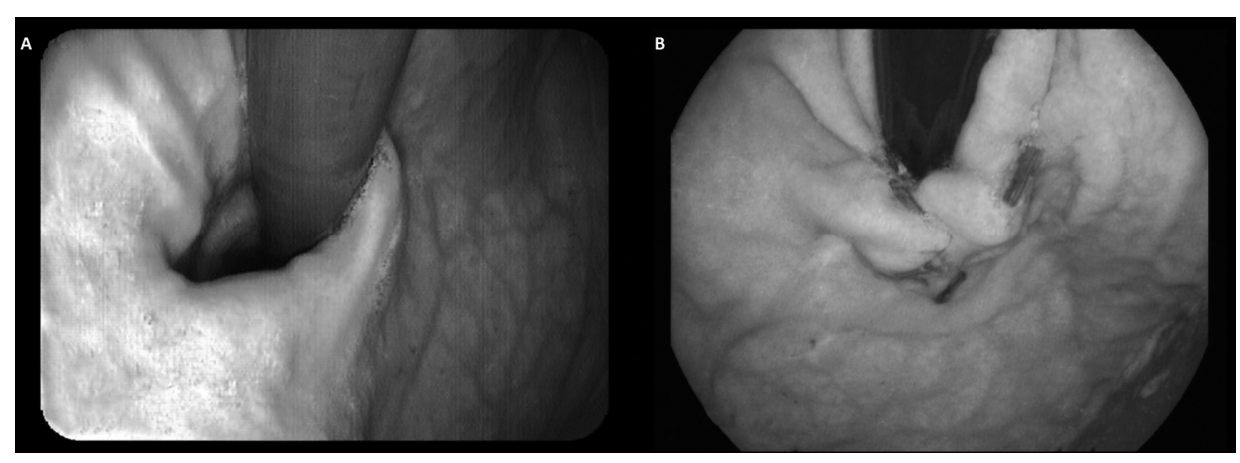

Figure 6.1 Gastroesophageal flap valve before (A) and after (B) Transoral Incisionless Fundoplication.

\section{Statistical analysis}

Data for continuous variables are expressed as median (interquartile range (IQR)). Statistical comparisons between preoperative and postoperative parameters were performed using Wilcoxon signed rank test; in case of proportions, McNemar's test was used. The Mann-Whitney $U$ test was used to assess differences between patients with a good or poor treatment outcome according to esophageal AET (objective outcome) or GERD-HRQL score (clinical outcome) at six months follow-up. Categorical variables were assessed using Chi-square test and correlations between EGJ distensibility and $\mathrm{pH}$-impedance parameters were calculated using Spearman's correlation coefficient. Multiple logistic regression analysis using age, gender, preoperative EGJ distensibility, preoperative acid exposure time, hiatal hernia size (in $\mathrm{cm}$ ) and gastroesophageal flap valve grade (I-IV) was performed to identify predictors of treatment success for the TIF procedure. Odds ratios (OR) are presented with $95 \%$ 
confidence intervals $(\mathrm{Cl})$. Values of $\mathrm{p}<0.05$ were considered significant. All statistical analyses were performed using commercially available computer software (PASW Statistics for Windows, Release Version 20.0.0, (C SPSS, Inc, Chicago, IL, USA).

\section{Results}

\section{Patient characteristics and symptom scores}

Of the total of 42 patients, 26 were male (62\%), mean age was 45 years (range 20-68 years) with a median BMI of $25.6 \mathrm{~kg} / \mathrm{m}^{2}\left(23.5-27.9 \mathrm{~kg} / \mathrm{m}^{2}\right)$. Assessment of EGJ distensibility using the EndoFLIP system was feasible in all but one patient due to incorrect placement of the EndoFLIP balloon and no adverse events occurred. Six months after TIF, symptom scores and $24 \mathrm{hr} \mathrm{pH}$-impedance monitoring results were available for 37 patients, whereas upper endoscopy was performed in 38 patients. Three patients were lost to follow-up, one patients had a defective $\mathrm{pH}$-measurement recording and one patient refused to undergo $\mathrm{pH}$-impedance monitoring due to a psychiatric disorder.

Six months after TIF, GERD symptoms had improved according to the reduction in GERD-HRQL scores (from $26(21-35)$ to 10 (4-20), p<0.01). Common use of acid suppressive medication was discontinued by $67 \%$ of patients, whereas $19 \%$ used PPIs occasionally and common use was present in $14 \%$.

\section{Endoscopy}

Prior to the TIF procedure, hiatal hernia was present in $28(67 \%)$ patients $(1 \mathrm{~cm}$ : 11 patients; $2 \mathrm{~cm}$ : 17 patients), most patients had gastroesophageal flap valve grade II $(60 \%)$ or III (31\%) and in $12(29 \%)$ patients esophagitis was described (grade A: 5 patients; grade B: 6 patients; grade C: 1 patient).

Six months after TIF, hiatal hernia was present in $15(39 \%)$ patients $(1 \mathrm{~cm}$ : 7 patients; $2 \mathrm{~cm}$ : 8 patients; $p=0.052$, compared to preoperative), $82 \%$ of patients had a normal Hill grade ( $p=0.022$, compared to preoperative) and $8(21 \%)$ patients had esophagitis (grade A: 3 patients; grade B: 4 patients; grade C: 1 patient). Preoperative esophagitis was cured in 8 patients, whereas 4 patients had persistent esophagitis and 4 patients had esophagitis de novo $(p=0.388)$.

\section{Objective treatment outcome}

Esophageal acid exposure time (AET) and number of reflux episodes were reduced at six months follow-up (Table 6.1). Normalization of esophageal AET was achieved in 21 patients whereas 16 patients had persistent abnormal AET. No difference was found in symptom scores between patients with normalized and persistent abnormal 
AET both preoperatively and at six months follow-up, and both patient groups had a significant reduction in symptom scores after TIF ( $p<0.01$, compared to preoperative). Six months after TIF, no difference was found in medication use between both patient groups ( $p=0.677$ ), whereas normal Hill grade was more often present in patients with normalized AET compared to patients with persistent pathological AET (100\% vs. 56\%, $\mathrm{p}<0.01)$.

Table 6.1 Twenty-four hour $\mathrm{pH}$-impedance parameters before and six months after Transoral Incisionless Fundoplication (TIF) ( $n=37)$.

\begin{tabular}{lccc}
\hline & Preoperative & $\begin{array}{c}\text { Six months } \\
\text { follow-up }\end{array}$ & p - value \\
\hline Acid exposure time total (\%) & $9.7(5.6-13.7)$ & $3.8(2.0-9.0)$ & $<0.01$ \\
Acid exposure time upright (\%) & $13.1(6.6-18.1)$ & $5.8(3.4-11.0)$ & $<0.01$ \\
Acid exposure time supine (\%) & $4.4(0.7-10.3)$ & $0.1(0.0-3.2)$ & $<0.05$ \\
Liquid reflux episodes (n) & $90(70-114)$ & $62(51-90)$ & $<0.01$ \\
Acid reflux episodes (n) & $59(43-76)$ & $36(21-50)$ & $<0.01$ \\
Proximal reflux episodes (n) & $45(31-53)$ & $23(14-37)$ & $<0.01$ \\
\hline
\end{tabular}

Values are presented as median (IQR)

Patients with normalization of AET at six months follow-up turned out to have a lower preoperative esophageal AET and lower preoperative EGJ distensibility compared to patients with persistent abnormal AET (Table 6.2). Six months after TIF, patients with normalized AET had lower numbers of liquid, acid and proximal reflux episodes compared to patients with persistent pathological AET (liquid: 57 (36-88) vs. 80 (60-129), p=0.012; acid: 31 (16-37) vs. 58 (35-68), $p<0.01$; proximal: 19 (8-27) vs. 41 $(21-56), p<0.01)$.

Table 6.2 Preoperative EndoFLIP parameters and preoperative acid exposure time in patients with normalized ( $\mathrm{pH}<4$ for $\leq 4 \%$ of time) and patients with persistent abnormal acid exposure time at six months follow-up.

\begin{tabular}{|c|c|c|c|}
\hline & $\begin{array}{c}\text { Normalized AET } \\
\quad(n=21)\end{array}$ & $\begin{array}{c}\text { Abnormal AET } \\
(n=16)\end{array}$ & $p$-value \\
\hline \multicolumn{4}{|c|}{ EGJ distensibility $\left(\mathrm{mm}^{2} / \mathrm{mmHg}\right)$} \\
\hline $20 \mathrm{ml}$ & $1.3(1.0-1.7)$ & $2.1(1.2-2.4)$ & 0.060 \\
\hline $30 \mathrm{ml}$ & $1.5(1.1-2.1)$ & $2.5(1.7-3.4)$ & $<0.05$ \\
\hline \multicolumn{4}{|c|}{$\operatorname{CSA}\left(\mathrm{mm}^{2}\right)$} \\
\hline $20 \mathrm{ml}$ & $21(20-24)$ & $25(21-33)$ & $<0.05$ \\
\hline $30 \mathrm{ml}$ & $29(22-38)$ & $38(26-54)$ & $<0.05$ \\
\hline \multicolumn{4}{|c|}{ Pressure (mmHg) } \\
\hline $20 \mathrm{ml}$ & $16(12-21)$ & $13(11-18)$ & NS \\
\hline $30 \mathrm{ml}$ & $20(15-26)$ & $15(14-21)$ & NS \\
\hline \multicolumn{4}{|c|}{ Acid exposure (\%) } \\
\hline Total & $7.6(5.3-10.6)$ & $11.8(9.3-16.8)$ & $<0.05$ \\
\hline Upright & $7.9(5.8-15.4)$ & $15.4(13.1-25.3)$ & $<0.05$ \\
\hline Supine & $4.3(0.3-10.3)$ & $5.0(1.2-12.9)$ & NS \\
\hline
\end{tabular}

$\mathrm{AET}=$ acid exposure time; $\mathrm{CSA}=$ cross sectional area; NS = not significant. Values are presented as median (IQR) 
Multiple logistic regression analysis, adjusted for age and gender, showed that preoperative EGJ distensibility (in $\mathrm{mm}^{2} / \mathrm{mmHg}$; OR $0.16,95 \% \mathrm{Cl} 0.03-0.78, \mathrm{p}=0.023$ ) and preoperative AET (in \%; OR 0.62, 95\% Cl 0.42-0.90, $\mathrm{p}=0.013$ ) were independent predictors for objective treatment success (i.e. normalization of acid exposure time) after TIF, whereas presence of preoperative hiatal hernia and gastroesophageal flap valve grade could not predict objective treatment outcome after TIF (Table 6.3). Since preoperative EGJ distensibility and preoperative AET are predictors of objective treatment outcome, we determined cut-off values to improve patient selection for the TIF procedure. To assess the best cut-off values, we evaluated the percentage of patients with normalized or persistent abnormal AET for different values. The best cut-off value for preoperative EGJ distensibility $(30 \mathrm{ml})$ was $2.3 \mathrm{~mm}^{2} / \mathrm{mmHg}$ as patients with a lower distensibility had normalized AET in $72 \%$ of cases, while patients with a higher preoperative distensibility maintained an abnormal AET in $82 \%$ of cases. The optimal cut-off value for preoperative AET was $11 \%$ (<11\%: $77 \%$ had normalized AET; >11\%: 79\% had persistent abnormal AET). Combining these results, we found that 15 out of 16 patients (94\%) meeting both criteria (EGJ distensibility at $30 \mathrm{ml}$ $<2.3 \mathrm{~mm}^{2} / \mathrm{mmHg}$ and acid exposure time $<11 \%$ ) had normalized AET at six months follow-up (Figure 6.2).

Table 6.3 Multiple logistic regression analysis to examine preoperative predictors for normalization of acid exposure time at six months follow-up.

\begin{tabular}{lccc}
\hline Parameters & OR & $\mathbf{9 5 \%} \mathbf{C l}$ & p-value \\
\hline Female gender (vs male gender) & 2.69 & $0.18-39.75$ & 0.471 \\
Age (years, continuous) & 0.99 & $0.89-1.10$ & 0.816 \\
EGJ distensibility ( $\mathrm{mm}^{2} / \mathrm{mmHg}$, continuous) & 0.16 & $0.03-0.78$ & 0.023 \\
Acid exposure time (\%, continuous) & 0.62 & $0.42-0.90$ & 0.013 \\
Normal gastroesophageal flap valve grade (vs abnormal flap valve grade) & 0.30 & $0.02-3.70$ & 0.345 \\
Absence of hiatal hernia (vs presence of small hiatal hernia) & 1.52 & $0.14-16.34$ & 0.731 \\
\hline
\end{tabular}

OR, odds ratio; $95 \% \mathrm{Cl}$, 95\% confidence interval; EGJ, esophagogastric junction

\section{Clinical treatment outcome}

In $59 \%$ of patients, an improvement in GERD-HRQL score of at least $50 \%$ was found whereas $41 \%$ of patients had less improvement or even deterioration in symptom scores at six months follow-up compared to baseline off PPIs. No differences were found between patients with and without a good clinical response with regard to EndoFLIP and $24 \mathrm{hr} \mathrm{pH}$-impedance parameters both preoperative and at six months follow-up. In contrast to objective treatment outcome, preoperative EGJ distensibility and preoperative AET were no predictors of clinical treatment outcome after TIF. 


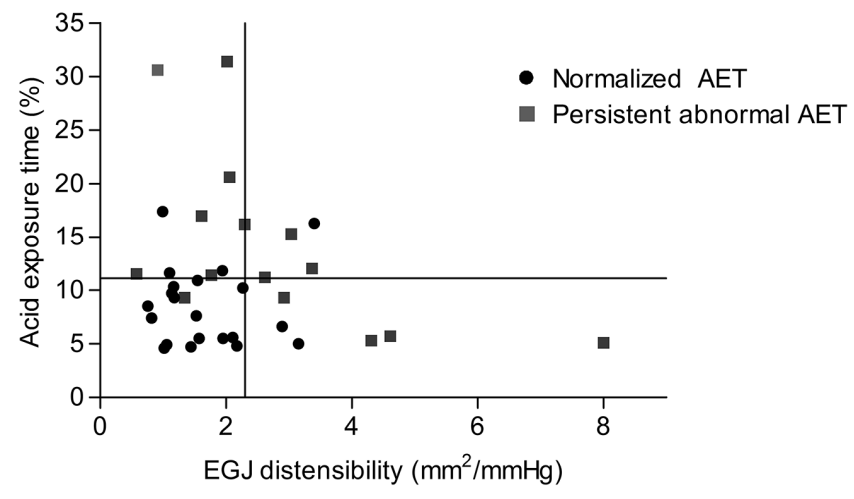

Figure 5.2 Relationship between preoperative EGJ distensibility and preoperative acid exposure time in patients with normalized acid exposure time $(n=21)$ and persistent abnormal acid exposure time $(n=16)$ at six months follow-up. Cut-off values: EGJ distensibility: $2.3 \mathrm{~mm} / \mathrm{mmHg}$; acid exposure time: $11 \%$.

\section{EGJ distensibility six months after TIF}

In 25 patients, EndoFLIP measurements were repeated direct postoperative and at six months follow-up. In the direct postoperative state, EGJ distensibility had decreased from $2.0(1.2-3.3)$ to $1.4(1.0-2.2) \mathrm{mm}^{2} / \mathrm{mmHg}(p=0.014)$ at the $30 \mathrm{ml}$ distension volume. At six months follow-up, no change in EGJ distensibility was found compared to baseline (Table 6.4). Although patients with normalized AET from this subgroup $(n=14)$ had a lower preoperative EGJ distensibility compared to patients with persistent abnormal AET (1.5 (1.1-2.3) vs. $\left.3.0(1.6-4.3) \mathrm{mm}^{2} / \mathrm{mmHg}, \mathrm{p}<0.05\right)$, both groups reached a comparable EGJ distensibility direct postoperative (1.6 (1.0-2.4) vs. $\left.1.3(0.9-2.1) \mathrm{mm}^{2} / \mathrm{mmHg}, \mathrm{p}=0.311\right)$ and at six months follow-up (2.0 (1.5-2.8) vs. 2.2 (1.4-4.0) $\left.\mathrm{mm}^{2} / \mathrm{mmHg}, \mathrm{p}=0.935\right)$.

Table 6.4 EndoFLIP parameters preoperative, direct postoperative and six months after Transoral Incisionless Fundoplication (TIF) ( $n=25)$.

\begin{tabular}{lccc}
\hline & Preoperative & Postoperative & Six months follow-up \\
\hline EGJ distensibility $\left(\mathrm{mm}^{2} / \mathrm{mmHg}\right)$ & $1.6(1.2-2.4)$ & $1.3(0.8-1.6)^{*}$ & $1.4(1.0-1.6)$ \\
$20 \mathrm{ml}$ & $2.0(1.2-3.3)$ & $1.4(1.0-2.2)^{*}$ & $2.2(1.5-3.0)$ \\
$30 \mathrm{ml}$ & & & \\
$\mathrm{CSA}\left(\mathrm{mm}^{2}\right)$ & $23(20-29)$ & $20(16-22)$ & $21(20-26)$ \\
$20 \mathrm{ml}$ & $35(27-49)$ & $36(21-42)$ & $41(27-60)$ \\
$30 \mathrm{ml}$ & & & \\
Pressure $(\mathrm{mmHg})$ & $14(11-17)$ & $16(14-19)^{*}$ & $16(15-19)$ \\
$20 \mathrm{ml}$ & $17(14-24)$ & $21(19-25)^{*}$ & $20(16-25)$ \\
$30 \mathrm{ml}$ & &
\end{tabular}

CSA, cross sectional area; EGJ, esophagogastric junction. Values are presented as median (IQR). ${ }^{*} \mathrm{p}<0.05$ (preoperative vs. postoperative). 


\section{EGJ distensibility and gastroesophageal reflux}

Preoperative EGJ distensibility $(30 \mathrm{ml})$ was weakly correlated with acid exposure time $(r=0.345, p<0.05)$ (Figure 6.3) and number of liquid $(r=0.389, p<0.05)$, acid $(r=0.325$, $p=0.053)$ and proximal reflux episodes $(r=0.474, p<0.01)$ at six months follow-up. In contrast, no correlation was found between preoperative EGJ distensibility and AET or number of reflux episodes prior to TIF. Furthermore, no association was found between objective treatment outcome (i.e. normalization of acid exposure time) and clinical treatment outcome (i.e. $\geq 50 \%$ improvement in GERD-HRQL score) after TIF $(p=0.868)$.

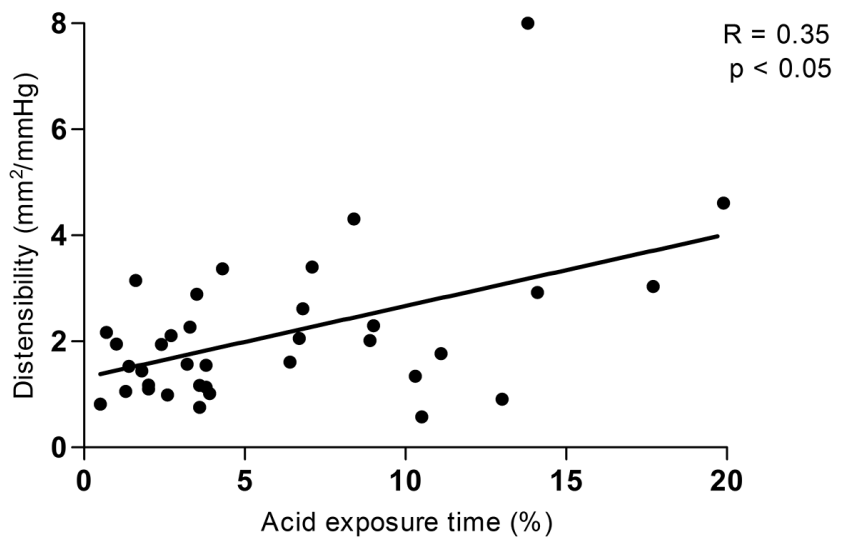

Figure 6.3 Correlation between preoperative EGJ distensibility $(30 \mathrm{ml})$ and acid exposure time at six months after Transoral Incisionless Fundoplication.

\section{Discussion}

Restoration of the incompetence of the EGJ by endoscopic or surgical techniques will allow symptom improvement, particularly in GERD patients unresponsive to drug therapy. One of the newer endoscopic approaches aimed to improve EGJ competence is Transoral Incisionless Fundoplication (TIF) with the Esophyx device. One hypothesis states that the TIF procedure restores the gastroesophageal junction by rotating the fundus around the esophagus and creating a partial fundoplication, thereby restoring EGJ distensibility with reduction in the volume of refluxate since flow across the EGJ is proportional to the opening diameter raised to the fourth power. ${ }^{12,20}$ The EndoFLIP technique is a new method to assess EGJ distensibility. A previous study described the safety of the use of the EndoFLIP before and direct after endoluminal fundoplication, and in the present study no complications occurred. ${ }^{21}$ 
In order to predict the responsiveness to TIF already in the preoperative state, we assessed EGJ distensibility using the EndoFLIP technique. We hypothesized that patients with low preoperative EGJ distensibility will have better EGJ competence compared to patients with high EGJ distensibility. Transoral Incisionless Fundoplication might be able to improve a slightly disturbed EGJ resulting in more competent functioning of the EGJ with consequently less esophageal acid exposure, whereas TIF might be unable to restore EGJ integrity in case of a severely impaired gastroesophageal junction. Our findings confirm the hypothesis by the outcome of a higher percentage of patients with normal gastroesophageal flap valve grade in patients with normalized AET compared to patients with persistent abnormal AET at six months follow-up. However, EGJ distensibility was not significantly different six months following TIF. The question arises why the decrease in AET and improvement in gastroesophageal flap valve grade is not associated with a change in EGJ distensibility at six months after TIF. This could be related to methodological issues of measuring EGJ distensibility. We have to consider that EndoFLIP results are mean values of a 30 second dynamic measurement. Distensibility of the EGJ is affected by several factors including tone and contractions of the esophagus, crural diaphragm, gastroesophageal flap valve grade and presence or absence of hiatal hernia. A possible limitation of the study is the fact that we measured EGJ distensibility before and after the TIF procedure while patients were under general anesthesia, whereas EndoFLIP measurements at six months follow-up were performed under conscious sedation. Although effects of general anesthesia on EGJ distensibility are considered negligible, we cannot exclude that this variable has affected muscle activity and consequently our data. ${ }^{22}$

A recent study demonstrated that EGJ distensibility assessed with the EndoFLIP technique was not able to distinguish GERD patients from healthy controls probably as result of the wide range of EGJ distensibility values in healthy controls. ${ }^{15,23,24}$ In addition, we hypothesized that preoperative EGJ distensibility or CSA would correlate with preoperative esophageal $A E T$, as the EGJ opening diameter determines reflux volume. ${ }^{12}$ However, no correlation was found between preoperative EGJ distensibility or CSA and the severity of GERD according to esophageal AET. Therefore, the EndoFLIP technique cannot be used to diagnose GERD or to grade the severity of GERD according to esophageal $A E T .^{23}$ Tucker et al. suggested that endoluminal measurement of EGJ distensibility with the EndoFLIP technique may not be able to reflect the action or interaction of the different structures of the complex EGJ which might be a potential explanation for the lack of correlation between both EGJ distensibility and AET prior to TIF. ${ }^{23}$ Moreover, Tucker et al. found an inverse correlation between EGJ distensibility, CSA and BMI. In the present study, BMI was not correlated to either preoperative EGJ distensibility or CSA, probably due to a lower median $\mathrm{BMI}$ with a narrower range in our participants. ${ }^{23}$ 
Although no difference was observed in EGJ distensibility following TIF, we did observe significant improvement in acid exposure time in $57 \%$ of the patients which is comparable to previous publications. ${ }^{8,25}$ Therefore, we suggest that changes in other factors like transient lower esophageal sphincter relaxations (TLESRs) and the acid pocket may contribute to the antireflux effect of the TIF procedure, and probably may explain the observed difference between patients with normalized AET and patients with persistent abnormal AET at six months follow-up. ${ }^{26,27}$

Patient selection is crucial in order to achieve optimal treatment outcome after TIF as normalization of AET was only present in $57 \%$ of patients. According to literature data, GERD patients with small hiatal hernia $(\leq 2 \mathrm{~cm})$ and normal gastroesophageal flap valve grade (Hill I-II) prior to TIF are considered best candidates. ${ }^{6,10}$ In the present study, gastroesophageal flap valve grade and presence or absence of hiatal hernia prior to TIF were not associated with normalization of AET after TIF. However, our results show that lower preoperative EGJ distensibility and moderately increased preoperative AET are associated with better objective treatment outcome after TIF (i.e. normalization of AET), whereas patients with high preoperative distensibility and prolonged AET appear to be better candidates for laparoscopic fundoplication. Based on our results, we suggest that patients with preoperative EGJ distensibility $<2.3 \mathrm{~mm}^{2} / \mathrm{mmHg}$ and preoperative $A E T<11 \%$ have the best objective treatment outcome. Recently, a comparable cutoff value of $10 \%$ for preoperative AET was suggested. ${ }^{25}$ However, it should be taken into account that this is the first study reporting on preoperative EGJ distensibility as predictive factor for objective treatment outcome of TIF. Our sample size is small and additional data are needed to confirm our results and optimize cut-off values.

Furthermore, we have to consider that an effective treatment for GERD is not only expected to reduce $A E T$, heal esophagitis and prevent the development of chronic complications, but also to relieve GERD symptoms. In contrast to objective treatment outcome, preoperative EGJ distensibility could not predict clinical treatment outcome after TIF. In addition, no association was found between objective (i.e. normalization of acid exposure time) and clinical (i.e. $\geq 50 \%$ improvement in GERD-HRQL score) treatment outcome. Although the first sham controlled trials are awaited, we hypothesize that one possible explanation for this apparent discrepancy might be the placebo effect as both patients with decreased and unaltered AET showed symptom improvement. ${ }^{28,29}$ In addition, Trad et al. described a lack of symptom improvement in patients with persistent pathological AET after acid suppressive therapy, whereas patients with persistent pathological AET after TIF had improved symptom scores. ${ }^{25}$ Moreover, we have to take into account that symptom perception in GERD patients is a complex pathophysiological mechanism influenced by multiple factors including reflux characteristics (i.e. acidity, proximal extent and composition of the refluxate), hypersensitivity and mucosal barrier function. ${ }^{30,31}$ In the present study, we evaluated reflux characteristics according to $24 \mathrm{hr} \mathrm{pH}$-impedance 
monitoring, whereas we did not objectively assess changes in (hyper)sensitivity and mucosal barrier function after TIF. This might at least partially explain the lack of association between objective and clinical treatment outcome after TIF, especially as previous studies described discrepancies between the severity of esophageal acid exposure and reported symptoms. ${ }^{30,32}$

In addition, our study had other limitations. We assessed the predictive effect of EGJ distensibility for medium term treatment outcome based on acid exposure time and symptom scores six months after TIF. Gastroesophageal reflux disease is however a chronic disorder and assessment of the predictive value of preoperative EGJ distensibility for long-term follow-up is necessary. Furthermore, we have to take into account practical and financial aspects of the EndoFLIP technique especially as assessment of AET is part of the preoperative work-up of GERD patients. Measurement of EGJ distensibility with the EndoFLIP device is a relatively new method and the precise role of this technique in the diagnosis and management of upper gastrointestinal diseases is still unknown. Therefore, the EndoFLIP is not a routine technique and only available in a limited number of $\mathrm{Gl}$ centers, despite the fact that the device is commercially available. Although EndoFLIP offers the possibility to acquire assessment of EGJ distensibility in a more practical method compared to barostat, we have to consider that measurement of EGJ distensibility with the EndoFLIP technique remains an invasive procedure which may be associated with discomfort in patients. ${ }^{33}$ Finally, we have to take into account additional costs of this technique as both the mobile recording device and single-use catheters are expensive.

Taken our results and arguments into consideration, no evidence has been provided for the use of the EndoFLIP technique in GERD patients either in the preoperative diagnostic work-up or in the post-procedure evaluation. However, we cannot exclude that the EndoFLIP device may have additional value in the follow-up of patients with postoperative symptoms (e.g. dysphagia) after antireflux procedures or for assessment of treatment efficacy in achalasia patients. ${ }^{24}$

In conclusion, preoperative esophagogastric junction (EGJ) distensibility and preoperative esophageal acid exposure time were independent predictors for objective treatment outcome after Transoral Incisionless Fundoplication in GERD patients according to esophageal acid exposure time, whereas preoperative EGJ distensibility was not able to predict subjective, clinical treatment outcome. Moreover, EGJ distensibility was not different at six months follow-up compared to baseline. According to our data, we conclude that the EndoFLIP technique has no added value either in the preoperative diagnostic work-up or in the post-procedure evaluation of endoluminal antireflux therapy. 


\section{References}

1. Vakil N. Review article: the role of surgery in gastro-oesophageal reflux disease. Aliment Pharmacol Ther. 2007;25(12):1365-72.

2. Lundell L, Miettinen P, Myrvold HE, Hatlebakk JG, Wallin L, Malm A, et al. Seven-year follow-up of a randomized clinical trial comparing proton-pump inhibition with surgical therapy for reflux oesophagitis. Br J Surg. 2007;94(2):198-203.

3. Freston JW, Triadafilopoulos G. Review article: approaches to the long-term management of adults with GERD-proton pump inhibitor therapy, laparoscopic fundoplication or endoscopic therapy? Aliment Pharmacol Ther. 2004;19 Suppl 1:35-42.

4. Louis H, Deviere J. Ensocopic-endoluminal therapies. A critical appraisal. Best Pract Res Clin Gastroenterol. 2010;24(6):969-79.

5. Cadiere GB, Van Sante N, Graves JE, Gawlicka AK, Rajan A. Two-year results of a feasibility study on antireflux transoral incisionless fundoplication using EsophyX. Surg Endosc. 2009;23(5):957-64.

6. Cadiere GB, Buset M, Muls V, Rajan A, Rosch T, Eckardt AJ, et al. Antireflux transoral incisionless fundoplication using EsophyX: 12-month results of a prospective multicenter study. World J Surg. 2008;32(8):1676-88.

7. Frazzoni M, Conigliaro R, Manta R, Melotti G. Reflux parameters as modified by EsophyX or laparoscopic fundoplication in refractory GERD. Aliment Pharmacol Ther. 2011;34(1):67-75.

8. Bell RC, Mavrelis PG, Barnes WE, Dargis D, Carter BJ, Hoddinott KM, et al. A prospective multicenter registry of patients with chronic gastroesophageal reflux disease receiving transoral incisionless fundoplication. J Am Coll Surg. 2012;215(6):794-809.

9. Testoni PA, Vailati C, Testoni S, Corsetti M. Transoral incisionless fundoplication (TIF 2.0) with EsophyX for gastroesophageal reflux disease: long-term results and findings affecting outcome. Surg Endosc. 2012;26(5):1425-35.

10. Witteman BP, Strijkers R, de Vries E, Toemen L, Conchillo JM, Hameeteman W, et al. Transoral incisionless fundoplication for treatment of gastroesophageal reflux disease in clinical practice. Surg Endosc. 2012;26(11):3307-15.

11. Ghosh SK. Biomechanics of the esophagogastric junction in gastroesophageal reflux disease. Curr Gastroenterol Rep. 2008;10(3):246-51.

12. Pandolfino JE, Shi G, Curry J, Joehl RJ, Brasseur JG, Kahrilas PJ. Esophagogastric junction distensibility: a factor contributing to sphincter incompetence. Am J Physiol Gastrointest Liver Physiol. 2002;282(6):G1052-8.

13. Pandolfino JE, Curry J, Shi G, Joehl RJ, Brasseur JG, Kahrilas PJ. Restoration of normal distensive characteristics of the esophagogastric junction after fundoplication. Ann Surg. 2005;242(1):43-8.

14. Blom D, Bajaj S, Liu J, Hofmann C, Rittmann T, Derksen T, et al. Laparoscopic Nissen fundoplication decreases gastroesophageal junction distensibility in patients with gastroesophageal reflux disease. J Gastrointest Surg. 2005;9(9):1318-25.

15. Kwiatek MA, Pandolfino JE, Hirano I, Kahrilas PJ. Esophagogastric junction distensibility assessed with an endoscopic functional luminal imaging probe (EndoFLIP). Gastrointest Endosc. 2010;72(2):272-8.

16. Velanovich V. The development of the GERD-HRQL symptom severity instrument. Dis Esophagus. 2007;20(2):130-4.

17. Rothstein R, Filipi C, Caca K, Pruitt R, Mergener K, Torquati A, et al. Endoscopic full-thickness plication for the treatment of gastroesophageal reflux disease: A randomized, sham-controlled trial. Gastroenterology. 2006;131(3):704-12.

18. Hill LD, Kozarek RA. The gastroesophageal flap valve. J Clin Gastroenterol. 1999;28(3):194-7.

19. McMahon BP, Frokjaer JB, Kunwald P, Liao D, Funch-Jensen P, Drewes AM, et al. The functional lumen imaging probe (FLIP) for evaluation of the esophagogastric junction. Am J Physiol Gastrointest Liver Physiol. 2007;292(1):G377-84.

20. Bell RC, Cadiere GB. Transoral rotational esophagogastric fundoplication: technical, anatomical, and safety considerations. Surg Endosc. 2011;25(7):2387-99. 
21. Hoppo T, McMahon BP, Witteman BP, Kraemer SJ, O'Rourke RW, Gravesen F, et al. Functional lumen imaging probe to assess geometric changes in the esophagogastric junction following endolumenal fundoplication. J Gastrointest Surg. 2011;15(7):1112-20.

22. Nathanson LK, Brunott N, Cavallucci D. Adult esophagogastric junction distensibility during general anesthesia assessed with an endoscopic functional luminal imaging probe (EndoFLIP(R)). Surg Endosc. 2012;26(4):1051-5.

23. Tucker E, Sweis R, Anggiansah A, Wong T, Telakis E, Knowles K, et al. Measurement of esophagogastric junction cross-sectional area and distensibility by an endolumenal functional lumen imaging probe for the diagnosis of gastroesophageal reflux disease. Neurogastroent Motil. 2013;25(11):90410.

24. Rohof WO, Hirsch DP, Kessing BF, Boeckxstaens GE. Efficacy of treatment for patients with achalasia depends on the distensibility of the esophagogastric junction. Gastroenterology. 2012;143(2):328-35.

25. Trad KS, Barnes WE, Simoni G, Shughoury AB, Mavrelis PG, Raza M, et al. Transoral incisionless fundoplication effective in eliminating GERD symptoms in partial responders to proton pump inhibitor therapy at 6 months: The TEMPO Randomized Clinical Trial. Surg Innov. 2015;22(1):26-40.

26. Rinsma NF, Smeets FG, Bruls DW, Kessing BF, Bouvy ND, Masclee AA, et al. Effect of transoral incisionless fundoplication on reflux mechanisms. Surg Endosc. 2013.

27. Herbella FA, Vicentine FP, Del Grande JC, Patti MG. Postprandial proximal gastric acid pocket in patients after laparoscopic Nissen fundoplication. Surg Endosc. 2011;25(10):3198-201.

28. Hogan WJ. Clinical trials evaluating endoscopic GERD treatments: is it time for a moratorium on the clinical use of these procedures? Am J Gastroenterol. 2006;101(3):437-9.

29. Pearl JP, Marks JM. Endolumenal therapies for gastroesophageal reflux disease: are they dead? Surg Endosc. 2007;21(1):1-4.

30. Weijenborg PW, Bredenoord AJ. How reflux causes symptoms: Reflux perception in gastroesophageal reflux disease. Best Pract Res Cl Ga. 2013;27(3):353-64.

31. Harnett KM, Rieder F, Behar J, Biancani P. Viewpoints on Acid-induced inflammatory mediators in esophageal mucosa. J Neurogastroenterol Motil. 2010;16(4):374-88.

32. Stein HJ, Hoeft S, DeMeester TR. Functional foregut abnormalities in Barrett's esophagus. J Thorac Cardiovasc Surg. 1993;105(1):107-11.

33. Verlaan T, Rohof WO, Bredenoord AJ, Eberl S, Rosch T, Fockens P. Effect of peroral endoscopic myotomy on esophagogastric junction physiology in patients with achalasia. Gastrointest Endosc. 2013;78(1):39-44. 


\section{Chapter 7}

\section{Endoscopic botulinum toxin for gastroparesis: results of a retrospective series}

F.G.M. Smeets*, D. Strijbos*, D. Keszthelyi, C.V. Hoge, J.W. Kruimel, J.M. Conchillo, A.A.M. Masclee *both authors contributed equally Gastrointest. Disord. 2019;1(1):164-175 


\section{Abstract}

\section{Objective}

Beneficial effects of pyloric botulinum toxin injection have been described in a subgroup of gastroparesis patients. Our aim is to evaluate whether clinical, manometric and/or scintigraphic parameters are able to predict treatment outcome.

\section{Methods}

Forty patients (67\% female, age 49 (36-56) years) with decompensated gastroparesis treated with botulinum toxin were included in this retrospective analysis. Objective parameters were high-resolution antroduodenal manometry, gastric emptying and weight change. Subjective treatment outcome was assessed with a Global Physician Assessment Scale. Binary logistic regression analysis was performed to identify predictors for treatment outcome.

\section{Results}

Fourteen patients (35\%) were symptom-responders, and $65 \%$ of patients were weight-responders. For both subjective and objective treatment outcome, no differences were found in manometric and scintigraphic variables between responders and non-responders. Neither clinical, nor manometric or scintigraphic variables could predict subjective and objective treatment outcome.

\section{Conclusions}

Symptom improvement is achieved in a subgroup of gastroparesis patients treated with endoscopic pyloric botulinum toxin. Although the majority of patients was able to maintain their baseline weight at short-term follow-up, a substantial group of patients needed nutritional interventions on long-term follow-up. However, none of the demographic, clinical, scintigraphic or antroduodenal manometry variables were able to predict either subjective or objective treatment outcome. 


\section{Introduction}

Gastroparesis is defined by delayed gastric emptying in the absence of a mechanical obstruction. It is associated with a wide variety of symptoms including nausea, early satiation, postprandial fullness, vomiting, upper abdominal pain and bloating. ${ }^{1-5}$ Common etiological factors are diabetes mellitus, vagal nerve injury after gastric surgery although in the majority the cause remains unknown (i.e. idiopathic gastroparesis). Pathophysiological mechanisms include impaired gastric accommodation, antral hypomotility, disordered antroduodenal coordination, pyloric dysfunction and excessive inhibitory duodenogastric feedback. ${ }^{6-9}$

Despite the increasing knowledge about pathophysiological mechanisms, management remains challenging. Initial treatment consists of dietary interventions and use of prokinetic agents. ${ }^{10,11}$ In case of refractory symptoms, more invasive (endoscopic) and nutritional interventions are considered, including pyloric botulinum toxin. ${ }^{12,13}$

Botulinum toxin $\mathrm{A}$ is a neurotoxic protein produced by the bacterium Clostridium botulinum which inhibits the release of acetylcholine at the neuromuscular junction. Pyloric botulinum injection decreases smooth muscle contractility and pyloric pressures. ${ }^{14-16}$ Two small randomized placebo-controlled trials evaluated the effect of botulinum toxin in gastroparesis patients. Although both symptoms and gastric emptying improved after treatment with botulinum toxin, no significant difference was seen compared to placebo. ${ }^{17,18}$ Consequently, treatment with botulinum toxin is currently not recommended by the American College of Gastroenterology (ACG). ${ }^{12}$ On the other hand, pyloric botulinum toxin is still regularly used in clinical practice because alternative treatment options are lacking. Previously it has been hypothesized that treatment with botulinum toxin might be beneficial for at least a subgroup of gastroparesis patients. Gender, lower age and idiopathic gastroparesis proved to be clinical predictors for symptom improvement in one study, which findings were not reproduced in other studies. ${ }^{19,20}$ Objective predictors for treatment outcome are currently not available. Therefore, we aimed to evaluate whether objective, manometric and/or scintigraphic parameters, in addition to clinical characteristics, are able to predict treatment outcome in patients with gastroparesis treated with pyloric botulinum toxin. We hypothesized that more severe delayed gastric emptying and/or antral hypomotility would be associated with a less favorable treatment outcome with both persistent symptoms and inability to maintain body weight. 


\section{Methods}

\section{Design, setting and participants}

After institutional review board approval, we used patient medical records and our hospital's pharmacy database to retrospectively identify all patients with decompensated gastroparesis (Grade III, refractory to dietary interventions and medical therapy) who received botulinum toxin for treatment of gastroparesis between 2008 and 2016 in the Maastricht University Medical Center (MUMC+), a tertiary referral center. ${ }^{21,22}$

\section{Scintigraphy}

Scintigraphy at MUMC+ was performed according to standard procedures. ${ }^{23,24}$ Medication affecting gastrointestinal tract motility was stopped at least 3 days prior to the study. After an overnight fast a standard meal was given to the patient, consisting of $100 \mathrm{ml}$ egg whites mixed with ${ }^{99 \mathrm{~m}} \mathrm{Tc}$ nanocolloïd (microwaved for $2 \mathrm{~min}$ ), 1 slice of bread with $15 \mathrm{~g}$ jam, and $120 \mathrm{ml}$ radiolabelled $\left({ }^{111}\right.$ In DTPA) water (118 kcal, $12 \%$ fat, $77 \%$ carbohydrates, $11 \%$ protein). Patients were instructed to finish this meal within $10 \mathrm{~min}$. Immediately after meal ingestion, images were obtained $(\mathrm{t}=0)$ as well as 60 , 120 , and 240 min after the meal.

\section{High resolution antroduodenal manometry}

Subjects underwent high-resolution antroduodenal manometry after an overnight fast of at least 8 hours. Medication affecting gastrointestinal tract motility was stopped 3 days prior to the study. A solid-state high-resolution catheter consisting of 36 transducers, spaced at 1-cm intervals, was used (Unisensor AG, Attikon, Switzerland). The catheter was advanced across the pylorus into the duodenum under fluoroscopic guidance with approximately 12 pressure sensors located into the stomach. After correct placement, the catheter leads were connected to the MMS working station (MMS Solar GI HRM, Medical Measurement Systems, Enschede, The Netherlands). The sampling frequency was $10 \mathrm{~Hz}$. After $30 \mathrm{~min}$ recording in fasting conditions, subjects were given a standardized meal consisting of one scrambled egg, two slices of white bread with $5 \mathrm{mg}$ of margarine and $150 \mathrm{ml}$ of water ( $283 \mathrm{kcal}, 41.2 \%$ fat, $17.1 \%$ carbohydrates, $41.6 \%$ protein). After ingestion of the meal, data were recorded for 6 hours, during which patients were not allowed to eat or drink.

\section{Endoscopic pyloric botulinum toxin injection}

After an overnight fast, patients underwent a standard upper gastrointestinal endoscopy under conscious sedation (midazolam 2.5-5 mg intravenously). Botulinum 
toxin (abobotulinum toxin A, Dysport, Ispen Farmaceutica bv) at least 500IE diluted in $2.5 \mathrm{ml}$ of saline, was injected in $0.6 \mathrm{ml}$ aliquots in each quadrant of the pylorus using a sclerotherapy needle. The proposed conversion rate between Botox (onabotulinum toxin A) and Dysport seems to be 1:3, which means that one unit Botox is comparable to 3 units of Dysport.

\section{Outcomes}

Primary objective was to assess the relation between variables (scintigraphy and antroduodenal manometry) and both subjective and objective outcome parameters.

Subjective treatment outcome was assessed with a Global Physician Assessment Scale (GPA 1: complete relief; GPA 2: marked relief; GPA 3: moderate relief; GPA 4: slight relief; GPA 5: no relief; GPA 6: worsening symptoms), based on the clinical effect reported by the patient. ${ }^{25,26}$ GPA was measured four weeks after botulinum toxin treatment or at the first control visit after treatment. Reported complete and marked relief (GPA 1-2) was considered as successful treatment. In order to improve the accuracy of the assessment of subjective treatment outcome, two reviewers independently evaluated the patients' chart and defined GPA scores with a concordance rate of $98 \%$. Individual symptoms of gastroparesis (e.g. vomiting, nausea, postprandial fullness) were not assessed separately.

Change in weight after treatment with botulinum toxin injection was used as objective treatment outcome. Weight change was measured four weeks after botulinum toxin treatment or at the first control visit after treatment. Due to the small number of patients with weight gain after treatment, we considered both weight gain and maintenance of baseline weight as treatment success. Patients with weight loss were considered as non-responders. In addition, weight change before endoscopic treatment with botulinum toxin was described as either (1) stable weight, (2) <10kg weight loss or $(3) \geq 10 \mathrm{~kg}$ weight loss (measured approximately within six months prior to pyloric botulinum therapy).

\section{Data analysis}

\section{Scintigraphy}

For the four-hour solid phase gastric emptying scintigraphy, total gastric counts were normalized to $100 \%$ for $\mathrm{t}=0$ ( $0 \mathrm{~min}$; image directly after meal ingestion). The percentage of contents remaining in the stomach was reported after 60, 120 and $240 \mathrm{~min}$. Normal gastric retention values were $53-79 \%$ for $60 \mathrm{~min}, 16-37 \%$ for $120 \mathrm{~min}$, and $0-4 \%$ for $240 \mathrm{~min}$. Normal values for solid half emptying time were 64-103 min. 
Fifteen patients were diagnosed with gastroparesis by a short scintigraphy test (120 min, $\mathrm{n}=14$ ), performed at the referring hospitals and/or ${ }^{13} \mathrm{C}$-octanoic acid breath test $(n=1)$. As comparison of different tests and/or study protocols is not possible, we only report quantitative results of the recommended four-hour solid phase gastric emptying scintigraphy $(n=25)$.

Patients were diagnosed with gastroparesis in case of (a) 4 hour gastric retention of solids above the upper limit of normal, or (b) prolonged gastric half emptying time according to 4 hour scintigraphy, short scintigraphy or breath test.

\section{High resolution antroduodenal manometry}

After elimination of artefacts, the location of the individual pressure transducers was determined visually. ${ }^{27,28}$ As a result of displacement of the catheter in several subjects, the localization of the individual pressure recordings had to be adjusted during analysis of the manometric data.

An increase in pressure of at least $10 \mathrm{mmHg}$ compared to baseline with a minimum duration of 1 second was considered a valid contraction and these contractions were included in automatic analyses. Although the postprandial period is defined as the interval between start of the meal until the occurrence of the first duodenal phase III episode, we defined the postprandial period in all subjects as 120 min after intake of the standardized meal since not all subjects had a phase III episode at the end of the study. ${ }^{29}$ Moreover, a postprandial period of $120 \mathrm{~min}$ seems to be acceptable as occurrence of phase III within 60-90 min after ingestion of a meal provides evidence for failure to induce a fed pattern in subjects. ${ }^{30,31}$

During the postprandial period, we determined frequency of valid contractions (contractions per $\mathrm{min})$, contraction amplitude $(\mathrm{mmHg})$ and motility index of antral pressure waves $(\mathrm{mmHg})$. Motility index (MI) was calculated using the formula: $\mathrm{MI}=\mathrm{In}$ ([number of waves $x \Sigma$ amplitude] +1 ) in the two pressure sensors located proximally to the manometrically and fluoroscopically defined pylorus, as previously described. Antral hypomotility was defined as a postprandial antral motility index $<13.67 \mathrm{mmHg}^{32}$

\section{Statistical methods}

Data analysis was performed using SPSS for Windows, version 23 (IBM Corporation). Non-parametric tests were used as several variables had no normal distribution.

Data are presented as frequencies for categorical variables, and as median (interquartile range; IQR) for continuous variables. The Chi ${ }^{2}$-test or Fisher's exact test (in case of small samples) was used to assess differences between categorical variables, whereas the Mann Whitney $U$ test and Kruskal Wallis test were used for continuous variables. The Spearman correlation coefficient $(R)$ was used to assess correlations between variables, and a binary univariate logistic regression analysis was 
performed to identify predictors for treatment outcome (Odds ratio, 95\%Cl). Patient characteristics, manometric and scintigraphic variables were used as potential predictors. All statistical tests were two-sided, with a significance level of 0.05 .

\section{Results}

\section{Patient characteristics}

Between 2008 and 2016, 40 patients (67\% female, median age 49 (36-56) years) with decompensated gastroparesis were treated with endoscopic botulinum toxin injection. Baseline characteristics are shown in Table 7.1.

Table 7.1 Baseline characteristics.

\begin{tabular}{lc}
\hline Characteristics & Median (IQR $\left.{ }^{\ddagger}\right)$ \\
\hline Demographic parameters & $49(36-56)$ \\
Age (years) & $168(164-178)$ \\
Length (cm) & $66.3(57-80)$ \\
Weight before botulinum toxin (kg) & $23.7(20.2-26.6)$ \\
BMI before botulinum toxin (kg/m²) & $\mathrm{N}(\%)$ \\
Etiology & $17(42.5 \%)$ \\
$\quad$ Idiopathic & $13(32.5 \%)$ \\
$\quad$ Diabetic & $10(25 \%)$ \\
$\quad$ Post-surgical & $8.4(7.5-10.9)$ \\
HbA1C* (\%) & \\
Gastric emptying parameters & $146(127-188)$ \\
T50 solid (min; normal 64-103 min) & $89(82-91)$ \\
Stasis 60 min (\%; normal 53-79\%) & $61(53-76)$ \\
Stasis 120 min (\%; normal 16-37\%) & $17(2-31)$ \\
Stasis 240 min (\%; normal 0-4\%) & \\
Antroduodenal manometric parameters & \\
Antral frequency (contractions/min) & $1.27(1.02-1.75)$ \\
Antral amplitude (mmHg) & $58(38-77)$ \\
Antral Motility Index (MI) (mmHg) & $13.59(12.57-14.81)$ \\
Antral hypomotility $(\mathrm{n}, \%)$ & $14(53.8 \%)$ \\
\hline
\end{tabular}

$\S$ Antral hypomotility: postprandial motility index $<13.67 \mathrm{mmHg}$; ${ }^{\mathrm{BMI}}$ : body mass index; * HbA1c: glycated hemoglobin $(n=11) ;{ }^{\ddagger}$ IQR: interquartile range.

Antroduodenal high-resolution manometry (ADM) was performed in 35 of the 40 patients. (Figure 7.1) Twenty-six ADM measurements were eligible for analysis as predictive factor. Ineligibility was due to inability to determine correct positioning of the catheter on the basis of manometric recording $(n=4)$, and performance of ADM after previous treatment with botulinum toxin $(n=5)$. 


\section{Subjective treatment outcome}

Based on the GPA score, 14 patients (35\%) were considered as symptom-responders whereas 26 patients (65\%) were non-responders (GPA 1: $2.5 \%$, GPA 2: 32.5\%, GPA 3: 2.5\%, GPA 4: 7.5\%, GPA 5: 55\%, GPA 6: 0\%). No correlations were found between subjective treatment outcome and manometric or scintigraphic variables. No differences were found in manometric and scintigraphic variables between symptomresponders and symptom-non-responders (data not shown).

The method of gastric emptying assessment, either (a) 4 hour gastric emptying scintigraphy $(n=25)$, or (b) short gastric emptying scintigraphy or breath test $(n=15)$, was not associated with subjective treatment outcome (Fisher exact test: $p=0.91$ ). In addition, no differences were found in subjective treatment outcome between patients diagnosed with gastroparesis on the basis of (a) increased 4 hour gastric retention (40\%), or (b) prolonged gastric half emptying time $(32 \%$, Fisher exact test: $\mathrm{p}=0.74)$.

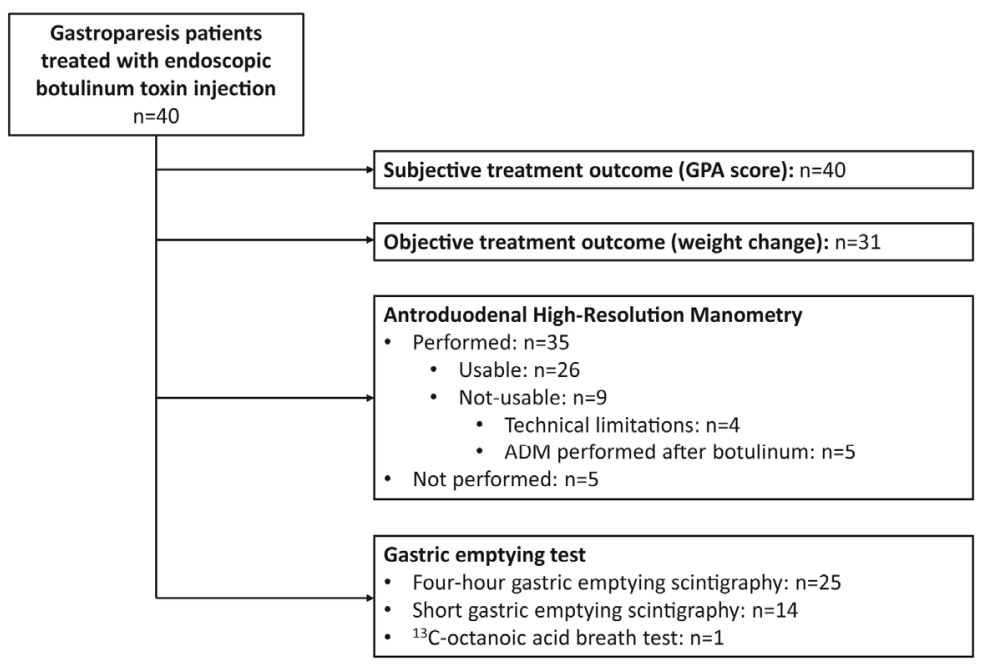

Figure 7.1 Flow chart of included gastroparesis patients.

\section{Objective treatment outcome}

Before treatment with botulinum toxin, 12 patients had $<10 \mathrm{~kg}$ weight loss whereas 14 patients had $\geq 10 \mathrm{~kg}$ weight loss. Seven patients had a stabile weight and no information was available for seven patients.

Weight change outcomes were available for 31 patients. Seventeen patients maintained their baseline weight (55\%); three patients showed weight gain (10\%) and weight loss occurred in eleven patients (35\%; two symptom-responders and nine 
symptom-non-responders). Overall, weight and BMI decreased after botulinum toxin treatment (weight: $67.0(58.0-80.0)$ to 65.0 (57.0-78.0) $\mathrm{kg}, \mathrm{p}=0.03$; BMI: 23.9 $(20.5-26.5)$ to $\left.23.0(19.8-26.8) \mathrm{kg} / \mathrm{m}^{2}, \mathrm{p}=0.01\right)$, and this decrease was most apparent in the symptom non-responder group (Table 7.2, data available for 30 patients). BMI before and after botulinum toxin treatment was lower in symptom non-responders compared to symptom-responders $(p<0.05$; Table 7.2$)$. In addition, a negative correlation was found between symptom scores and $\mathrm{BMI}$ after botulinum toxin $(R=-0.42, p=0.02)$.

Subjective and objective treatment outcome was available for 31 patients. Seven patients were considered both subjective and objective responders (23\%), whereas 12 patients were both subjective and objective non-responders (42\%). The remaining patients were either only subjective $(6 \%)$ or objective responders $(29 \%)$. No association was found between subjective and objective treatment outcome (Fisher exact test: $p=0.67)$. In addition, no significant differences were found in patient characteristics, manometric and scintigraphic variables between weight-responders and weight-non-responders.

The method of gastric emptying assessment, either (a) 4 hour gastric emptying scintigraphy $(n=25)$, or (b) short gastric emptying scintigraphy or breath test $(n=15)$, was not associated with objective treatment outcome (Fisher exact test: $p=0.71$ ). In addition, no differences were found in objective treatment outcome between patients diagnosed with gastroparesis on the basis of (a) increased 4 hour gastric retention $(58 \%)$, or (b) prolonged gastric half emptying time (68\%, Fisher exact test: $p=0.71)$.

Table 7.2 BMI in symptom responders vs. symptom non-responders.

\begin{tabular}{lccc}
\hline & $\begin{array}{c}\text { BMI before botulinum toxin } \\
\text { (median; IQR) }\end{array}$ & $\begin{array}{c}\text { BMI after botulinum toxin } \\
\text { (median; IQR) }\end{array}$ & p-value \\
\hline Overall $(\mathrm{n}=30)$ & $23.9(20.5-26.5)$ & $23.0(19.8-26.8)$ & 0.01 \\
Responders $(\mathrm{n}=8)$ & $27.6(24.3-30.2)$ & $27.6(22.9-30.2)$ & 0.67 \\
Non-responders $(\mathrm{n}=22)$ & $22.6(19.3-24.9)$ & $21.8(19.3-24.3)$ & $<0.01$ \\
\hline
\end{tabular}

BMI: Body Mass Index

\section{Predictors}

Age, gender, weight and BMI before botulinum toxin treatment, dose of botulinum toxin and etiology of gastroparesis could not predict either subjective (Table 7.3) or objective treatment outcome (Table 7.4). In addition, baseline parameters from scintigraphy and antroduodenal manometry were not able to predict subjective (Table 7.3) or objective (Table 7.4) treatment outcome. 
Table 7.3 Predictors for subjective treatment outcome* according to univariate binary logistic regression analysis.

\begin{tabular}{lcc}
\hline Parameters & Odds ratio (95\% Cl) & p-value \\
\hline Age & $1.01(0.96-1.06)$ & 0.62 \\
Gender & $1.25(0.32-4.94)$ & 0.75 \\
Etiology & & \\
$\quad$ Idiopathic vs. non-idiopathic & $0.63(0.17-2.32)$ & 0.48 \\
$\quad$ Diabetic vs. non-diabetic & $0.80(0.20-3.16)$ & 0.75 \\
$\quad$ Post-surgical vs. non post-surgical & $2.67(0.48-14.79)$ & 0.26 \\
Dose of botulinum toxin & $1.00(1.00-1.00)$ & 0.62 \\
Antral contraction frequency & $0.66(0.15-2.99)$ & 0.59 \\
Antral contraction amplitude & $0.99(0.96-1.03)$ & 0.71 \\
Antral motility index & $0.65(0.36-1.17)$ & 0.15 \\
Antral hypomotility & $5.25(0.50-54.91)$ & 0.17 \\
Gastric half emptying time (solids) & $1.00(0.99-1.01)$ & 0.85 \\
Gastric retention at 120 min & $1.01(0.94-1.08)$ & 0.84 \\
Gastric retention at 240 min & $1.00(0.95-1.04)$ & 0.81 \\
Weight before botulinum toxin & $1.04(0.98-1.10)$ & 0.24 \\
BMI before botulinum toxin & $1.15(0.97-1.36)$ & 0.11 \\
\hline
\end{tabular}

BMI: body mass index. *Subjective treatment outcome: complete or marked symptom relief (GPA 1-2) after botulinum toxin treatment.

Table 7.4 Predictors for objective treatment outcome* according to univariate binary logistic regression analysis.

\begin{tabular}{lcc}
\hline Parameters & Odds ratio (95\% Cl) & p-value \\
\hline Age & $1.02(0.96-1.08)$ & 0.56 \\
Gender & $0.40(0.08-1.90)$ & 0.25 \\
Etiology & & \\
$\quad$ Idiopathic vs. non-idiopathic & $2.23(0.50-10.00)$ & 0.30 \\
$\quad$ Diabetic vs. non-diabetic & $0.33(0.06-1.97)$ & 0.23 \\
$\quad$ Post-surgical vs. non post-surgical & $1.13(0.21-5.97)$ & 0.89 \\
Dose of botulinum toxin & $1.00(1.00-1.00)$ & 0.92 \\
Antral contraction frequency & $0.47(0.10-2.11)$ & 0.32 \\
Antral contraction amplitude & $1.02(0.99-1.06)$ & 0.23 \\
Antral motility index & $0.98(0.56-1.69)$ & 0.94 \\
Antral hypomotility & $0.78(0.04-14.75)$ & 0.87 \\
Gastric half emptying time (solids) & $0.99(0.98-1.00)$ & 0.36 \\
Gastric retention at 120 min & $0.96(0.89-1.05)$ & 0.38 \\
Gastric retention at 240 min & $0.97(0.92-1.02)$ & 0.21 \\
Weight before botulinum toxin & $1.02(0.96-1.08)$ & 0.61 \\
BMI before botulinum toxin & $1.13(0.94-1.35)$ & 0.21 \\
\hline
\end{tabular}

BMI: body mass index. * Objective treatment outcome: maintenance of baseline weight or weight gain after botulinum toxin treatment 


\section{Follow-up and additional treatment procedures}

Repetitive botulinum toxin injections were performed in 13 patients (varying between 2-10 repeat treatments). Mostly, botulinum toxin treatment was repeated only in case the first treatment was successful. Four gastroparesis patients received a second treatment while the first treatment was not considered to be effective. Other treatment entities patients received are listed in Table 7.5. Mean follow-up was 40 months.

Table 7.5 Additional treatment after botulinum toxin therapy.

\begin{tabular}{lc}
\hline Additional treatment & $\mathbf{N}(\%)$ (Total $\mathbf{n}=\mathbf{4 0})$ \\
\hline Symptoms improved, no further treatment & $11(27.5)$ \\
Diet/prokinetics & $10(25.0)$ \\
Gastric rest and nasoduodenal tube & $6(15.0)$ \\
PEG-J & $10(25.0)$ \\
Laparoscopic jejunostomy & $1(2.5)$ \\
Unknown & $2(5.0)$ \\
\hline
\end{tabular}

PEG-J: Percutaneous Endoscopic Gastrostomy with jejunal extension

\section{Discussion}

In this retrospective analysis, we evaluated single-center data on the clinical effect of endoscopic botulinum toxin injection in patients with decompensated gastroparesis. With respect to symptoms, improvement was reported by $35 \%$ of patients. A substantial group of patients were in need for additional nutritional interventions at long-term follow-up. We specifically sought for predictors of treatment outcome in order to be able to select subgroups of patients with beneficial effect of botulinum toxin treatment. It appeared that neither scintigraphic nor manometric or clinical variables were helpful in predicting treatment outcome.

The symptomatic response rate of $35 \%$ we observed is comparable to previously described retrospective analyses (43-51\%). ${ }^{19,20}$ In addition, a small randomized placebo-controlled trial described symptom improvement in $37 \%$ of patients after botulinum toxin treatment whereas $56 \%$ patients reported symptom improvement after placebo. ${ }^{17}$ Therefore, we have to take into account that the symptom improvement of $35 \%$ in the present study is affected by a placebo response. In addition, $35 \%$ of patients reported weight loss after a single botulinum toxin injection and $42 \%$ of patients eventually needed additional nutritional interventions. Interestingly, pre-treatment BMI was lower in symptom non-responders compared to symptom-responders, which could indicate more severe disease and/or reflect the 
importance of other pathophysiological factors. As treatment with botulinum toxin is expensive and has only a transient effect with need for repeated injections, identification of a subgroup of patients who will benefit from treatment with botulinum toxin is an important objective.

In the present study, we therefore attempted to identify predictors for both subjective and objective treatment outcomes. Several studies, including one retrospective analysis of 179 gastroparesis patients, investigated predictors for symptom improvement and found gender (with inconsistent results reporting either benefit for women or men), lower age, idiopathic gastroparesis and dose of botulinum toxin to be predictors for symptom improvement. ${ }^{19,20}$ We were not able to confirm these results: in our analysis none of the clinical demographic variables was able to predict treatment outcome. However, the smaller sample size in our study compared to that of Coleski et al. may have affected our results. ${ }^{17}$

Apart from clinical predictors for treatment outcome, we hypothesized that more severe delayed gastric emptying and/or antral hypomotility would be associated with a less favorable treatment outcome with both persistent symptoms and inability to maintain weight. However, neither scintigraphic nor manometric parameters were able to predict either objective or subjective treatment outcome after botulinum toxin injection. In line with these results, Friedenburg et al. found no difference in gastric retention between patients with or without symptom improvement after botulinum toxin. ${ }^{17}$ In addition, previous studies were not able to find a correlation between symptoms of gastroparesis and objective gastric emptying measured by scintigraphy. ${ }^{33-37}$ One potential explanation for this lack of correlation is the fact that additional pathophysiological mechanisms apart from delayed gastric emptying (e.g. impaired gastric accommodation, visceral hypersensitivity, psychological factors and comorbidity) contribute to symptom generation and perception. ${ }^{37}$ These factors were not systematically assessed in the current study. Of note is that several prokinetic agents that are generally used for treatment of gastroparesis can also improve symptoms by antiemetic properties or by enhancing gastric accommodation. ${ }^{37}$

Antral hypomotility has been described in a subgroup of gastroparesis patients. ${ }^{38}$ In previous studies, several definitions have been used to describe antral hypomotility (i.e. postprandial motility index (MI) $<13.67 \mathrm{mmHg}$, antral contraction frequency $<1 /$ min or antral contraction frequency $<2 / \mathrm{min}) .{ }^{32,39,40}$ We hypothesized that botulinum treatment in patients with demonstrated antral hypomotility would be less beneficial compared to patients with retained antral motility. However, neither antral contraction frequency nor postprandial antral motility index were able to predict subjective and objective treatment outcome.

Pylorospasm, defined as the presence of episodes of unusually prolonged and intense tonic contractions of the pylorus, has been observed in $42-100 \%$ of patients with diabetic gastroparesis and was hypothesized to be an important target for endoscopic therapy of gastroparesis as well as a potential predictor for treatment 
outcome. $^{7,12,16,41,42}$ Lacy et al. showed a reduction in pylorospasm in a group of diabetic gastroparesis patients treated with botulinum toxin. ${ }^{16}$ In addition, Gupta et al. described a case report of a diabetic gastroparesis patient which showed marked reduction in isolated pyloric pressure waves (IPPWs) after botulinum toxin treatment. ${ }^{43-46}$ Based on these observations, we were interested in analysing pyloric tone and IPPWs in our cohort. Unfortunately, reliable assessment of pyloric tone by high-resolution solid state antroduodenal manometry was not feasible for two main reasons. First, catheter migration occurred frequently which prevents accurate assessment of pyloric pressure especially as the pyloric width is considered to be only $0.6-2.1 \mathrm{~cm}$ and pressure transducers are located at $1 \mathrm{~cm}$ intervals. ${ }^{27,47}$ Despite the use of frequent fluoroscopic examinations and metal clips to identify the pyloric region, catheter migration has also been described by Desipio et al. and it is considered one of the main concerns in HRM antroduodenal manometry. ${ }^{27}$ Second, we were not able to reliably identify a high-pressure zone during phase I episodes, indicative for the pylorus, as previously described by two studies in healthy volunteers. ${ }^{27}$ Consequently, we used the following characteristics to localize the pyloric region: (1) pressure tracing located between antral and small bowel pressure tracings during phase III, (2) fluoroscopic correlation to individual pressure transducers, and (3) manometric morphology representing a hybrid of antral and duodenal morphology. Therefore, we are not able to provide accurate results of pyloric pressure. In our opinion, currently available methodology does not allow prolonged reliable and reproducible recordings of the pyloric pressure zone. We were not able to support the statement of Herbella et al. that identification of the pylorus on HRM is easy and reliable. ${ }^{28}$

Recently evidence was found for impaired pyloric function in patients with gastroparesis using another technique, Endoscopic Functional Luminal Imaging Probe (EndoFLIP). Two previous studies described decreased fasting pyloric compliance in gastroparesis patients compared to healthy controls, and widely varying results with regard to pyloric pressure, distensibility and cross-sectional area in gastroparesis patients. In addition, hydraulic dilatation of the pylorus increased compliance, accelerated gastric emptying and improved quality of life. ${ }^{8,47}$ The hypothesis of an impaired pyloric function as factor in gastroparesis is also supported by Wellington et al. who found evidence for a subgroup of gastroparesis patients with a functional obstructive phenotype with normal gastric pacemaker rhythm. Treatment with either endoscopic botulinum toxin injection or balloon dilation in this group gastroparesis patients resulted in symptom improvement in $75 \%$ of patients. ${ }^{48}$ These results support the hypothesis that improvement of pyloric compliance and/or cross-sectional area by endoscopic treatment options are able to improve symptoms. ${ }^{42}$ Therefore, in the near future the EndoFLIP technique may help to identify a subgroup of gastroparesis patients with low pyloric distensibility and/or small diameter which might benefit from endoscopic botulinum toxin injection or other interventions aimed at reducing pyloric pressure such as endoscopic myotomy (G-POEM). In fact a positive clinical 
response to botulinum toxin injection could also serve to predict the response to GPOEM but this issue remains to be elucidated.

Several limitations of our study should be mentioned. First, this study was retrospective in design and a Global Physician Assessment Scale was used to assess subjective treatment outcome instead of a validated patient-reported outcome measure. Consequently, individual symptoms were not assessed separately. In order to improve the accuracy of the GPA score, two researchers independently assessed GPA-scores with a high concordance rate. Second, several diagnostic modalities were used for measurement of gastric emptying whereas 4 hour scintigraphy is considered the gold standard. Consequently, patients were diagnosed with gastroparesis in case of either increased 4 hour gastric retention or prolonged gastric half emptying time. However, no association was found between the diagnostic method for assessment of gastric emptying (4 hour scintigraphy vs. short scintigraphy or breath test) or inclusion criterion (increased 4 hour gastric retention or prolonged gastric half emptying time), and either subjective or objective treatment outcome. Third, the 4 hour gastric emptying scintigraphy performed at our medical center differs with regard to meal content, and consequently reference values, from the consensus recommendation. For future studies, use of the recommended gastric emptying scintigraphy is preferred although recent data described that only $3.1 \%$ of laboratories were compliant with the recommended protocol. Fourth, high-resolution antroduodenal manometry did not allow us to reliable assess pyloric pressure and pylorospasm.

In conclusion, symptom improvement is achieved in a subgroup of gastroparesis patients treated with endoscopic pyloric botulinum toxin. Although the majority of patients was able to maintain their baseline weight at short-term follow-up, a substantial group of patients needed nutritional interventions on long-term follow-up. Moreover, none of the demographic clinical, scintigraphic or antroduodenal manometric variables were able to predict either subjective or objective treatment outcome. We were therefore unable to provide substantiation for the use of botulinum toxin injections in gastroparesis. 


\section{References}

1. Shin AS, Camilleri M. Diagnostic assessment of diabetic gastroparesis. Diabetes. 2013;62(8):2667-73.

2. Camilleri M, Shin AS. Diagnostic assessment of diabetic gastroparesis. Diabetes. 2013;62:2667-73.

3. Abell TL, Bernstein RK, Cutts T, Farrugia G, Forster J, Hasler WL, et al. Treatment of gastroparesis: a multidisciplinary clinical review. Neurogastroenterol Motil. 2006;18(4):263-83.

4. Ali T, Hasan M, Hamadani M, Harty RF. Gastroparesis. Southern Medical Journal. 2007;100(3):281-6.

5. Parkman HP, Hasler WL, Fisher RS. American Gastroenterological Association technical review on the diagnosis and treatment of gastroparesis. Gastroenterology. 2004;127(5):1592-622.

6. Camilleri M, Brown ML, Malagelada JR. Relationship between impaired gastric emptying and abnormal gastrointestinal motility. Gastroenterology. 1986;91(1):94-9.

7. Mearin F, Camilleri M, Malagelada JR. Pyloric dysfunction in diabetics with recurrent nausea and vomiting. Gastroenterology. 1986;90(6):1919-25.

8. Gourcerol G, Tissier F, Melchior C, Touchais JY, Huet E, Prevost G, et al. Impaired fasting pyloric compliance in gastroparesis and the therapeutic response to pyloric dilatation. Aliment Pharmacol Ther. 2015;41(4):360-7.

9. Langworthy J, Parkman HP, Schey R. Emerging strategies for the treatment of gastroparesis. Expert Rev Gastroenterol Hepatol. 2016;10(7):817-25.

10. Vanheel H, Vicario M, Vanuytsel T, Van Oudenhove L, Martinez C, Keita AV, et al. Impaired duodenal mucosal integrity and low-grade inflammation in functional dyspepsia. Gut. 2014;63(2):262-71.

11. Olausson EA, Storsrud S, Grundin H, Isaksson M, Attvall S, Simren M. A small particle size diet reduces upper gastrointestinal symptoms in patients with diabetic gastroparesis: a randomized controlled trial. Am J Gastroenterol. 2014;109(3):375-85.

12. Camilleri M, Parkman HP, Shafi MA, Abell TL, Gerson L, American College of G. Clinical guideline: management of gastroparesis. Am J Gastroenterol. 2013;108(1):18-37; quiz 8.

13. Ahuja NK, Clarke JO. Pyloric Therapies for Gastroparesis. Curr Treat Options Gastroenterol. 2017; 15(1):230-40.

14. Lacy BE, Weiser K, Kennedy A. Botulinum toxin and gastrointestinal tract disorders: panacea, placebo, or pathway to the future? Gastroenterol Hepatol (N Y). 2008;4(4):283-95.

15. James AN, Ryan JP, Parkman HP. Inhibitory effects of botulinum toxin on pyloric and antral smooth muscle. Am J Physiol Gastrointest Liver Physiol. 2003;285(2):G291-7.

16. Lacy BE, Crowell MD, Schettler-Duncan A, Mathis C, Pasricha PJ. The treatment of diabetic gastroparesis with botulinum toxin injection of the pylorus. Diabetes Care. 2004;27(10):2341-7.

17. Friedenberg FK, Palit A, Parkman HP, Hanlon A, Nelson DB. Botulinum toxin A for the treatment of delayed gastric emptying. Am J Gastroenterol. 2008;103(2):416-23.

18. Arts J, Holvoet L, Caenepeel P, Bisschops R, Sifrim D, Verbeke K, et al. Clinical trial: a randomizedcontrolled crossover study of intrapyloric injection of botulinum toxin in gastroparesis. Aliment Pharmacol Ther. 2007;26(9):1251-8.

19. Coleski R, Anderson MA, Hasler WL. Factors associated with symptom response to pyloric injection of botulinum toxin in a large series of gastroparesis patients. Dig Dis Sci. 2009;54(12):2634-42.

20. Bromer MQ, Friedenberg F, Miller LS, Fisher RS, Swartz K, Parkman HP. Endoscopic pyloric injection of botulinum toxin A for the treatment of refractory gastroparesis. Gastrointest Endosc. 2005;61(7): 833-9.

21. Parkman HP, Fass R, Foxx-Orenstein AE. Treatment of patients with diabetic gastroparesis. Gastroenterol Hepatol. 2010;6(6):1-16.

22. Hasler WL. Gastroparesis--current concepts and considerations. Medscape J Med. 2008;10(1):16.

23. Abell TL, Camilleri M, Donohoe K, Hasler WL, Lin HC, Maurer AH, et al. Consensus recommendations for gastric emptying scintigraphy: a joint report of the American Neurogastroenterology and Motility Society and the Society of Nuclear Medicine. Am J Gastroenterol. 2008;103(3):753-63.

24. Szarka LA, Camilleri M. Methods for measurement of gastric motility. Am J Physiol Gastrointest Liver Physiol. 2009;296(3):G461-75. 
25. Hanauer S, Schwartz J, Robinson M, Roufail W, Arora S, Cello J, et al. Mesalamine capsules for treatment of active ulcerative colitis: results of a controlled trial. Pentasa Study Group. Am J Gastroenterol. 1993;88(8):1188-97.

26. Strijbos D, Keszthelyi D, Masclee AAM, Gilissen LPL. Percutaneous endoscopic colostomy for adults with chronic constipation: Retrospective case series of 12 patients. Neurogastroenterol Motil. 2018; 30(5):e13270.

27. Desipio J, Friedenberg FK, Korimilli A, Richter JE, Parkman HP, Fisher RS. High-resolution solid-state manometry of the antropyloroduodenal region. Neurogastroenterol Motil. 2007;19(3):188-95.

28. Herbella FA, Aprile LR, Patti MG. High-resolution manometry for the evaluation of gastric motility. Updates Surg. 2014;66(3):177-81.

29. Bortolotti M, Annese V, Coccia G. Twenty-four hour ambulatory antroduodenal manometry in normal subjects (co-operative study). Neurogastroenterol Motil. 2000;12(3):231-8.

30. Quigley EM, Donovan JP, Lane MJ, Gallagher TF. Antroduodenal manometry. Usefulness and limitations as an outpatient study. Dig Dis Sci. 1992;37(1):20-8.

31. Verhagen MA, Samsom M, Smout AJ. Gastric myoelectrical and antroduodenal motor activity in patients with achalasia. Neurogastroenterol Motil. 1998;10(3):211-8.

32. Thumshirn M, Bruninga K, Camilleri M. Simplifying the evaluation of postprandial antral motor function in patients with suspected gastroparesis. Am J Gastroenterol. 1997;92(9):1496-500.

33. de Caestecker JS, Ewing DJ, Tothill P, Clarke BF, Heading RC. Evaluation of oral cisapride and metoclopramide in diabetic autonomic neuropathy: an eight-week double-blind crossover study. Aliment Pharmacol Ther. 1989;3(1):69-81.

34. Horowitz M, Harding PE, Maddox AF, Wishart JM, Akkermans LM, Chatterton BE, et al. Gastric and oesophageal emptying in patients with type 2 (non-insulin-dependent) diabetes mellitus. Diabetologia. 1989;32(3):151-9.

35. Keshavarzian A, Iber FL, Vaeth J. Gastric emptying in patients with insulin-requiring diabetes mellitus. Am J Gastroenterol. 1987;82(1):29-35.

36. Loo FD, Palmer DW, Soergel KH, Kalbfleisch JH, Wood CM. Gastric emptying in patients with diabetes mellitus. Gastroenterology. 1984;86(3):485-94.

37. Janssen $\mathrm{P}$, Harris MS, Jones $\mathrm{M}$, Masaoka $\mathrm{T}$, Farre $\mathrm{R}$, Tornblom $\mathrm{H}$, et al. The relation between symptom improvement and gastric emptying in the treatment of diabetic and idiopathic gastroparesis. Am J Gastroenterol. 2013;108(9):1382-91.

38. Thazhath SS, Jones KL, Horowitz M, Rayner CK. Diabetic gastroparesis: recent insights into pathophysiology and implications for management. Expert Rev Gastroenterol Hepatol. 2013;7(2): 127-39.

39. Camilleri M, Hasler WL, Parkman HP, Quigley EM, Soffer E. Measurement of gastrointestinal motility in the GI laboratory. Gastroenterology. 1998;115(3):747-62.

40. Parthasarathy G, Ravi K, Camilleri M, Andrews C, Szarka LA, Low PA, et al. Effect of neostigmine on gastroduodenal motility in patients with suspected gastrointestinal motility disorders. Neurogastroenterol Motil. 2015;27(12):1736-46.

41. Lacy BE, Zayat EN, Crowell MD, Schuster MM. Botulinum toxin for the treatment of gastroparesis: a preliminary report. Am J Gastroenterol. 2002;97(6):1548-52.

42. Snape WJ, Lin MS, Agarwal N, Shaw RE. Evaluation of the pylorus with concurrent intraluminal pressure and EndoFLIP in patients with nausea and vomiting. Neurogastroenterol Motil. 2016;28(5): 758-64.

43. Gupta P, Rao SS. Attenuation of isolated pyloric pressure waves in gastroparesis in response to botulinum toxin injection: a case report. Gastrointest Endosc. 2002;56(5):770-2.

44. Deane AM, Besanko LK, Burgstad CM, Chapman MJ, Horowitz M, Fraser RJ. Modulation of individual components of gastric motor response to duodenal glucose. World J Gastroenterol. 2013;19(35): 5863-9.

45. Fraser RJ, Horowitz M, Maddox AF, Dent J. Postprandial antropyloroduodenal motility and gastric emptying in gastroparesis--effects of cisapride. Gut. 1994;35(2):172-8.

46. Heddle R, Dent J, Toouli J, Read NW. Topography and measurement of pyloric pressure waves and tone in humans. Am J Physiol. 1988;255(4 Pt 1):G490-7. 
47. Malik Z, Sankineni A, Parkman HP. Assessing pyloric sphincter pathophysiology using EndoFLIP in patients with gastroparesis. Neurogastroenterol Motil. 2015;27(4):524-31.

48. Wellington J, Scott B, Kundu S, Stuart P, Koch KL. Effect of endoscopic pyloric therapies for patients with nausea and vomiting and functional obstructive gastroparesis. Auton Neurosci. 2017;202:56-61. 


\section{Chapter 8}

General discussion 



\section{General discussion}

\section{Part I Patient-reported outcome measures in functional dyspepsia}

Functional dyspepsia is a symptom-based diagnosis according to the Rome IV criteria. ${ }^{1}$ Due to the lack of biological markers or objective outcome parameters, use of patientreported outcome measures (PROMs) is recommended to assess treatment efficacy as symptom reduction is considered the primary aim in FD therapy.

In chapter $\mathbf{2}$ we have described psychometric validation properties, strengths and limitations of 20 available, published, outcome measures. Fourteen unidimensional and six multidimensional outcome measures were found with widely varying psychometric validation properties. Currently, none of these outcome measures fulfills all validation criteria defined by regulatory authorities. In fact, several assumptions are not met: (a) lack of assessment of all FD core symptoms which is considered essential to evaluate treatment efficacy. ${ }^{2-7}$ (b) Use of patient-administered instead of investigator-administered PROMs is recommended to reduce social desirability bias and prevent underestimation of symptoms by clinicians. ${ }^{8,9}$ (c) The majority of the questionnaires have not been tested and validated in the target population (i.e. functional dyspepsia patients according to Rome criteria), and (d) the recall period should be $\leq 24$ hours as all questionnaires are based on autobiographical memory and are therefore prone to recall bias. ${ }^{6}$ Additional limitations of retrospective paper questionnaires are ecological bias and non-compliance. These limitations together with the lack of one universal accepted PROM underline the need for development of a novel PROM. ${ }^{10,11}$

Development of a PROM based on the Experience Sampling Method (ESM) seems attractive in order to overcome several limitations of retrospective questionnaires. Most experience with the ESM is currently available for patients with psychiatric disorders. Recently, Verhagen et al. used the ESM-application as mobile Routine Outcome Monitoring (mROM) in 64 patients with psychiatric complaints in an ambulatory mental health care setting. Adequate reliability and validity were described for both positive and negative affect, thereby confirming the applicability of this novel technique as outcome measure in an ambulatory clinical setting. ${ }^{12}$ In this setting, momentary assessment technology is able to evaluate the effect of (antidepressant) medication and detect relapse in case of medication cessation. ${ }^{13,14}$

Besides use of the ESM as an outcome measure to evaluate treatment efficacy, it has the potential to support personalized health care as detailed feedback to patients might enhance insight and facilitate self-management, shared decision making and compliance. ${ }^{15,16}$ In a recent randomized controlled trial, patients with a depressive 
disorder were randomized into a control group which received standard antidepressant medication, whereas participants in the experimental group participated in an ESM procedure and received weekly personalized feedback on patterns of positive affect. Actual levels of positive affect in the context of daily life activities, events and social activities, changes in positive affect, and the number of depressive complaints over the course of the ESM intervention were discussed. Participants in the experimental group demonstrated a greater decline in depressive symptoms over a 32-week period compared to the control group. Moreover, participants appreciated the personalized feedback and tried to apply the suggestions to their daily lives. Therefore, use of add-on ESM may be effective as therapeutic tool to complement standard medical therapy. ${ }^{17}$

In addition to the positive therapeutic effect of personalized feedback, the ESM principle can also be used to implement psychotherapy in daily lives. In a recent clinical trial, acceptance and commitment therapy was applied to patients with depressive disorders using ESM. Therapy consisted of both regular exercises independent of current thoughts, feelings or situations, and ACTion exercises which are applicable in distressing situations. Adherence to this ESM-based training was high and appeared to be a feasible intervention, although the long-term effects still need to be evaluated. ${ }^{18}$

Despite the increasing use of ESM in patients with psychiatric disorders, its use in patients with gastrointestinal disorders is currently limited to two small trials in patients with Irritable Bowel Syndrome (IBS). Weinland et al. used momentary assessment in 77 IBS patients and evaluated pain, bloating, stress and stool consistency according to the Bristol stool form scale. ${ }^{19}$ Momentary assessment scores were compared with end-of-day diary scores. Over-reporting of pain on end-of-day diaries was found, and end-of-day scores tracked most closely the maximum score on the digital momentary assessment. Comparable results were described by Mujagic et al. $^{20}$ Whereas Weinland et al. assessed only four symptoms, Mujagic et al. used a 60 -item questionnaire with assessment of gastrointestinal symptoms, physical and mental experiences, and activities and contextual aspects. This questionnaire was based on previously used outcome measures for assessment of IBS symptoms and ESM-questionnaires used in psychiatric studies, and therefore did not meet the assumption for PROM development according to a conceptual framework with modification of this framework by input from patient focus groups. In order to meet this assumption Vork et al. performed patient focus groups interviews, an international expert meeting and cognitive interviews and developed a 32-item ESMbased PROM for assessment of IBS symptoms. This novel PROM will be validated in IBS patients in The Netherlands, Belgium, United Kingdom and Sweden. ${ }^{21}$

In line with the study of Vork et al., we describe the first step in the development of an ESM-based PROM for symptom assessment in patients with functional dyspepsia (chapter 3). Based on the FDA recommendations, we developed a conceptual 
framework and acquired input from patients by organizing patient focus groups. Subsequently, an international expert meeting was organized and cognitive interviews were performed to assure on patients' understanding. This led to the development of a 33-item ESM-based PROM with adequate content validity.

Next step in the development of this novel PROM is to perform additional psychometric validation in dyspeptic patients with assessment of reliability, validity and responsiveness. ${ }^{6}$ Evaluation of internal consistency and test-retest reliability is necessary to demonstrate adequate reliability. Moreover, the ESM-based PROM should be compared with existing FD outcome measures (e.g. Nepean Dyspepsia Index) and psychiatric questionnaires (e.g. Hospital Anxiety and Depression Scale) to confirm construct validity, whereas 'known-groups' validity can be assessed by comparing scores between FD patients and healthy controls. In addition, the novel PROM should be used in a treatment trial to evaluate the ability to detect change. ${ }^{22}$ During the additional development of this outcome measure, it is moreover important to evaluate whether a FDA approved endpoint can be defined.

Potential advantages of the novel ESM-based PROM compared to conventional methods are: (a) repeated assessment of current symptoms limiting both recall bias and over-reporting of symptoms, (b) improvement of ecological validity by symptom assessment in patients' daily clinical life, (c) promoting compliance by use of a digital sampling method instead of paper questionnaires, and (d) acquire information about symptom triggers with the potential for extensive feedback and personalized treatment. Therefore, we hypothesize that this novel ESM-based PROM has the potential to become an excellent outcome measure. Future studies with ESM in dyspepsia should focus on the evaluation of psychometric validation properties.

\section{Part II Prediction of treatment outcome in upper gastrointestinal disorders}

Treatment options for patients with upper gastrointestinal disorders can be roughly divided into (a) medical therapy, (b) endoscopic interventions and (c) surgical procedures. First line therapy for patients with GERD consists of acid suppressive medication, whereas patients with gastroparesis are treated with prokinetic agents in addition to lifestyle advice. In case these medical options are ineffective or cause substantial side effects, endoscopic or surgical interventions will be considered. In the last decades, a shift has been observed from invasive surgical interventions to minimally invasive (endoscopic) treatment modalities in order to reduce (per)operative risks and prevent development of (long-term) complications. ${ }^{23-25}$ (Longterm) complications of traditional antireflux surgery are postoperative dysphagia, gasbloating syndrome and gastroparesis due to vagal nerve injury, whereas laparoscopic 
Heller myotomy in achalasia patients is followed by a fundoplication to prevent postoperative reflux disease. Common feature of minimally invasive (endoscopic) treatment options appears to be the limited efficacy for subgroups of patients. ${ }^{24-27}$ Adequate patient selection by the use of diagnostic modalities is therefore warranted to optimize treatment outcome and reduce health care costs.

Esophagogastric junction (EGJ) distensibility is one potential parameter of interest for selection of patients for minimally invasive (endoscopic) interventions. EGJ distensibility was first assessed with a barostat device which was however cumbersome and required additional fluoroscopic evaluation. In order to overcome these limitations, a Functional Luminal Imaging Probe (EndoFLIP) was developed.

In the last decade, several trials have been performed with this novel EndoFLIP technique in patients with various upper gastrointestinal disorders including GERD, achalasia, gastroparesis and eosinophilic esophagitis. The EndoFLIP has been used in order (a) to acquire information about pathophysiological mechanisms in upper gastrointestinal disorders, (b) to evaluate the effect of several endoscopic and surgical treatment modalities, (c) to identify subgroups of patients with potential beneficial treatment outcome, and (d) to guide gastroenterologists and/or surgeons during endoscopic and surgical interventions.

\section{Achalasia}

The EndoFLIP has been used in several trials and decreased EGJ distensibility has been reported in newly diagnosed achalasia patients compared to healthy volunteers. ${ }^{28-32}$ (chapter 4) In addition, a subgroup of patients with clinical and radiological features of achalasia but with normal EGJ relaxation on HRM demonstrated decreased EGJ distensibility and favorable response on achalasia treatment. These patients are therefore considered a subgroup of achalasia patients in which the EndoFLIP technique has additional diagnostic value. ${ }^{29}$

Recently, FLIP topography was used to assess esophageal body contractility in achalasia subtypes. ${ }^{31}$ Absent peristalsis was common in type I patients (68-73\%), type II patients demonstrated contractility in $65 \%$ whereas the majority of type III patients had repetitive retrograde contractions. Therefore, FLIP topography can be used to detect esophageal contractility which has not been observed with manometry and contribute to a better understanding of pathophysiological mechanisms of achalasia subtypes and potentially predict achalasia disease course and treatment efficacy.

Achalasia treatment is focused on improving symptoms by decreasing the EGJ outflow obstruction by either pneumatic balloon dilation (PD), (laparoscopic) Heller myotomy with fundoplication (LHM), or peroral endoscopic myotomy (POEM). Declining success rates after achalasia treatment lead to retreatment in a substantial subgroup of patients. Identification of patients in need of retreatment is however challenging, as symptoms are underreported or absent due to impaired esophageal 
sensitivity and/or esophageal decompensation. ${ }^{28}$ Consequently, symptoms are not reliable to determine therapeutic strategy in achalasia patients, and the optimal test for evaluation of treatment outcome is still a matter of debate. ${ }^{28,33}$ Esophageal manometry has been repeatedly proposed as a useful test, as several studies have shown that post-treatment LES pressure $<10 \mathrm{mmHg}$ was highly predictive of beneficial clinical outcome. ${ }^{34-36}$ However, a significant proportion of patients with persistent symptoms have a LES pressure $<10 \mathrm{mmHg}$ with a lack of correlation between clinical outcome and LES pressure. ${ }^{28,33,37}$ In addition to manometry, esophageal emptying assessed with a Timed Barium Esophagogram (TBE) has been proposed as diagnostic test to predict clinical outcome. Concordance between symptom reduction and improved esophageal emptying has been found in $52-89 \%$ of patients. ${ }^{28,37-39}$ Due to the lack of concordance in the remaining patients together with the weak correlation between both variables, Vaezi et al. recommended the use of TBE in all patients after treatment to verify adequate esophageal emptying. ${ }^{37-39}$

The decreased EGJ distensibility in achalasia patients, assessed with the EndoFLIP device, led to the hypothesis that EGJ distensibility could potentially be a useful parameter to evaluate and predict treatment outcome. Previous studies described higher EGJ distensibility in patients with successful treatment outcome compared to both untreated achalasia patients and patients with a poor treatment response. ${ }^{28,32}$ In chapter $\mathbf{4}$ we evaluated the effect of PD in newly diagnosed achalasia patients and we found a direct post-PD increase in EGJ distensibility. ${ }^{30}$ An increased EGJ distensibility has also been described three months after PD, and several studies described an improved EGJ distensibility after POEM and LHM. ${ }^{28,40-47}$ More important is however the relation between (the improvement in) EGJ distensibility and clinical outcome. Several studies reported higher EGJ distensibility and EGJ diameter in patients with good clinical response compared to patients with persistent symptoms, and the majority of patients reached distensibility values above the cut off value for normality after treatment with either PD or POEM. ${ }^{28,30,41,43}$ However, in chapter $\mathbf{4}$ we described that post-PD distensibility is not able to discriminate between responders and nonresponders, as five out of six non-responders reached distensibility values above the cut off value for normality after PD. Moreover, no correlation could be found between post-PD EGJ distensibility and clinical outcome at both one month and one year follow-up (chapter 4). In line with our results, a recent study confirmed the lack of a correlation between post-PD EGJ distensibility and short-term clinical response. ${ }^{48}$ In contrast, Rohof et al. found a correlation between the change in EGJ distensibility and clinical response, and considered EGJ distensibility an appropriate parameter to evaluate treatment outcome. ${ }^{28}$ Potential explanations for the varying results are the fact that we measured EGJ distensibility direct after PD which might give an underestimation of EGJ distensibility due to post-procedural edema, graded PD might potentially lead to an additional increase in distensibility, the use of varying dilation protocols and a difference in distribution of achalasia subtypes between trials. 
Additional research is necessary, but the EndoFLIP may still prove to be useful in identifying patients with high risk of relapse after treatment with consequent intensive monitoring of these patients and schedule additional treatment procedures in order to prevent development of esophageal decompensation and esophageal carcinoma.

In addition to EGJ distensibility, two novel parameters in need for future research to evaluate the role in het management of achalasia patients are the 'bolus flow time', assessed with high-resolution impedance manometry, and 'esophageal pressurization', assessed with the rapid drinking test during high-resolution manometry. ${ }^{49,50}$ Carlson et al. demonstrated that bolus flow time could better discriminate achalasia patients by clinical outcomes compared with established HRM metrics of EGJ pressure and integrated relaxation pressure (IRP). ${ }^{50}$ This might be due to the fact that bolus flow time incorporates measures of both EGJ opening and flowpermissive pressure gradient required for flow across the EGJ, which provides a more comprehensive evaluation of esophageal function compared to EGJ pressure and IRP..$^{50}$ In addition, the rapid drinking test is a novel method to measure esophageal stasis. Achalasia patients demonstrate higher EGJ pressures and increased esophageal body pressurization during the rapid drinking test compared to healthy controls. ${ }^{49}$ Esophageal body pressurization during the rapid drinking test is correlated with esophageal stasis assessed with TBE, and additional research is necessary to evaluate whether this rapid drinking test can predict long-term clinical treatment outcome.

Apart from the potential role of the EndoFLIP to select patients with high risk of relapse after treatment, this technique can be used intra-operatively to guide LHM or POEM. Many surgeons choose to assess the adequacy of LHM intraoperatively using visual anatomic landmarks or endoscopy, and a subgroup of patients will achieve inadequate control of achalasia symptoms due to insufficient myotomy. Intraoperative use of the EndoFLIP revealed an increase in EGJ distensibility and CSA after LHM with a partial decrease in EGJ distensibility after fundoplication. ${ }^{46,51}$ The EndoFLIP might therefore guide surgeons and gastroenterologists during LHM and POEM, which might lead to personalized care in order to improve clinical outcome and prevent development of (long-term) complications. Current limitation is the fact that no optimal EGJ diameter or EGJ distensibility is available as treatment target. Based on a clinical trial in achalasia patients treated with either LHM or POEM, a target EGJ distensibility between $4.5-8.5 \mathrm{~mm}^{2} / \mathrm{mmHg}$ has been supposed to prevent both persistent symptoms and development of GERD. ${ }^{45}$ However, additional research is necessary to confirm this target EGJ distensibility.

The Esophageal Functional Luminal Imaging Probe (EsoFLIP) is an interesting novel device developed by the manufacturers of the EndoFLIP. The EsoFLIP is a $30-\mathrm{mm}$ hydraulic dilation catheter which can be used for (a) assessment of EGJ distensibility and (b) therapeutic dilation of the EGJ. During EGJ dilation, the shape of the balloon is visible on the recording unit thereby obviating the need for fluoroscopy. Ten achalasia 
patients were treated with EsoFLIP in a feasibility study. ${ }^{52}$ All patients underwent two EsoFLIP dilations with a two day interval. Technical success was achieved in all patients and no complications occurred with this novel technique. An increase in EGJ distensibility was observed after treatment and clinical success rate was $70 \%$ at three months follow-up. Recurrent symptoms with need for LHM after treatment with EsoFLIP might be due to the lack of a $35 \mathrm{~mm}$ and $40 \mathrm{~mm}$ balloon, which limits the potential for graded hydraulic dilation.

In conclusion, use of the EndoFLIP in achalasia patients has provided information about pathophysiological mechanisms with regard to EGJ distensibility and esophageal contractility, including differences between achalasia subtypes. In addition, the EndoFLIP has additional diagnostic value in patients with classic symptoms and radiological features but with normal EGJ relaxation on HRM, and can be used to evaluate treatment efficacy, however, findings remain incongruent. One of the most promising applications seems to be its intraoperative use to guide LHM and POEM.

\section{Gastroesophageal reflux disease}

Surgical treatment for GERD is considered in patients with persistent symptoms despite use of acid suppressive medication or in case of side effects. Mechanical failure and inadequate patient selection are two factors associated with treatment failure after surgical fundoplication. In order to improve patient selection, a systematic review has been performed in GERD patients treated with surgical fundoplication to identify predictors of treatment outcome. ${ }^{53}$ Several patient characteristics (i.e. age, sex, body mass index), preoperative esophagitis, and esophageal dysmotility were not associated with treatment outcome. ${ }^{53}$ Moreover, no consistent associations were found between preoperative response to acid suppressive medication, baseline symptoms, esophageal acid exposure time, and degree of LES competence with treatment outcomes. ${ }^{53}$ Difficult is however the large heterogeneity between studies included in this systematic review with (a) differences in disease severity, (b) use of different classification systems for description of symptoms and variations in esophageal $\mathrm{pH}$ testing, (c) multiple subtle variations in surgical techniques, and (d) variety of subjective and objective outcomes with different definitions to describe study endpoints. ${ }^{53}$ Lack of one uniform definition of failure after antireflux surgery makes it difficult to compare results across studies. ${ }^{54}$

Adequate patient selection seems even more important for TIF compared to surgical fundoplication, due to the limited efficacy for a subgroup of GERD patients. Patients with small $(\leq 2 \mathrm{~cm})$ or no hiatal hernia, and Hill grade I/II are considered the best candidates for successful TIF therapy based on the limited ability to restore EGJ competence. ${ }^{55,56}$ In addition, several studies tried to identify predictors of treatment outcome after TIF. The number of fasteners deployed and intact esophageal motility 
were identified as predictors for beneficial treatment outcome based on cessation of acid suppressive medication post-TIF. ${ }^{56,57}$ Moreover, presence of preoperative esophagitis was a predictor of treatment failure with the demand for revisional laparoscopic fundoplication. ${ }^{26}$ However, results are limited and non-conclusive.

Previous studies described a reduction in EGJ distensibility after Nissen fundoplication, measured with either the barostat device or novel EndoFLIP device. ${ }^{58-60}$ Moreover, EGJ distensibility is still decreased five years after antireflux surgery compared to patients without surgical treatment. ${ }^{61}$ Reduction of EGJ distensibility therefore appears an important therapeutic aim and the EndoFLIP technique could offer preoperative insight into reflux mechanisms and potentially be used as tool to predict treatment outcome. However, conflicting results have been described with regard to the difference in EGJ distensibility between healthy controls and GERD patients as assessed with the EndoFLIP technique. ${ }^{62,63}$ Potential explanations for this conflicting results are (a) differences in patient selection, and (b) large variance in distensibility results in both GERD patients and healthy controls. ${ }^{63,64}$

In chapter $\mathbf{5}$ and $\mathbf{6}$ we evaluated the effect of the minimally invasive antireflux intervention TIF on EGJ competence with the EndoFLIP device and found a direct postoperative decrease in EGJ distensibility. ${ }^{65,66}$ However, six months after TIF no change in EGJ distensibility was found compared to baseline values. One potential explanation could be an overestimated postoperative decrease due to the presence of edema. Deterioration of the created fundoplication over time could be another explanation, as a large randomized controlled trial described deterioration of the Hill grade over time. Directly after TIF, $90 \%$ of patients had a Hill grade I which was reduced to $35 \%$ at 12 months follow-up. ${ }^{26}$

Despite the lack of a change in EGJ distensibility six months after TIF, a subgroup of patients achieved normalization of acid exposure time (56\%) with reduction of the number of reflux episodes. No difference in EGJ distensibility was found between patients with normalized acid exposure time and patients with persistent elevated acid exposure. In line with this results, a recent study found no correlation between EGJ distensibility, esophageal acid exposure time and number of reflux episodes. ${ }^{67}$ Therefore we have to consider other mechanisms in contrast to a change in EGJ distensibility as potential antireflux mechanisms and this also questions the added value of the preoperative EGJ distensibility assessment using EndoFLIP as predictor or parameter of response.

Changes in Transient Lower Esophageal Sphincter Relaxations (TLESRs) are a potential explanation as previous studies described a higher proportion of TLESRs associated with acidic reflux in GERD patients compared to controls with a reduction of TLESRs after traditional antireflux surgery. ${ }^{61,68,69}$ In chapter 5 we evaluated the effect of the minimal invasive TIF procedure on TLESRs and found a reduction in both the total number of TLESRs and the number of TLESRs associated with liquidcontaining reflux. ${ }^{65}$ Although we first hypothesized that this effect might be due to a 
decrease in EGJ distensibility, the results in chapter 6 seem to contradict this as no significant change in EGJ distensibility is present at six months follow-up. ${ }^{66}$ Repair of small hiatal hernias and influence of TIF on the acid pocket might be other factors contributing to reflux control after TIF. ${ }^{70,71}$

Instead of developing minimally invasive (endoscopic) treatment options for GERD, modification of the traditional Nissen or Toupet fundoplication might be an alternative option to reduce postoperative dysphagia and gas-bloating syndrome. Intraoperative use of the EndoFLIP seems an attractive option to evaluate whether this technique can be used as a calibration method for hiatal hernia repair and fundoplication. Currently, the most important limitation is the lack of a value for 'normal EGJ distensibility' and a target for 'post-fundoplication EGJ distensibility', at least partially due to the wide range of EGJ distensibility results in healthy controls. ${ }^{64}$ In a recent study, intraoperative EndoFLIP was used to perform hiatal hernia repair and fundoplication in order to reach a postoperative EGJ distensibility of about $1 \mathrm{~mm}^{2} / \mathrm{mmHg}^{72}$ This target EGJ distensibility was based on a previous trial with intraoperative use of the EndoFLIP during Nissen fundoplication in which a postoperative EGJ distensibility of $1.4 \mathrm{~mm} / \mathrm{mmHg}$ was reached. Moreover, in this trial a case was described in which the wrap appeared too tight during surgery and intraoperative use of the EndoFLIP demonstrated an EGJ distensibility of $0.65 \mathrm{~mm}^{2} / \mathrm{mmHg}$ which was considered too low. Therefore, surgeons modified their intervention to a posterior $270^{\circ}$ fundoplication with a postoperative EGJ distensibility of $0.90 \mathrm{~mm}^{2} / \mathrm{mmHg}$. The patient was free of reflux symptoms but long-term clinical outcome was not available. $^{73}$

In conclusion, the most promising application of the EndoFLIP technique in patients with GERD seems to be its intraoperative use to guide calibration of hiatal hernia repair and fundoplication. Additional studies are however necessary to define 'normal values of EGJ distensibility' and develop 'target values' for EGJ distensibility and EGJ diameter after fundoplication in order to achieve adequate symptom control and prevent long-term complications.

\section{Functional dyspepsia}

The pathophysiology of functional dyspepsia (FD) is considered multifactorial and although it is currently incompletely understood, several mechanisms are thought to contribute to symptom generation including impaired gastric accommodation, disturbed gastric emptying, and hypersensitivity to gastric distension. ${ }^{74,75}$ Although the Rome III consensus panel proposed that different pathophysiological mechanisms would contribute to PDS and EPS symptoms, contradictory results have been reported with wide overlap between PDS and EPS subtypes which limits the utility of the FD subtype classification as guide for therapeutic choices in daily clinical practice. ${ }^{74,76}$ 
In line with these results, a new management algorithm recently published by the American College of Gastroenterology (AGA) recommends the consecutive use of acid suppressive medication, antidepressants and prokinetic agents regardless of FD subtype. ${ }^{77}$ There is no role in FD for endoscopic or surgical interventions. Although these medical treatment options together with eradication of Helicobacter Pylori infection might reduce dyspeptic symptoms, the lack of approved treatment options for FD is remarkable with numbers needed to treat between 6 and $12.5 .^{75}$ This raises the question whether prediction of treatment response is possible in order to improve treatment outcome. However, currently two factors limit the prediction of treatment outcome. First, the proposed multifactorial pathophysiology, with lack of one main abnormality, questions which pathophysiological mechanism should be used to predict treatment outcome. Second, assessment of several pathophysiological abnormalities in FD is challenging. For instance, several options have been developed for assessment of gastric accommodation. ${ }^{75,78}$ Assessment of gastric accommodation with the barostat is considered the gold standard, but it is invasive, time consuming, uncomfortable for patients and it might interfere with normal gastric physiology which limits its feasibility in routine clinical practice. Single photon emission computed tomography (SPECT) is able to detect changes in postprandial gastric volumes but it is less suitable for detection of changes in gastric tone, and the radiation burden is a main disadvantage. The nutrient drinking test was designed to assess early satiety which serves as surrogate marker of proximal stomach function, but use of this test with different drinks and varying drinking rates led to contradictory results. A relatively novel method to assess gastric accommodation is the measurement of intragastric pressure (IGP) combined with nutrient drink ingestion. ${ }^{79}$ Nutrient drink ingestion led to a decrease in IGP with a gradual recovery thereafter, which is correlated with an increase in satiation scores. Therefore, assessment of IGP is probably a more physiological and less invasive method to assess gastric accommodation compared to barostat. ${ }^{78,79}$

Currently, two studies tried to predict the response to acid suppressive medication in FD patients. Bolling-Sternevald et al. described that fewer days with symptoms during the first week of PPI treatment (i.e. early response) predicted higher response rates at the end of a 4-week treatment period. ${ }^{80}$ However, Tally et al. described that a 1-week acid suppression trial had only limited utility in defining patients who are likely or unlikely to respond to a longer period of acid suppression. ${ }^{81}$

In conclusion, additional research is necessary to unravel the pathophysiological mechanisms in FD, to develop (minimally invasive) diagnostic methods to assess these pathophysiological abnormalities, and identify predictors of treatment outcome.

\section{Gastroparesis}

Gastroparesis is a multifactorial disorder and impaired pyloric function is considered one of the pathophysiological mechanisms involved. Manometric studies of patients 
with diabetic gastroparesis demonstrated prolonged periods of increased tonic pyloric activity with high amplitude called pylorospasm which might be due to damage of the autonomic neural pathways. ${ }^{82}$ Based on these findings, several treatment options have been developed in order to improve pyloric function including botulinum toxin treatment. Treatment with botulinum toxin reduced pylorospasm in a small trial of patients with diabetic gastroparesis. ${ }^{83}$ However, use of botulinum toxin for treatment of gastropareses is not recommended by the American College of Gastroenterology guideline as two small placebo-controlled randomized trials found no superior symptom improvement compared to placebo. ${ }^{24,25,84}$

In chapter 7 we describe the clinical effect of botulinum toxin and reported symptom improvement in $35 \%$ of patients which is in line with previous trials. ${ }^{25,85,86}$ Selection of patients for botulinum toxin treatment seems therefore warranted to improve treatment outcome and reduce health care costs as botulinum toxin is expensive and requires repeated treatment sessions. Gender, age, etiology of gastroparesis and dose of botulinum toxin were identified as predictors of treatment outcome by previous studies. ${ }^{85,86}$ In an open study we were not able to confirm these results nor could scintigraphic parameters predict clinical outcome (chapter 7). Use of scintigraphic variables to predict clinical treatment outcome in gastroparesis patients treated with botulinum toxin is limited. In line with our results, two previous studies found no evidence for a predictive effect of pre-treatment gastric retention according to scintigraphy on clinical outcome after botulinum toxin treatment. ${ }^{25,85}$ Lack of a correlation between symptom improvement and change in gastric emptying has been described in a large meta-regression analysis, and it is hypothesized that symptoms of gastroparesis may be caused by other mechanisms including visceral hypersensitivity and impaired gastric accommodation. ${ }^{87}$ With regard to antroduodenal manometry, the major limitation of our study was the inability to identify a high-pressure zone indicative for the pylorus with HRM. In a previous study, no difference was found in basal and peak pyloric pressures between (a) patients with nausea/vomiting with normal or slightly delayed gastric emptying, and (b) patients with severely delayed gastric emptying. ${ }^{88}$

Assessment of pyloric distensibility with the EndoFLIP device might be a novel method to acquire information about pyloric function in gastroparesis patients. Pyloric distensibility is reduced in gastroparesis patients compared to both healthy controls and patients with nausea/vomiting but without delayed gastric emptying. ${ }^{88,89}$ According to the results of healthy controls, a cut off value for normality is potentially $>10 \mathrm{~mm} / \mathrm{mmHg} .{ }^{89}$ Pyloric distensibility was below this cut off value in $38 \%$ of gastroparesis patients and together with the remarkable wide range of pyloric distensibility this seems to support the hypothesis that impaired pyloric function is present in only a subgroup of gastroparesis patients. ${ }^{89,90}$ Inverse correlations were found between (1) pyloric distensibility, CSA and diameter, and (2) dyspeptic 
symptoms whereas conflicting results were described with regard to the correlation between pyloric distensibility and gastric emptying. ${ }^{89-91}$

The effect of treatment on pyloric distensibility has currently been described in two small clinical trials. Ten patients with pyloric distensibility below the cut off value for normality underwent hydraulic dilation of the pylorus. Ten days after treatment an increase in pyloric distensibility was described with an accelerated gastric emptying and improved quality of life. ${ }^{89}$ In addition, 20 gastroparesis patients were treated with gastric peroral pyloromyotomy (G-POEM). Three months after G-POEM, an increase in both pyloric distensibility and pyloric diameter was found. A pre-procedure pyloric distensibility threshold of $<9.2 \mathrm{~mm}^{2} / \mathrm{mmHg}$ predicted a beneficial response with both specificity and positive predictive value of $100 \% .{ }^{91}$ Additional research is however necessary to assess the correlation with clinical outcome. Potentially the EndoFLIP could be used to select gastroparesis patients with impaired pyloric distensibility for endoscopic or surgical treatment options in order to improve treatment outcome. Moreover, it could be hypothesized that intraprocedural use of the EndoFLIP during G-POEM might guide the extent of the myotomy to improve clinical outcome and prevent development of complications.

In conclusion, impaired pyloric distensibility seems to be present in a subgroup of patients with gastroparesis. The EndoFLIP could potentially identify these patients in order to improve treatment outcome by selecting the optimal treatment modality.

\section{Future perspectives of the EndoFLIP technique}

Besides use of the EndoFLIP technique in patients with reflux disease, achalasia and gastroparesis, several studies have evaluated both EGJ distensibility and esophageal distensibility in patients with eosinophilic esophagitis (EOE). Patients with EOE have reduced EGJ distensibility and esophageal distensibility compared to healthy controls, and reduced esophageal distensibility is able to predict the risk for food impaction and requirement for esophageal dilation therapy. ${ }^{92,93}$ Development of FLIP topography led to more accurate and detailed information about esophageal functioning in patients with EOE as FLIP topography is able to detect regional variations of disease involvement. In EOE patients, FLIP topography is able to detect focal strictures, narrow caliber esophagus, and globally diminished esophageal distensibility without endoscopic abnormalities. ${ }^{94}$ In the future, use of FLIP topography might detect esophageal strictures either in EOE patients, strictures due to longstanding GERD or after esophageal surgery for subsequent treatment with esophageal dilation therapy. In line with the EsoFLIP balloon for esophageal dilation in achalasia patients, the manufacturer of the EndoFLIP developed a smaller EsoFLIP balloon for treatment of these esophageal strictures. However, the first results of this novel EsoFLIP balloon for treatment of esophageal strictures are still awaited. 
In addition to the selection of patients for esophageal therapies, use of FLIP topography can also provide gastroenterologists with (additional) diagnostic information for patients with non-obstructive dysphagia. In a recent study, 145 patients with non-obstructive dysphagia were evaluated with both highresolution manometry (HRM) and EndoFLIP. Although 34 patients had no major motility disorder on HRM, $50 \%$ of patients had abnormal FLIP topography findings. Additional research is necessary to evaluate the clinical relevance of these findings, but FLIP topography seems to provide a complementary method to HRM for evaluation of dysphagia. ${ }^{95}$

Therefore, EndoFLIP, EsoFLIP and FLIP topography techniques provided information about pathophysiological mechanisms and are potentially able to stratify patients for therapy and evaluate the effect of treatment. However, additional studies are necessary to define 'normal values' and 'target values' to guide different treatment modalities in order to improve clinical outcome and prevent development of complications. In addition, correlation between these objective and quantifiable measurements, whether performed pre- or post-treatment, and patients' symptoms, or better said patient reported outcome measures, need to be firmly established. Methodologies based on the ESM principle, such as described in part I, can also be of added value in this respect. 


\section{References}

1. Stanghellini V, Talley NJ, Chan F, Hasler WL, Malagelada J, Suzuki H, et al. Rome IV - Gastroduodenal Disorders. Gastroenterology. 2016.

2. (FDA) FaDA. Guidance for industry. Patient-reported outcome measures: use in medical product development to support labeling claims. 2009.

3. Carbone F, Vandenberghe A, Holvoet L, Vanuytsel T, Van Oudenhove L, Jones M, et al. Validation of the Leuven Postprandial Distress Scale, a questionnaire for symptom assessment in the functional dyspepsia/postprandial distress syndrome. Aliment Pharmacol Ther. 2016;44(9):989-1001.

4. Leidy NK, Farup C, Rentz AM, Ganoczy D, Koch KL. Patient-based assessment in dyspepsia: development and validation of Dyspepsia Symptom Severity Index (DSSI). Dig Dis Sci. 2000;45(6):1172-9.

5. Talley NJ, Haque M, Wyeth JW, Stace NH, Tytgat GN, Stanghellini V, et al. Development of a new dyspepsia impact scale: the Nepean Dyspepsia Index. Aliment Pharmacol Ther. 1999;13(2):225-35.

6. (FDA) FaDA. Guidance for Industry; Patient-Reported Outcome Measures: use in Medical Product Development to Support Labeling Claims 2009.

7. Taylor F, Higgins S, Carson RT, Eremenco S, Foley C, Lacy BE, et al. Development of a symptomfocused patient-reported outcome measure for functional dyspepsia: The Functional Dyspepsia Symptom Diary (FDSD). Am J Gastroenterol. 2018;113(1):39-48.

8. Moayyedi P, Duffett S, Braunholtz D, Mason S, Richards ID, Dowell AC, et al. The Leeds Dyspepsia Questionnaire: a valid tool for measuring the presence and severity of dyspepsia. Aliment Pharmacol Ther. 1998;12(12):1257-62.

9. el-Omar EM, Banerjee S, Wirz A, McColl KE. The Glasgow Dyspepsia Severity Score--a tool for the global measurement of dyspepsia. Eur J Gastroenterol Hepatol. 1996;8(10):967-71.

10. Shiffman S, Stone AA, Hufford MR. Ecological momentary assessment. Annu Rev Clin Psychol. 2008;4:1-32.

11. Stone AA, Shiffman S, Schwartz JE, Broderick JE, Hufford MR. Patient compliance with paper and electronic diaries. Control Clin Trials. 2003;24(2):182-99.

12. Verhagen SJW, Berben JA, Leue C, Marsman A, Delespaul P, van Os J, et al. Demonstrating the reliability of transdiagnostic mHealth Routine Outcome Monitoring in mental health services using experience sampling technology. PLoS One. 2017;12(10):e0186294.

13. Wichers M, Groot PC, Psychosystems ESMGEWSG. Critical Slowing Down as a Personalized Early Warning Signal for Depression. Psychother Psychosom. 2016;85(2):114-6.

14. Bak M, Drukker M, Hasmi L, van Os J. An $n=1$ Clinical Network Analysis of Symptoms and Treatment in Psychosis. PLoS One. 2016;11(9):e0162811.

15. van Os J, Verhagen S, Marsman A, Peeters F, Bak M, Marcelis M, et al. The experience sampling method as an mHealth tool to support self-monitoring, self-insight, and personalized health care in clinical practice. Depress Anxiety. 2017;34(6):481-93.

16. Verhagen SJ, Hasmi L, Drukker M, van Os J, Delespaul PA. Use of the experience sampling method in the context of clinical trials. Evid Based Ment Health. 2016;19(3):86-9.

17. Kramer I, Simons CJ, Hartmann JA, Menne-Lothmann C, Viechtbauer W, Peeters F, et al. A therapeutic application of the experience sampling method in the treatment of depression: a randomized controlled trial. World Psychiatry. 2014;13(1):68-77.

18. Batink T, Bakker J, Vaessen T, Kasanova Z, Collip D, van Os J, et al. Acceptance and Commitment Therapy in Daily Life Training: A Feasibility Study of an mHealth Intervention. JMIR Mhealth Uhealth. 2016;4(3):e103.

19. Weinland SR, Morris CB, Hu Y, Leserman J, Bangdiwala SI, Drossman DA. Characterization of episodes of irritable bowel syndrome using ecological momentary assessment. Am J Gastroenterol. 2011;106(10):1813-20.

20. Mujagic Z, Leue C, Vork L, Lousberg R, Jonkers DM, Keszthelyi D, et al. The Experience Sampling Method--a new digital tool for momentary symptom assessment in IBS: an exploratory study. Neurogastroenterol Motil. 2015;27(9):1295-302. 
21. Vork L, Keszthelyi D, Mujagic Z, Kruimel JW, Leue C, Ponten I, et al. Development, content validity, and cross-cultural adaptation of a patient-reported outcome measure for real-time symptom assessment in irritable bowel syndrome. Neurogastroenterol Motil. 2018;30(3).

22. US. Department of Health and Human Services, FDA center for Drug Evaluation and Research, et al. Guidance for industry: patient-reported outcome measures: use in medical product development to support labeling claims: draft guidance. Health Qual Life Outcomes. 2006;4:79.

23. Ganz RA. A review of new surgical and endoscopic therapies for gastroesophageal reflux disease. Gastroenterol Hepatol 2016;12(7):424-31.

24. Arts J, Holvoet L, Caenepeel P, Bisschops R, Sifrim D, Verbeke K, et al. Clinical trial: a randomizedcontrolled crossover study of intrapyloric injection of botulinum toxin in gastroparesis. Aliment Pharmacol Ther. 2007;26(9):1251-8.

25. Friedenberg FK, Palit A, Parkman HP, Hanlon A, Nelson DB. Botulinum toxin A for the treatment of delayed gastric emptying. Am J Gastroenterol. 2008;103(2):416-23.

26. Witteman BP, Conchillo JM, Rinsma NF, Betzel B, Peeters A, Koek GH, et al. Randomized controlled trial of transoral incisionless fundoplication vs. proton pump inhibitors for treatment of gastroesophageal reflux disease. Am J Gastroenterol. 2015;110(4):531-42.

27. Moonen A, Boeckxstaens G. Finding the Right Treatment for Achalasia Treatment: Risks, Efficacy, Complications. Curr Treat Options Gastroenterol. 2016;14(4):420-8.

28. Rohof WO, Hirsch DP, Kessing BF, Boeckxstaens GE. Efficacy of treatment for patients with achalasia depends on the distensibility of the esophagogastric junction. Gastroenterology. 2012;143(2):328-35.

29. Ponds FA, Bredenoord AJ, Kessing BF, Smout AJ. Esophagogastric junction distensibility identifies achalasia subgroup with manometrically normal esophagogastric junction relaxation. Neurogastroenterol Motil. 2017;29(1).

30. Smeets FG, Masclee AA, Keszthelyi D, Tjwa ET, Conchillo JM. Esophagogastric junction distensibility in the management of achalasia patients: relation to treatment outcome. Neurogastroenterol Motil. 2015;27(10):1495-503.

31. Carlson DA, Lin Z, Kahrilas PJ, Sternbach J, Donnan EN, Friesen L, et al. The functional lumen imaging probe detects esophageal contractility not observed with manometry in patients with achalasia. Gastroenterology. 2015;149(7):1742-51.

32. Pandolfino JE, de Ruigh A, Nicodeme F, Xiao Y, Boris L, Kahrilas PJ. Distensibility of the esophagogastric junction assessed with the functional lumen imaging probe (FLIP) in achalasia patients. Neurogastroenterol Motil. 2013;25(6):496-501.

33. Vaezi MF, Pandolfino JE, Vela MF. ACG clinical guideline: diagnosis and management of achalasia. Am J Gastroenterol. 2013;108(8):1238-49; quiz 50.

34. Eckardt VF, Aignherr C, Bernhard G. Predictors of outcome in patients with achalasia treated by pneumatic dilation. Gastroenterology. 1992;103(6):1732-8.

35. Eckardt VF, Gockel I, Bernhard G. Pneumatic dilation for achalasia: late results of a prospective follow up investigation. Gut. 2004;53(5):629-33.

36. Hulselmans M, Vanuytsel T, Degreef T, Sifrim D, Coosemans W, Lerut T, et al. Long-term outcome of pneumatic dilation in the treatment of achalasia. Clin Gastroenterol Hepatol. 2010;8(1):30-5.

37. Rohof WO, Lei A, Boeckxstaens GE. Esophageal stasis on a timed barium esophagogram predicts recurrent symptoms in patients with long-standing achalasia. Am J Gastroenterol. 2013;108(1):49-55.

38. Vaezi MF, Baker ME, Richter JE. Assessment of esophageal emptying post-pneumatic dilation: use of the timed barium esophagram. Am J Gastroenterol. 1999;94(7):1802-7.

39. Vaezi MF, Baker ME, Achkar E, Richter JE. Timed barium oesophagram: better predictor of long term success after pneumatic dilation in achalasia than symptom assessment. Gut. 2002;50(6):765-70.

40. Rieder E, Swanstrom LL, Perretta S, Lenglinger J, Riegler M, Dunst CM. Intraoperative assessment of esophagogastric junction distensibility during per oral endoscopic myotomy (POEM) for esophageal motility disorders. Surg Endosc. 2013;27(2):400-5.

41. Ngamruengphong S, von Rahden BH, Filser J, Tyberg A, Desai A, Sharaiha RZ, et al. Intraoperative measurement of esophagogastric junction cross-sectional area by impedance planimetry correlates with clinical outcomes of peroral endoscopic myotomy for achalasia: a multicenter study. Surg Endosc. 2016;30(7):2886-94. 
42. Familiari P, Gigante G, Marchese M, Boskoski I, Bove V, Tringali A, et al. EndoFLIP system for the intraoperative evaluation of peroral endoscopic myotomy. United European Gastroenterol J. 2014;2(2):77-83.

43. Verlaan T, Rohof WO, Bredenoord AJ, Eberl S, Rosch T, Fockens P. Effect of peroral endoscopic myotomy on esophagogastric junction physiology in patients with achalasia. Gastrointest Endosc. 2013;78(1):39-44.

44. Teitelbaum EN, Sternbach JM, El Khoury R, Soper NJ, Pandolfino JE, Kahrilas PJ, et al. The effect of incremental distal gastric myotomy lengths on EGJ distensibility during POEM for achalasia. Surg Endosc. 2016;30(2):745-50.

45. Teitelbaum EN, Soper NJ, Pandolfino JE, Kahrilas PJ, Hirano I, Boris L, et al. Esophagogastric junction distensibility measurements during Heller myotomy and POEM for achalasia predict postoperative symptomatic outcomes. Surg Endosc. 2015;29(3):522-8.

46. DeHaan RK, Frelich MJ, Gould JC. Intraoperative Assessment of Esophagogastric Junction Distensibility During Laparoscopic Heller Myotomy. Surg Laparosc Endosc Percutan Tech. 2016;26(2):137-40.

47. Ilczyszyn A, Hamaoui K, Cartwright J, Botha A. Intraoperative distensibility measurement during laparoscopic Heller's myotomy for achalasia may reduce the myotomy length without compromising patient outcome. Dis Esophagus. 2016;29(5):455-62.

48. Wu PI, Szczesniak MM, Craig PI, Choo L, Engelman J, Terkasher B, et al. Novel intra-procedural distensibility measurement accurately predicts immediate outcome of pneumatic dilatation for idiopathic achalasia. Am J Gastroenterol. 2018;113(2):205-12.

49. Ponds FA, Oors JM, Smout A, Bredenoord AJ. Rapid drinking challenge during high-resolution manometry is complementary to timed barium esophagogram for diagnosis and follow-up of achalasia. Neurogastroenterol Motil. 2018:e13404.

50. Carlson DA, Lin Z, Kahrilas PJ, Sternbach J, Hungness ES, Soper NJ, et al. High-resolution impedance manometry metrics of the esophagogastric junction for the assessment of treatment response in achalasia. Am J Gastroenterol. 2016;111(12):1702-10.

51. Khoury RE, Sternbach JM, Teitelbaum EN, Soper NJ, Hungness ES. Esophagogastric junction distensibility measured by a functional lumen imaging probe with incremental gastric myotomy lengths in achalasia. Surg Endosc. 2016;30(5):2139-40.

52. Kappelle WF, Bogte A, Siersema PD. Hydraulic dilation with a shape-measuring balloon in idiopathic achalasia: a feasibility study. Endoscopy. 2015;47(11):1028-34.

53. Ip S, Tatsioni A, Conant A, Karagozian R, Fu L, Chew P, et al. Predictors of clinical outcomes following fundoplication for gastroesophageal reflux disease remain insufficiently defined: a systematic review. Am J Gastroenterol. 2009;104(3):752-8; quiz 9.

54. Morgenthal CB, Lin E, Shane MD, Hunter JG, Smith CD. Who will fail laparoscopic Nissen fundoplication? Preoperative prediction of long-term outcomes. Surg Endosc. 2007;21(11):1978-84.

55. Cadiere GB, Buset M, Muls V, Rajan A, Rosch T, Eckardt AJ, et al. Antireflux transoral incisionless fundoplication using EsophyX: 12-month results of a prospective multicenter study. World J Surg. 2008;32(8):1676-88.

56. Testoni PA, Vailati C, Testoni S, Corsetti M. Transoral incisionless fundoplication (TIF 2.0) with EsophyX for gastroesophageal reflux disease: long-term results and findings affecting outcome. Surg Endosc. 2012;26(5):1425-35.

57. Testoni PA, Testoni S, Mazzoleni G, Vailati C, Passaretti S. Long-term efficacy of transoral incisionless fundoplication with Esophyx (Tif 2.0) and factors affecting outcomes in GERD patients followed for up to 6 years: a prospective single-center study. Surg Endosc. 2015;29(9):2770-80.

58. Pandolfino JE, Curry J, Shi G, Joehl RJ, Brasseur JG, Kahrilas PJ. Restoration of normal distensive characteristics of the esophagogastric junction after fundoplication. Ann Surg. 2005;242(1):43-8.

59. Blom D, Bajaj S, Liu J, Hofmann C, Rittmann T, Derksen T, et al. Laparoscopic Nissen fundoplication decreases gastroesophageal junction distensibility in patients with gastroesophageal reflux disease. J Gastrointest Surg. 2005;9(9):1318-25.

60. DeHaan RK, Davila D, Frelich MJ, Gould JC. Esophagogastric junction distensibility is greater following Toupet compared to Nissen fundoplication. Surg Endosc. 2017;31(1):193-8. 
61. Kessing BF, Bredenoord AJ, Schijven MP, van der Peet DL, van Berge Henegouwen MI, Smout AJ. Longterm effects of anti-reflux surgery on the physiology of the esophagogastric junction. Surg Endosc. 2015;29(12):3726-32.

62. Kwiatek MA, Pandolfino JE, Hirano I, Kahrilas PJ. Esophagogastric junction distensibility assessed with an endoscopic functional luminal imaging probe (EndoFLIP). Gastrointest Endosc. 2010;72(2):272-8.

63. Tucker E, Sweis R, Anggiansah A, Wong T, Telakis E, Knowles K, et al. Measurement of esophagogastric junction cross-sectional area and distensibility by an endolumenal functional lumen imaging probe for the diagnosis of gastro-esophageal reflux disease. Neurogastroenterol Motil. 2013;25(11): 904-10.

64. Chen JW, Rubenstein JH. Esophagogastric junction distensibility assessed using the functional lumen imaging probe. World J Gastroenterol. 2017;23(7):1289-97.

65. Rinsma NF, Smeets FG, Bruls DW, Kessing BF, Bouvy ND, Masclee AA, et al. Effect of transoral incisionless fundoplication on reflux mechanisms. Surg Endosc. 2014;28(3):941-9.

66. Smeets FG, Keszthelyi D, Bouvy ND, Masclee AA, Conchillo JM. Does Measurement of Esophagogastric Junction Distensibility by EndoFLIP Predict Therapy- responsiveness to Endoluminal Fundoplication in Patients With Gastroesophageal Reflux Disease? J Neurogastroenterol Motil. 2015;21(2):255-64.

67. Carlson DA, Kathpalia P, Craft J, Tye M, Lin Z, Kahrilas PJ, et al. The relationship between esophageal acid exposure and the esophageal response to volumetric distention. Neurogastroenterol Motil. 2018;30(3).

68. Trudgill NJ, Riley SA. Transient lower esophageal sphincter relaxations are no more frequent in patients with gastroesophageal reflux disease than in asymptomatic volunteers. Am J Gastroenterol. 2001;96(9):2569-74.

69. Bredenoord AJ, Draaisma WA, Weusten BL, Gooszen HG, Smout AJ. Mechanisms of acid, weakly acidic and gas reflux after anti-reflux surgery. Gut. 2008;57(2):161-6.

70. Beaumont H, Bennink RJ, de Jong J, Boeckxstaens GE. The position of the acid pocket as a major risk factor for acidic reflux in healthy subjects and patients with GORD. Gut. 2010;59(4):441-51.

71. Herbella FA, Vicentine FP, Del Grande JC, Patti MG. Postprandial proximal gastric acid pocket in patients after laparoscopic Nissen fundoplication. Surg Endosc. 2011;25(10):3198-201.

72. Kim MP, Meisenbach LM, Chan EY. Tailored Fundoplication With Endoluminal Functional Lumen Imaging Probe Allows for Successful Minimally Invasive Hiatal Hernia Repair. Surg Laparosc Endosc Percutan Tech. 2018;28(3):178-82.

73. Ilczyszyn A, Botha AJ. Feasibility of esophagogastric junction distensibility measurement during Nissen fundoplication. Dis Esophagus. 2014;27(7):637-44.

74. Vanheel H, Carbone F, Valvekens L, Simren M, Tornblom H, Vanuytsel T, et al. Pathophysiological Abnormalities in Functional Dyspepsia Subgroups According to the Rome III Criteria. Am J Gastroenterol. 2017;112(1):132-40.

75. Vakil NB, Howden CW, Moayyedi P, Tack J. White Paper AGA: Functional Dyspepsia. Clin Gastroenterol Hepatol. 2017;15(8):1191-4.

76. Hsu YC, Liou JM, Yang TH, Hsu WL, Lin HJ, Wu HT, et al. Proton pump inhibitor versus prokinetic therapy in patients with functional dyspepsia: is therapeutic response predicted by Rome III subgroups? J Gastroenterol. 2011;46(2):183-90.

77. Moayyedi PM, Lacy BE, Andrews CN, Enns RA, Howden CW, Vakil N. ACG and CAG Clinical Guideline: Management of Dyspepsia. Am J Gastroenterol. 2017;112(7):988-1013.

78. Ang D. Measurement of gastric accommodation: a reappraisal of conventional and emerging modalities. Neurogastroenterol Motil. 2011;23(4):287-91.

79. Janssen P, Verschueren S, Ly HG, Vos R, Van Oudenhove L, Tack J. Intragastric pressure during food intake: a physiological and minimally invasive method to assess gastric accommodation. Neurogastroenterol Motil. 2011;23(4):316-22, e153-4.

80. Bolling-Sternevald E, Lauritsen K, Talley NJ, Junghard O, Glise H. Is it possible to predict treatment response to a proton pump inhibitor in functional dyspepsia? Aliment Pharmacol Ther. 2003;18(1):117-24. 
81. Talley NJ, Vakil N, Lauritsen K, van Zanten SV, Flook N, Bolling-Sternevald E, et al. Randomizedcontrolled trial of esomeprazole in functional dyspepsia patients with epigastric pain or burning: does a 1-week trial of acid suppression predict symptom response? Aliment Pharmacol Ther. 2007;26(5):673-82.

82. Mearin F, Camilleri M, Malagelada JR. Pyloric dysfunction in diabetics with recurrent nausea and vomiting. Gastroenterology. 1986;90(6):1919-25.

83. Lacy BE, Crowell MD, Schettler-Duncan A, Mathis C, Pasricha PJ. The treatment of diabetic gastroparesis with botulinum toxin injection of the pylorus. Diabetes Care. 2004;27(10):2341-7.

84. Camilleri M, Parkman HP, Shafi MA, Abell TL, Gerson L, American College of G. Clinical guideline: management of gastroparesis. Am J Gastroenterol. 2013;108(1):18-37; quiz 8.

85. Bromer MQ, Friedenberg F, Miller LS, Fisher RS, Swartz K, Parkman HP. Endoscopic pyloric injection of botulinum toxin A for the treatment of refractory gastroparesis. Gastrointest Endosc. 2005;61(7):8339.

86. Coleski R, Anderson MA, Hasler WL. Factors associated with symptom response to pyloric injection of botulinum toxin in a large series of gastroparesis patients. Dig Dis Sci. 2009;54(12):2634-42.

87. Janssen $\mathrm{P}$, Harris MS, Jones M, Masaoka T, Farre R, Tornblom H, et al. The relation between symptom improvement and gastric emptying in the treatment of diabetic and idiopathic gastroparesis. Am J Gastroenterol. 2013;108(9):1382-91.

88. Snape WJ, Lin MS, Agarwal N, Shaw RE. Evaluation of the pylorus with concurrent intraluminal pressure and EndoFLIP in patients with nausea and vomiting. Neurogastroenterol Motil. 2016;28(5):758-64.

89. Gourcerol G, Tissier F, Melchior C, Touchais JY, Huet E, Prevost G, et al. Impaired fasting pyloric compliance in gastroparesis and the therapeutic response to pyloric dilatation. Aliment Pharmacol Ther. 2015;41(4):360-7.

90. Malik Z, Sankineni A, Parkman HP. Assessing pyloric sphincter pathophysiology using EndoFLIP in patients with gastroparesis. Neurogastroenterol Motil. 2015;27(4):524-31.

91. Jacques J, Pagnon L, Hure F, Legros R, Crepin S, Fauchais AL, et al. Peroral endoscopic pyloromyotomy is efficacious and safe for refractory gastroparesis: prospective trial with assessment of pyloric function. Endoscopy. 2019;51(1):40-9.

92. Nicodeme F, Hirano I, Chen J, Robinson K, Lin Z, Xiao Y, et al. Esophageal distensibility as a measure of disease severity in patients with eosinophilic esophagitis. Clin Gastroenterol Hepatol. 2013;11(9):1101-7 e1.

93. Kwiatek MA, Hirano I, Kahrilas PJ, Rothe J, Luger D, Pandolfino JE. Mechanical properties of the esophagus in eosinophilic esophagitis. Gastroenterology. 2011;140(1):82-90.

94. Lin Z, Kahrilas PJ, Xiao Y, Nicodeme F, Gonsalves N, Hirano I, et al. Functional luminal imaging probe topography: an improved method for characterizing esophageal distensibility in eosinophilic esophagitis. Therap Adv Gastroenterol. 2013;6(2):97-107.

95. Carlson DA, Kahrilas PJ, Lin Z, Hirano I, Gonsalves N, Listernick Z, et al. Evaluation of Esophageal Motility Utilizing the Functional Lumen Imaging Probe. Am J Gastroenterol. 2016;111(12):1726-35. 


\section{Addendum}

Summary

Samenvatting

Valorization

List of publications

Dankwoord

Curriculum vitae 



\section{Summary}

Functional gastrointestinal disorders (FGIDs) are some of the most common digestive disorders affecting at least one-third of the general population. Functional dyspepsia (FD) is a FGID with an estimated prevalence of $11-29 \%$ in the general population. FD is a symptom-based diagnosis and due to the lack of available biomarkers or objective outcome measures, evaluation of treatment efficacy is based on the use of patientreported outcome measures (PROMs). The first part of this thesis aims to describe the current available PROMs for symptom assessment in patients with functional dyspepsia, and the development of a novel PROM based on the Experience Sampling Method (ESM). Besides the challenging evaluation of treatment efficacy with PROMs, the incompletely understood multifactorial pathophysiology of several functional and somatic upper gastrointestinal disorders including FD, gastroparesis, gastroesophageal reflux disease (GERD), and achalasia limits the development of effective treatment options. Moreover, in the last decades a shift has been observed from invasive, surgical procedures to less invasive (endoscopic) treatment options. These less invasive (endoscopic) procedures seem to share the characteristic of beneficial treatment outcome limited to a subgroup of patients which underlines the need for adequate patient selection. Therefore, the second part of this thesis aims to evaluate the use of diagnostic modalities for assessment of treatment efficacy and to predict treatment outcome in patients with upper gastrointestinal disorders treated with (endoscopic) minimally invasive procedures.

\section{Part I Patient-reported outcome measures in functional dyspepsia}

In chapter $\mathbf{2}$ we performed an extensive systematic literature search to identify all available (disease-specific) PROMs for symptom assessment in FD patients. Forty articles were included in this review, and the PROMS were divided into two categories: (1) fourteen unidimensional outcome measures evaluating gastrointestinal symptoms only, and (2) six multidimensional outcome measures evaluating both gastrointestinal symptoms and additional domains including quality of life or impact of symptoms on emotional functioning. Psychometric validation properties, strengths and limitations of the PROMs were described. The majority of the described outcome measures proved to be reliable and valid, although in a substantial part no assessment of responsiveness has been performed limiting its use in clinical trials. Altogether, none of the current available outcome measures fulfills all validation criteria defined by regulatory authorities as several assumptions are not met: (a) lack of assessment of all FD core symptoms, (b) use of investigator-administered instead of the recommended patient-administered questionnaires, and (c) lack of validation of the PROM in the target population of well-defined FD patients according to Rome criteria. In addition, several limitations of retrospective, end-of-day paper questionnaires were mentioned including recall bias, ecological bias and non-compliance. 
Due to the lack of one universal accepted PROM for symptom assessment in FD patients, we described in chapter 3 the development of a novel PROM based on the Experience Sampling Methodology. In line with the recommendation of the Food and Drug Administration (FDA), patient focus group interviews were performed after development of a conceptual framework. Fourteen FD patients according to Rome IV criteria participated in focus group interviews and discussed their (gastrointestinal) symptoms, potential triggers for symptom generation and/or severity, as well as the influence of their symptoms on daily life. Psychological factors, context, nutrition, medication and substance use were identified as potential symptom triggers. Moreover, they criticized 59 items selected from previously used outcome measures, and discussed practical issues of the experience sampling technology. After focus group interviews, a teleconference meeting with international experts in the field was organized to discuss the relevance of the individual items and a definitive draft with 33-items was created. Patients' understanding of this novel ESM-based PROM was evaluated during six cognitive grasp interviews, and led to some minor linguistic changes. Additional evaluation of psychometric validation properties including assessment of reliability, validity and responsiveness, has to be performed before use of this novel ESM-based PROM in clinical practice.

\section{Part II Prediction of treatment outcome in upper gastrointestinal disorders}

\section{Achalasia}

Achalasia is a primary esophageal motility disorder characterized by insufficient relaxation of the lower esophageal sphincter, and loss of esophageal peristalsis. Previous studies with the novel endoscopic functional luminal imaging probe (EndoFLIP) described reduced esophagogastric junction (EGJ) distensibility in newly diagnosed achalasia patients compared to healthy controls, with an increase in distensibility after treatment. Due to the declining success rates of pneumatic balloon dilation (PD) over time, follow-up to evaluate treatment efficacy and need for retreatment are necessary although the method for functional testing is still a matter of debate. In chapter 4, we evaluated the effect of PD in 26 newly diagnosed achalasia patients. Newly diagnosed achalasia patients had reduced EGJ distensibility compared to fifteen healthy controls ( $50 \mathrm{ml}$ EndoFLIP distension volume: 0.9 (0.7-1.5) vs. 3.4 (2.7$\left.4.2) \mathrm{mm}^{2} / \mathrm{mmHg}, \mathrm{p}<0.01\right)$. The $10^{\text {th }}$ percentile of EGJ distensibility in healthy controls was used to determine a cutoff value for normality which was $2.1 \mathrm{~mm}^{2} / \mathrm{mmHg}$, and 96\% of newly diagnosed achalasia patients had EGJ distensibility below this cutoff value. Treatment with PD resulted in a significant direct increase in EGJ distensibility (50ml EndoFLIP distension volume: $0.9(0.7-1.5)$ to $4.2(3.0-5.7) \mathrm{mm}^{2} / \mathrm{mmHg}, \mathrm{p}<0.01$ ). At one year follow-up, $73 \%$ of patients had a good clinical outcome based on the 
Eckardt symptom score. Esophagogastric junction distensibility did not differ between patients with good and poor clinical outcome both before and after PD. In addition, only 1 patient with clinical treatment failure had an EGJ distensibility below the cutoff value for normality directly after PD. As a consequence, assessment of EGJ distensibility by the EndoFLIP technique to predict treatment outcome and identify patients with need for retreatment seems limited based on the results of the present study, although conflicting results have been described in literature. An attractive application of the EndoFLIP technique in achalasia patients seems however to be its intraoperative use during laparoscopic Heller myotomy or peroral endoscopic myotomy to evaluate the effect of treatment on EGJ distensibility to improve treatment outcome and prevent development of long-term complications.

\section{Gastroesophageal reflux disease}

Gastroesophageal reflux disease (GERD) is considered a multifactorial disorder, and several proposed pathophysiological mechanisms are EGJ dysfunction, Transient Lower Esophageal Sphincter Relaxations (TLESRs) and the acid pocket. Use of acid suppressive medication is the first line therapy for GERD patients, but in case of persistent symptoms surgical treatment can be considered. Several long-term complications of the traditional (laparoscopic) Nissen fundoplication led to the development of less invasive (endoscopic) treatment options. Transoral Incisionless Fundoplication (TIF) using the Esophyx device is probably most closely related to surgical fundoplication. In chapter $\mathbf{5}$ we evaluated the effect of TIF on TLESRs using combined high-resolution manometry and $\mathrm{pH}$-impedance monitoring. After TIF, a significant reduction in both the number of TLESRs (from 16.8 \pm 1.5 to $9.2 \pm 1.3, p<0.01$ ) and the number of TLESRs associated with liquid reflux was found (from $11.1 \pm 1.6$ to $5.6 \pm 0.6(p<0.01)$. However, the proportion of TLESRs associated with liquid-containing reflux was not reduced after TIF. Moreover, in chapter 6 the influence of TIF on EGJ distensibility was evaluated using the EndoFLIP technique. Forty-two GERD patients were treated with TIF and 21 patients were considered objective treatment responders with normalization of esophageal acid exposure time at six months followup. Patients with normalization of esophageal acid exposure time had lower preoperative esophageal acid exposure time and EGJ distensibility before TIF compared to non-responders (30 ml EndoFLIP distension volume: 1.5 (1.1-2.1) vs 2.5 $\left.(1.7-3.4) \mathrm{mm}^{2} / \mathrm{mmHg}, \mathrm{p}<0.05\right)$, and both parameters were independent predictors of treatment outcome. Based on these results, patients with preoperative EGJ distensibility $<2.3 \mathrm{~mm}^{2} / \mathrm{mmHg}$ and esophageal acid exposure time $<11 \%$ seemed to be the best candidates for TIF. However, in contrast to objective treatment outcome both variables were not associated with clinical symptomatic outcome after TIF, and no association was found between objective treatment outcome and clinical treatment outcome. Moreover, in a subgroup of 25 patients EndoFLIP measurements 
were repeated direct postoperative and at six months follow-up. Although a significant decrease was found direct postoperative, no change in EGJ distensibility was found at six months follow-up compared to preoperative EGJ distensibility. As there was a significant reduction in esophageal acid exposure time and the number of reflux episodes six months after TIF, we have to consider other mechanisms in contrast to a change in EGJ distensibility as potential antireflux mechanisms. Potential mechanisms are for example small hiatal hernia repair and influence of TIF on the acid pocket. Consequently, the role of EndoFLIP in the preoperative evaluation of GERD patients seems limited. The EndoFLIP might however be an attractive tool for intraoperative use during Nissen or Toupet fundoplication in order to create optimal reflux control without development of long-term complications due to creation of a too tight wrap.

\section{Gastroparesis}

Gastroparesis is defined by delayed gastric emptying without evidence for a mechanical obstruction. Treatment of gastroparesis remains challenging at least in part due to the multifactorial and up until now incompletely understood pathophysiology. First line therapy consists of dietary advices combined with use of prokinetic agents. In case of refractory symptoms, endoscopic procedures can be considered including use of pyloric botulinum toxin injection. In chapter $\mathbf{7}$ we assessed clinical outcome after treatment of 40 gastroparesis patients with botulinum toxin therapy. Fourteen patients (35\%) were considered symptom-responders, whereas $65 \%$ had no beneficial symptomatic effect of botulinum toxin treatment, and after a mean follow-up of 40 months the majority of patients received additional nutritional interventions. As only a subgroup of patients has a beneficial treatment outcome together with the high costs and need for repeated botulinum toxin injections, adequate patient selection is warranted. However, no differences were found in manometric and scintigraphic variables between symptom responders and non-responders. In addition, patient characteristics, manometric and scintigraphic variables were not able to predict treatment outcome, limiting the use of variables of both antroduodenal manometry and scintigraphy as predictor of treatment outcome. For the future, assessment of pyloric distensibility with the EndoFLIP technique seems to be a parameter of interest, as recent studies described impaired pyloric distensibility in a subgroup of gastroparesis patients. Patients with reduced pyloric distensibility might be potential candidates for novel endoscopic treatment options including the endoscopic gastric peroral pyloromyotomy (G-POEM). 


\section{Samenvatting}

Functionele maag- en darmziekten behoren tot de meest voorkomende aandoeningen van het spijsverteringsstelsel. Functionele maag- en darmziekten treffen tenminste $1 / 3^{e}$ deel van de algemene bevolking. Functionele dyspepsie is een voorbeeld van een functionele maag- en darmziekte met een geschatte prevalentie van $11-29 \%$. Functionele dyspepsie is een ziekte die op basis van symptomen wordt gediagnosticeerd. Door het gebrek aan beschikbare biomarkers of objectieve uitkomstmaten is het evalueren van de behandeluitkomst gebaseerd op het gebruik van vragenlijsten, welke ook wel patiënt gerapporteerde uitkomstmaten (PROMs) worden genoemd. Het eerste gedeelte van dit proefschrift heeft tot doel om de huidige beschikbare PROMs te beschrijven welke worden gebruikt om symptomen bij patiënten met functionele dyspepsie te beoordelen, en om de ontwikkeling van een nieuwe PROM te beschrijven welke is gebaseerd op de Experience Sampling Method (ESM).

Naast het feit dat het beoordelen van het behandeleffect middels PROMs uitdagend is, zorgt de onvolledig begrepen en multifactoriële pathofysiologie van verschillende functionele en somatische aandoeningen van het bovenste deel van het maag-darmstelsel (o.a. functionele dyspepsie, gastroparese, refluxziekte, en achalasie) ervoor dat het moeilijk is om effectieve behandelingen te ontwikkelen. Bovendien heeft er de afgelopen decennia een verschuiving plaatsgevonden van invasieve operatieve behandelingen naar minder invasieve (endoscopische) behandelopties. Deze minder invasieve (endoscopische) behandelopties lijken een belangrijk punt gemeen te hebben, namelijk dat ze effectief lijken te zijn in een selecte subgroep van patiënten. Het is daarom belangrijk om patiënten op adequate wijze te selecteren voor verschillende behandelopties. Het tweede deel van dit proefschrift heeft daarom tot doel om het gebruik van verschillende diagnostische testen te onderzoeken welke gebruikt kunnen worden om het effect van behandelingen te beoordelen, en om te voorspellen welke patiënten behandeld kunnen worden met de minimaal invasieve (endoscopische) procedures.

\section{Deel I Patiënt gerapporteerde uitkomstmaten bij functionele dyspepsie}

In hoofdstuk 2 hebben we een uitgebreide systematische zoekstrategie uitgevoerd waarbij we alle beschikbare PROMs hebben gevonden welke momenteel worden gebruikt voor het beoordelen van symptomen bij patiënten met functionele dyspepsie. Veertig artikels werden geïncludeerd in deze systematische review, en de PROMs werden verdeeld in twee categorieën: (1) veertien unidimensionele uitkomstmaten die uitsluitend maag-darm symptomen beoordelen, en (2) zes multidimensionele uitkomstmaten die zowel maag-darm symptomen beoordelen alsmede aanvullende domeinen zoals kwaliteit van leven of de impact van symptomen 
op emotioneel welbevinden. Psychometrische validatie eigenschappen evenals sterktes en zwaktes van deze PROMs werden beschreven. Het merendeel van de beschreven vragenlijsten bleek betrouwbaar en valide te zijn. Een substantieel deel van de vragenlijsten heeft echter geen onderzoek gedaan naar het vermogen van de vragenlijst om verschillen in symptomen in de loop van de tijd te detecteren. Hierdoor is de toepasbaarheid van deze vragenlijsten in de huidige klinische praktijk beperkt. Concluderend kan worden gesteld dat geen enkele van de huidige beschikbare uitkomstmaten voldoet aan alle validatie criteria zoals die worden gedefinieerd door regelgevende instanties. Dit komt doordat er aan verschillende aannames niet wordt voldaan: (a) niet alle hoofdsymptomen van functionele dyspepsie worden beoordeeld met de vragenlijsten, (b) er wordt gebruik gemaakt van vragenlijsten die door de onderzoeker of arts worden ingevuld, terwijl wordt aangeraden dat patiënten de vragenlijsten zelfstandig invullen, en (c) de vragenlijsten worden niet onderzocht in de doelpopulatie bestaande uit goed gedefinieerde patiënten met functionele dyspepsie op basis van de Rome criteria. Het merendeel van de vragenlijsten wordt verder eenmalig aan het einde van de dag ingevuld. Hierbij wordt aan patiënten gevraagd om de klachten over de afgelopen dag te beoordelen, dit wordt retrospectief genoemd. Deze retrospectieve, einde-van-de-dag vragenlijsten hebben ook enkele beperkingen welke vermeld dienen te worden. Hiertoe behoren recall bias, ecologische bias, noncompliance.

Gezien het gebrek aan een universeel geaccepteerde PROM voor beoordeling van symptomen bij patiënten met functionele dyspepsie, beschrijven we in hoofdstuk $\mathbf{3}$ de ontwikkeling van een nieuwe PROM die is gebaseerd op de Experience Sampling Methodology (ESM). In overeenkomst met de aanbevelingen van regelgevende instanties werd een schema gemaakt met alle (symptoom) categorieën welke geïncludeerd moeten worden in de nieuwe vragenlijst. Nadien hebben we focus groep interviews georganiseerd met dyspepsie patiënten. Veertien patiënten met functionele dyspepsie op basis van de Rome IV criteria hebben deelgenomen aan deze focus groep interviews. Tijdens deze interviews spraken de patiënten over hun (maagdarm) symptomen, mogelijke factoren die de klachten kunnen opwekken en/of de ernst van de klachten kunnen beïnvloeden ('triggers'), alsmede de invloed van hun klachten op het dagelijks functioneren. Psychologische factoren, de context/omgeving, voeding, medicatie en middelengebruik werden gezien als factoren die de klachten kunnen veroorzaken en/of verergeren. Tevens bediscussieerden de deelnemers 59 items welke werden geselecteerd uit eerder gebruikte vragenlijsten, alsmede praktische punten van de ESM technologie. Na afronding van deze focus groep interviews werd een teleconferentie bijeenkomst georganiseerd waaraan verschillende internationale experts op het gebied van functionele maag- en darmziekten deelnamen. Tijdens deze teleconferentie bijeenkomst werd de relevantie van de geselecteerde items bediscussieerd en uiteindelijk werd een definitieve versie van de vragenlijst gemaakt. Deze definitieve versie van de vragenlijst bevat 33-items. 
Nadien werden zes cognitieve interviews afgenomen bij patiënten met functionele dyspepsie om te beoordelen of zij alle items van de nieuwe vragenlijst begrepen. Deze cognitieve interviews hebben geleid tot enkele taalkundige aanpassingen in de vragenlijst. In toekomstige studies moet de nieuw ontwikkelde vragenlijst verder geëvalueerd worden alvorens de vragenlijst kan worden gebruikt in de dagelijkse praktijk. In toekomstige studies moet onder andere gekeken worden naar de betrouwbaarheid, validiteit en het vermogen van de vragenlijst om verandering in symptomen in de loop van de tijd te identificeren.

Deel II Voorspellen van behandeluitkomst bij aandoeningen van het bovenste gedeelte van het maag-darmstelsel

\section{Achalasie}

Achalasie is een motoriekstoornis van de slokdarm. De ziekte wordt gekenmerkt door onvoldoende ontspanning van de onderste slokdarmsfincter in combinatie met verlies van peristaltiek in de slokdarm. Eerdere studies die gebruik hebben gemaakt van de nieuwe EndoFLIP techniek hebben beschreven dat de oprekbaarheid van de slokdarmmaagovergang afgenomen is bij nieuw gediagnosticeerde patiënten met achalasie ten opzichte van gezonde proefpersonen. Tevens zorgt behandeling van patiënten met achalasie voor een toename van de oprekbaarheid van de slokdarm-maag overgang. Een van de behandelopties voor patiënten met achalasie is pneumodilatatie waarbij een ballon ter hoogte van de slokdarm-maagovergang met kracht wordt opgeblazen. Onderzoeken hebben laten zien dat in de loop van de tijd er een afname van het succespercentage wordt gezien na deze behandeling. Daarom is het belangrijk om patiënten te vervolgen na de behandeling om te beoordelen hoe effectief de behandeling is geweest en om te beoordelen of er noodzaak is voor aanvullende behandelingen. De methode of test waarmee patiënten vervolgd moeten worden is echter tot op heden onderwerp van discussie. In hoofdstuk 4 beschrijven we 26 nieuw gediagnosticeerde achalasie patiënten die worden behandeld met pneumodilatatie. De achalasie patiënten hadden een lagere oprekbaarheid van de slokdarm-maag overgang ten opzichte van 15 gezonde proefpersonen $(50 \mathrm{ml}$ EndoFLIP volume: 0.9 $(0.7-1.5)$ vs. $\left.3.4(2.7-4.2) \quad \mathrm{mm}^{2} / \mathrm{mmHg}, \mathrm{p}<0.01\right)$. Het $10^{\mathrm{e}}$ percentiel van de oprekbaarheid bij gezonde proefpersonen werd gebruikt om een afkapwaarde voor normaal te definiëren. Deze afkapwaarde bedroeg $\geq 2.1 \mathrm{~mm}^{2} / \mathrm{mmHg}$, en $96 \%$ van de nieuw gediagnosticeerde achalasie patiënten had een waarde die onder deze afkapwaarde was gelegen. Behandeling van de patiënten met pneumodilatatie zorgt voor een toename van de oprekbaarheid (50ml EndoFLIP volume: van 0.9 (0.7-1.5) naar $\left.4.2(3.0-5.7) \mathrm{mm}^{2} / \mathrm{mmHg}, \mathrm{p}<0.01\right)$. Eén jaar na de behandeling heeft $73 \%$ van de patiënten een goede behandeluitkomst aangezien zij weinig symptomen rapporteren. Er werd geen verschil in de oprekbaarheid van de slokdarm-maagovergang 
waargenomen direct voor of na de pneumodilatatie tussen patiënten die een goede of slechte behandeluitkomst hebben 1 jaar na therapie. Tevens heeft slechts 1 patiënt met een slechte behandeluitkomst een oprekbaarheid van de slokdarmmaagovergang die onder de afkapwaarde van normaal ligt direct na het verrichten van de pneumodilatatie. Hierdoor lijkt bepaling van de oprekbaarheid van de slokdarm-maagovergang slechts beperkte waarde te hebben voor het voorspellen van de behandeluitkomst en het identificeren van patiënten die behoefte hebben aan aanvullende behandelingen. In de beschreven literatuur worden hierover echter tegenstrijdige resultaten weergegeven. Een mogelijke aantrekkelijke toepassing van de EndoFLIP techniek bij achalasie patiënten is het intraoperatief gebruik tijdens een laparoscopische Heller myotomy of peroral endoscopic myotomy (POEM) om tijdens de operatie direct het effect van de behandeling op de oprekbaarheid van de slokdarm-maagovergang te evalueren. Hierdoor kan mogelijk de behandeluitkomst verbeteren terwijl lange-termijn complicaties kunnen worden voorkomen.

\section{Gastro-oesofageale refluxziekte}

Gastro-oesofageale refluxziekte (GERD) wordt gezien als een multifactoriële ziekte aangezien wordt verondersteld dat meerdere mechanismen bijdragen aan dit ziektebeeld. Een aantal veronderstelde mechanismen zijn disfunctie van de slokdarmmaagovergang, Transient Lower Esophageal Sphincter Relaxations (TLESRs), en de zogenaamde 'zure pocket' in de maag. De eerstelijns behandeling voor GERD bestaat uit het gebruik van maagzuurremmers. Indien er echter sprake is van aanhoudende symptomen kan operatieve behandeling worden overwogen. Verschillende langetermijn complicaties van de traditionele (laparoscopische) Nissen fundoplicatie hebben geleid tot de ontwikkeling van minder invasieve (endoscopische) behandelopties. Transoral Incisionless Fundoplication (TIF) met behulp van het Esophyx apparaat is een techniek die waarschijnlijk het meest overeenkomt met de chirurgische fundoplicatie. In hoofdstuk 5 hebben we het effect van TIF beoordeeld op TLESRs door middel van een gecombineerde manometrie en impedantie meting. $\mathrm{Na}$ de TIF procedure wordt er een significante afname waargenomen van het aantal TLESRs (van 16.8 \pm 1.5 naar $9.2 \pm 1.3, p<0.01$ ), evenals een significante afname van het aantal TLESRs die geassocieerd zijn met vloeibare reflux (van $11.1 \pm 1.6$ naar $5.6 \pm 0.6$ $(p<0.01)$. Echter, er werd geen verandering waargenomen in het percentage van de TLESRs welke geassocieerd waren met vloeibare reflux. Verder beschrijven we in hoofdstuk 6 het effect van de TIF procedure op de oprekbaarheid van de slokdarmmaagovergang door middel van de EndoFLIP techniek. Tweeënveertig patiënten met GERD werden behandeld middels TIF en 21 patiënten werden beschouwd als patiënten met een gunstige behandeluitkomst aangezien er normalisatie van de zure expositie tijd in de slokdarm werd waargenomen 6 maanden na de behandeling. Patiënten met normalisatie van de zure reflux tijd in de slokdarm hadden een lagere 
preoperatieve zure reflux tijd en lagere oprekbaarheid van de slokdarmmaagovergang ten opzichte van patiënten die een ongunstige behandeluitkomst hadden (30ml EndoFLIP volume: $1.5(1.1-2.1)$ vs. $2.5(1.7-3.4) \mathrm{mm}^{2} / \mathrm{mmHg}, \mathrm{p}<0.05$ ). Zowel de preoperatieve zure reflux tijd als de oprekbaarheid van de slokdarmmaagovergang waren onafhankelijke voorspellers van de behandeluitkomst. Patiënten met een preoperatieve oprekbaarheid van de slokdarm-maagovergang $<2.3$ $\mathrm{mm}^{2} / \mathrm{mmHg}$ en zure reflux tijd $<11 \%$ lijken de beste kandidaten te zijn voor TIF. Echter, beide variabelen waren niet geassocieerd met symptomen na de TIF procedure, en er werd geen associatie waargenomen tussen de objectieve behandeluitkomst gebaseerd op zure reflux tijd, en subjectieve behandeluitkomst gebaseerd op symptomen. Verder werd in een subgroep van 25 patiënten de EndoFLIP meting direct na de TIF procedure herhaald, evenals 6 maanden na de TIF procedure. Hoewel er een directe significante afname van de oprekbaarheid van de slokdarm-maagovergang werd waargenomen na de TIF procedure, werd er geen verschil in oprekbaarheid gevonden 6 maanden na de procedure. Aangezien er wel sprake was van een significante afname van de zure reflux tijd en het aantal reflux episodes 6 maanden na TIF, moeten andere potentiele antireflux mechanismen overwogen worden in plaats van de verandering van oprekbaarheid van de slokdarmmaagovergang. Mogelijke mechanismen zijn bijvoorbeeld het herstel van een kleine middenrifbreuk, en invloed van TIF op de 'zure pocket' in de maag. Derhalve lijkt de rol van de EndoFLIP techniek in de preoperatieve evaluatie van GERD patiënten beperkt. De EndoFLIP is mogelijk echter wel een aantrekkelijke techniek voor intraoperatief gebruik tijdens Nissen of Toupet fundoplicatie om op deze manier optimale reflux controle te bewerkstelligen zonder het ontstaan van lange-termijn complicaties door te voorkomen dat de manchet te strak wordt gemaakt.

\section{Gastroparese}

Gastroparese is een ziektebeeld dat gekenmerkt wordt door vertraagde maaglediging zonder dat er aanwijzingen worden gevonden voor een mechanische obstructie. Behandeling van patiënten met gastroparese is uitdagend door de multifactoriële en tot op heden onvolledig begrepen pathofysiologie. Eerstelijns behandeling bestaat uit dieetadviezen in combinatie met gebruik van medicijnen die de maaglediging versnellen (prokinetica). Indien er ondanks deze behandeling echter sprake is van persisterende symptomen kunnen endoscopische behandelopties worden overwogen inclusief het injecteren van botulinum toxine (botox) in de sluitspier van de maag (pylorus). In hoofdstuk 7 beschrijven we het effect van behandeling met botulinum toxine in 40 patiënten met gastroparese. Veertien patiënten (35\%) hebben een goede klinische uitkomst en worden symtoom-responders genoemd, terwijl $65 \%$ van de patiënten onvoldoende effect heeft van botulinum toxine op hun klachten ('nonresponders'). Het merendeel van de patiënten heeft uiteindelijk ook ondersteuning op 
gebied van voeding nodig. Aangezien slechts een gedeelte van de patiënten een gunstige behandeluitkomst heeft, lijkt het selecteren van patiënten voor deze behandeling van essentieel belang. Dit mede gezien het feit dat behandeling met botulinum toxine duur is en er herhaaldelijke behandelingen noodzakelijk zijn door het tijdelijke effect van de therapie. In de studie werden echter geen verschillen waargenomen in parameters die zijn bepaald middels antroduodenale manometrie en scintigrafie tussen patiënten met goede en slechte behandeluitkomst. Verder waren zowel patiëntkenmerken, als parameters bepaald middels antroduodenale manometrie en scintigrafie niet in staat om de behandeluitkomst te voorspellen. In de toekomst is er mogelijk een rol weggelegd voor het beoordelen van de oprekbaarheid van de pylorus met behulp van de EndoFLIP techniek, aangezien recente studies hebben laten zien dat een subgroep van de gastroparese patiënten een gestoorde oprekbaarheid van de pylorus heeft. Patiënten met een afgenomen oprekbaarheid van de pylorus zijn mogelijke kandidaten voor nieuwe endoscopische behandelopties inclusief de endoscopische gastric peroral pyloromyotomy (G-POEM). 


\section{Valorization}

\section{Part I Patient-reported outcome measures in functional dyspepsia}

Functional dyspepsia is a common functional gastrointestinal disorder with an estimated prevalence of $11-29 \%$ in the general population. ${ }^{1}$ Due to this high prevalence together with the negative impact on daily life, dyspeptic complaints are associated with a considerable economic burden. First, (functional) dyspepsia is associated with reduced work productivity. In a large study from Brazil, $32 \%$ of patients with dyspepsia reported lost hours of work in the preceding week because of dyspeptic symptoms with an average of 2.6 hours per week. In addition, $78 \%$ of patients reported reduced work productivity, and the costs of the total work productivity impairment was $\$ 3570$ annually per employed dyspeptic patient. ${ }^{2}$ Besides the impaired work productivity, dyspepsia also increases health care costs. Results from a longitudinal 10-year follow-up study demonstrated that from 3266 included participants, 1738 (53\%) had dyspeptic symptoms at any point during this follow-up period. Seven hundred twenty-nine of patients with dyspeptic symptoms $(42 \%)$ consulted a primary care physician one or more times for their complaints. Sixty percent of patients seeking medical help for their dyspeptic complaints had been prescribed acid suppressant therapy. ${ }^{3}$ These results are in line with a population based cohort study including 206 subjects with dyspepsia. In total, $48 \%$ of patients consulted a physician for dyspeptic symptoms over 20 years. Of these patients, $13 \%$ was referred to a gastroenterologist after their first episode of dyspepsia, $65 \%$ had an upper gastrointestinal barium study, 49\% had an upper gastrointestinal endoscopy, and $91 \%$ received a dyspepsia related medical prescription. ${ }^{4}$ In addition, dyspeptic patients report reduced quality of life.

The high prevalence of dyspepsia together with the negative impact on work productivity and health care costs underline the need for (development of) effective treatment options. Treatment of dyspepsia is however challenging due to the multifactorial and incompletely understood pathophysiology. Moreover, symptom assessment is necessary to evaluate treatment efficacy due to the lack of biomarkers or objective outcome measures. In chapter $\mathbf{2}$ we performed a systematic literature search and identified 20 available outcome measures to assess dyspeptic symptoms. Consequently, clinical trials use different questionnaires which makes comparison of results difficult, and selection of one universal outcome measure seems favorable to uniformly assess FD symptoms in clinical trials.

In chapter $\mathbf{3}$ we describe the development of a novel ESM-based PROM. Use of a PROM based on the experience sampling technology (ESM) has advantages compared to standard retrospective (end-of-day) questionnaires. First, previous studies compared ESM-PROMs with retrospective (end-of-day) questionnaires and demonstrated over-reporting of symptoms by retrospective (end-of-day) 
questionnaires compared to ESM. More accurate reporting of symptoms by ESM might be due to the momentary assessment with a reduction of recall bias.,

Another major limitation of retrospective paper questionnaires is noncompliance. A previous study described an actual compliance rate of $10.9 \%$ with paper diaries although subjects reported a compliance rate of $90.5 \%$, which was however considered 'fake compliance'. In contrast, a compliance rate of $93.6 \%$ was found with use of an electronic diary. ${ }^{7}$ The experience sampling technology uses 10 random symptom assessments per day with the goal of completing at least six assessments per day. In a small trial with 26 IBS patients, the compliance rate with ESM was $76.8 \%$ (i.e. on $76.8 \%$ of the days at least 6 beeps were completed). ${ }^{6}$

Due to the repeated symptom assessment per day for several consecutive days, information is obtained about symptom variability with the potential to identify symptom triggers. Examples of potential triggers for dyspeptic symptoms are psychological aspects, contextual factors, nutrition and substance use. ${ }^{8,9}$ Identification of specific triggers per individual might guide therapy and contribute to a shift of a 'one-size fits all treatment approach' to a more 'individualized treatment approach' which might improve treatment outcome, avoid unnecessary and ineffective treatment strategies, and contribute to cost-effective health care.

Besides use of the ESM-based PROM for evaluation of treatment efficacy and identification of symptom triggers, it can also be used as a therapeutic application. Previous studies in patients with mental disorders described use of ESM as a method to provide personalized feedback on patterns of positive affect. Participants who received personalized feedback together with antidepressant medication demonstrated a greater decline in depressive symptoms compared to the control group who received antidepressant medication only. ${ }^{10}$ In addition, the experience sampling technology can also be used to implement psychotherapy in daily lives. ${ }^{11}$

In conclusion, use of an ESM-based PROM for symptom assessment in dyspeptic patients seems to have several important advantages compared to retrospective (end-of-day) questionnaires. Future studies are necessary to validate the novel ESMbased PROM before use in clinical trials.

\section{Part II Prediction of treatment outcome in upper gastrointestinal disorders}

\section{Achalasia}

Achalasia is an uncommon esophageal motility disorder with an estimated prevalence of 10 in $100.000 .^{12}$ Treatment of achalasia is focused on improving esophageal emptying by reduction of the relative obstruction at the gastroesophageal junction in order to improve symptoms and reduce the long-term risks of development of a megaesophagus and esophageal cancer. Short-term follow-up of patients after 
treatment is therefore recommended, although patient's symptoms are not a reliable predictor as symptom resolution might occur without improvement in esophageal emptying. ${ }^{13}$ As a consequence, functional testing is preferred but currently the optimal test has not been defined. In the ACG guideline two diagnostic modalities have been described: (1) timed barium esophagogram (TBE), and (2) esophageal manometry. ${ }^{12}$ Concordance between symptom reduction and improvement in esophageal emptying based on TBE has been found in $52-89 \%$ of patients. ${ }^{13-16}$ Due to the lack of concordance in the remaining patients, the ACG guideline recommends to repeat TBE annually to evaluate esophageal emptying. ${ }^{12}$ Important limitation for repetitive use of TBE is the radiation exposure. In addition, although several studies have shown that posttreatment LES pressure $<10 \mathrm{mmHg}$ was predictive of beneficial treatment outcome, a significant proportion of patients with persistent symptoms have a LES pressure below this cut-off value. ${ }^{12,14,15,17-19}$ Consequently, a novel diagnostic modality or parameter is warranted to evaluate treatment outcome and identify patients with need for retreatment. Based on previous studies which described impaired EGJ distensibility in (newly diagnosed) achalasia patients, we evaluated the potential of this novel diagnostic modality in evaluation of treatment outcome (chapter 4). ${ }^{20}$ In our small clinical trial, we were not able to differentiate between patients with good and poor clinical outcome based on EGJ distensibility, although conflicting results have been described by previous studies. ${ }^{15}$ These conflicting results together with the limited availability and expertise of this technique in medical centers, invasiveness of the EndoFLIP procedure, and high costs emphasize the need for additional research before use of this novel technique in routine clinical practice. Moreover, recently novel parameters of high-resolution (impedance) manometry have been proposed as factors predicting treatment outcome, and additional research is necessary to assess the potential for prediction of treatment outcome. Use of diagnostic parameters assessed during high-resolution (impedance) manometry has the benefit of a more worldwide available technology, without need for the purchase of a novel diagnostic modality.

In conclusion, there is need for a diagnostic modality or parameter to evaluate treatment outcome in achalasia patients in order to define the need for retreatment and reduce development of long-term complications. Additional research is necessary to assess the potential of the EndoFLIP technique and novel parameters measured with high-resolution (impedance) manometry.

\section{Gastroesophageal reflux disease}

The prevalence of gastroesophageal reflux disease (GERD) has increased over the last decades. The prevalence of at least weekly GERD symptoms is greatest in North America (19.8\%) and 15.2\% in Europe. ${ }^{21}$ In the late 1990's, GERD was found to be the gastrointestinal disorder with the highest annual direct costs in the Unites States of 
America accounting for $\$ 9.3$ billion, which increased to $\$ 12.1$ billion in 2014 . Majority of the direct costs were caused by pharmaceutical prescriptions ( $\$ 6-10$ billion), whereas hospital admissions, physician office visits and hospital outpatient visits made up the remainder of these costs. ${ }^{21}$ Despite the fact that the diagnosis of GERD can usually be made based on symptoms or an empiric trial of acid suppressive medication, $20-30 \%$ of 7 million upper gastrointestinal endoscopies performed annually in the USA are due to reflux symptoms. In addition to direct costs, GERD contributes also to indirect costs from missed work, diminished work productivity and impairment in performing daily activities. The estimated value of lost work productivity was $\$ 237$ per GERD patient over a 3-month period. ${ }^{21}$

The increasing prevalence of GERD together with the high socio-economic burden underline the need for effective treatment options. Although adequate symptom control is achieved with acid suppressive medication in the majority of patients, a subgroup of patients are considered non-responders, experience adverse effects from medical treatment or are not willing to take lifelong acid suppressive medication. Although (laparoscopic) fundoplication has been proven to be effective, it is accompanied by several perioperative and postoperative complications. One feared complication of antireflux surgery (ARS) is vagal nerve injury, which has been observed in up to $20 \%$ of patients undergoing ARS with a negative effect on long-term reflux control and a higher reoperation rate. ${ }^{22,23}$ Moreover, dysphagia might develop in case the surgical fundoplication led to a too tight wrap. The increasing prevalence and potential complications of traditional ARS, led to development of several minimally invasive treatment options including Transoral Incisionless Fundoplication (TIF). Although initial studies described promising short-term results, a recent systematic review and meta-analysis described superiority of laparoscopic Nissen fundoplication compared to TIF with regard to objective reflux control, increase in LES pressure and incidence of persistent esophagitis. ${ }^{24,25}$ The lack of durability seems an important limitation of TIF, and consequently members of a GERD expert panel were reluctant to advocate for TIF. ${ }^{26,27}$ It is however supposed that a selected subgroup of patients might benefit from TIF, which underlines the need for adequate patient selection. Based on previous studies, patients with no or small hiatal hernia $(\leq 2 \mathrm{~cm})$ and Hill grade I/II were considered best candidates for TIF. ${ }^{24,28}$ Both variables are however assessed by gastroenterologists during upper gastrointestinal endoscopy and are at risk for interrater variability. As the goal of ARS is to restore EGJ competence, we evaluated the effect of TIF on EGJ distensibility with the EndoFLIP technique (chapter 5 \& 6). Although preoperative EGJ distensibility was an independent predictor of objective treatment outcome after TIF, no difference in EGJ distensibility was found between patients with normalization of esophageal acid exposure time and patients with persistent abnormal acid exposure. Consequently, the role of the EndoFLIP technique in selection of patients for TIF seems limited at this moment. An attractive application of the EndoFLIP seems to be its intraoperative use to guide 
calibration of hiatal hernia repair and creation of the surgical fundoplication, although additional studies are necessary to define 'target values' for EGJ distensibility in order to improve adequate reflux control.

In conclusion, the increasing prevalence of GERD together with several (longterm) complications of traditional ARS led to development of minimally invasive (endoscopic) treatment options. Main limitation seems to be the limited durability with need for adequate patient selection. Additional research is necessary to identify variables for adequate patient selection.

\section{Gastroparesis}

Gastroparesis is a multifactorial disorder with incompletely understood pathophysiology. Treatment of patients with gastroparesis is challenging and treatment outcomes are often disappointing. Despite the fact that the ACG guideline discourages the use of botulinum toxin treatment in gastroparesis due to the lack of superiority compared to placebo in two clinical trials, it is still regularly used due to the lack of established alternatives. ${ }^{29-31}$ Botulinum toxin therapy is expensive with the need for repeated treatment procedures due to the temporary treatment effect. Previous studies described beneficial effects in a subgroup of patients, but identification of these patients is challenging. In chapter $\mathbf{7}$ we evaluated the potential roles of antroduodenal manometry and scintigraphy as predictors of treatment outcome. Variables of both diagnostic modalities were not able to predict treatment outcome and identify patients with potential beneficial treatment outcome. In addition, antroduodenal manometry is only available in a limited number of medical centers, and it is a cumbersome technique with regard to interpretation of results due to the absence of reference values, availability of different catheters and variations in study protocols. Besides the lack of a correlation with treatment outcome, the major limitation of scintigraphy is radiation exposure.

The lack of available diagnostic modalities to select patients for botulinum toxin treatment, together with the development of novel (endoscopic) treatment options targeted at the pylorus (e.g. gastric peroral pyloromyotomy) warrants the development of a novel technique focused on pyloric functioning. One modality of interest is the EndoFLIP technique as previous studies described impaired pyloric distensibility in a subgroup of gastroparesis patients. ${ }^{32}$ Identification of this subgroup might select patients for (endoscopic) pyloric treatment procedures in order to improve clinical outcome, and contribute to cost-effective health care.

In conclusion, identification of gastroparesis with impaired pyloric functioning seems desirable to select patients for (endoscopic) treatment procedures. The EndoFLIP technique seems of interest and additional research is necessary to define the exact role in patient selection. 


\section{References}

1. Mahadeva S, Goh KL. Epidemiology of functional dyspepsia: a global perspective. World J Gastroenterol. 2006;12(17):2661-6.

2. Sander GB, Mazzoleni LE, Francesconi CF, Balbinotto G, Mazzoleni F, Wortmann AC, et al. Influence of organic and functional dyspepsia on work productivity: the HEROES-DIP study. Value Health. 2011;14(5 Suppl 1):S126-9.

3. Ford AC, Forman D, Bailey AG, Cook MB, Axon AT, Moayyedi P. Who consults with dyspepsia? Results from a longitudinal 10-yr follow-up study. Am J Gastroenterol. 2007;102(5):957-65.

4. Ahlawat SK, Richard Locke G, Weaver AL, Farmer SA, Yawn BP, Talley NJ. Dyspepsia consulters and patterns of management: a population-based study. Aliment Pharmacol Ther. 2005;22(3):251-9.

5. Weinland SR, Morris CB, Hu Y, Leserman J, Bangdiwala SI, Drossman DA. Characterization of episodes of irritable bowel syndrome using ecological momentary assessment. Am J Gastroenterol. 2011;106(10):1813-20.

6. Mujagic Z, Leue C, Vork L, Lousberg R, Jonkers DM, Keszthelyi D, et al. The experience sampling method--a new digital tool for momentary symptom assessment in IBS: an exploratory study. Neurogastroenterol Motil. 2015;27(9):1295-302.

7. Stone AA, Shiffman S, Schwartz JE, Broderick JE, Hufford MR. Patient compliance with paper and electronic diaries. Control Clin Trials. 2003;24(2):182-99.

8. Feinle-Bisset C, Horowitz M. Dietary factors in functional dyspepsia. Neurogastroenterol Motil. 2006;18(8):608-18.

9. Mak AD, Wu JC, Chan Y, Chan FK, Sung JJ, Lee S. Dyspepsia is strongly associated with major depression and generalised anxiety disorder - a community study. Aliment Pharmacol Ther. 2012;36(8):800-10.

10. Kramer I, Simons CJ, Hartmann JA, Menne-Lothmann C, Viechtbauer W, Peeters F, et al. A therapeutic application of the experience sampling method in the treatment of depression: a randomized controlled trial. World Psychiatry. 2014;13(1):68-77.

11. Batink T, Bakker J, Vaessen T, Kasanova Z, Collip D, van Os J, et al. Acceptance and commitment therapy in daily life training: a feasibility study of an mHealth intervention. JMIR Mhealth Uhealth. 2016;4(3):e103.

12. Vaezi MF, Pandolfino JE, Vela MF. ACG clinical guideline: diagnosis and management of achalasia. Am J Gastroenterol. 2013;108(8):1238-49; quiz 50.

13. Vaezi MF, Baker ME, Richter JE. Assessment of esophageal emptying post-pneumatic dilation: use of the timed barium esophagram. Am J Gastroenterol. 1999;94(7):1802-7.

14. Rohof WO, Lei A, Boeckxstaens GE. Esophageal stasis on a timed barium esophagogram predicts recurrent symptoms in patients with long-standing achalasia. Am J Gastroenterol. 2013;108(1):49-55.

15. Rohof WO, Hirsch DP, Kessing BF, Boeckxstaens GE. Efficacy of treatment for patients with achalasia depends on the distensibility of the esophagogastric junction. Gastroenterology. 2012;143(2):328-35.

16. Vaezi MF, Baker ME, Achkar E, Richter JE. Timed barium oesophagram: better predictor of long term success after pneumatic dilation in achalasia than symptom assessment. Gut. 2002;50(6):765-70.

17. Eckardt VF, Aignherr C, Bernhard G. Predictors of outcome in patients with achalasia treated by pneumatic dilation. Gastroenterology. 1992;103(6):1732-8.

18. Eckardt VF, Gockel I, Bernhard G. Pneumatic dilation for achalasia: late results of a prospective follow up investigation. Gut. 2004;53(5):629-33.

19. Hulselmans M, Vanuytsel T, Degreef T, Sifrim D, Coosemans W, Lerut T, et al. Long-term outcome of pneumatic dilation in the treatment of achalasia. Clin Gastroenterol Hepatol. 2010;8(1):30-5.

20. Smeets FG, Masclee AA, Keszthelyi D, Tjwa ET, Conchillo JM. Esophagogastric junction distensibility in the management of achalasia patients: relation to treatment outcome. Neurogastroenterol Motil. 2015;27(10):1495-503.

21. Rubenstein JH, Chen JW. Epidemiology of gastroesophageal reflux disease. Gastroenterol Clin North Am. 2014;43(1):1-14. 
22. van Rijn S, Roebroek YG, Conchillo JM, Bouvy ND, Masclee AA. Effect of vagus nerve injury on the outcome of antireflux surgery: an extensive literature review. Dig Surg. 2016;33(3):230-9.

23. van Rijn S, Rinsma NF, van Herwaarden-Lindeboom MY, Ringers J, Gooszen HG, van Rijn PJ, et al. Effect of vagus nerve integrity on short and long-term efficacy of antireflux surgery. Am J Gastroenterol. 2016;111(4):508-15.

24. Cadiere GB, Buset M, Muls V, Rajan A, Rosch T, Eckardt AJ, et al. Antireflux transoral incisionless fundoplication using EsophyX: 12-month results of a prospective multicenter study. World J Surg. 2008;32(8):1676-88.

25. Richter JE, Kumar A, Lipka S, Miladinovic B, Velanovich V. Efficacy of laparoscopic nissen fundoplication vs transoral incisionless fundoplication or proton pump inhibitors in patients with gastroesophageal reflux disease: a systematic review and network meta-analysis. Gastroenterology. 2018;154(5):1298-308 e7.

26. Witteman BP, Conchillo JM, Rinsma NF, Betzel B, Peeters A, Koek GH, et al. Randomized controlled trial of transoral incisionless fundoplication vs. proton pump inhibitors for treatment of gastroesophageal reflux disease. Am J Gastroenterol. 2015;110(4):531-42.

27. Yadlapati R, Vaezi MF, Vela MF, Spechler SJ, Shaheen NJ, Richter J, et al. Management options for patients with GERD and persistent symptoms on proton pump inhibitors: recommendations from an expert panel. Am J Gastroenterol. 2018;113(7):980-6.

28. Testoni PA, Vailati C, Testoni S, Corsetti M. Transoral incisionless fundoplication (TIF 2.0) with EsophyX for gastroesophageal reflux disease: long-term results and findings affecting outcome. Surg Endosc. 2012;26(5):1425-35.

29. Camilleri M, Parkman HP, Shafi MA, Abell TL, Gerson L, American College of G. Clinical guideline: management of gastroparesis. Am J Gastroenterol. 2013;108(1):18-37; quiz 8.

30. Friedenberg FK, Palit A, Parkman HP, Hanlon A, Nelson DB. Botulinum toxin A for the treatment of delayed gastric emptying. Am J Gastroenterol. 2008;103(2):416-23.

31. Arts J, Holvoet L, Caenepeel P, Bisschops R, Sifrim D, Verbeke K, et al. Clinical trial: a randomizedcontrolled crossover study of intrapyloric injection of botulinum toxin in gastroparesis. Aliment Pharmacol Ther. 2007;26(9):1251-8.

32. Gourcerol G, Tissier F, Melchior C, Touchais JY, Huet E, Prevost G, et al. Impaired fasting pyloric compliance in gastroparesis and the therapeutic response to pyloric dilatation. Aliment Pharmacol Ther. 2015;41(4):360-7. 
Addendum 


\section{List of publications}

\section{Published articles}

Smeets F.G.M., Liedorp P.L., Van der Poel M., Miclea R., Masclee A.A.M., Pierik M. Anaplastic large cell T-cell lymphoma during ustekinumab treatment for Crohn's disease: a case report and review of the literature. Accepted Journal of Crohn's and Colitis 2019

Strijbos D., Keszthelyi D., Smeets F.G.M., Kruimel J.W., Gilissen L.P.L., De Ridder R., Conchillo J.M., Masclee A.A.M. Therapeutic strategies in gastroparesis: results of stepwise approach with diet and prokinetics, Gastric Rest and PEG-J. A retrospective analysis. Neurogastroenterol Motil 2019;31(6):e13588

Smeets F.G.M.* , Strijbos D.*, Keszthelyi D., Hoge C.V., Kruimel J.W., Conchillo J.M., Masclee A.A.M. Endoscopic Botulinum toxin for gastroparesis: results of a retrospective series. Gastrointest. Disord. 2019;1(1):164-75. (*Both authors contributed equally to the manuscript)

Smeets F.G.M., Keszthelyi D., Vork L., Tack J., Talley N.J., Simren M., Aziz Q., Ford A.C., Conchillo J.M., Kruimel J.W., Van Os J., Masclee A.A.M. Development of a real-time patient-reported outcome measure for symptom assessment in patients with functional dyspepsia using the Experience Sampling Method. Neurogastroenterol Motil. 2019;31(2):e13496

Smeets F.G.M., Masclee A.A.M., Conchillo J.M., Keszthelyi D. Systematic review: Disease-specific instruments to assess gastrointestinal symptoms in functional dyspepsia. Neurogastroenterol Motil. 2018 Mar 9 [Epub ahead of print]

Smeets F.G.M., Peters F.P.J. A male with intra-abdominal nodules on a CT scan. Ned Tijdschr Geneeskd. 2017;161:D1503

Smeets F.G.M., Masclee A.A.M., Keszthelyi D., Tjwa E.T., Conchillo J.M. Esophagogastric junction distensibility in the management of achalasia patients: relation to treatment outcome. Neurogastroenterol Motil. 2015; 27(10):1495-503

Smeets F.G.M., Masclee A.A.M., Keszthelyi D., Tjwa E.T., Conchillo J.M. Dilation in achalasia - is normal distensibility enough: Author's reply. Neurogastroenterol Motil. 2016; 28(1):154-5 
Smeets F.G.M., Keszthelyi D., Bouvy N.D., Masclee A.A.M., Conchillo J.M. Does measurement of esophagogastric junction distensibility by EndoFLIP predict therapyresponsiveness to endoluminal fundoplication in patients with gastroesophageal reflux disease? J Neurogastroenterol Motil. 2015;21(2):255-64

Smeets F.G.M., Keszthelyi D., Masclee A.A.M., Conchillo J.M. Measurement of esophagogastric junction distensibility may assist in selecting patients for endoluminal gastroesophageal reflux disease surgery: Author's reply. J Neurogasteroenterol Motil. 2015; 21(3):450-1

Smeets F.G.M.*, Aquarius M.*, Konijn H.W., Stassen P.M., Keulen E.T., Van Deursen C.T., Masclee A.A.M., Keulemans Y.C. Prospective multicenter validation of the Glasgow Blatchford bleeding score in the management of patients with upper gastrointestinal hemorrhage presenting at an emergency department. Eur J Gastroenterol Hepatol. 2015;27(9):1011-6 (*Both authors contributed equally to the manuscript)

Rinsma N.F., Smeets F.G.M., Bruls D.W., Kessing B.F., Bouvy N.D., Masclee A.A.M., Conchillo J.M. Effect of transoral incisionless fundoplication on reflux mechanisms. Surg Endosc. 2014;28(3):941-9 


\section{Dankwoord}

'Dankbaarheid is respecteren wat een ander je heeft gegeven'

Allereerst wil ik professor Bouvy bedanken, uw stimulans om tijdens de geneeskunde opleiding te starten met wetenschappelijk onderzoek op het gebied van de MDLheelkunde heeft de basis gecreëerd voor zowel dit proefschrift als mijn opleiding tot MDL-arts.

Uiteraard wil ik mijn promotieteam bedanken. Professor Masclee, dank voor uw vertrouwen, de begeleiding en de mogelijkheid om dit proefschrift te schrijven door het aangaan van een gecombineerd opleidings-onderzoekstraject. Dr. Conchillo, ik wil $\mathrm{u}$ bedanken voor het feit dat $\mathrm{u}$ me als student liet kennismaken met wetenschappelijk onderzoek en me wist te enthousiasmeren om te starten aan dit promotietraject. Daniel, je weet dat mijn dankbaarheid niet in een paar eenvoudige woorden valt uit te drukken. Je brede kennis, inspiratie, motivatie en de fijne, efficiënte samenwerking waren onmisbaar voor het voltooien van dit proefschrift.

Dank aan de leden van de beoordelingscommissie voor het lezen van dit proefschrift.

Tevens wil ik alle MDL collega's van het MUMC+ bedanken bestaande uit de MDLartsen, arts-assistenten, (arts-)onderzoekers, medewerkers van de endoscopie afdeling en functiekamer, evenals Mietsie, Elly en Nienke. Denise en Lisa, bedankt voor de prettige samenwerking.

Het Zuyderland Medisch Centrum wil ik danken voor het aangaan van het gecombineerde opleidings-onderzoekstraject en de bijbehorende financiering. Tevens wil ik dr. Looij als opleider, alle MDL-artsen, internisten, klinisch geriaters en artsassistenten hartelijk bedanken voor de fijne tijd tijdens mijn vooropleiding. Anouk, Paul, Joris en Ella, bedankt voor de gezellige momenten.

Uiteraard wil ik mijn vriendinnen Lisanne, Suzanne, Mirna, Petra, Maaike, Astrid en Maartje bedanken voor hun luisterend oor, geduld en fijne momenten van ontspanning.

Jean, Mariette, Esther en Rachel, ik ben uitermate dankbaar voor jullie warme vriendschap en steun, zowel tijdens de mooie als ook de moeilijke momenten van het leven.

Daniëlle, trots is denk ik het voornaamste woord. Trots ben ik op het feit je dat je mijn zusje bent. Uitermate trots ben ik op het feit dat je je hart hebt gevolgd, 
diergeneeskunde bent gaan studeren in het prachtige Utrecht en dit fantastisch hebt weten af te ronden. Bedankt voor het ontwerpen van de speciale kaft voor dit proefschrift.

Lieve mama en papa, uiteraard gaat mijn allergrootste dank uit naar jullie. Jullie hebben me onbezorgd laten opgroeien en hebben me alle mogelijkheden gegeven om me te ontwikkelen tot de persoon die ik ben geworden. Jullie geloof in mijn kunnen, jullie steun en onvoorwaardelijke liefde betekenen alles voor mij. Deze speciale dag had ik graag met zijn allen willen vieren, maar ik koester de mooie herinneringen.

Een hart dat klopt om leven en liefde te geven, Een hart om van te houden

Fabiënne 


\section{Curriculum vitae}

Fabiënne Geraldine Maria Smeets was born on October $20^{\text {th }} 1988$ in Brunssum, the Netherlands.

After graduation from the Sint-Janscollege in Hoensbroek in 2007, she started medical school at the Maastricht University, Faculty of Health, Medicine and Life Sciences. During medical school, she finished clinical and scientific traineeships at the division of Gastroenterology and Hepatology, Maastricht University Medical Center + $\left(\mathrm{MUMC}^{+}\right)$.

After obtaining her medical degree in 2013, Fabiënne started working as a PhD-fellow at the division of Gastroenterology and Hepatology of $\mathrm{MUMC}^{+}$under the supervision of prof. dr. A. Masclee, dr. J. Conchillo and dr. D. Keszthelyi. The research was performed within the School of Nutrition and Translational Research in Metabolism (NUTRIM) and partially funded by Zuyderland Medical Center (SittardGeleen).

In April 2015 she started her residency Gastroenterology and Hepatology under the supervision of prof. dr. A. Masclee. The first two years of her residency, she worked at the department of Internal Medicine at the Zuyderland Medical Center under the supervision of dr. B. Looij. Currently, Fabiënne is working at the division of Gastroenterology and Hepatology at the $\mathrm{MUMC}^{+}$under the supervision of prof. dr. A. Masclee. 
